\title{
Personalized computational modeling of vascular access creation
}

Citation for published version (APA):

Huberts, W. (2012). Personalized computational modeling of vascular access creation. [Doctoral Thesis, Maastricht University]. Maastricht University. https://doi.org/10.26481/dis.20120301wh

Document status and date:

Published: 01/01/2012

DOI:

10.26481/dis.20120301wh

Document Version:

Publisher's PDF, also known as Version of record

\section{Please check the document version of this publication:}

- A submitted manuscript is the version of the article upon submission and before peer-review. There can be important differences between the submitted version and the official published version of record.

People interested in the research are advised to contact the author for the final version of the publication, or visit the DOI to the publisher's website.

- The final author version and the galley proof are versions of the publication after peer review.

- The final published version features the final layout of the paper including the volume, issue and page numbers.

Link to publication

\footnotetext{
General rights rights.

- You may freely distribute the URL identifying the publication in the public portal. please follow below link for the End User Agreement:

www.umlib.nl/taverne-license

Take down policy

If you believe that this document breaches copyright please contact us at:

repository@maastrichtuniversity.nl

providing details and we will investigate your claim.
}

Copyright and moral rights for the publications made accessible in the public portal are retained by the authors and/or other copyright owners and it is a condition of accessing publications that users recognise and abide by the legal requirements associated with these

- Users may download and print one copy of any publication from the public portal for the purpose of private study or research.

- You may not further distribute the material or use it for any profit-making activity or commercial gain

If the publication is distributed under the terms of Article $25 \mathrm{fa}$ of the Dutch Copyright Act, indicated by the "Taverne" license above, 


\section{Personalized computational modeling of vascular access creation}

Wouter Huberts 
(c)Wouter Huberts, Maastricht 2012

All rights reserved. No part of this book may be reproduced, stored in a retrieval system, or transmitted, in any form or in any means, electronic, mechanical, photocopying, recording or otherwise, without the prior written permission from the author.

ISBN 978-90-8891-379-2

Cover design by Uco Egmond, Comic Design

Printed by Proefschriftmaken.nl || Printyourthesis.com 


\title{
Personalized computational modeling of vascular access creation
}

\author{
PROEFSCHRIFT \\ ter verkrijging van de graad van doctor \\ aan de Universiteit Maastricht, \\ op gezag van de Rector Magnificus, \\ Prof. mr. G.P.M.F. Mols, \\ volgens het besluit van het College van Decanen \\ in het openbaar te verdedigen \\ op donderdag 1 maart 2012 om 14.00 uur \\ door \\ Ir. Wouter Huberts \\ Geboren te Son en Breugel
}




\title{
Promotores
}

Prof.Dr.Ir. F.N. van de Vosse, Technische Universiteit Eindhoven Prof.Dr. T. Delhaas

\author{
Co-promotores \\ Dr.Ir. E.M.H. Bosboom \\ Dr. J.H.M. Tordoir
}

\section{Beoordelingscommissie}

Prof.Dr. G.W.H. Schurink (voorzitter)

Prof.Dr.Ir. E.J.E. Cottaar, SMPE/e, Technische Universiteit Eindhoven

Prof.Dr.Ir. A.P.G. Hoeks

Prof.Dr. K.M.L. Leunissen

Prof.Dr.Ir. P. Verdonck, IbiTech, Ghent University (Belgium) 


\section{Contents}

1 Background and aim of the thesis 1

1.1 Clinical background . . . . . . . . . . . . . . . 2

1.2 Computational modeling . . . . . . . . . . . . . 7

1.3 Aim and thesis outline . . . . . . . . . . . . . . 10

2 Lumped parameter segment $\quad 13$

2.1 Introduction . . . . . . . . . . . . . . . . . 15

2.2 Methods: Derivation of the lumped model . . . . . . . . . . . . . . 18

2.2.1 The approximate velocity profile function . . . . . . . . 18

2.2.2 Derivation of the lumped model . . . . . . . . . . . . . 19

2.3 Results: Comparison between the lumped model, boundary layer model and Womersley theory . . . . . . . . . . . . . . . . 22

2.3.1 Relative impedance ... . . . . . . . . . . . 22

2.3.2 Limiting cases . . . . . . . . . . . . . . . . 23

2.3.3 Simplified models ... . . . . . . . . . . 25

2.4 Discussion . . . . . . . . . . . . . . . . 26

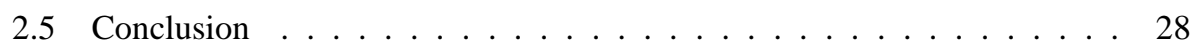

Appendix: The relative impedance for Womersley theory for small and large

Womersley parameters . . . . . . . . . . . . . . . 29

3 Pulse wave propagation model $\quad 31$

3.1 Introduction . . . . . . . . . . . . . . . . . 33

3.2 Material and methods . . . . . . . . . . . . . . . 37

3.2.1 The mathematical model . . . . . . . . . . . . . 37

3.2.2 Models for vascular access surgery . . . . . . . . . . . . 44

3.3 Results ......................... 52 
3.3.1 Vascular access planning ............... 52

3.4 Discussion . . . . . . . . . . . . . . . . . 57

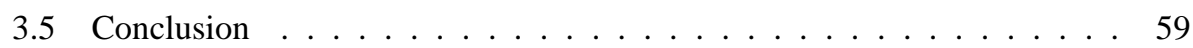

Appendix: Governing equations, electrical analog and numerical scheme . . . . 59

4 A sensitivity analysis to improve the measurement protocol 71

4.1 Introduction . . . . . . . . . . . . . . . . . 73

4.2 Material and methods . . . . . . . . . . . . . . . 75

4.2.1 The wave propagation model . . . . . . . . . . . 75

4.2.2 Variance-based method . . . . . . . . . . . . . . . 76

4.2.3 Global sensitivity analysis . . . . . . . . . . . . 79

4.3 Results ....................... 88

4.3.1 Mean brachial flow . . . . . . . . . . . . . . 92

4.3.2 Systolic pressure of the distal radial artery . . . . . . . . . . 96

4.4 Discussion . . . . . . . . . . . . . . . . . 100

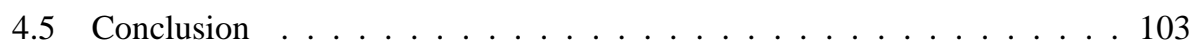

Appendix: Mathematical background and numerical implementation . . . . . 103

5 A sensitivity analysis to reduce the number of patient-specific inputs 113

5.1 Introduction . . . . . . . . . . . . . . . . . . 115

5.2 Material and methods . . . . . . . . . . . . . . . . 117

5.2.1 The wave propagation model . . . . . . . . . . . 117

5.2.2 Global sensitivity analysis . . . . . . . . . . . . . . 119

5.2.3 Input parameters and analysis . . . . . . . . . . . 121

5.3 Results . . . . . . . . . . . . . . . . . 126

5.4 Discussion . . . . . . . . . . . . . . . . . 132

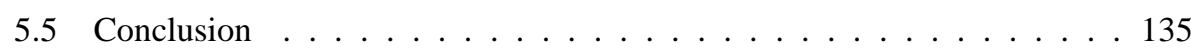

6 Model corroboration: An experimental study 137

6.1 Introduction . . . . . . . . . . . . . . . . . . . . . 139

6.2 Material and methods . . . . . . . . . . . . . . . . . 141

6.2.1 The mathematical model . . . . . . . . . . . . . . 141

6.2.2 The experimental model . . . . . . . . . . . . . . 144

6.2.3 Experimental corroboration: experiment versus pulse wave propagation model . . . . . . . . . . . . . . . . . 147

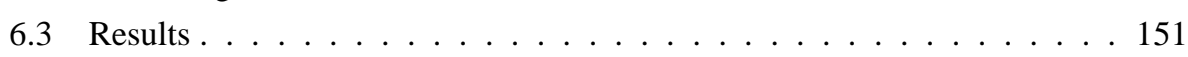

6.3 .1 Preoperative results . . . . . . . . . . . . 151 
6.3.2 Postoperative results . . . . . . . . . . . . . 151

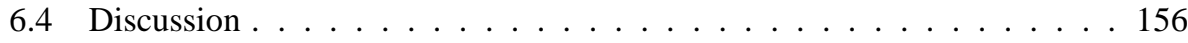

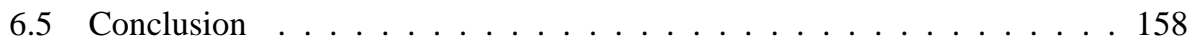

7 Model corroboration: A clinical feasibility study 161

7.1 Introduction . . . . . . . . . . . . . . . . . 163

7.2 Material and methods . . . . . . . . . . . . . . . . . . . 164

7.2.1 Study population . . . . . . . . . . . . . . . . . 164

7.2.2 Pulse wave propagation model . . . . . . . . . . . . . 164

7.2.3 Personalization of the pulse wave propagation model . . . . . . . 167

7.2 .4 Analysis . . . . . . . . . . . . . . . 168

7.3 Results . . . . . . . . . . . . . . . . . . 169

7.3.1 Vascular access configuration . . . . . . . . . . . . . 169

7.3.2 Absolute postoperative flow prediction . . . . . . . . . 170

7.3.3 Sensitivity analysis . . . . . . . . . . . . . 171

7.4 Discussion . . . . . . . . . . . . . . . . . . 172

Appendix: Uncertainty domains . . . . . . . . . . . . . . 175

8 General discussion $\quad 177$

8.1 Motivation, aim and methodology . . . . . . . . . . . . . . . . . . . . . . . 178

8.2 Computational model development . . . . . . . . . . . . . . . . 179

8.3 Model personalization . . . . . . . . . . . . . . 180

8.4 Model corroboration . . . . . . . . . . . . . . . . 181

8.5 Future perspectives . . . . . . . . . . . . . . . . 182

8.6 Concluding remarks . . . . . . . . . . . . . 185

$\begin{array}{lr}\text { Summary } & 199\end{array}$

$\begin{array}{lr}\text { Samenvatting } & 203\end{array}$

$\begin{array}{ll}\text { Dankwoord } & 207\end{array}$

$\begin{array}{lr}\text { Curriculum vitae } & 213\end{array}$

$\begin{array}{ll}\text { Scientific output } & 215\end{array}$ 
Contents 
Chapter 1

\section{Background and aim of the thesis}




\subsection{Clinical background}

\section{Kidney failure and hemodialysis}

The kidneys are organs in the human body that contribute to the maintenance of homeostasis, by means of regulation of water and electrolyte balance, and the excretion of metabolic waste products. The kidneys perform these functions by filtering the blood plasma and removing substances from the blood at a variable rate, depending on the needs of the body [1].

Patients suffering from end-stage renal disease (ESRD) have an irreversible decrease in kidney function to a level below $20 \%$ of normal kidney function [1]. Therefore, ESRDpatients need a renal replacement therapy. Due to a shortage of renal transplants, on January $1^{\text {st }} 2010$, 35\% of all 14690 ESRD-patients in the Netherlands were treated by hemodialysis [2]. In Europe, more than half-a-million ESRD-patients are dependent on hemodialysis therapy [3]; a number that is expected to increase annually by approximately $8 \%[4,5]$. During hemodialysis, the blood of the patient is withdrawn from the body using a pump with a flow rate of at least $300 \mathrm{ml} / \mathrm{min}$ and directed through an artificial kidney (dialyzer) where excess fluid, minerals and metabolic waste products are removed from the blood. A few centimeters upstream of the outlet, the cleaned blood is pumped back from the dialyzer into the body with the same flow rate (Figure 1.1). To enable hemodialysis a functioning vascular access is needed. The vascular access should deliver the high blood flow needed for the extracorporeal circulation and should have a caliber that enables repetitive cannulation over time to connect the patient with the dialyzer [6, 7].

\section{Vascular access}

Several types of vascular access are possible (Figure 1.2). A catheter inserted in one of the central veins (central venous catheter, $\mathrm{CVC}$ ) is the most simple option and enables immediate hemodialysis [7, 8, 9]. However, a CVC limits a patient in daily activities and is prone to thrombosis, infections, and to the development of central venous stenosis and obstructions $[7,8,9]$. Therefore, this type of vascular access is only used when acute hemodialysis is needed or when other types of vascular access are impossible [7, 8, 9]. Preferably, a vascular access is surgically created by permanently connecting an artery and a vein, either by using a graft (arteriovenous graft, AVG) or by directly connecting native vessels (arteriovenous fistula, AVF). In this way the peripheral resistance is bypassed, which results in a significant blood flow increase (five- to thirty fold), vessel dilatation and vessel wall remodeling. An arteriovenous fistula (AVF) is the primary choice because 


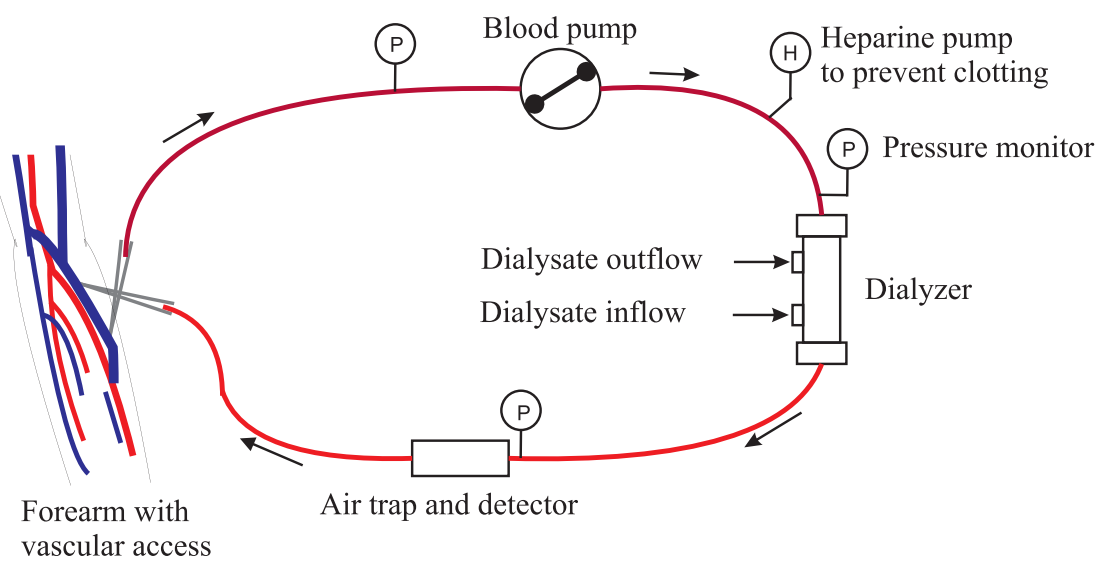

Figure 1.1: A schematic diagram of the hemodialyzer. The flow directions of the blood and dialysate fluid are indicated by arrows.

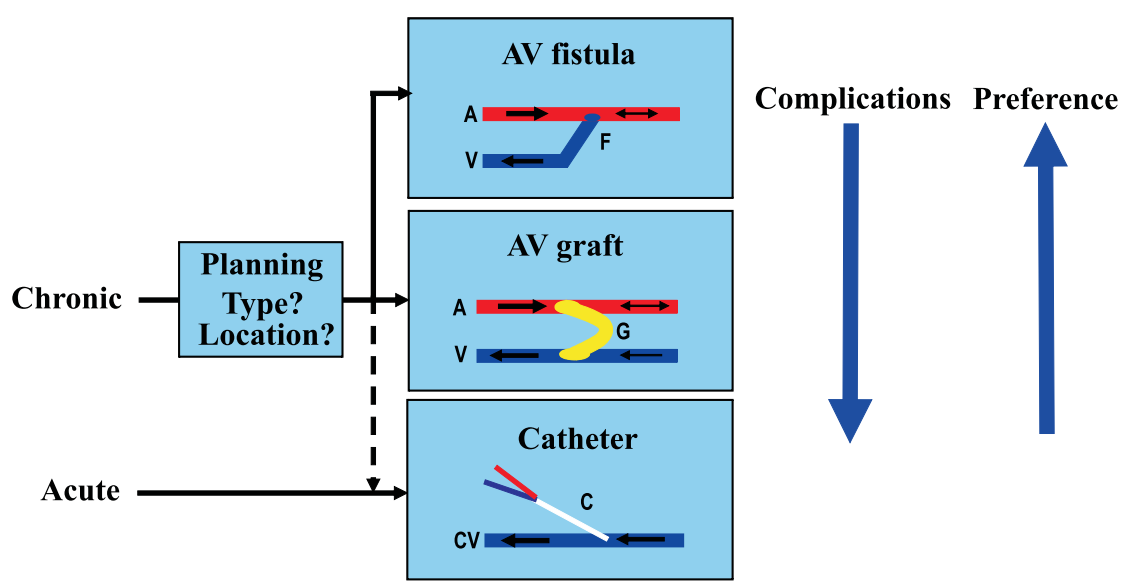

Figure 1.2: A schematic picture of the different access types. 
generally less interventions are required to preserve vascular access functioning $[8,9,10]$. An AVF is usually created in the arm. The exact site is a compromise between initial and long-term patency, complication rates and saving potential vascular access sites for future procedures $[11,12,13,14]$. For the former reasons, the radiocephalic AVF (RCAVF) that is created at wrist level by connecting the radial artery and the cephalic vein, is preferred over more proximal AVFs at elbow level, i.e. the brachiocephalic AVF (BCAVF) between the brachial artery and cephalic vein and the brachiobasilic AVF (BBAVF) between the brachial artery and the basilic vein (see Figure 1.3 for anatomical details).

\section{AVF complications}

In general, the upper arm AVFs lead to a larger postoperative flow than the lower arm AVFs. As a result, complications such as hand ischemia and cardiac failure occur more frequently in upper arm AVFs compared to lower arm AVFs. Of all upper arm AVFs 20\% is hampered by these complications $[7,8,12,13,14]$. Besides, in creation of a lower arm AVF the upper arm vessels are preserved in the event that a new vascular accesses is needed. On the other hand, especially RCAVFs are hampered by early failure due to thrombosis and non-maturation, which occurs in up to $50 \%$ of all cases $[6,7,10,12]$. In the case of non-maturation, the blood flow in the brachial artery (inflow) six weeks after surgery is smaller than $600 \mathrm{ml} / \mathrm{min}$, and/or the outflow vein is not dilated sufficiently $(<6$ $\mathrm{mm}$ ), and/or the vascular access is not located superficial enough (depth $>6 \mathrm{~mm}$ ), which all impede hemodialysis treatment $[6,7,8,9,10]$. Additionally to the aforementioned complications, AVF function is also interfered by long-term complications, like stenose development (60-100\% of all failed AVFs $[15,16,17])$ and thrombotic occlusions $(20 \%$ of all failed AVFs [17]), which are all likely to result from the dramatic change in hemodynamics after AVF creation [6, 7, 8, 9, 10]. All these complications result in additional interventions needed to maintain a vascular access suitable for hemodialysis [18]. It has been estimated that vascular access dysfunction is responsible for about $20 \%$ of all hospitalizations in the population of patients treated by hemodialysis [19]. 
Procedure for an upper arm AVF:

Torso and arm vasculature
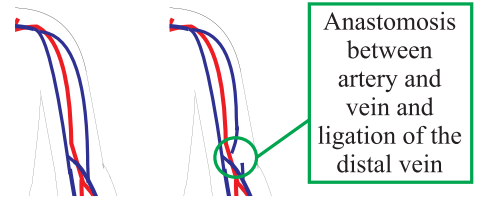

before surgery directly after surgery

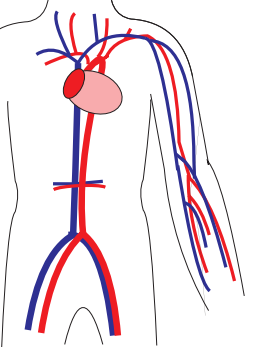

Procedure for a lower arm AVF:

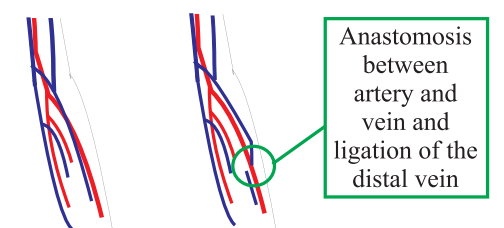

before surgery directly after surgery

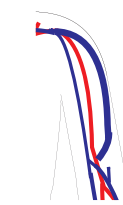

after six week (maturation)

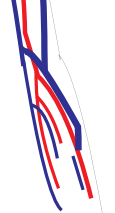

after six weeks

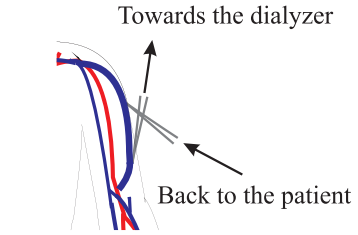

during hemodialysis session

Anastomosis:

Arterial inflow Venous outflow

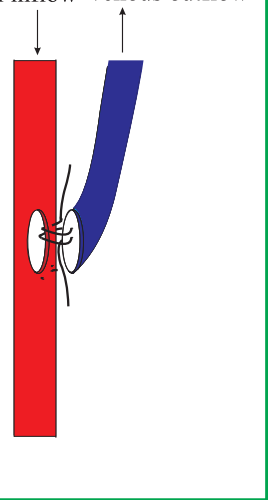

Figure 1.3: A schematic picture of the torso and arm vasculature including the major arteries and the superficial veins (left). The procedure of creation of an arteriovenous fistula (permanent connection between the artery and the vein) in the upper (middle, top) and lower (middle, bottom) arm and the connection of the VA to the dialyzer are depicted. At the right the anastomosis is shown in more detail. 


\section{Selecting the optimal AVF configuration}

The vascular surgeon tries to minimize the AVF associated complications by choosing the optimal AVF configuration for a specific patient. However, unless an extensive preoperative work-up, complications remain predominant and optimizing the AVF configuration is a difficult task. Postoperative flow enhancement differs between patients, because it is affected by multiple factors. Geometrical factors like vessel diameters and the location and the number of venous side-branches are patient-specific and play a role in postoperative hemodynamics $[15,20,21]$. In addition, the ability of the veins to adjust to increased pressure [16, 22] and the hyperaemic response of the peripheral bed in the hand resulting from fist clenching [20] are associated with AVF failure. Furthermore, a large intimamedia thickness of the radial artery is associated with early failure and too low vascular access flows [23, 24]. Moreover, the presence or occurrence of kinks, vessel twists and stenoses will impede blood flow since these geometries will induce additional resistance against flow $[25,26,27,28,29]$. An extra difficulty is formed by the anastomosis; the surgeon can control the size of the arteriotomy (longitudinal incision in the artery) only to some extend, because the surgical procedure is restricted by the anatomical location and size of the vessels involved and their surrounding tissues [30].

In clinical practice, the surgeon aims to collect as much information as possible regarding these influential factors before he selects the optimal site for AVF creation. An extensive preoperative vessel assessment is used in clinical practice to determine the continuity and caliber of the vessels. Arterial pulsatility and the continuity of both arteries and veins are assessed by palpation. Additionally, arterial and venous diameters, the brachial inflow, the caliber of venous side-branches and the occurrence of possible stenosis are determined by duplex ultrasound measurements [7, 8, 31]. Despite this approach, complications as nonmaturation, cardiac failure and hand ischemia still occur.

From literature, it is known that a too low brachial artery flow measured directly after surgery is indicative for non-maturation [10, 32], while a high postoperative brachial artery flow ( $>30 \%$ of the cardiac output) increases the risk on cardiac failure and hand ischemia $[12,13,14]$. In addition, the systolic finger pressure distal to the anastomosis is a clinical indicator for hand ischemia $[7,8,9]$. Hence, a tool that preoperatively predicts postoperative hemodynamics and more specifically immediate postoperative brachial artery flow and systolic finger pressure, can support the selection of the AVF configuration. A computational model fed by patient-specific data that simulates hemodynamics in a vascular network could serve as such a tool. An advantage of a computational model is that the different factors that influence the success of AVF creation can easily be va- 
ried and examined, because it relates these factors by means of physical laws. Moreover, the computational model can be used to examine the effect of different AVF configurations before surgery. As a result, the surgeon obtains extra information that can help in decision-making of AVF surgery.

\subsection{Computational modeling}

In cardiovascular research, computational modeling has extensively been used for different purposes. Models have been used to get insight into physiological $[33,34,35,36]$ and pathophysiological phenomena [25, 37, 38, 39], for the design and evaluation of medical devices [40, 41, 42] and to develop training simulators for medical interventions [43, 44]. In addition, computational models have been used to determine parameters that are not directly accessible by measurements $[45,46,47]$ or to assist physicians by making a diagnosis $[48,49]$. Finally, some previous studies have described the use of computational models to predict hemodynamic changes after creation of femoro-popliteal [50], thoracothoraco aortic [51, 52] and aorto-femoral bypassess [52]. However, the use of predictive models is not common in clinical practice yet.

\section{Mathematical models}

The type and complexity of the applied models depend on the objective of the study. Three-dimensional models (3D) $[38,53,54]$ are used to study local hemodynamics (e.g. velocity fields, wall shear stresses, local pressures and flows). Disadvantages of 3D models are the difficulties in defining proper patient-specific boundary conditions and, moreover, the long CPU times for 3D model calculations. For these reasons, 3D models are less suitable to calculate pressure, flow and wall shear stress distributions over the complete vascular system $[51,55]$.

The most simple models that are used to investigate the whole cardiovascular system are two-, three- or four-element windkessel models in which arterial wall and blood properties are represented by electrical elements, e.g. the aortic compliance with a capacitor, blood inertia with an inductor and peripheral resistance with a resistor [47, 56, 57]. More detailed lumped parameters models, consisting of more electrical elements, have also been used [34, 35, 58]. However, lumped parameter models fail to explain phenomena of pulse wave propagation through the arterial tree because the spatial information of the distributed arterial properties is lost. For an analysis of pressure and flow wave propagation, a model is required which includes the multi-branched configuration of the arterial system 
and a description of the distributed nature of arterial properties. For this purpose, pulse wave propagation models (cited below and for a detailed review we refer to [55]) are used in which the vascular system is divided into segments that represent local blood and vessel wall properties. All segments are serially connected based on the anatomical configuration. For each segment, the relation between blood pressure and flow is described by the one-dimensional momentum and continuity equations derived by Hughes and Lubliner [59] and presented in the appendix of Chapter 3. In the momentum equation, approximations for the wall shear stress and the convective acceleration term are needed. These can be estimated from a large variety of velocity profiles based on Poiseuille [51, 52], Witzig-Womersley theory for pulsatile flow [60], the Young and Tsai formulation [25], and approximate velocity profiles $[61,62]$. In addition, a constitutive law is used to describe vessel wall behavior. The resulting equations can be solved for pressure and flow by several numerical techniques like the method of characteristics [33, 60], the spectral element method [61, 63] and the finite element method [51, 52]. These types of models is here referred to as one-dimensional (1D) pulse wave propagation models. Pulse wave propagation models in which segments are lumped (spatial information within each segment is lost) into an electrical analog are also used [36, 40, 64] and here referred to as distributed lumped parameter pulse wave propagation models.

\section{Predictive modeling}

For predictive vascular surgery pulse wave propagation models are most appropriate because they have lower computational costs than 3D models and because they contain more spatial information than lumped parameter models. The spatial information is essential in planning vascular surgery. In pulse wave propagation models nonlinear equations can be easily incorporated, either to describe the nonlinear relationship between cross-sectional area and pressure $[61,65]$, or to capture additional pressure drops resulting from stenoses, kinks or anastomoses [50, 51]. For vascular access surgery correct modeling of the anastomosis is crucial.

Up till now, pulse wave propagation models have mostly been used to describe the hemodynamics. When using these models for predictive simulations, they should be fed by patient-specific data. Hence, based on patient-specific measurements or estimations from literature, numerical values, which are hampered by uncertainty, are assigned to the model input parameters. In this thesis, model input parameters are defined as given in Figure 1.4. The need for patient-specific model input parameters implies that a balance needs to be found for the complexity of the model. The complexity of the model should be sufficient 


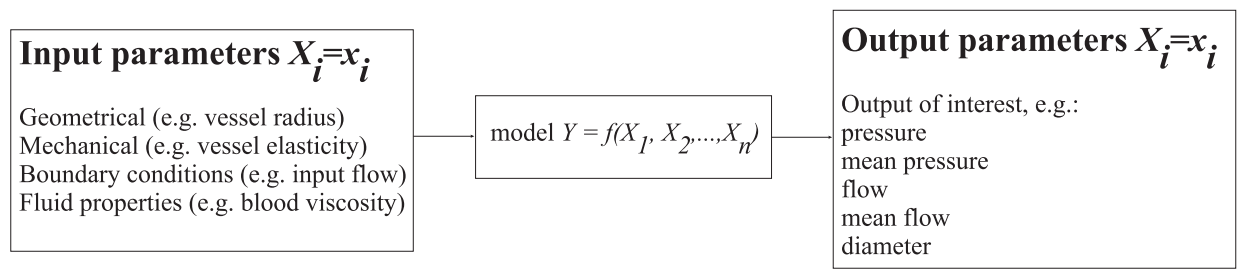

Figure 1.4: The definition of input and output parameters that is used in this thesis. The lower case $x_{i}$ indicates that a numerical value is assigned to the model input parameter $X_{i}$.

to describe the hemodynamics and physiology of interest. However, increasing model complexity will, in general, also increase the number of model input parameters. In practice, measuring all input parameters is impossible, and, in addition, measurements are hampered by inaccuracies. Model input uncertainty will result in uncertainties in model output (Figure 1.5). Thus, to obtain a prediction with minimal uncertainty in the output an optimum needs to be found between model complexity and the number of model input parameters (Figure 1.5)[66, 67].

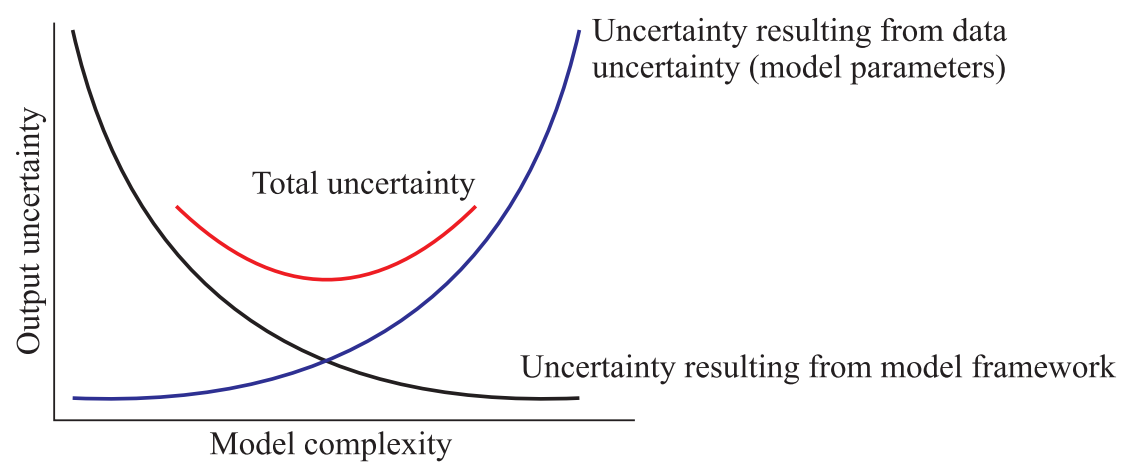

Figure 1.5: The contents of the uncertainty of predictions with personalized models is shown as function of model complexity. Based on [66, 67].

Nevertheless, in personalization of the model, not all model parameters are as influential on the output. The influence of a parameter on the output of the model can be derived 
from a sensitivity analysis $[68,69]$. A sensitivity analysis is applied to investigate how the uncertainty in the output from a computational model can be apportioned, qualitatively or quantitatively, to the different uncertainties in the input parameters, whereas an uncertainty analysis determines the output uncertainty $[68,69]$. A sensitivity analysis can either be local or global. In a local sensitivity analysis the change in output $\mathrm{Y}$ is determined after a change in input $X_{i}$ around its reference value $X_{i, 0}$, while all other input parameters are fixed (the one-factor-at-a-time approach). As a result, the complete input parameter space is only locally explored. This approach fails when interactions between input parameters are present. In that case, a global sensitivity analysis should be used in which the complete input parameter space is explored [68, 69]. In the field of cardiovascular research, sensitivity analyses on computational models have previously been performed [39, 70, 71, 72, 73]. However, the number of studies including a sensitivity analysis is relatively small compared to the number of models used in cardiovascular research. This is remarkable, especially in patient-specific modeling, since a proper sensitivity analysis is generally considered to be essential before inferences with the model can be made $[68,69]$. In the field of econometrics and environmental sciences, where predictive models are used to make inferences, variance-based global sensitivity analyses are extensively used and considered to be the current best available practice [68, 69, 74, 75]. With this method, model parameters can be identified that result in the largest reduction in output variance when measured accurately (parameter prioritization). Moreover, the method can be used to determine model parameters that can be fixed and made into a constant (parameter fixing) because they do not significantly influence the output. For our application in AVF surgery such an approach can be very useful to obtain insight in how to optimize model personalization.

\subsection{Aim and thesis outline}

In this study, we aim to develop a pulse wave propagation model that is able to predict hemodynamics after AVF creation. In addition, its applicability to support clinicaldecision making in vascular access surgery is examined. For this, we propose a multi-step approach (Figure 1.6) in which the model will be derived, personalized and corroborated. Model corroboration is here defined as quantitative and qualitative validation. 


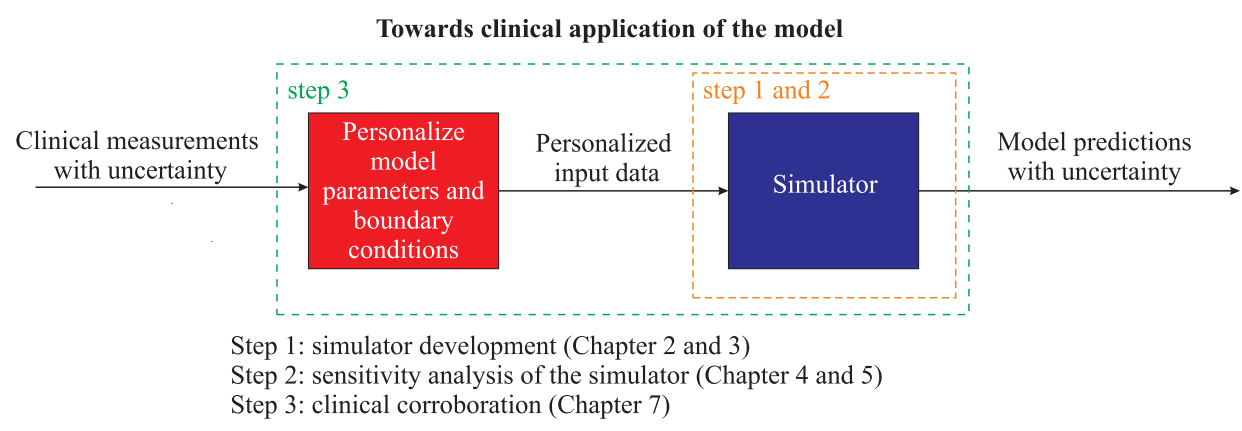

Figure 1.6: The steps that we propose to do towards clinical application of the model are shown schematically.

First, a pulse wave propagation model (simulator) will be developed (Chapter 2 and 3) that is able to predict pressure and flow waveforms in the arm vessels after the creation of different AVF configurations (i.e. RC-, BC- and BBAVF). The pulse wave propagation model should include the major arteries (inflow) and veins (outflow) of the AVF tract and the anastomosis region. The model's implementation should be robust with regard to the model input parameters to deal with a large variety in inputs and imperfect datasets common in clinical practice.

As second step, a global variance-based sensitivity analysis will be performed to determine which parameters are most important to measure accurately since they result in the largest reduction in output uncertainty (Chapter 4). Furthermore, model parameters will be indicated that can be set to a generic population value and for which no measurements are required (Chapter 5). The insight obtained in this second step will be used to personalize the computational model in the third step. Moreover, an uncertainty analysis will be performed to determine the propagation of model input uncertainties to the output uncertainty (precision of the predictions).

In the third step, the pulse wave propagation model will firstly be corroborated with a oneto-one silicone network model of the arm vasculature built in a mock loop in Chapter 6 $[76,77]$. The advantage of an experimental corroboration is that model parameters can be determined more easily and with higher precision than in patients. In addition, it is easier to accurately measure pressure and waveforms on several locations. Comparison of this experimental data to model simulations can give more insight into the correct description of pressure and flow wave propagation. Thereafter, personalized model predictions with their precision will be used to clinically corroborate the pulse wave propagation model 
(Chapter 7). For this, the flow predictions are used to select the best AVF configuration. The suggestion of the model will be compared to the choice of an experienced surgeon. Furthermore, the predicted postoperative flow will be compared to the actual flow one week after surgery to determine the accuracy of the model.

In Chapter $\mathbf{8}$ the major findings will be discussed and the results will be put into a broader perspective. 


\section{Chapter 2}

\section{Lumped parameter segment}

This Chapter is based on:

W. Huberts, E.M.H. Bosboom and F.N. van de Vosse, A lumped model for blood flow and pressure in the systemic arteries based on an approximate velocity profile function, Math. Biosc. Eng., 6(1), 27-40, (2009) 


\begin{abstract}
Previously, by assuming a viscous dominated flow in the boundary layer and an inertia dominated flow in the vessel core, a velocity profile function for a $1 \mathrm{D}$-wave propagation model was derived. Because the time dependent shape of the velocity profile in this boundary layer model depends on the size of the inviscid core and the boundary layer, and thus on the Womersley number, it differs along the arterial tree. In this study we evaluated a lumped model for a vessel segment in which the element configuration is based on physical phenomena described by the boundary layer model and for which all parameters have a physically based quantitative value dependent on the Womersley number. The proposed electrical analog consists of a Womersley number dependent resistor and an inductor arranged in parallel, representing the flow impedance in respectively the vessel core and the boundary layer, in series with a second resistor. After incorporating a capacitor representing the vessel compliance in this rigid tube model, the element configuration resembles the configuration of the four-element windkessel model. For arbitrary Womersley numbers the relative impedance of Womersley theory is approximated with high accuracy. In the limits for small and large Womersley numbers the relative impedances of the proposed lumped model correspond exactly to Womersley theory.
\end{abstract}




\subsection{Introduction}

Windkessel $[47,56,57,78]$ as well as lumped parameter models $[34,35,36,58,64,79$, $80,81]$ are used to simulate pressure and blood flow in the arterial system. The first windkessel model was introduced by Frank et al. [56]. This model consists of a capacitor representing the aortic compliance and a constant resistor representing the peripheral resistance. Westerhof et al. [57] extended this model with an extra resistor, which is thought to be the aortic characteristic impedance. As a result, a better representation of the medium- to high-frequency behavior of the systemic input impedance is obtained. Stergiopulos et al. [47] concluded that the four-element Windkessel model with an inertial term in parallel with the characteristic impedance, is even superior to the three-element Windkessel model in describing the behavior of the entire systemic tree or as a model for parameter estimation of vascular properties. Stergiopulos et al. concluded that the inertial term represents the total inertia of the blood. In addition, they stated that for the aorta the configuration of an inertial term in parallel with a characteristic impedance seems correct because for high frequencies the aorta behaves like a reflectionless tube and for low frequencies the local properties of the aorta (i.e., the characteristic impedance) are negligible. However, a quantitative physical origin for a parallel configuration was not given. Windkessel models fail to explain the phenomena of pulse wave propagation throughout the arterial tree, as the inherent property of the windkessel model assumes an infinite wave velocity. To study the wave phenomenon, therefore, arterial tree models based on transmission line theory have been developed [36, 82]. For these models the vascular system is divided (lumped) into segments that represent the local blood (density $\rho$, kinematic viscosity $\nu$ ) and vessel wall (radius $a$, compliance $C_{v}$ ) properties. All segments are connected based on the anatomical configuration to obtain a transmission line. These lumped parameter pulse wave propagation models include the multi-branched configuration of the arterial system and a description of the distributed nature of arterial properties. In Noordergraaf's model [82] a passive electrical analog was chosen, which was based on a comparison between equations describing propagation along a transmission line (the two telegraph equations) on the one hand and a simplified equation of motion of the blood and the equation of continuity for fluid flow in a short arterial segment on the other hand. The lumped segments consisted of an inductor $L$ in series with a resistor $R$ that represent respectively the blood inertia and the viscous blood flow resistance (Figure 2.1). In addition, the compliance $C$ of the vessel wall was modeled with a capacitor.

In large arteries, like the aorta, the wave phenomena are inertia dominated implying that the non-stationary acceleration term in the $1 \mathrm{D}$ momentum equation is dominant over the 
viscous term, i.e. the Womersley number $\alpha=a \sqrt{\frac{\omega}{\nu}} \gg 1$ with angular frequency $\omega$. A good approximation of the parameters of the lumped model (values for $R$ and $L$ ) can be derived from the vessel geometry and mechanical properties using a flat velocity profile. In small vessels $(\alpha \ll 1)$, i.e. arteries with a diameter smaller than $2 \mathrm{~mm}$, viscous forces are dominant and approximate values for $R$ and $L$ can be derived using a quasi-static Poiseuille profile. In Noordergraaf's model a flat velocity profile is assumed in the calculation for the inertial term whereas a parabolic velocity profile is assumed for the viscous term, so the two limiting cases were combined in each of the single vessels independent of the vessel size and frequency of the pressure and flow pulsations. Because of the pulsatile nature of the blood flow a phase difference will be present between the velocity in the core of the vessel and the velocity in the boundary layer close to the vessel wall. However, this will lead to frequency dependent values for the inductor $L$ and resistor $R$. To take the balance between viscous and inertial forces into account, Jager et al. derived an extended electrical network [64], that consisted of several frequency independent inductors and resistors for every segment (Figure 2.1). At higher Womersley numbers, a higher number of extra resistors and inductors were needed to accurately describe the longitudinal impedance [64]. Given the radii of the arteries and the harmonic that contains most wave energy, it is possible to derive an electrical transmission line of the total arterial tree that incorporates the balance between inertia and viscous forces. Such a model was made by Westerhof et al. [36], however, the network configuration is complex and totally based on a mathematical derivation in which the physical origin of the pressure and flow waves is not apparent.

Olufsen et al. [83] derived lumped models starting with the one-dimensional axisymmetric Navier-Stokes equation for time-dependent blood flow in rigid tubes and by applying Laplace transformation and inversion via residue theory. For tubes with a radius between 5 and $15 \mathrm{~mm}$ (i.e. Womersley numbers between approximately 5 and 15) Olufsen et al. found a lumped model which has the same configuration as the four-element windkessel model. This model configuration is less complex than Jager's network. However, the values of the resistor and inductor did not depend on the Womersley number, but were assumed to be constant for a range of radii.

In this study, we aim to derive a lumped model for a vessel in which the element configuration is based on physical phenomena and for which all parameters have a physically based quantitative value dependent on the Womersley number. In the limiting cases for small and large Womersley numbers, the impedance of the derived lumped model should correspond to Womersley theory. 


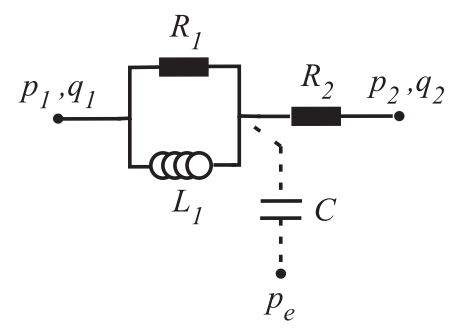

(a)

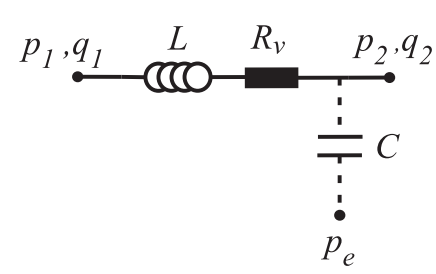

(b)

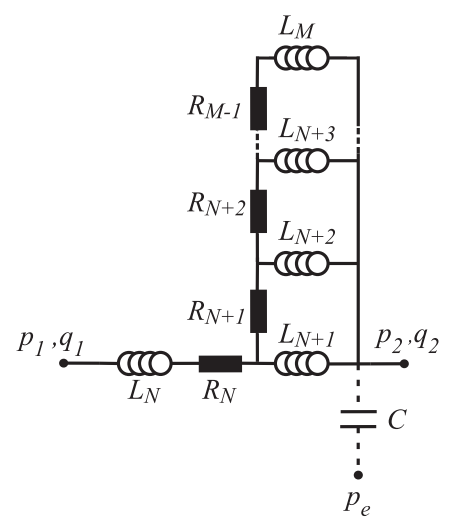

(c)

Figure 2.1: The electrical analog of the longitudinal impedance of (a) the proposed electrical analog based on the boundary layer model, (b) Noordergraaf's model and (c) Jager's model. The capacitor $C$ can be added to obtain a compliant tube model.

We based this study on a wave propagation model with a time and frequency dependent approximate velocity profile function derived by Bessems et al. [61]. They developed a one-dimensional model of blood flow in arteries without a priori assuming a shape for the velocity profile. The resulting approximate velocity profile consists of an inertia dominated flow in the core of the vessel and a viscous dominated flow near the vessel wall. The size of the inviscid core and the boundary layer depend on the Womersley number. Therefore in this model the various velocity profile shapes along the arterial tree differ and form a good approximation of the velocity profiles obtained from Womersley theory [84] with respect to the nonlinear term $\frac{\partial}{\partial z}\left(\int v_{z}^{2} d \Omega\right)$ and the friction term $\tau_{w}=\eta \int \frac{\partial v_{z}}{\partial r} d \Gamma$ [61] in the 1D momentum equation derived by Hughes and Lubliner [59] and presented in Chapter 3. 
To capture this in an electrical analog, intuitively, an inductor in parallel with a resistor is expected, representing the flow impedance in respectively the core of the vessel and near the vessel wall. Because for steady flow the total vessel impedance in the electrical analog should converge to a Poiseuille resistance, a Poiseuille resistor is added in series with the parallel arrangement of the inductor and resistor. In this paper, in Section 2, the approximate velocity profile function given by Bessems et al. is briefly described. In addition, mathematical expressions for the parameters in the proposed lumped model are derived. Thereafter, in Section 3, the relative impedance of the proposed lumped model normalized to the Poiseuille resistance is compared with the relative impedance derived from the approximate velocity profile function to motivate our choices. Next, the relative impedance is compared with Womersley theory to show the difference between Womersley theory and our model. In addition, the limiting cases for the relative impedances of the proposed lumped model and Womersley theory are compared. This is done for large $(\alpha \gg 1)$ and small $(\alpha \ll 1)$ Womersley numbers, representing respectively a flat and a parabolic velocity profile. Furthermore, simplified models are derived in case of Womersley numbers $\alpha \leq \sqrt{2}$ and $\alpha>\sqrt{2}$. These $\sqrt{2}$-limits stem from the derivation of the approximate velocity profile. Finally, the proposed lumped model is discussed in Section 4 and our findings are compared with literature.

\subsection{Methods: Derivation of the lumped model}

The lumped model proposed in this article is based on the wave propagation model with an approximate velocity profile function that was derived by Bessems et al. [61] and is further referred to as boundary layer model. In the first subsection the approximate velocity profile function will be described shortly. For a detailed derivation we refer to Bessems et al. [61]. An experimental validation of the model can be found in Bessems et al. [63]. The derivation of our electrical analog is described in the second subsection.

\subsubsection{The approximate velocity profile function}

To derive the approximate velocity profile function, Bessems et al. [61] considered the Navier-Stokes equation for fully developed flow in straight tubes driven by a given pressure gradient:

$$
\rho \frac{\partial v_{z}}{\partial t}=-\frac{\partial p}{\partial z}+\eta \frac{1}{r} \frac{\partial}{\partial r}\left(r \frac{\partial v_{z}}{\partial r}\right)
$$


in which $v_{z}$ is the axial velocity, $\frac{\partial p}{\partial z}$ the pressure gradient, $\rho$ the blood density, $\eta$ the dynamic viscosity and $r$ the radial coordinate.

In the boundary layer close to the vessel wall the viscous forces are dominant whereas in the center of the vessel the inertial forces are dominant. Between the viscous boundary layer and the vessel core there is then a transition layer in which a balance between viscous and inertial forces exists, as described by (2.1). Bessems et al. [61] assume the transition layer to become infinitely small. In addition, it is assumed that there is a balance between the inertial forces and the viscous forces at the transition from the boundary layer to the central core and that at the transition in the axial direction the velocity in the boundary layer equals the velocity in the central core. Under these assumptions it can be derived that the relation between the size of the central core and the Womersley number $\alpha$ can be given by:

$$
\frac{a_{c}}{a}=\max \left[0,1-\frac{\sqrt{2}}{\alpha}\right]
$$

in which $a_{c}(\alpha)$ is the Womersley number dependent radius of the central core and $a$ is the vessel radius. The velocity profile function given by Bessems et al. is related to $\zeta_{c}=\left(\frac{a_{c}}{a}\right)^{2}$ according to the following equation:

$$
v_{z}=-\frac{\ln \hat{\zeta}}{1-\zeta_{c}} \frac{q}{A}-\frac{a^{2}}{4 \eta}\left[1-\hat{\zeta}+\frac{1}{2}\left(\zeta_{c}+1\right) \ln \hat{\zeta}\right] \frac{\partial p}{\partial z}
$$

in which $q$ is the flow, $A$ the cross-sectional area, and $\hat{\zeta}=\max \left[\left(\frac{r}{a}\right)^{2}, \zeta_{c}\right]$. The shapes of the velocity profiles along the arterial tree computed with this boundary layer model are a good approximation of the velocity profiles obtained from Womersley theory with respect to the nonlinear term $\int v_{z}^{2} d \Omega$ and the friction term $\eta \int \frac{\partial v_{z}}{\partial r} d \Gamma[61]$. The advantage of the boundary layer model is that it can be applied in the time domain.

\subsubsection{Derivation of the lumped model}

To derive a lumped model we use the same assumptions as Bessems et al. in the derivation of its approximate velocity profile function. Intuitively, the lumped model consists then of a parallel arrangement of a resistor per segment length $R_{1}$ and an inertance per segment length $L_{1}$ that represent respectively the viscous resistance in the boundary layer and the inertia dominated impedance in the central core. To let the electrical model converge to a Poiseuille resistance for steady flow a second resistor $R_{2}$ is introduced in series with the parallelly arranged $R_{1}$ and $L_{1}$ (Figure 2.1). The derivation is restricted to rigid tubes, 
but can easily be extended with a capacitor to model the storage capacity of the vessel without changing the derived model parameters.

In this subsection the derivation of mathematical expressions for the resistors $R_{1}$ and for the inertance $L_{1}$ from the boundary layer model proposed by Bessems et al. [61] is described. A mathematical expression for the resistor $R_{2}$ follows directly from the definition of a Poiseuille resistance. The other two parameters are derived by comparing the longitudinal impedance of the proposed electrical analog with the longitudinal impedance in the boundary layer model. The longitudinal impedances are complex numbers and because the longitudinal impedances of both models should be the same, the real and imaginary parts are compared separately. In this way two equations with two unknowns $\left(R_{1}\right.$ and $\left.L_{1}\right)$ are obtained. From those two equations, the mathematical expressions for $R_{1}$ and $L_{1}$ are derived.

In Bessems' boundary layer model, by neglecting the external forces - the nonlinear term and the diffusion term, the 1D-momentum equation for flow through a rigid vessel is given by [61]:

$$
-\frac{\partial p}{\partial z}=\left[\frac{c_{q}}{2-c_{p}}\right] R_{v} q+\left[\frac{1}{2-c_{p}}\right] L \frac{\partial q}{\partial t}
$$

in which $p$ is the pressure, $z$ is the longitudinal direction, $R_{v}$ is the Poiseuille resistance per segment length $\left(\frac{8 \eta}{\pi a^{4}}\right)$, and $L$ is the inertia per segment length $\left(\frac{\rho}{\pi a^{2}}\right)$. The functions $c_{p}$ and $c_{q}$ are dependent on the squared radius of the vessel core $\zeta_{c}$ and thus on the Womersley number $\alpha$ according to [61]:

$$
c_{p}=1+\frac{1}{2}\left(1-\zeta_{c}\right) \quad \text { and } \quad c_{q}=\frac{1}{2}\left(1-\zeta_{c}\right)^{-1}
$$

For Womersley numbers smaller than $\alpha \leq \sqrt{2}$ the central core disappears, see (2.2), resulting in Poiseuille flow. By using (2.5), $c_{p}$ and $c_{q}$ for $\alpha \leq \sqrt{2}$ are given by:

$$
c_{p}=3 / 2, \quad c_{q}=1 / 2
$$

For large Womersley numbers $(\alpha>\sqrt{2}) c_{p}$ and $c_{q}$ depend on the core radius. After substitution of (2.2) in (2.5) $c_{p}$ and $c_{q}$ are given by [61]:

$$
c_{p}=1+\frac{\sqrt{2}}{\alpha}\left(1-\frac{\sqrt{2}}{2 \alpha}\right), \quad c_{q}=\frac{\alpha}{4 \sqrt{2}}\left(1-\frac{\sqrt{2}}{2 \alpha}\right)^{-1} \text {. }
$$


By introducing the harmonics

$$
\frac{\partial p}{\partial z}=\frac{\partial \hat{p}}{\partial z} e^{j \omega t}
$$

and

$$
q=\hat{q} e^{j \omega t}
$$

the longitudinal impedance of the rigid vessel, $Z_{l}^{b}$, can be derived to be:

$$
Z_{l}^{b}=\frac{-\frac{\hat{\partial p}}{\partial z}}{\hat{q}}=\left[\frac{c_{q}-\left(2-c_{p}\right)}{2-c_{p}}\right] R_{v}+j \omega L\left[\frac{1}{2-c_{p}}\right]+R_{v}
$$

The longitudinal impedance for the electrical analog $Z_{l}^{e}$ in Figure 2.1 is given by:

$$
Z_{l}^{e}=j \frac{\omega R_{1}^{2} L_{1}}{R_{1}^{2}+\omega^{2} L_{1}^{2}}+\frac{\omega^{2} L_{1}^{2} R_{1}}{R_{1}^{2}+\omega^{2} L_{1}^{2}}+R_{2} .
$$

Because the total vessel impedance should converge to a Poiseuille resistance for steady flow in the electrical analog, $R_{2}$ equals a Poiseuille resistance. By comparing the real and imaginary parts of (2.10) and (2.11) the mathematical expressions derived for the model parameters per segment length are derived:

$$
\begin{aligned}
& R_{1}=f(\alpha) R_{v} \\
& L_{1}=g(\alpha) L \\
& R_{2}=R_{v}
\end{aligned}
$$

with

$$
f(\alpha)=\left[\frac{c_{q}-\left(2-c_{p}\right)}{2-c_{p}}+\frac{\alpha^{4}}{64\left(c_{q}-\left(2-c_{p}\right)\right)\left(2-c_{p}\right)}\right],
$$

and

$$
g(\alpha)=\left[64 \frac{\left(c_{q}-\left(2-c_{p}\right)\right)^{2}}{\left(2-c_{p}\right) \alpha^{4}}+\frac{1}{2-c_{p}}\right] .
$$

As can be seen in Figure 2.2, the function $f(\alpha)$ is a smooth curve that goes to infinity for both large and small Womersley numbers. Function $g(\alpha)$ monotonically decreases to 
1 for $\alpha>\sqrt{2}$ and has a constant value 2 for $\alpha \leq \sqrt{2}$. The limiting cases are discussed in the next section where the derived lumped model is compared with Womersley theory and the boundary layer model described by Bessems [61].
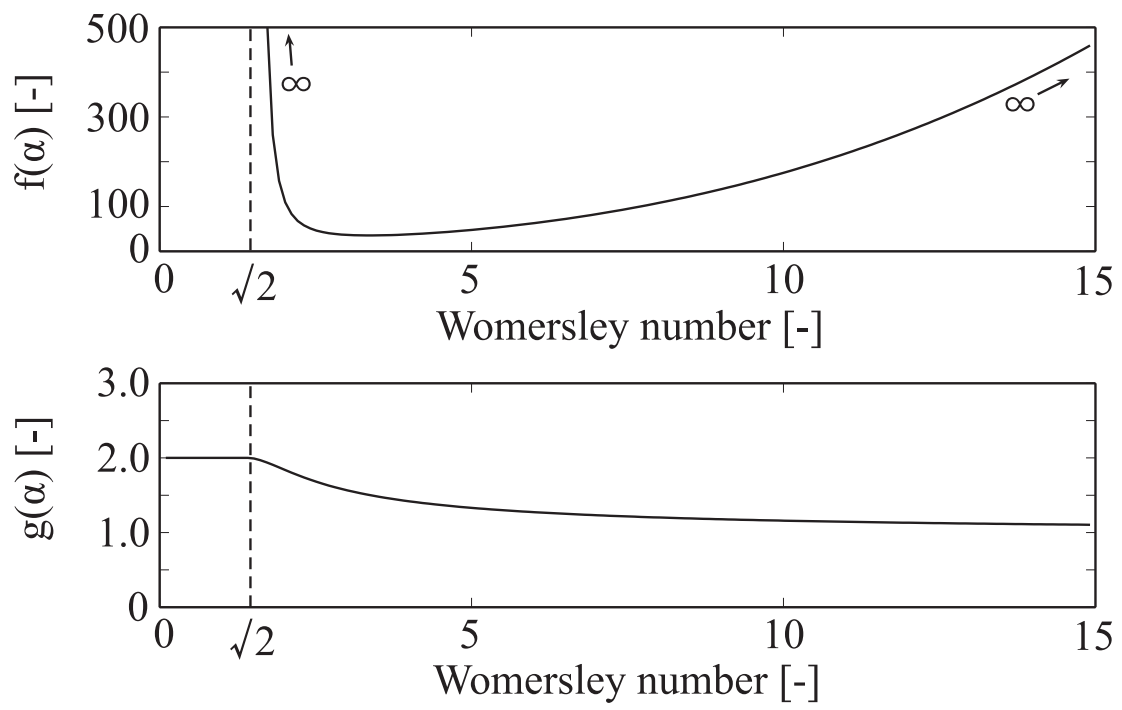

Figure 2.2: The functions $f(\alpha)$ (top) and $g(\alpha)$ (bottom) as a function of the Womersley number. The function $f(\alpha)$ converges to infinity for both $\alpha \rightarrow \infty$ and $\alpha \rightarrow 0$.

\subsection{Results: Comparison between the lumped model, bound- ary layer model and Womersley theory}

\subsubsection{Relative impedance}

In this section, the relative impedance of the proposed electrical analog, the Womersley theory and Bessems' boundary layer model are compared. The relative impedance $I$ is defined as the longitudinal impedance $\left(Z_{l}\right)$ normalized with the Poiseuille resistance $R_{v}$. From the definition of the Womersley number it can be derived that:

$$
\frac{\omega L}{R_{v}}=\frac{\alpha^{2}}{8}
$$


After substitution of (2.12), (2.13), (2.14) and (2.17) in (2.11) the relative impedance for the electrical analog is given by:

$$
I^{e}=\frac{Z_{l}^{e}}{R_{v}}=\frac{j \frac{\alpha^{2}}{8} g(\alpha)}{1+j \frac{\alpha^{2}}{8} \frac{g(\alpha)}{f(\alpha)}}+1 .
$$

The relative impedance for the boundary layer model is:

$$
I^{b}=\frac{Z_{l}^{b}}{R_{v}}=\frac{c_{q}}{2-c_{p}}+j \frac{\alpha^{2}}{8} \frac{1}{2-c_{p}},
$$

while the relative impedance for Womersley reads [84]:

$$
I^{w}=\frac{Z_{l}^{w}}{R_{v}}=j \frac{\alpha^{2}}{8} \frac{1}{1-F_{10}(\alpha)}
$$

in which $F_{10}$ is the Womersley function.

As a reference, the relative impedance is also given for an electrical analog consisting of an inductor and resistor in series (Figure 2.1) as introduced by Noordergraaf [82]:

$$
I^{n}=\frac{Z_{l}^{n}}{R_{v}}=j \frac{\alpha^{2}}{8}+1
$$

In Figure 2.3 the relative impedance of the lumped model is compared with both boundary layer model and Womersley theory. As expected, a perfect match is found for the impedances for the lumped model and the boundary layer model. For both models differences with Womersley theory are very small, i.e., much smaller than for Noordergraaf's model, and only visible in the phase angle for Womersley numbers between 1 and 6 .

\subsubsection{Limiting cases}

To investigate if the limiting behavior of the relative impedances of the proposed electrical analog and Womersley theory are consistent, the limiting cases for a parabolic $(\alpha \ll 1)$ and a flat $(\alpha \gg 1)$ velocity profile are examined.

\section{The relative impedance for large Womersley numbers $(\alpha \gg 1)$}

For $\alpha>\sqrt{2}$ in the boundary layer model $c_{q}$ and $c_{p}$ are defined by (2.7). The relative impedance of our proposed electrical analog yields after substitution of (2.7), (2.15) and 

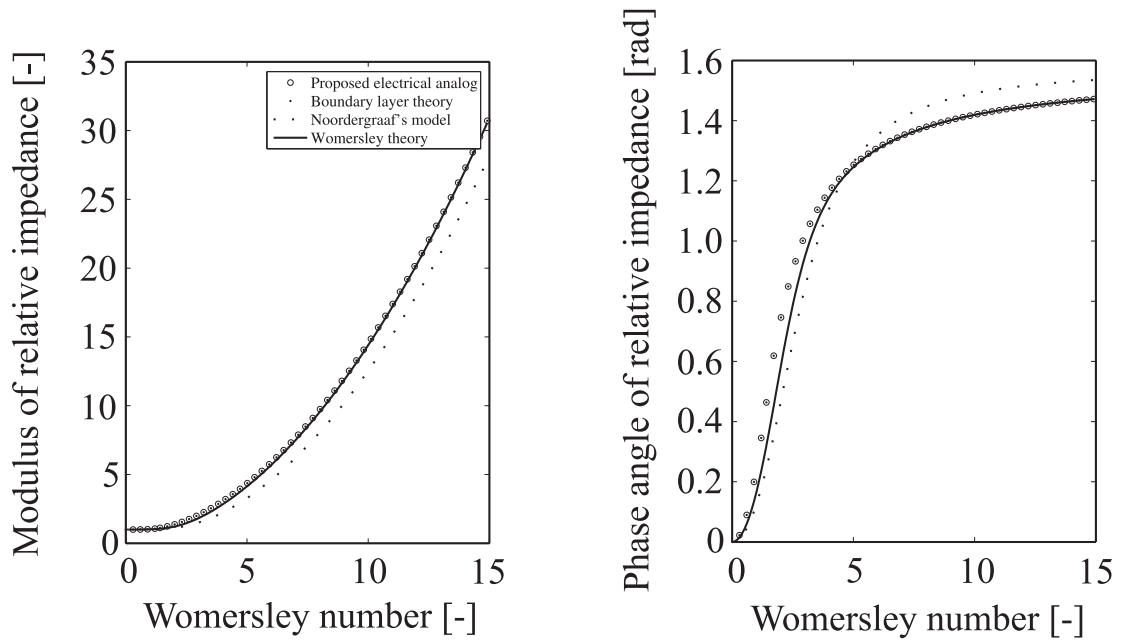

Figure 2.3: The modulus (left) and phase angle (right) of the relative impedance from the lumped model (circles), the boundary layer model (points), Noordergraaf's model (dotted line), and Womersley theory (solid line) as a function of the Womersley number.

(2.16) in (2.18):

$$
I^{e}=\frac{\alpha^{4}}{4 \sqrt{2} \alpha^{3}-12 \alpha^{2}+8 \sqrt{2} \alpha-4}+j \frac{\alpha^{5}}{8 \alpha^{3}-8 \sqrt{2} \alpha^{2}+8 \alpha}
$$

which can be approximated by

$$
I^{e} \approx \frac{\alpha}{4 \sqrt{2}}+j \frac{\alpha^{2}}{8} \quad \text { for } \alpha \gg 1 .
$$

The relative impedance based on Womersley theory is given by (see appendix)

$$
I^{w} \approx \frac{\alpha}{4 \sqrt{2}}+j \frac{\alpha^{2}}{8}, \quad \text { for } \alpha \gg 1 .
$$

The approximate longitudinal impedances for the electrical analog and for Womersley theory can per definition be obtained by multiplying respectively (2.23) and (2.24) with the Poiseuille resistance $R_{v}$. By substituting (2.17) in (2.23) and (2.24), the longitudinal impedances for $\alpha \gg 1$ for both the electrical analog and Womersley theory are after neglecting the real part represented by the longitudinal impedance of an inertance, i.e., a flat 
velocity profile results.

\section{The relative impedance for small Womersley numbers $(\alpha \ll 1)$}

For $\alpha \leq \sqrt{2}$ in the boundary layer model $c_{q}$ and $c_{p}$ are defined as constants and respectively $\frac{1}{2}$ and $\frac{3}{2}$. For $g(\alpha)$ this gives:

$$
g(\alpha \leq \sqrt{2})=2
$$

As the denominator of $f(\alpha)$ is 0 for all Womersley numbers smaller than $\sqrt{2}$, the reciprocal of $f(\alpha)$ for $\alpha \leq \sqrt{2}$ is determined by

$$
\frac{1}{f(\alpha)}=\frac{\left(c_{q}(\alpha)-\left(2-c_{p}(\alpha)\right)\right)\left(2-c_{p}(\alpha)\right) 64}{64\left(c_{q}(\alpha)-\left(2-c_{p}(\alpha)\right)\right)^{2}+\alpha^{4}}=\frac{0}{\alpha^{4}} .
$$

After substitution of the equations (2.25) and (2.26) in (2.18), the relative impedance on the domain $\alpha \in(0, \sqrt{2}]$ is given by

$$
I^{e}=1+j \frac{\alpha^{2}}{4}
$$

For $\alpha \ll 3$ the relative impedance based on Womersley theory is (see appendix)

$$
I^{w} \approx 1+j \frac{\alpha^{2}}{4}
$$

The approximate longitudinal impedances for both the electrical analog and Womersley theory can per definition be obtained by multiplying respectively (2.27) and (2.28) with the Poiseuille resistance $R_{v}$. By using (2.27) and (2.28), the longitudinal impedances for $\alpha \ll 1$ for both the electrical analog and Womersley theory are after neglecting the imaginary part represented by the longitudinal impedance of a Poiseuille resistor, i.e. a parabolic velocity profile results.

\subsubsection{Simplified models}

The resistor $R_{1}$ and the inertia $L_{1}$ in Figure 2.1 are only dependent on the Womersley number as is derived in equation (2.12) and (2.13). It is thus possible to derive simplified lumped models that can be solved in the time-domain by assuming a characteristic Womersley number. In this section we derive simplified lumped models for Womersley numbers smaller than $\sqrt{2}$, as in this case only Poiseuille flow remains in the boundary layer model, and for Womersley numbers larger than $\sqrt{2}$ ). 
Womersley number $\alpha \leq \sqrt{2}$

As was shown previously the function $f(\alpha)$ for Womersley numbers smaller than $\sqrt{2}$ is infinitely large and thus flow through resistor $R_{1}$ is blocked. A simplified model then consists of an inertance of $2 L$ in series with a Poiseuille resistance. If the Womersley number decreases to zero, the relative impedance in (2.27) decreases to $1 . \alpha \leq \sqrt{2}$ the modulus of the relative impedance in (2.27) differs less than $12 \%$ from 1 and because we consider this as not significant, the longitudinal impedance $Z_{l}^{e}$ can be approximated by $R_{v}$. The simplified electrical analog then consists of only a resistor $R_{v}$.

\section{Womersley number $\alpha>\sqrt{2}$}

Functions $f(\alpha)$ and $g(\alpha)$ are plotted in Figure 2.2 for Womersley numbers between $0<\alpha \leq 15$. It can be seen from this figure and equation (2.12) that the resistance $R_{1}$ of the boundary layer increases with increasing Womersley number. This can be explained from the fact that the boundary layer becomes thinner with increasing Womersley number, whereas the central core thickens. The latter can be seen in Figure 2.2 from a decreasing $g(\alpha)$. To come to simplified models for $\alpha>\sqrt{2}$ the ratio between the impedances of $R_{1}$ and $L_{1}$ needs to be studied more thoroughly. By using (2.17) this ratio is given by:

$$
\frac{Z_{R_{1}}}{Z_{L_{1}}}=\frac{R_{v} f(\alpha)}{j \omega L g(\alpha))}=\frac{8 f(\alpha)}{j \alpha^{2} g(\alpha)} .
$$

The modulus and argument of equation (2.29) are given in Figure 2.4. The phase difference is $\frac{\pi}{2}$ radians which is expected as the impedance $Z_{L_{1}}$ is purely imaginary while the impedance of the resistor $Z_{R_{1}}$ is real. The modulus remains larger than one for all Womersley numbers and $Z_{R_{1}}$ is at least eleven times higher than $Z_{L_{1}}$. Based on this observation the resistor $R_{1}$ can be omitted from the proposed electrical analog because the vast majority of the flow will go through the inertance. A simplified electrical analog with an inertance in series with a Poiseuille resistance is then obtained.

\subsection{Discussion}

In this study we aimed to derive a simple lumped model for a vessel segment in which the element configuration is based on physical phenomena described by a boundary layer model and for which all parameters have a physically based quantitative value dependent on the Womersley number.

An electrical analog was derived based on the boundary layer model that was derived by 

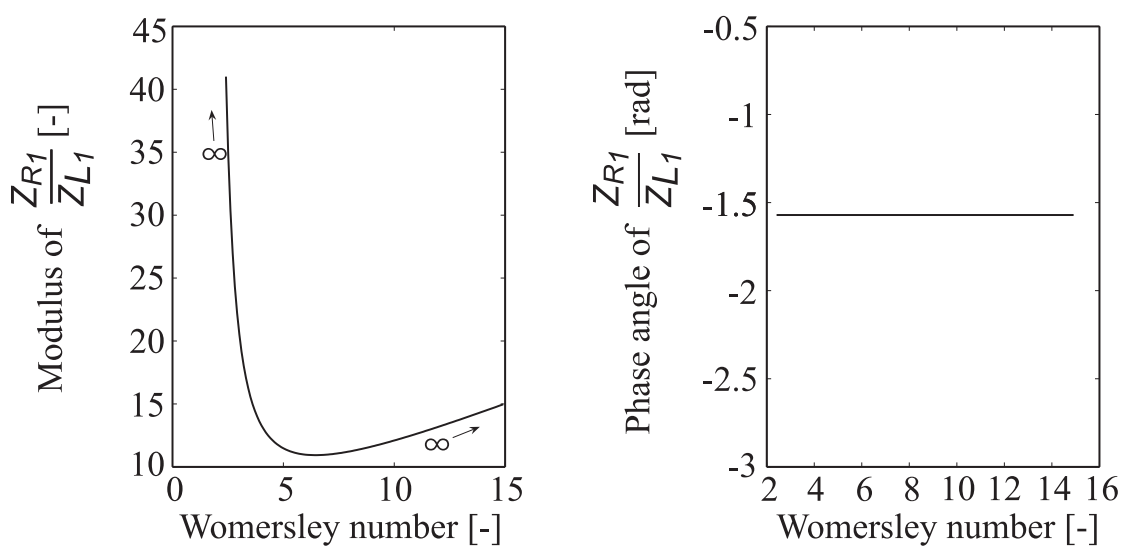

Figure 2.4: The modulus (left) and phase angle (right) of the impedance ratio $\frac{Z_{R_{1}}}{Z_{L_{1}}}$ as a function of the Womersley number.

Bessems et al. [61]. They assumed viscous dominated flow in the boundary layer and inertia dominated flow in the vessel core. Therefore, after neglecting the external forces, the nonlinear term and the diffusion term in the $1 \mathrm{D}$-momentum equation, mathematical expressions were derived for an electrical analog that consists of a parallel arrangement of a resistor and an inertance per segment length that represent respectively the viscous resistance in the boundary layer and the inertia dominated impedance in the central core. In series with those parallelly arranged elements a Poiseuille resistance was introduced so that for steady ( $\alpha=0$ ) viscous flow a Poiseuille resistance is obtained in the electrical analog. The inertia of the core and the resistance of the boundary layer are fully described by fluid density, fluid viscosity, vessel diameter, and Womersley number. The difference between the relative impedances of the proposed lumped model and Womersley theory was very small, i.e. much smaller than for Noordergraaf's model, and only visible in the phase angle for Womersley numbers between 1 and 6 . For both large $(\alpha \gg 1)$ and small $(\alpha \ll 1)$ Womersley numbers, the relative impedance of the proposed model was consistent with Womersley theory.

The model configuration proposed in this study was also found by Olufsen et al. [83] for vessels with a radius between 5 and $15 \mathrm{~mm}$ ( $\alpha$ between approximately 5 and 15). The parameters for their model were $L_{1} \approx \frac{4}{3} L, R_{1} \approx 4 \frac{1}{4} R_{v}$ and $R_{2}=R_{v}$. In our analysis we find a $L_{1}$ that varies between $L$ and $2 L$ depending on the Womersley number; for $\alpha=5$ holds $L_{1}=\frac{4}{3} L$. The resistor $R_{1}$ in our analysis is significantly higher (at least a factor 8 for $\alpha=3$ ) than in the lumped model proposed by Olufsen et al. for all Womersley 
numbers. A high $R_{1}$ is in accordance with the findings of Westerhof et al. as a network of segments consisting of a resistor and inductor in series suffices to model the whole vascular bed [36].

If a capacitor is added to our proposed electrical analog (Figure 2.1), the model consists of the same elements as the four-element windkessel model proposed by Stergiopulos et al. [47]. It thus provides some justification for positioning the additional inertial term in the four-element windkessel model in parallel to the first resistor. Our lumped model suggests that the inertial term represents the inertia-dominated flow in the central core of the vessels, which is supported by Stergiopulos' conclusion that the inductor in the windkessel model represents the total blood inertance of the system. However, the fourelement windkessel model is used to represent the cardiovascular system as a whole, whereas the lumped model in this study is derived by considering flow in a single segment.

By assuming the characteristic frequency (i.e., the harmonic that contains most of the wave energy) and using the mathematical expressions derived in this study, it is possible to derive expressions for segments that can be used to model a rigid tube model in the time-domain. A compliant tube model can be obtained after introducing a capacitor to each of the vessel segments (Figure 2.1). By adding the compliant tube models together in series, it would also be possible to develop a transmission line model of the total arterial tree. However, only a slight improvement of the total vascular impedance is expected compared to a network built from segments only containing a resistor and inductor in series and a capacitor. This because a comparison of the impedance moduli $Z_{L_{1}}$ and $Z_{R_{1}}$ showed that $Z_{R_{1}}$ is at least one order higher and thus can be omitted from the lumped model. This is supported by the findings of Westerhof et al. [36], who incorporated the extra network introduced by Jager [64] in his transmission line model [36], and found that the extra network only slightly improved the input impedance of the systemic arterial tree compared to a network built from segments only containing a resistor and inductor in series and a capacitor. Although the improvements of the input impedance of the transmission line that consists of tube segments proposed in this article are expected to be small, all the electrical elements in such a line will be related to physical phenomena.

\subsection{Conclusion}

We were able to derive a simple lumped model for which all parameters have a physically based quantitative value dependent on the Womersley number and in which the element configuration is based on physical phenomena described by a wave propagation model 
with an approximate velocity profile function. After incorporating a capacitor representing the vessel compliance in this rigid tube model, the element configuration resembles the configuration of the four-element windkessel model. For arbitrary Womersley numbers the relative impedance of Womersley theory is approximated with high accuracy. In the limits for small and large Womersley numbers the relative impedances of the proposed lumped model correspond exactly to Womersley theory.

\section{Appendix: The relative impedance for Womersley theory for small and large Womersley parameters}

The relative impedance $I$ is defined as the longitudinal impedance $\left(Z_{l}\right)$ normalized with the Poiseuille resistance $R_{v}$. From the definition of the Womersley number it can be derived that:

$$
\frac{\omega L}{R_{v}}=\frac{\alpha^{2}}{8} .
$$

After substitution of (2.30) the relative impedance for Womersley reads [84]:

$$
I^{w}=\frac{Z_{l}^{w}}{R_{v}}=j \frac{\alpha^{2}}{8} \frac{1}{1-F_{10}(\alpha)}
$$

in which $F_{10}$ is the Womersley function.

The Womersley function $F_{10}$ is defined as [84]:

$$
F_{10}=\frac{2 J_{1}\left(j^{3 / 2} \alpha\right)}{j^{3 / 2} \alpha J_{0}\left(j^{3 / 2} \alpha\right)}
$$

in which $J_{0}$ and $J_{1}$ are respectively the zero and first-order Bessel functions of the first kind with a complex argument. In the following we will consider the limiting cases of equation (2.31) for small and large values of the Womersley number.

\section{Small Womersley numbers}

To derive the relative impedance $I^{w}$ for small Womersley numbers, the Bessel functions in equation (2.32) are approximated by their power series. Following the power series 
defined in McLachlan [85] the Womersley function can be approximated by

$$
F_{10} \simeq \frac{2 \frac{j^{3 / 2} \alpha}{2}\left[\left(1-\frac{\alpha^{4}}{192}\right)+j\left(\frac{\alpha^{2}}{8}-\frac{\alpha^{6}}{9216}\right)+O\left(\alpha^{8}\right)\right]}{j^{3 / 2} \alpha\left[\left(1-\frac{\alpha^{4}}{64}\right)+j\left(\frac{\alpha^{2}}{4}-\frac{\alpha^{6}}{2304}\right)+O\left(\alpha^{8}\right)\right]} .
$$

From equation (2.33) it can be derived that

$$
\frac{1}{1-F_{10}} \simeq \frac{\left(1-\frac{\alpha^{4}}{64}\right)+j\left(\frac{\alpha^{2}}{4}-\frac{\alpha^{6}}{2304}\right)+O\left(\alpha^{8}\right)}{\left(-\frac{\alpha^{4}}{91}\right)+j\left(\frac{\alpha^{2}}{8}-\frac{\alpha^{6}}{3072}\right)+O\left(\alpha^{8}\right)} .
$$

Next, for $\alpha^{4} \ll 64$ and thus $\alpha \ll 3$, equation (2.34) can be written approximately as

$$
\frac{1}{1-F_{10}} \simeq \frac{1+j \frac{\alpha^{2}}{4}}{j \frac{\alpha^{2}}{8}}
$$

Substitution of (2.35) in (2.32) then gives the relative impedance $I^{w},(2.31)$, for $\alpha \ll 3$ :

$$
I^{w}=1+j \frac{\alpha^{2}}{4}
$$

\section{Large Womersley numbers}

For large Womersley numbers the asymptotic expansions for the expressions $J_{1}\left(j^{3 / 2} \alpha\right)$ and $J_{0}\left(j^{3 / 2} \alpha\right)$ are used [85]. The Womersley function then reads for $\alpha \gg 1$ [85]:

$$
F_{10} \simeq \frac{2}{j^{3 / 2} \alpha} \frac{\frac{e^{\frac{\alpha}{\sqrt{2}}}}{\sqrt{2 \pi \alpha}}\left[\cos \left(\frac{\alpha}{\sqrt{2}}-\frac{\pi}{8}+\frac{\pi}{2}\right)+j \sin \left(\frac{\alpha}{\sqrt{2}}-\frac{\pi}{8}+\frac{\pi}{2}\right)\right]}{\frac{e^{\frac{\alpha}{\sqrt{2}}}}{\sqrt{2 \pi \alpha}}\left[\cos \left(\frac{\alpha}{\sqrt{2}}-\frac{\pi}{8}\right)+j \sin \left(\frac{\alpha}{\sqrt{2}}-\frac{\pi}{8}\right)\right]} .
$$

Because $j^{3 / 2}=e^{j \frac{3 \pi}{4}}$ and $j^{-1 / 2}=e^{-j \frac{\pi}{4}}=\frac{(1-j)}{\sqrt{2}}$ the expression in (2.37) can be simplified to

$$
F_{10} \simeq \frac{2}{\alpha} e^{-j \frac{\pi}{4}}=\frac{(1-j) \sqrt{2}}{\alpha} .
$$

Substituting (2.38) in (2.32) gives the relative impedance $I^{w},(2.31)$, for $\alpha \gg 1$ :

$$
I^{w}=j \frac{\alpha^{2}}{8} \frac{1}{1-\frac{(1-j) \sqrt{2}}{\alpha}}=\frac{\alpha^{4}}{4 \sqrt{2} \alpha^{3}-16 \alpha^{2}+16 \sqrt{2} \alpha}+j \frac{\alpha^{5}-\sqrt{2} \alpha^{4}}{8 \alpha^{3}-16 \sqrt{2} \alpha^{2}+32 \alpha} .
$$




\section{Chapter 3}

\section{Pulse wave propagation model}

This Chapter is based on:

W. Huberts, A.S. Bode, W. Kroon, R.N. Planken, J.H.M. Tordoir, F.N. van de Vosse, E.M.H Bosboom, A pulse wave propagation model to support decision-making in vascular access planning in the clinic, Med. Eng. Phys., Online available, doi: 10.1016/j.medengphy.2011.07.015, (2011) 


\begin{abstract}
The preferred vascular access for hemodialysis is an autologous arteriovenous fistula (AVF) in the arm: a surgically created connection between an artery and vein. The surgeon selects the AVF location based on experience and preoperative diagnostics. However, 20-50\% of all lower arm AVFs are hampered by a too low access flow, whereas complications associated with too high flows are observed in $20 \%$ of all upper arm AVFs. We hypothesize that a pulse wave propagation model fed by patient-specific data has the ability to assist the surgeon in selecting the optimal AVF configuration by predicting direct postoperative flow.

Previously, a 1D wave propagation model (spectral elements) was developed in which an approximated velocity profile was assumed based on boundary layer theory. In this study, we derived a distributed lumped parameter implementation of the pulse wave propagation model. The elements of the electrical analog for a segment are based on the approximated velocity profiles and dependent on the Womersley number. We present the application of the lumped parameter pulse wave propagation model to vascular access surgery and show how a patient-specific model is able to predict the hemodynamical impact of AVF creation and might assist in vascular access planning.

The lumped parameter pulse wave propagation model was able to select the same AVF configuration as an experienced surgeon in nine out of ten patients. In addition, in six out of ten patients predicted postoperative flows were in the same order of magnitude as measured postoperative flows. Future research should quantify uncertainty in model predictions and measurements.
\end{abstract}




\subsection{Introduction}

In Europe, more than 500.000 end-stage renal disease (ESRD) patients are dependent on hemodialysis treatment [3]; a number that is expected to increase annually by approximately $8 \%[4,5]$. During hemodialysis, the blood of the patient is withdrawn from the body using a pump with a flow rate of at least $300 \mathrm{ml} / \mathrm{min}$ and directed through an artificial kidney (dialyzer) where excess fluid, minerals and metabolic waste products are removed from the blood. A few centimeters proximal to the outlet, the blood is pumped from the dialyzer back into the body with the same flow rate (Figure 3.1). To enable hemodialysis a functioning vascular access (VA) is needed. The VA should deliver the high flow needed for extracorporeal circulation and should have a caliber that enables, over time, repetitive cannulation to connect the patient with the dialyzer [6, 7]. The human body has no blood vessel that meets all these requirements without interventions. Therefore a VA is surgically created. Usually, a VA is created in the arm by permanently connecting an artery and a vein, i.e an arteriovenous fistula (AVF, see Figure 3.1). In this way the peripheral resistance is bypassed, which results in a significant blood flow increase (five- to thirtyfold) and vessel remodeling.The AVF is considered properly matured if at six weeks the access flow is sufficiently high ( $>600 \mathrm{ml} / \mathrm{min}$ ), the proximal vein is sufficiently dilated (diameter $>6 \mathrm{~mm}$ ) and the VA is located superficial enough (depth $<6$ $\mathrm{mm})[6,7,8,9,10]$. A matured AVF is able to facilitate adequate hemodialysis treatment and repetitive cannulation. An AVF can be created at several locations in the arm. The chosen site is a compromise between initial and long-term access patency, complication rates and saving potential access sites for future procedures [11, 12, 13, 14]. For the former reasons, the AVF at wrist level is preferred over more proximal AVFs which involve an anastomosis at the elbow level. In clinical practice, the surgeon selects the AVF configuration based on experience. A preoperative vessel assessment is used to determine the continuity and caliber of the vessels. Arterial pulsatility and the continuity of both arteries and veins are assessed by palpation. Additionally, more vessel details are obtained by duplex ultrasound measurements $[7,8,31]$. Despite the expertise of the surgeon and the extensive preoperative work-up, non-maturation occurs in up to $50 \%$ of all newly created lower arm AVFs $[6,7,10,12]$. On the other hand, complications associated with too high flows are observed in $20 \%$ of all upper arm AVFs [6, 7, 8, 12]. A too low flow measured directly after surgery is indicative for non-maturation [10, 32], while a high postoperative flow ( $>30 \%$ of the cardiac output) increases the risk on cardiac failure and hand ischemia $[11,12,13,14]$. A tool that can preoperatively predict direct postoperative flow is potentially clinically important to enable better selection of the AVF configuration. 
Procedure for an upper arm AVF:

Torso and arm vasculature
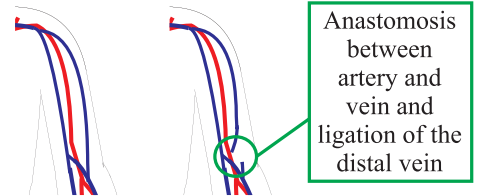

before surgery directly after surgery

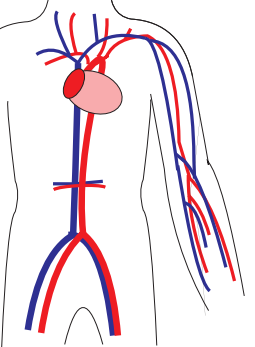

Procedure for a lower arm AVF:

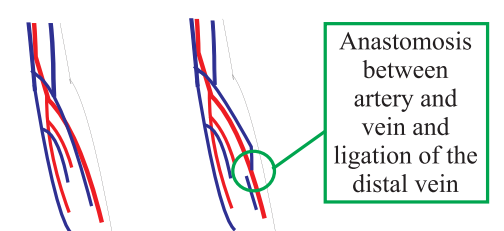

before surgery directly after surgery

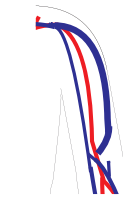

after six week (maturation)

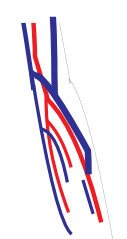

after six weeks

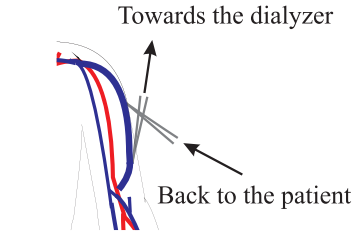

during hemodialysis session

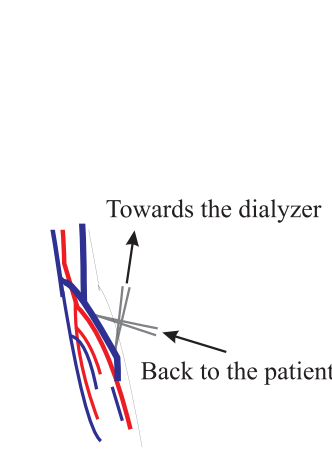

during hemodialysis session
Anastomosis:

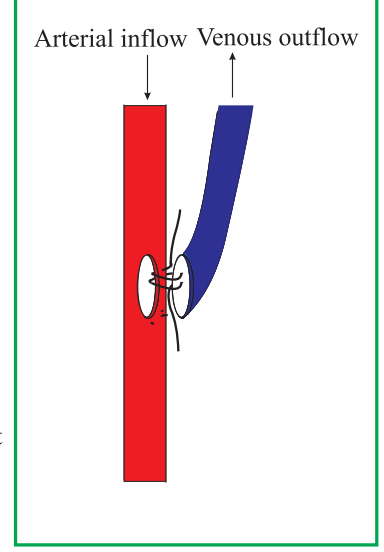

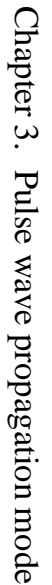

Figure 3.1: A schematic picture of the torso and arm vasculature including the major arteries and the superficial veins (left). The procedure of creation of an arteriovenous fistula (permanent connection between the artery and the vein) in the upper (middle, top) and lower (middle, bottom) arm and the connection of the VA to the dialyzer are depicted. At the right the anastomosis is shown in more detail. 
We hypothesize that a computational model fed by patient-specific data is able to predict direct postoperative flow and has the ability to assist the surgeon in selecting the optimal AVF configuration. The advantage of a computer model is that different factors that influence VA flow (e.g. diameter, accessory veins and vessel distensibility) and their mutual dependency can be taken into account.

Computer models with various degrees of complexity ranging from $3 \mathrm{D}$ models $[38,53$, 54], pulse wave propagation models (cited below), to lumped parameter models [34, 46, $47,56,57]$ are used to study the (patho-)physiology of the cardiovascular system, depending on the application of interest. Although 3D models enable for studying local flow phenomena, they are less suitable for predictive surgery because of their high computational cost. Pulse wave propagation models are appropriate for predictive vascular surgery, because they can be used to calculate the distribution of pressure and flow throughout the vascular system within minutes. And besides, pulse wave propagation models are easier to adapt to patient-specific conditions than 3D methods. Lumped parameter models might also be applied in predictive surgery, however, in contrary to pulse wave propagation models, geometric information is lost, while the latter can be essential when planning surgical interventions. Moreover, in pulse wave propagation models nonlinear equations can be incorporated describing the relation between cross-sectional area and pressure $[50,51,52,61,62]$, or describing additional pressures drops resulting from stenoses, curvature and anastomoses $[50,51,52,62]$. In our application, i.e. on vascular access surgery, a nonlinear flow waveform dependent relation is needed to incorporate the pressure drop over the anastomosis. For these reasons, a pulse wave propagation model is chosen in this study. Although some previous studies have described the use of pulse wave propagation models to predict hemodynamic changes after creation of femoro-popliteal [50], thoraco-thoraco aortic [51, 52] and aorto-femoral bypassess [52], the use of predictive models is not common in clinical practice yet.

Pulse wave propagation models include the multi-branched configuration of the arterial system and a description of the distributed nature of arterial properties. The vascular system is divided into segments that represent local blood and vessel wall properties. All segments are serially connected based on the anatomical configuration. For each segment the relation between pressure and flow is described by the one-dimensional momentum and continuity equations derived by Hughes and Lubliner [59]. In addition, a constitutive law is used to describe wall behavior. The resulting equations can be solved for pressure and flow by several numerical techniques like the method of characteristics [33, 60], the spectral element method [61,63] and the finite element method [51,52] resulting in one-dimensional (1D) pulse wave propagation models. Pulse wave propagation models 
in which segments are lumped (spatial information within each segment is lost) into an electrical analog are also used $[36,40,64]$ and here referred to as distributed lumped parameter pulse wave propagation models.

In the momentum equation, approximations for wall shear stress and the convective acceleration term are needed. These terms can be estimated from a large variety of velocity profiles based on Poiseuille [51, 52], Witzig-Womersley theory for pulsatile flow [60], the Young and Tsai formulation [25], and approximated velocity profiles [61, 62]. Bessems et al. $[61,63]$ used an approximated velocity profile based on boundary layer theory and solved the resulting set of equations in time domain by using spectral elements.

Previously, we showed that after neglecting the nonlinear convection term, it is possible to derive a lumped parameter segment [86] to describe the pressure-flow relation in each vascular segment based on the set of equations used by Bessems [61] without loosing accuracy. Neglecting the convection term is reasonable, as in the application in this study this term is expected to be relatively small $[65,87]$ compared to the inaccuracy of the flow predictions resulting from inaccuracies in input parameters. Numerical implementation of a wave propagation model consisting of lumped parameter segments is straightforward. This method defines flow positive when directed into the segment and therefore no coupling equations are needed. Furthermore, the lumped parameter wave propagation model simplifies coupling of other lumped segments (e.g. for windkessels or for nonlinear elements like the anastomosis) to wave propagation segments because they have the same format and can be assembled together. In addition, the lumped parameter wave propagation implementation is, in our experience, robust. A lumped parameter description is intuitive and comprehensible, as the lumped parameter segment is build of three types of elements; resistors representing resistance to blood flow, inductors related to the force that is needed to accelerate blood and capacitors representing the storage capacity of the blood vessel. We thus expect that a lumped parameter pulse wave propagation model will be more readily implemented and used in clinical practice. In this study we derive and present a lumped parameter pulse wave propagation model and, in addition, we show how the model can be made patient-specific and might be used for VA surgery planning. The difference between the lumped model approach in this study and the one in for example Westerhof et al. [36] is that our electrical elements are based on an approximated velocity profile, which result in Womersley number dependent resistors and inductors. We do not need to include extra electrical elements to describe Womersley profiles as in Westerhof et al. [36]. Another main difference is that we allow for vessel diameter tapering within one segment.

We will first describe the mathematical approach of the pulse wave propagation model 
and the modeling of the feeding artery, the anastomosis and the outflow veins of the AVF complex. Next to the mathematical description, the interpretation of the different elements is given to improve the understanding of the model's background. Subsequently, the model is applied to VA surgery and we examine whether the model is able to delineate flow increases in upper arm and lower arm AVFs. Finally, we will compare simulations results of flow after VA creation with flows observed in a clinical setting.

\subsection{Material and methods}

\subsubsection{The mathematical model}

For the scope of modeling the effect of VA on blood pressure and flow distribution, segments are needed that represent arteries, veins and the anastomosis. By combining the segments we are able to construct different vascular topologies with or without an AVF. Before we describe the patient-specific topologies in detail, we will first present the physical and mathematical background of the different segments and how the segments can be assembled to form the distributed lumped parameter wave propagation model.

\section{Governing equations}

The relation between pressure $p$ and flow $q$ for each vascular segment is derived from conservation of mass and the momentum equation by assuming fully-developed incompressible Newtonian flow in a straight vessel. For more details we refer to $[59,61]$ and the Appendix of this chapter. The mass equation is further simplified by assuming a compliance $C_{0}$ per unit length, resulting in

$$
C_{0} \frac{\partial p}{\partial t}+\frac{\partial q}{\partial z}+\Psi=0
$$

with

$$
C_{0}=\left.\frac{\partial A}{\partial p}\right|_{p=p_{0}}
$$

in which $p_{0}$ is the mean pressure, $A$ is the cross-sectional area and $\Psi$ the flow per unit length distributed to small side-branches that are not separately modeled by vascular segments. 
The momentum equation is given by

$$
\frac{\partial q}{\partial t}+\frac{\partial}{\partial z}\left(\int_{A} v_{z}^{2} d A\right)+\frac{A}{\rho} \frac{\partial p}{\partial z}-A f_{z}-\frac{2 \pi a_{0}}{\rho} \tau_{w}=0
$$

in which $A$ is the cross-sectional area, $v_{z}$ the local axial velocity, $a_{0}$ the vessel radius at mean pressure, $\tau_{w}$ the wall shear stress and $\rho$ the blood density. In momentum equation (3.3), the convection term $\left(\frac{\partial}{\partial z}\left(\int_{A} v_{z}^{2} d A\right)\right)$ and the effect of body forces $\left(A f_{z}\right)$ are neglected because their contributions are expected to be small in the arm vasculature, which are the most important vessels in the application for VA planning [65, 87]. Neglecting the convection term is allowed as long as $\frac{V}{c} \ll 1$ in which $V$ is the blood velocity and $c$ the wave speed [55]. This holds in the arm vasculature and by approximation in the aorta.

An expression for wall shear stress as function of $p$ and $q$ is derived from a time and frequency dependent approximated velocity profile which is based on boundary layer theory (see this chapter's Appendix and [61, 86] for more details). The momentum equation then becomes

$$
-\frac{\partial p}{\partial z}=\left[\frac{c_{q}}{2-c_{p}}\right] R_{0} q+\left[\frac{1}{2-c_{p}}\right] L_{0} \frac{\partial q}{\partial t}
$$

in which $R_{0}$ is the Poiseuille resistance per unit length $\left(\frac{8 \eta}{\pi a_{0}^{4}}\right)$ and $L_{0}$ is the inertia per unit length $\left(\frac{\rho}{\pi a_{0}^{2}}\right)$. Parameters $c_{p}$ and $c_{q}$ solely dependent on the Womersley number ( $\alpha=a_{0} \sqrt{\frac{\omega \rho}{\eta}}$ with $\omega$ angular frequency) and are given by [61]

$$
c_{p}=1+\frac{\sqrt{2}}{\alpha}\left(1-\frac{\sqrt{2}}{2 \alpha}\right), \quad c_{q}=\frac{\alpha}{4 \sqrt{2}}\left(1-\frac{\sqrt{2}}{2 \alpha}\right)^{-1} \quad \text { for } \quad \alpha>\sqrt{2}
$$

and

$$
c_{p}=\frac{3}{2}, \quad c_{q}=\frac{1}{2} \quad \text { for } \quad \alpha \leq \sqrt{2}
$$

A lumped parameter segment is derived that consists of a Womersley number dependent resistor per unit length $R$ and a Womersley number dependent inductor per unit length $L$ in series representing the momentum equation (Figure 3.2, left). The capacitor per unit length $C$ and the resistor $R_{L}$ of this lumped parameter segment represent the continuity equation. 


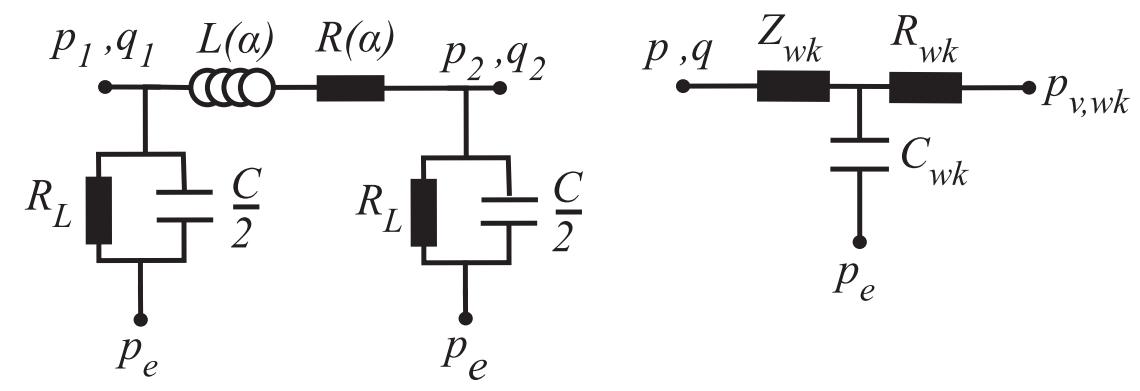

Figure 3.2: Lumped parameter model of a vascular segment (left) and a three-element windkessel model that is used to model branches that are truncated (right).

By evaluating $c_{p}$ and $c_{q}$ only for the characteristic frequency, this serial configuration fits Womersley theory better than the parallel configuration proposed earlier by us [86], whereas when all frequencies are considered they behave similar. For more details we refer to the Appendix of this chapter. By evaluating $c_{p}$ and $c_{q}$ only for the characteristic frequency, parameters $L$ and $R$ stem from momentum equation (3.4), which results in

$$
-\frac{\partial p}{\partial z}=R q+L \frac{\partial q}{\partial t}
$$

with

$$
R=\left[\frac{c_{q}\left(\alpha_{0}\right)}{2-c_{p}\left(\alpha_{0}\right)}\right] R_{0} \quad \text { and } \quad L=\left[\frac{1}{2-c_{p}\left(\alpha_{0}\right)}\right] L_{0}
$$

herein, $\alpha_{0}$ is the Womersley number corresponding to the characteristic frequency.

\section{Vascular segment}

For both arterial and venous segments (Figure 3.2), a lumped parameter segment with the serial arrangement of the resistor and inductor is used. For each segment total resistance and total inertance are obtained by integrating $R$ and $L$ over the segment length.

The superficial veins that are used for venous drainage in an AVF can have an elliptical cross-sectional area and therefore different expressions for $L_{0}$ and $R_{0}$ in (3.8) are used that apply to elliptical tubes. The Poiseuille resistance per segment length for an elliptical tube $R_{0, v}$ is than given by [88]

$$
R_{0, v}=\frac{8 \eta\left(a_{0}^{2}+b_{0}^{2}\right)}{2 \pi\left(a_{0}^{3} b_{0}^{3}\right)}
$$


with $a_{0}$ and $b_{0}$ the major and minor radii at the mean pressure $p=p_{0}$. Note that equation (3.9) reduces to $R_{0}=\frac{8 \eta}{\pi a_{0}^{4}}$ (Poiseuille resistor) in case $a_{0}=b_{0}$. The venous inertance per unit length, $L_{0, v}$, is

$$
L_{0, v}=\frac{\rho}{\pi a_{0} b_{0}} .
$$

To incorporate vessel compliance in the lumped parameter model, a capacitor is added to each side of the vascular segment (Figure 3.2), representing half of the total vascular compliance over that segment [36]. Furthermore, it is assumed that the artery is a thickwalled linear elastic tube. The compliance per segment length is then given by [64]

$$
C_{0}=\frac{2 \pi a_{0}^{2}\left(\frac{2 a_{0}^{2}\left(1-\mu^{2}\right)}{h_{0}^{2}}+(1+\mu)\left(\frac{2 a_{0}}{h_{0}}+1\right)\right)}{E_{0}\left(\frac{2 a_{0}}{h_{0}}+1\right)}
$$

with $E_{0}$ the Young's modulus, $\mu$ the Poisson ratio and $h_{0}$ the wall thickness. Veins can have an elliptical cross-sectional area, and therefore, instead of $a_{0}$ a weighted average radius $\left(\bar{a}_{0}=\sqrt{a_{0} b_{0}}\right)$ is used in (3.11). Because veins are much thinner than arteries, the limit of (3.11) for $\bar{a}_{0}>>h_{0}$ is used for veins, resulting in

$$
C_{0, v}=\frac{2 \pi \bar{a}_{0}^{3}\left(1-\mu^{2}\right)}{E_{0, v} h_{0}}
$$

in which $E_{0, v}$ is the venous Young's modulus. Total compliance for each segment is determined by integrating $C_{0}$ or $C_{0, v}$ over the segment length.

The distributed flow $\Psi$ in (3.1) is captured by adding linear resistances in parallel to the capacitors (Figure 3.2).

\section{Modeling the anastomosis}

The anastomosis (Figure 3.1) cannot be modeled by linear segments because the radial velocity component is no longer infinitesimally small compared to the axial velocity and flow separation can occur in this region. The velocity profile can thus not be based on fully developed flow in straight vessels. Consequently, a special segment is created to model pressure losses over the anastomosis.

Due to complex flow patterns, resulting from the geometry at the anastomotic region and possible turbulence, there is no analytical relation that describes the pressure losses. Steele et al. [51] and Jones et al. [89] have applied pressure loss relations for a T-junction to model the anastomosis of a thoraco-thoraco aortic bypass graft and a hemodialysis 
prosthetic graft, respectively. They both neglected the extra pressure drop due to unsteadiness of flow. This assumptions seems reasonable as pulsatility in an AVF decreases with respect to the steady component and because the cross-sectional area of the vein is larger than the area of the anastomosis which results in deceleration of flow and thus an increase in pressure which might compensate for the extra pressure drop resulting from the unsteady flow. However, future research should investigate this statement. In this study, we therefore followed the same approach as Steele et al. [51] and Jones et al. [89] to model the anastomosis of an AVF (Figure 3.3). Pressure in the proximal artery $p_{p}$ minus pressure in the vein $p_{v}$ and pressure in the proximal artery $p_{p}$ minus pressure in distal artery $p_{d}$ can be written as $[51,90]$

$$
p_{p}-p_{v}=K_{v} \frac{1}{2} \rho v_{p}^{2}
$$

and

$$
p_{p}-p_{d}=K_{d} \frac{1}{2} \rho v_{p}^{2}
$$

respectively. Herein $v_{p}$ is the cross-sectional averaged blood velocity in the proximal artery, and $K_{v}$ the loss coefficient from the proximal artery to the proximal vein, and $K_{d}$ the loss coefficient from the proximal artery to the distal artery.

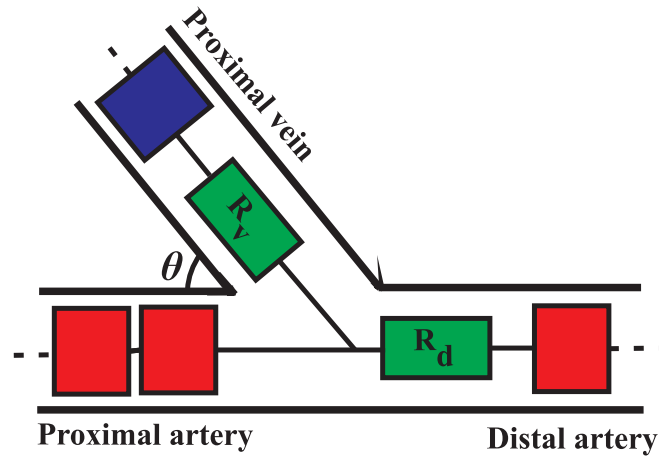

Figure 3.3: The anastomosis incorporated in the lumped parameter pulse wave propagation model.

Gardel et al. [91, 92] experimentally derived semi-empirical relations for the loss coefficients $K_{v}$ and $K_{d}$. The loss coefficients for an anastomosis are given by (dividing 
T-junction) $[90,91,92]$

$$
\begin{gathered}
K_{v}=k_{1}\left(1-q^{*}\right)^{2}+q^{* 2}\left[k_{2} \cot \frac{\theta}{2}-k_{3}+\frac{k_{4}-k_{5} A^{*}}{A^{* 2}}\right]+k_{6} q^{*}\left(1-q^{*}\right)\left(1+\frac{1}{A^{*}}\right) \cot \frac{\theta}{2} \\
K_{d}=k_{7}\left(1-q^{*}\right)^{2}+k_{8} q^{* 2}-k_{9} q^{*}\left(1-q^{*}\right)
\end{gathered}
$$

in which $q^{*}$ is the ratio between flow through the vein and flow through the proximal artery, $A^{*}$ is the ratio between cross-sectional area of the vein and cross-sectional area of the proximal artery and $\theta$ is the angle between proximal artery and proximal vein (Figure 3.3). Empirical determined values for the constants $k_{1}$ to $k_{9}$ are given in Table 3.1.

Table 3.1: Semi-empirical determined dimensionless constants $k_{1}$ to $k_{9}$ for equation (3.15) and (3.16) $[91,92]$.

$\begin{array}{ccccccccc}k_{1} & k_{2} & k_{3} & k_{4} & k_{5} & k_{6} & k_{7} & k_{8} & k_{9} \\ 0.95 & 1.3 & 0.3 & 0.4 & 0.1 & 0.4 & 0.03 & 0.35 & 0.2\end{array}$

By introducing flow $q_{p}=v_{p} A_{p}$ with $A_{p}$ the cross-sectional area of the proximal artery, (3.13) and (3.14) can be rewritten to

$$
p_{p}-p_{v}=K_{v} \frac{1}{2} \rho \frac{q_{p}^{2}}{q_{v}^{2}} \frac{1}{A_{p}^{2}}\left|q_{v}\right| q_{v}=R_{v}\left(q^{*}, A^{*}, \theta, q_{v}, q_{p}, A_{p}\right) q_{v}
$$

and

$$
p_{p}-p_{d}=K_{d} \frac{1}{2} \rho \frac{q_{p}^{2}}{q_{d}^{2}} \frac{1}{A_{p}^{2}}\left|q_{d}\right| q_{d}=R_{d}\left(q^{*}, A^{*}, \theta, q_{d}, q_{p}, A_{p}\right) q_{d}
$$

The pressure drop in the anastomosis from the proximal artery to proximal vein and to distal artery is incorporated in the pulse wave propagation model by adding two resistors, $R_{v}$ and $R_{d}$, that nonlinearly depend on flow.

\section{Numerical scheme}

After assembly of the segments and by using the (implicit) trapezoidal rule for time integration the following system of equations is derived (see this chapter's Appendix for more details)

$$
\underline{K}_{\sim}^{n}=\underline{q}_{e}^{n}+{\underset{\sim}{f}}^{n-1}
$$


in which $\underline{K}$ is the system matrix, $\underline{\sim}^{n}$ is a column containing the nodal pressures that we calculate in the current time step $n, q_{e}^{n}$ denotes the external nodal flows containing zero values for all nodes except the nodes on which external flow is prescribed, and $f^{n-1}$ is the right hand side column vector resulting from time integration and matrix assembly. The system of equations is solved with a direct solver after defining boundary conditions as described in the next section. The flow through each segment is depicted by applying an analogue of Ohm's law.

By incorporating flow dependent resistors (3.17) and (3.18) for the anastomosis, the system becomes nonlinear and is given by

$$
\underline{K}_{i}\left(q_{i}^{*}, A^{*}, \theta, q_{v_{i}}, q_{d_{i}}, q_{p_{i}}, A_{p}\right){\underset{\sim}{p}}_{i}^{n}={\underset{\sim}{q}}_{e}^{n}+{\underset{\sim}{f}}^{n-1} .
$$

To solve (3.20) an iterative procedure, with iterative steps $i$, is used which is described in detail in this chapter's Appendix.

\section{Boundary conditions}

Prescribed flows. In our model aortic flow is prescribed at node $\mathrm{k}=1$ and thus $\underset{\sim}{q_{e}}=$ $\left[q_{\text {aorta }}, 0, . ., 0\right]^{T}$. The aorta is part of the modeling domain to be able to predict the redistribution of flow through the body after AVF creation. Moreover, reflections at the inlet are better represented (closed aortic valve) than if we prescribe the flow at the arm.

Prescribed pressures. In addition, extravascular pressure $p_{e}$ and venous pressure at the end of the venous branch $p_{v}$ and at the end of the windkessel model $p_{v, w k}$ are prescribed.

Windkessel model. At arterial ends, arteries are terminated with a three-element Windkessel model (Figure 3.2) consisting of a characteristic impedance, $Z_{w k}$, a resistance, $R_{w k}$ and a compliance, $C_{w k}$ [57]. The characteristic impedance is chosen such that reflections from high frequencies $\left(\omega C_{w k} R_{w k} \gg 1\right)$ are attenuated [57]. The pressure drop over the characteristic impedance is then given by

$$
p-p_{s}=Z_{w k} q \quad \text { with } \quad Z_{w k}=\sqrt{\frac{L}{C_{0}}}
$$

in which $Z_{w k}$ is the characteristic impedance for $\omega L \gg R$ and $p_{s}$ a standard reference pressure. $R, L$ and $C_{0}$ are, respectively, the resistance, the inertance and the compliance of the segment before the end-segment. 
The sum of the resistance $R_{w k}$ and the characteristic impedance $Z_{w k}$ is the total resistance $R_{p}$ of the peripheral bed [57]:

$$
R_{p}=Z_{w k}+R_{w k}
$$

$R_{p}$ is defined as:

$$
R_{p}=\frac{\bar{p}}{\beta \bar{q}}
$$

with $\beta$ the fraction of the cardiac output flowing through the peripheral bed and $\bar{p}$ the mean arterial pressure and $\bar{q}$ the cardiac output.

The pressure wave decreases during diastole with a time constant that is equal to:

$$
\tau=R_{w k} C_{w k}
$$

If $\tau$ and $R_{w k}$ are known, the compliance $C_{w k}$ can be derived from (3.24). The parameter $R_{w k}$ can be derived from equation (3.22) and (3.23), while $\tau$ can be obtained from the descending slope of the local pressure curve during diastole or can be based on literature.

To adapt the model to patient-specific conditions, blood properties (viscosity, density), and geometrical (radius, wall thickness-to-radius ratio, vessel length, anastomosis angle) and mechanical vessel properties (Poisson ratio, Young's modulus) are needed. Additionally, patient-specific boundary conditions should be available.

\subsubsection{Models for vascular access surgery}

Ten patient-specific models were made using data from ten ESRD-patients awaiting VA creation to facilitate hemodialysis. For all patients the target extremity did not have an earlier VA. The study was approved by the Medical Ethical Committee of the Maastricht University Medical Center (MUMC) and all patients gave their written informed consent. Depending on the arm in which the VA is created, the left or right arm is modeled in detail. The aorta, the vessels in the lower body, in the contralateral arm and in the head are only modeled partially and then closed by windkessel models. These arteries are included into the model to obtain the correct flow distribution over the vasculature as this is unknown after AVF creation. Postoperatively, an element that models the anastomosis and a venous branch are added to model the venous return of the AVF. For an upper arm AVF a single vein is added (basilic or cephalic vein), whereas for a lower arm AVF the distal cephalic 
vein, the median cubital vein and the proximal basilic vein are added. The computational domains of the preoperative, the lower arm and the upper arm configuration are shown in the case of a left arm in Figure 3.4. In case of a right arm configuration, the computational domain is comparable; the left arm is truncated after the first edge (left subclavian artery A) and the right carotid artery and the right arm are added to the end of the innominate artery. The models are made patient-specific as described in the next paragraph. 

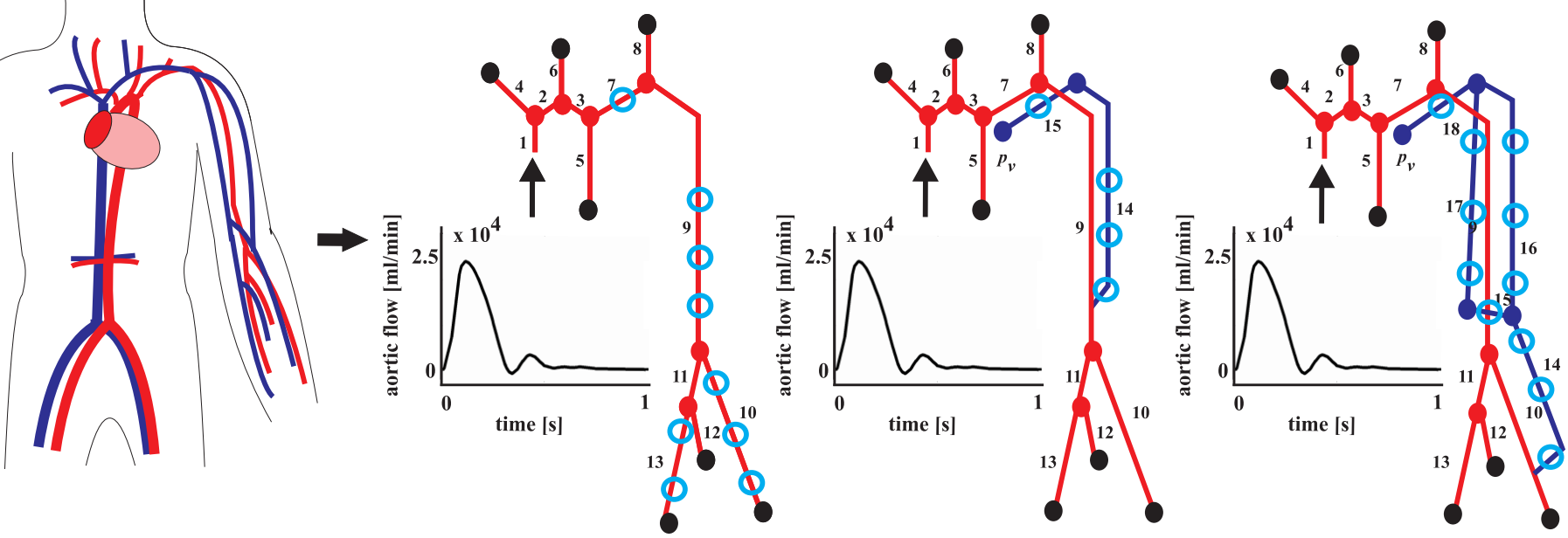

Figure 3.4: The computational domains for the preoperative situation, with an upper arm and with a lower arm AVF in the left arm. The arterial tree (red) and the included veins (blue) are divided in edges. The arterial edge numbers refer to the edges in Table 3.3. The venous edges for the lower arm AVF represent the distal cephalic vein (\#14), the median cubital vein (\#15), the proximal cephalic vein (\#16), the basilic vein and the distal axillary artery (\#17) and the proximal axillary and subclavian vein (\#18). The venous edges for the upper arm AVF represent the proximal cephalic vein and the distal axillary vein (\#14) and the proximal axillary and subclavian vein (\#15). The light blue circles indicate the locations on which diameter measurements are available. 


\section{Patient-specific input}

Model geometry. All ten patients underwent a preoperative duplex ultrasound examination to determine vessel diameters as part of standard clinical health care. Diameters of the brachial, radial and ulnar arteries as well as the basilic, subclavian and cephalic vein were measured in the distal lower arm (a few $\mathrm{cm}$ proximal to the wrist joint), halfway the lower arm, in the proximal lower arm (a few $\mathrm{cm}$ distal to the elbow joint), at the elbow joint, in the distal upper arm (a few cm proximal to the elbow joint), halfway the upper arm and in the proximal upper arm (a few $\mathrm{cm}$ distal to the axilla). In addition, diameters of the subclavian artery and the median cubital vein are determined (Table 3.2). Prior to measurement of the distal venous diameters a tourniquet was applied around the upper arm to induce venous dilatation which increases the reproducibility of the measurement [93, 94]. Arterial and venous diameters were measured in B-mode, both in longitudinal and transversal imaging plane using an electronic caliper with an accuracy of $0.1 \mathrm{~mm}$. Because the ultrasound examination supplies diameters on discrete locations (Figure 3.4), a linear inter- and extrapolation was performed within each vascular edge to obtain diameter-length relations for each vascular edge. All edges are then divided in vascular segments. For diameters of the other vessels, data from Stergiopulos et al. [25] are used (Table 3.3) after applying a patient-specific scaling factor, determined from the ratio between the measured arm diameters and arm diameters from [25]. Also the arterial lengths are based on Stergiopulos et al. Venous lengths are assumed equal to the arteries at the same anatomical level. Generic values for vessel lengths were taken as Leguy [72] showed that the mean flow is not sensitive to uncertainties in the lengths of the vessels and for AVF creation mean flow is the most important outcome. However, note that for other output parameters like the transit time of the waveforms this does not hold. In that case, the vessel lengths should be determined patient-specifically.

The wall thickness to radius ratio in the subclavian, axillary and brachial artery were set to 0.15 , in the ulnar, interosseus and radial artery a ratio of 0.20 was taken $[24,95,96]$. For all other arteries data from Stergiopulos [25] were used (Table 3.3). For veins a ratio of 0.10 was assumed. The anastomosis angle is unknown prior to surgery but has influences the pressure drop over the anastomosis. It was therefore varied between 30 and 60 degrees. 
Table 3.2: All patient-specific data that is measured within the clinic for each individual patient.

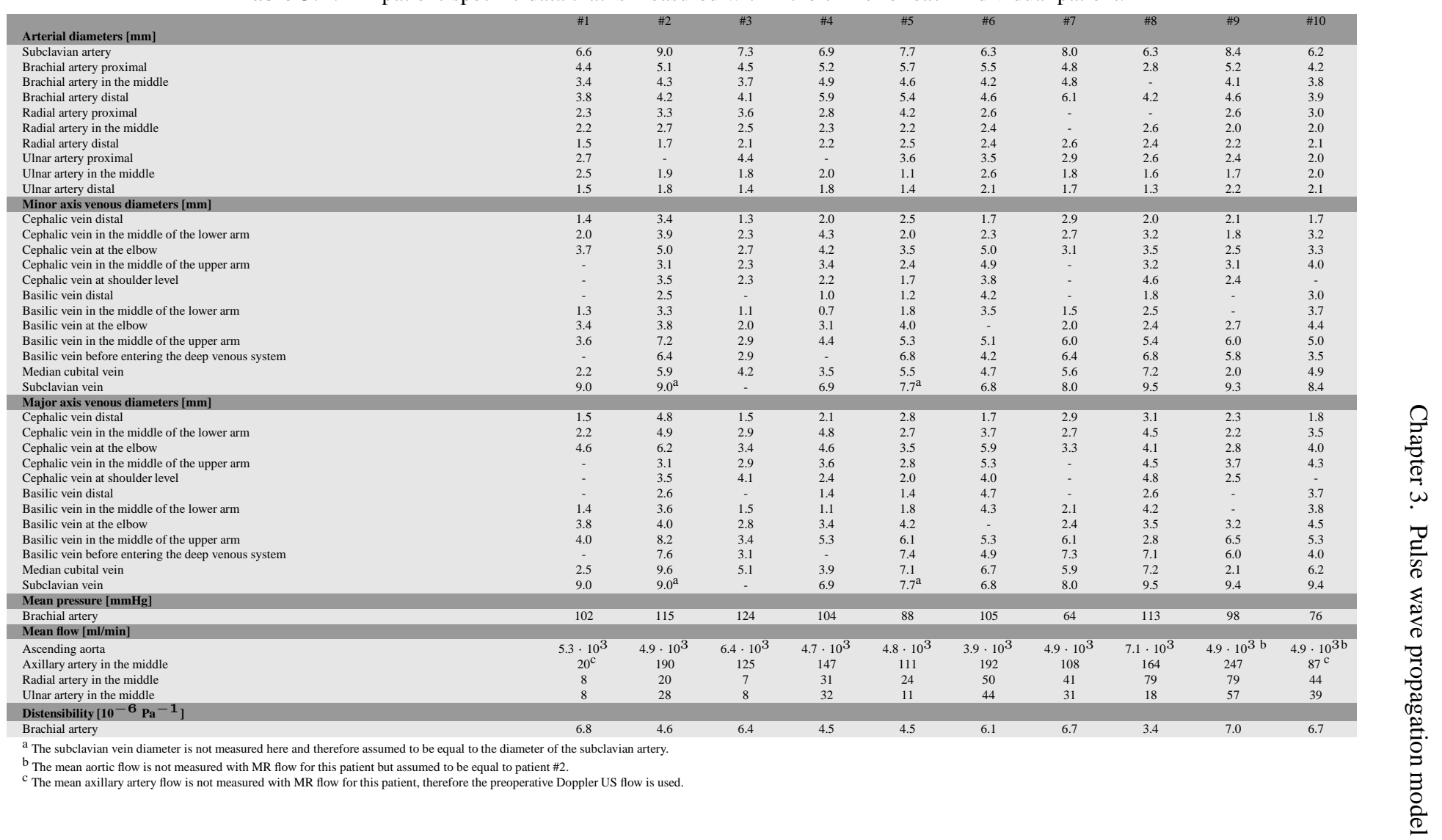


Table 3.3: Physiological and geometrical data of the arterial tree based on Stergiopulos et al. [25]. This table contains the segment length $l$, the proximal radius $a_{p}$, the distal radius $a_{d}$, the wall thickness $h$ and the elastic Young's modulus $E$.

\begin{tabular}{|c|c|c|c|c|c|c|}
\hline Edge nr. & Arterial edge name & $l\left[10^{-2} \mathrm{~m}\right]$ & $a_{p}\left[10^{-2} \mathrm{~m}\right]$ & $a_{d}\left[10^{-2} \mathrm{~m}\right]$ & $h\left[10^{-3} \mathbf{m}\right]$ & $E\left[10^{6} \mathrm{~Pa}\right]$ \\
\hline 1 & ascending aorta & 4.00 & 1.47 & 1.44 & 1.63 & 0.40 \\
\hline 2 & aortic arch A & 1.00 & 1.12 & 1.12 & 1.26 & 0.40 \\
\hline 3 & aortic arch A & 1.00 & 1.12 & 1.12 & 1.26 & 0.40 \\
\hline 4 & innominate & 3.40 & 0.62 & 0.62 & 0.80 & 0.40 \\
\hline 5 & aortic arch B + thoracic aorta & 9.10 & 1.07 & 0.999 & 1.13 & 0.40 \\
\hline 6 & left carotid & 4.10 & 0.370 & 0.370 & 0.63 & 0.40 \\
\hline 7 & left subclavian A & 3.40 & 0.423 & 0.423 & 0.66 & 0.40 \\
\hline 8 & left vertebral & 4.90 & 0.188 & 0.186 & 0.45 & 0.80 \\
\hline 9 & left subclavian B + axillary + brachial & 44.3 & 0.403 & 0.236 & 0.67 & 0.40 \\
\hline 10 & $\begin{array}{l}\text { left radial } \\
\text {. }\end{array}$ & 23.5 & 0.174 & 0.142 & 0.43 & 0.80 \\
\hline 11 & left ulnar proximal & 6.70 & 0.215 & 0.215 & 0.46 & 0.80 \\
\hline 12 & left interosseus & 7.90 & 0.091 & 0.091 & 0.28 & 1.60 \\
\hline 13 & left ulnar distal 1 & 5.70 & 0.203 & 0.188 & 0.46 & 0.80 \\
\hline
\end{tabular}

Elastic properties. For each patient the distension (change in diameter $d$ ) of the brachial artery was measured with ultrasound (Picus with ART.LAB functionality, ESAOTE B.V. Europe). Simultaneously, the brachial pressure $p_{b}$ was assessed from real-time noninvasive finger pressure measurements (NexFin, BMEYE B.V., The Netherlands). From the distension $\Delta d$ and the brachial pulse pressure $\Delta p_{b}$ the distensibility $D$ can be assessed from

$$
D \approx \frac{1}{\pi a_{0}^{2}} \frac{\Delta A}{\Delta p_{b}} \approx \frac{a_{s y s}^{2}-a_{d i a}^{2}}{a_{0}^{2} \Delta p_{b}}
$$

in which $a_{\text {sys }}$ and $a_{\text {dia }}$ are the systolic and diastolic radii. The determined distensibilities are given in Table 3.2.

By assuming a thick-walled tube formulation and a wall thickness to radius ratio of 0.15 , the Young's modulus of the brachial artery was determined from (3.11). This estimate for the (patient-specific) Young's modulus was also taken for the subclavian, axillary, ulnar, radial and interosseus artery. The Young's modulus for veins was assumed to be $1.2 \cdot 10^{5}$ $\mathrm{Pa}$ and derived from [97] by applying (3.12) and a wall thickness to radius ratio of 0.10 .

Constants. In all simulations blood density, blood viscosity, and the Poisson ratio were chosen to be $1.05 \cdot 10^{3} \mathrm{~kg} / \mathrm{m}^{3}, 3 \cdot 10^{-3} \mathrm{~Pa} \cdot \mathrm{s}$, and 0.5 [-] respectively [36].

\section{Boundary conditions}

Prescribed flows. As input the aortic flow waveform is used that is preoperatively determined by means of phase-contrast PC-MRI for each patient. In Table 3.2 the timeaveraged aortic flow is given, whereas in the Figures 3.5 and 3.6 an example of a patient- 
specific aortic flow waveform is presented.

Prescribed pressures. The extravascular pressure $p_{e}$ was assumed to be $0 \mathrm{mmHg}$. The venous branch that represents the AVF's draining vein was closed with a venous pressure $p_{v}$ of $10 \mathrm{mmHg}$ whereas the windkessels were closed with a venous pressure $p_{v, w k}$ of 0 $\mathrm{mmHg}$.

Windkessel parameters and distributed flow to small side-branches. The time constant $\tau$ was set to 1.1 seconds according to data from Leguy [72]. The peripheral resistance of an end-segment, $R_{p}$, was determined by (3.23). The mean arterial pressure was assessed from finger pressure measurement (Table 3.2). Flow waveforms through the axillary, ulnar and radial artery were determined by PC-MRI and the time-averaged flows were used to calculate peripheral resistance. By assuming that the interosseus artery and ulnar artery are subjected to equal Poiseuille wall shear stress (Murray's law [98]), the mean flow through the interosseus artery $\bar{q}_{i}$ can be determined by

$$
\bar{q}_{i}=\left(\frac{a_{0, i}}{a_{0, u}}\right)^{3} \bar{q}_{u}
$$

in which $\bar{q}_{u}$ is the mean ulnar artery flow, $a_{0, i}$ the interosseus diameter and $a_{0, u}$ the distal ulnar diameter. Furthermore, it was assumed that $0.5 \%$ of the cardiac output flows through the end-segment of the vertebral artery, while 7\% flows to the left and $7 \%$ to the right carotid artery, and that preoperatively, the flow to both arms is the same. The remaining cardiac output flows through the thoracic aorta. Blood flow through small side branches that were not modeled separately, was only applied in the axillary and brachial artery and represents the blood that flows to the muscles in the upper extremity. This amount of flow distributed for each patient was assessed from the difference between the time-averaged axillary artery flow and the sum of the time-averaged radial, ulnar and interosseus flows as determined by PC-MRI and (3.26).

Numerical constants. In all simulations the time step $\Delta t$ was set at $5 \mathrm{~ms}$ and the convergence criterium $\epsilon=10^{-5} \mathrm{~m}^{3} / \mathrm{s}$ was used. The simulations were stopped if the relative difference of mean pressures between two consecutive cardiac cycles was smaller than $10^{-5}$. 


\section{Simulations and analysis}

Before application of the lumped parameter implementation to patient-specific vascular access planning, we benchmarked our lumped parameter implementation with the spectral element implementation of Bessems [61] on a generic arterial tree.

The ten patient-specific lumped parameter pulse wave propagation models are used to simulate the effect of VA creation on the hemodynamics in the arm. First, pressure and flow waveforms in the brachial and distal radial artery are compared for both an upper and lower arm AVF creation to study the ability of the model to delineate flow increases and pressure changes resulting from an upper and a lower arm AVF creation.

To demonstrate how a patient-specific lumped parameter pulse wave propagation model might be able to support in the decision making of the AVF location, the direct postoperative flow for the lower arm AVF are compared to a threshold of $400 \mathrm{ml} / \mathrm{min}$; when a flow larger than $400 \mathrm{ml} / \mathrm{min}$ is predicted for the lower arm AVF, a lower arm AVF is suggested, otherwise an upper arm AVF is indicated. The suggested AVF configuration is compared to the configuration that is independently selected and created by a vascular surgeon with ample experience and expertise in creating VA for hemodialysis (more than 1000 AVFs created).

Finally, simulated mean brachial artery flow for the established AVF configuration is compared with the direct (within one week) postoperative mean brachial artery flow determined by duplex ultrasound. In our vascular lab, this mean brachial artery flow is determined by measuring the time-averaged maximum blood flow velocity with Doppler ultrasound and multiplying this with the cross-sectional area depicted from B-mode imaging, thus assuming a flat velocity profile. However, in reality the velocity profile will differ from a flat profile. The actual velocity profile within the brachial artery can be calculated from Womersley theory [84] in the case of fully developed flow. However, the high mean Reynolds numbers postoperatively $(\mathrm{O}(600-2000))$ will result in large entrance lengths $(\mathrm{O}(20-70 \mathrm{~cm})$ for a diameter of approximately $6 \mathrm{~mm})$ for the steady flow component and therefore the flow is not necessarily fully developed. In addition, the peak Reynolds numbers might even be higher $(\mathrm{O}(1500-6500))$ resulting in possible turbulent flow and thus more flat velocity profiles. For these reasons and, in addition, due to curvature, the real velocity profile is not exactly known. However, it is likely that the actual velocity profile is between a parabolic and a flat velocity profile. Therefore, we decided to correct for the velocity profile by a factor 0.75 and to define a range with as upper limit the flow determined with a flat profile and as lower limit the flow determined from a parabolic profile (corrected by 0.5 ). 


\subsection{Results}

No differences were found between the lumped parameter implementation in this study and the spectral element implementation of Bessems [61], demonstrating a correct realization of the proposed scheme.

\subsubsection{Vascular access planning}

\section{Preoperative results}
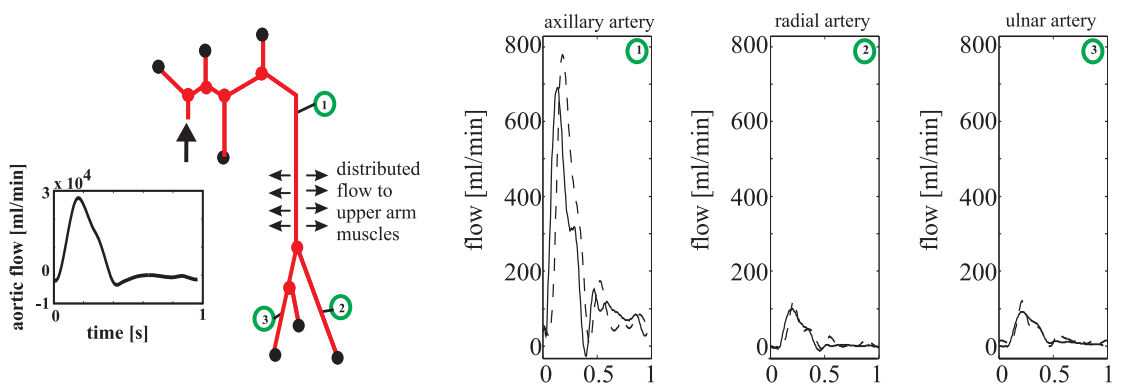

Figure 3.5: The predicted (solid) and MR measured (dashed) axillary, radial and ulnar artery flows as function of time for patient \#2.

The model was adapted to mimic the patient-specific pressure and flow waveforms preoperatively. Figure 3.5 shows simulated and measured (with MR) preoperative flow waveforms in the axillary, radial and ulnar artery for patient \#2 as an example. This patient is chosen as representative for all patients. Results for the other patients were equally good, however, in two patients (\#1 and \#10) measurement of the axillary artery flow was missing. Preoperatively, the simulated mean flows are almost equal (i.e. $0.18 \mathrm{l} / \mathrm{min}$ versus $0.19 \mathrm{l} / \mathrm{min}$ for the axillary artery flow, $0.019 \mathrm{l} / \mathrm{min}$ versus $0.020 \mathrm{l} / \mathrm{min}$ for the radial artery flow and $0.025 \mathrm{l} / \mathrm{min}$ versus $0.028 \mathrm{l} / \mathrm{min}$ for the ulnar artery flow) to the measured mean flows. This is expected, since these data are used as input for the model. However, simulated and measured flow waveforms in the axillary, ulnar and radial artery are also quite similar, although the simulated artery flow waveforms are slightly more attenuated than the measured ones. In addition, there is a small time delay ( $25 \mathrm{~ms})$ between the simulated and measured axillary artery flow, and reflections differ between the flow waveforms. This probably results from generic geometrical properties used as model input such as arterial lengths that influence wave propagation. The simulated mean arterial 
pressure of $112 \mathrm{mmHg}$ is close to the measured arterial pressure of $115 \mathrm{mmHg}$, which is also expected as it is used as model input. For all patients differences between simulated and measured mean pressure and flows were smaller than $5 \%$.

\section{Postoperative results: general effects}
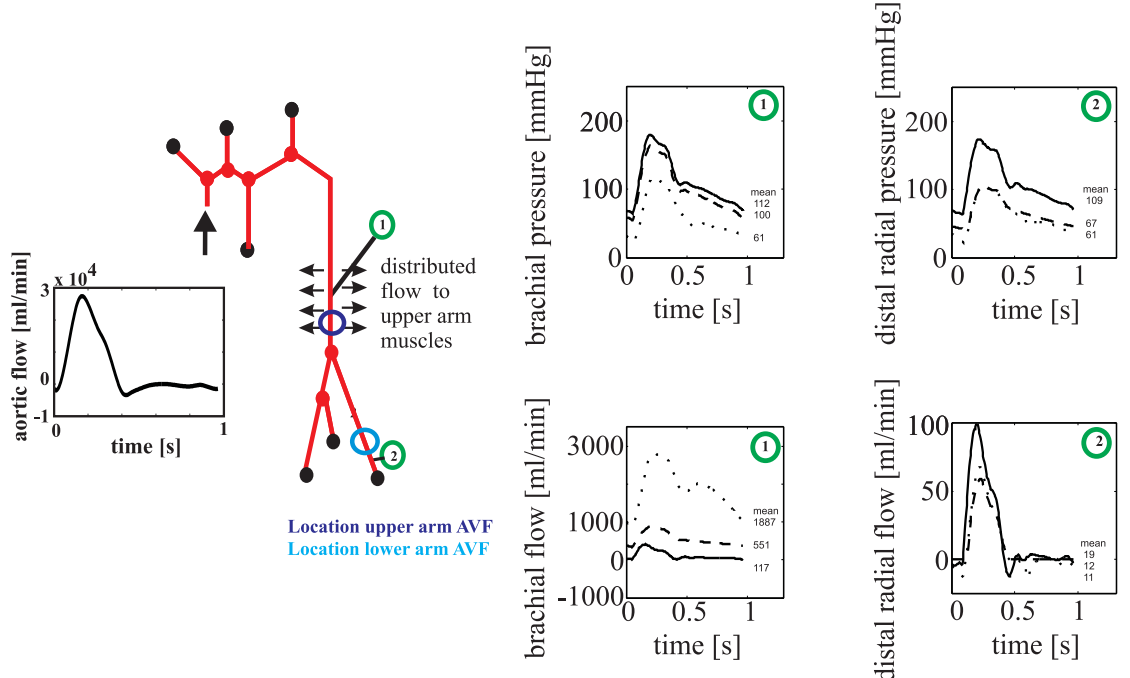

Figure 3.6: The pressure and flow waveforms calculated by the model in the brachial artery and distal radial artery before surgery (solid), after the creation of a lower arm AVF (dashed) and after creation of an upper arm AVF (dotted) for patient \#2. The anastomosis angle was set at 45 degrees.

Changes in pressure and flow after AVF creation are discussed by again using the simulation results of patient \#2. For all other patients, similar effects are observed. For this patient, the mean brachial artery flow increases from $0.12 \mathrm{l} / \mathrm{min}$ to $0.55 \mathrm{l} / \mathrm{min}$ after creation of a lower arm AVF (Figure 3.6) and to 1.9 1/min after creation of an upper arm AVF (brachio-basilic(BB) AVF). The anastomosis angle was set to 45 degrees for these simulations. The negative peak (backflow), that is present in the brachial artery flow waveform preoperatively, disappears after creation of both a lower and upper arm AVF. The mean brachial pressure decreases more after creation of the upper arm AVF (from 112 to 61 $\mathrm{mmHg}$ ) than after creation of the lower arm AVF (from 112 to $100 \mathrm{mmHg}$ ). The systolic and diastolic pressure also decrease postoperatively.

In the distal radial artery the mean flow decreases from $0.019 \mathrm{l} / \mathrm{min}$ to $0.011 \mathrm{l} / \mathrm{min}$ after creation of the lower arm AVF, whereas after creation of the upper arm AVF the radial 
artery flow decreases to $0.011 \mathrm{l} / \mathrm{min}$. The mean radial pressure decreases from $109 \mathrm{mmHg}$ to $66 \mathrm{mmHg}$ and $61 \mathrm{mmHg}$ after creation of the lower and upper arm AVF, respectively. In the distal radial artery a backflow is observed in the diastolic phase both preoperatively and after creation of an upper arm AVF, whereas backflow disappears after the creation of a lower arm AVF. In Figure 3.7 the effect of the anastomosis model and the anastomosis
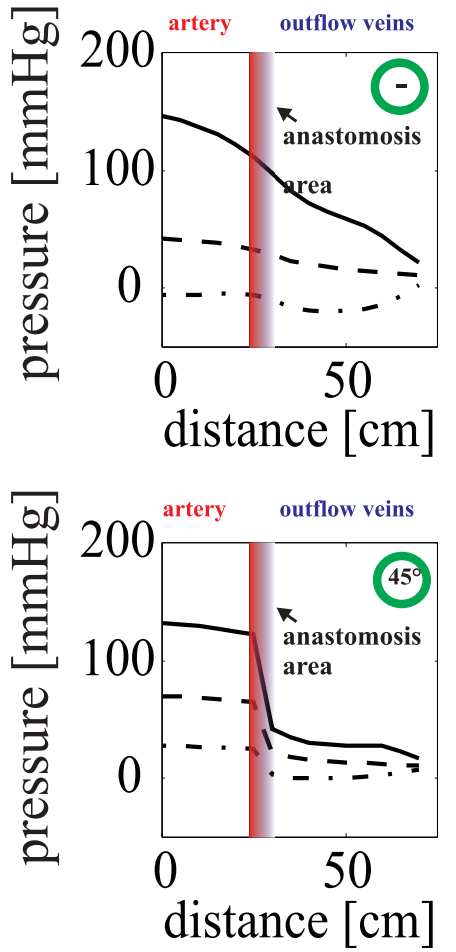
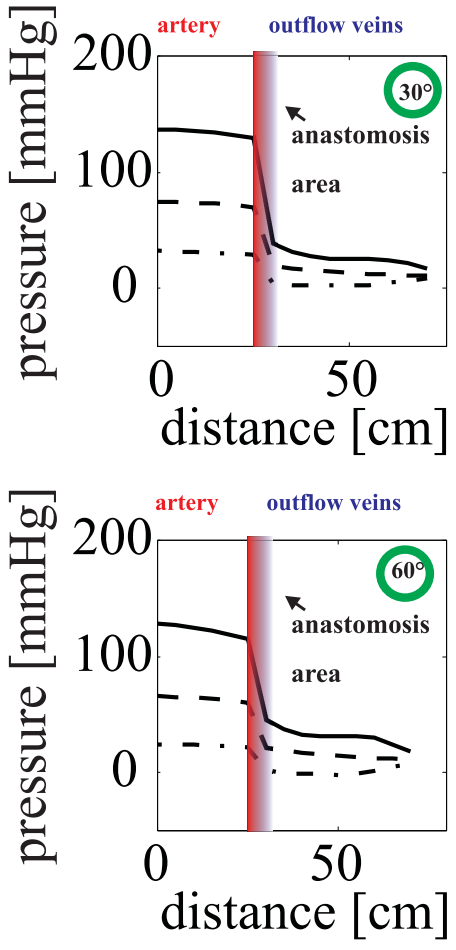

Figure 3.7: The pressure drop over the anastomosis from the axillary artery to the subclavian vein if no anastomosis element is incorporated (-) and with anastomosis element for an anastomosis angle of 30, 45 an 60 degrees. The systolic (solid), mean (dashed) and diastolic (dashed-dot) pressures are presented for patient $\# 2$ for the upper arm configuration (BB).

angle is shown for an upper arm AVF configuration (BBAVF) for patient \#2. Neglecting the extra pressure drop over the anastomosis results in a very low mean arterial pressure (approximately $43 \mathrm{mmHg}$ ) after AVF creation and a very high flow (3.1 1/min). Incorporating the anastomosis model results in a more realistic AVF flow whereas pulsatility distally from the anastomosis decreases. Furthermore, it can be observed that an acute 
anastomosis angle gives a larger pressure drop than a wider anastomosis angle. The negative diastolic venous pressure, observed in Figure 3.7, results from neglecting venous collapsibility.

\section{Postoperative results: surgical planning}

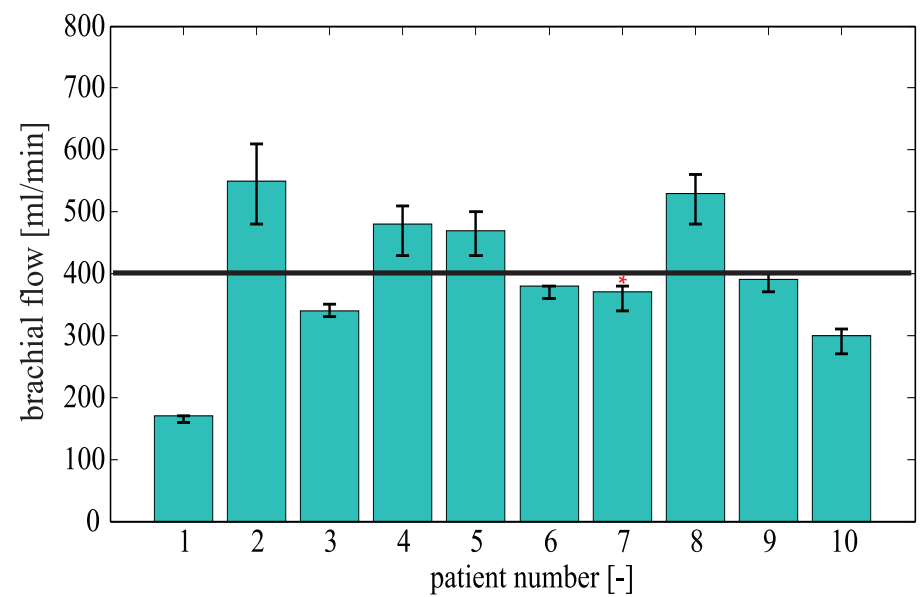

\begin{tabular}{|l|cccccccccc|}
\hline Patient number & $\mathbf{1}$ & $\mathbf{2}$ & $\mathbf{3}$ & $\mathbf{4}$ & $\mathbf{5}$ & $\mathbf{6}$ & $\mathbf{7}$ & $\mathbf{8}$ & $\mathbf{9}$ & $\mathbf{1 0}$ \\
& & & & & & & & & & \\
Simulation & $\mathrm{U}$ & $\mathrm{L}$ & $\mathrm{U}$ & $\mathrm{L}$ & $\mathrm{L}$ & $\mathrm{U}$ & $\mathrm{U}^{*}$ & $\mathrm{~L}$ & $\mathrm{U}$ & $\mathrm{U}$ \\
Surgeon & $\mathrm{U}$ & $\mathrm{L}$ & $\mathrm{U}$ & $\mathrm{L}$ & $\mathrm{L}$ & $\mathrm{U}$ & $\mathrm{L}$ & $\mathrm{L}$ & $\mathrm{U}$ & $\mathrm{U}$ \\
\hline
\end{tabular}

Figure 3.8: The calculated brachial artery flows after the creation of a lower arm AVF for all patients. The values are presented for an anastomosis angle of 45 degrees and the error bars represent the variation in flow prediction due to a variation in anastomosis angle ( \pm 15 degrees). The table shows the AVF configuration (U=upper arm, $\mathrm{L}=$ lower arm) based on the simulated postoperative flow and the AVF configuration selected by the surgeon. The superscript $*$ indicates a different choice.

In Figure 3.8 simulated brachial artery flow in case of a lower arm AVF is shown for all patients. In addition, the choice of the surgeon and the choice of the model are shown. The error bars on the simulated flows in this figure represent the influence of the anastomosis angle. With the current threshold of $400 \mathrm{ml} / \mathrm{min}$ in nine of the ten patients the surgeon and the model select the same AVF configuration (upper arm or lower arm AVF). The choice of the model and the surgeon differed in patient \#7. Clinically, the AVF of patient \#7 showed delayed maturation (three months) while all other AVFs matured within six weeks. 
Figure 3.9 shows that the absolute values of model predictions are in the same order of magnitude as the measured postoperative flows in six out of ten patients, whereas in the other four patients there is no overlap.

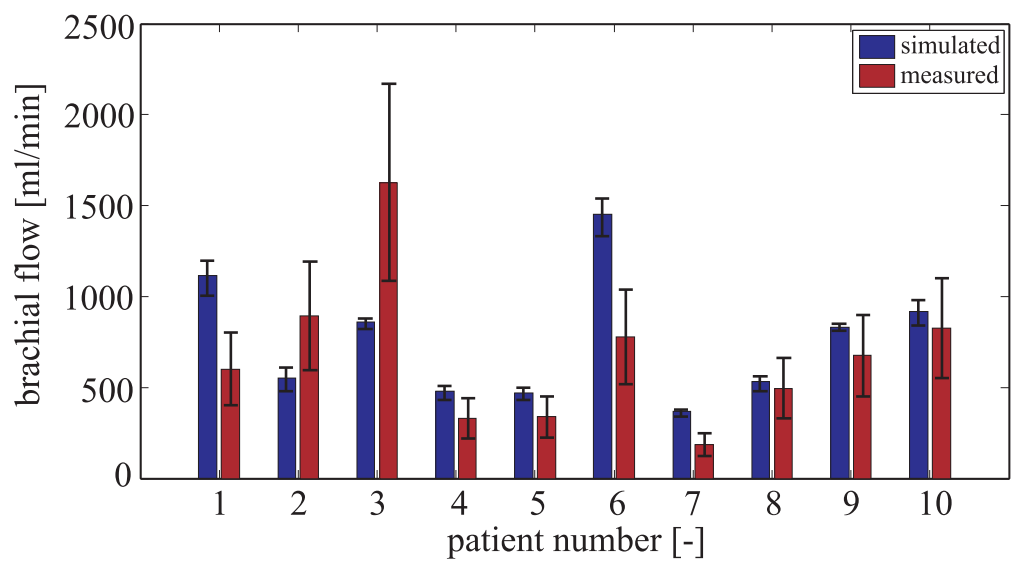

\begin{tabular}{|ccc|}
\hline Pat.\# & AVF type & Clinical remarks \\
1 & upper arm AVF (BB) & Fluid collection at anastomosis \\
2 & lower arm AVF (RC) & Young patient \\
3 & upper arm AVF (BC) & Significant side-branch \\
4 & lower arm AVF (RC) & - \\
5 & lower arm AVF (RC) & Arterial wall irregularities \\
6 & upper arm AVF (BC) & Small cannulation area \\
7 & lower arm AVF (RC) & Delayed maturation \\
8 & lower arm AVF (RC) & - \\
9 & upper arm AVF (BC) & - \\
10 & upper arm AVF (BC) & Cold hands/steal \\
\hline
\end{tabular}

Figure 3.9: The predicted brachial artery flows after the creation of the AVF configuration selected by the surgeon compared with the observed brachial artery flow for this AVF configuration directly after surgery (within 1 week). The error bars on the simulated flow represent the variation in flow prediction due to a variation in anastomosis angle ( \pm 15 degrees), whereas the error bars on the measured flow represent the flow based on a parabolic velocity profile and a flat profile. In the table (down) the patient characteristics are given. 


\subsection{Discussion}

This study aimed to derive and present a lumped parameter pulse wave propagation model that is robust and easily applicable in a clinical setting. Additionally, we showed how a patient-specific computational model, that predicts the postoperative flow, might support the surgeon in selecting an AVF configuration (i.e. upper or lower arm).

A lumped parameter pulse wave propagation model is presented with vascular segments based on an approximated velocity profile derived from boundary layer theory [61]. Pressure and flow waveforms calculated by the lumped parameter implementation are equal to the spectral element implementation of Bessems [61].

The lumped parameter pulse wave propagation model presented in this study is able to capture the pressure and flow changes after AVF creation. Both observed and simulated flow through the brachial artery increases significantly. Also a drop in blood pressure is observed after AVF creation. Additionally, the simulations show that the backflow normally present in the brachial artery disappears after AVF creation, as described in literature [99, 100]. The hemodynamical effects resulting from AVF creation were more pronounced in an upper arm AVF compared to a lower arm AVF, as is observed in clinical practice $[99,100]$.

To demonstrate how the lumped parameter pulse wave propagation model might be able to assist in VA surgery planning, the model is used to simulate AVF creation in ten ESRD patients. The model accurately describes preoperative hemodynamics: simulated mean pressures and flows differ by less than $5 \%$ from measured values and the simulated and measured flow waveforms are similar. Small differences in amplitude, transition time and shape due to reflections between measured and simulated flow waveforms can be explained from input inaccuracies for geometrical and mechanical properties of the vessels (for example by using generic values) inducing differences in wave propagation.

The lumped parameter pulse wave propagation model selects the same AVF configuration as the experienced surgeon in nine out of ten patients when using a threshold of $400 \mathrm{ml} / \mathrm{min}$. In one of the patients (\#7) the model suggested another AVF configuration. However, in this patient the maturation process was delayed (three months instead of six weeks) and the predicted low flow was possibly indicative for this. The threshold we used to select the optimal AVF is based on literature [32]. An AVF flow larger than $600 \mathrm{ml} / \mathrm{min}$ and smaller than $30 \%$ of the cardiac output after six weeks of maturation is generally accepted as a proper AVF flow $[9,10,13,14]$. In a lower arm AVF the direct postoperative flow is on average $60 \%$ of the flow after maturation while in an upper arm AVF this is almost $80 \%$ [32]. To be on the safe side, in the simulations a threshold of $400 \mathrm{ml} / \mathrm{min}$ was 
used. However, changing this threshold will naturally result in different conclusions. To establish the correct threshold a larger study is required. The results of this preliminary study give us the possibility to further elucidate the power of the model in a larger population.

The predicted postoperative flows are in the same order of magnitude as the measured postoperative flows in six out of ten patients. In the other four patients the predicted and observed flows differ. In patient \#3, the large difference can be explained from the fact that a major venous side-branch was not incorporated in the model, although this sidebranch significantly influenced the resulting AVF flow.

However, a comparison between measured and simulated flows is hampered by the inaccuracy in the flow predictions resulting from measurement inaccuracies in the input that is currently unknown. Hence in a future study, we need to perform an uncertainty analysis to determine the influence of input inaccuracies on our flow predictions. Furthermore, a sensitivity analysis needs to be performed to help to identify the parameters for which measurement improvements are necessary and most rewarding to further decrease the inaccuracy on the flow prediction, while less important parameters can be fixed and based on literature. The latter will result in parameter reduction and eases the adaptation of the model to patient-specific conditions and implementation in clinical practice potentially also. The comparison between measurement and simulations is also hampered by the accuracy of the measured mean brachial artery flow that is not exactly known. Next to the assumption of the velocity profile, is the uncertainty of Doppler flow measurements caused by measurements errors, like for example diameter measurement inaccuracy or an incorrect angle of the Doppler measurement. Therefore, in future research the inaccuracy of Doppler flow measurements should be determined and a method should be developed that is able to accurately measure the postoperative mean brachial artery flow, which will result in model and measurement comparisons that are more quantitatively.

In this study, we used a lumped parameter wave propagation model to predict the postoperative flows. Because the main output of interest was the mean brachial flow and the momentum and continuity equations were linearized, the wave propagation model can eventually be simplified to a model consisting of resistances only if a proper estimate for the anastomosis resistance is known. However, the anastomosis resistance is dependent on the flow and we therefore need at least locally a proper estimate of the flow waveform. In addition, to be able to incorporate, in future research, the nonlinear relation between the venous pressure and its cross-sectional area and adaptation laws, a wave propagation model is better suitable than a model consisting of resistances only. Especially, because the distributed locations were stenosis might develop can be of clinical interest. 
In future research, the pressure-flow relation for the anastomosis that was assumed to be similar to a T-junction should be validated, for example by 3D CFD simulations of anastomoses. An extra pressure drop due to unsteadiness in flow should possibly be included. Incorporating these model improvements will result in less uncertainty in the model description and therefore better flow predictions, however, it will also increase the model complexity and number of model input parameters, hereby increasing the effect of data uncertainty. Increasing the model complexity will thus not necessarily result in better flow predictions. Therefore, future research will first focus on the current model and applying this for prediction of flow including the reliability for a larger patient population.

\subsection{Conclusion}

In this study, we have presented a lumped parameter pulse wave propagation model that is easily implementable, robust, intuitive and comprehensible towards VA planning. Additionally, we showed that a patient-specific model can be used to simulate the hemodynamical effects after AVF creation and might assist in VA surgery planning. The advantage of such a model is that it allows for preoperative patient-specific simulations of multiple factors (e.g. diameters, accessory veins and the anastomosis) and their mutual influence on postoperative flow.

\section{Appendix: Governing equations, electrical analog and nu- merical scheme}

\section{Governing equations}

\section{Basic equations}

Here we will derive the 1D wave propagation equations by using the 1D Reynolds transport theorem. This part is based on Hughes and Lubliner [59] and van de Vosse and Stergiopulos et al. [55] .

\section{D Reynolds transport theorem}

In accordance with the work of Hughes and Lubliner [59], we take the geometry as depicted in Figure 3.10 as point of departure. The time-dependent volume $V(t)$ is bounded 


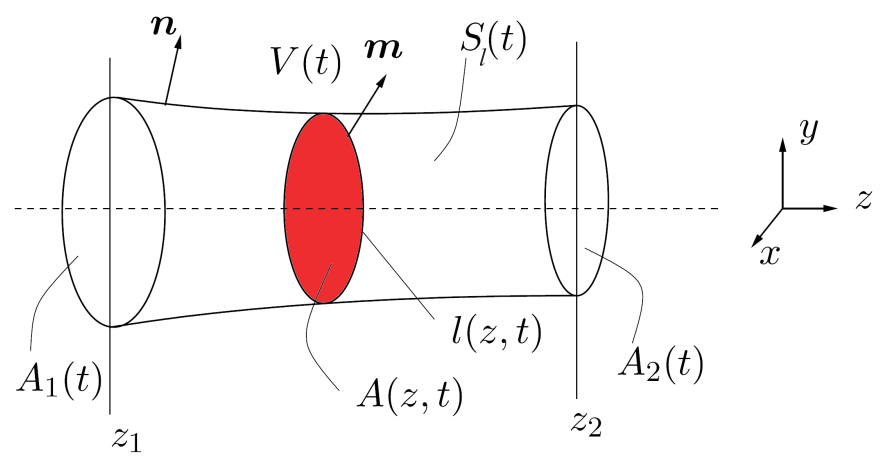

Figure 3.10: A vascular segment on which Reynolds transport theorem is applied.

by the total surface $S_{t}(t)$ that consists of two longitudinal surfaces $A_{1}$ and $A_{2}$ perpendicular to the z-axis at fixed locations $z_{1}$ and $z_{2}$ respectively, and the lateral surface of $V(t)$ denoted by $S_{l}$ and allowed to move with velocity $\mathbf{u}$. The outward normal to the surfaces $A_{1}, A_{2}$ and $S_{l}$ is given by $\mathbf{n}$. The Reynolds transport theorem can now be written as

$$
\frac{d}{d t}\left(\int_{V(t)} \psi d V\right)=\int_{V(t)} \frac{\partial \psi}{\partial t} d V+\int_{S_{t}(t)}(\psi \mathbf{u} \cdot \mathbf{n}) d S_{t}
$$

in which $\psi=\psi(\mathbf{x}, t)$ is an arbitrary function dependent on location and time and in which $d V$ and $d S_{t}$ are infinitesimal volume and surface elements respectively. We know define the luminal area mean $\bar{\psi}$ by

$$
\bar{\psi}=\frac{1}{A} \int_{A(z, t)} \psi d A .
$$

Knowing that $z_{1}$ and $z_{2}$ are fixed and independent on time, the left hand side of (3.27) can be written as

$$
\begin{aligned}
\frac{d}{d t}\left(\int_{V(t)} \psi d V\right)=\frac{d}{d t}\left(\int_{z_{1}}^{z_{2}}\left(\int_{A(z, t)} \psi d A\right) d z\right)=\frac{d}{d t} \int_{z_{1}}^{z_{2}} A \bar{\psi} d z & \\
= & \int_{z_{1}}^{z_{2}} \frac{\partial}{\partial t}(A \bar{\psi}) d z
\end{aligned}
$$

Now we introduce the relative velocity of the lateral vessel wall $\mathbf{w}=\mathbf{u}-\mathbf{v}$ and the assumptions that $\mathbf{u}=\mathbf{0}$ at the longitudinal surfaces $\left(A_{1}\right.$ and $\left.A_{2}\right)$ and that the flow in incompressible (i.e. $\nabla \cdot \mathbf{v}=0$ ). The second term on the right hand side in (3.27) results 
in

$$
\int_{S_{t}(t)}(\psi \mathbf{u} \cdot \mathbf{n}) d S_{t}=\int_{S_{l}}(\psi \mathbf{w} \cdot \mathbf{n}) d S_{l}+\int_{V(t)}(\mathbf{v} \cdot \nabla \psi) d V-\int_{z_{1}}^{z_{2}} \frac{\partial}{\partial z}\left(A \overline{\psi v_{z}}\right) d z
$$

after application of the Gauss divergence theorem [59]. Substituting (3.29) and (3.30) in (3.27) results in

$$
\int_{z_{1}}^{z_{2}}\left(\frac{\partial}{\partial t}(A \bar{\psi})+\frac{\partial}{\partial z}\left(A \overline{\psi v_{z}}\right)\right) d z=\int_{V(t)}\left(\frac{\partial \psi}{\partial t}+\mathbf{v} \cdot \nabla \psi\right) d V+\int_{S_{l}}(\psi \mathbf{w} \cdot \mathbf{n}) d S_{l}
$$

or equivalently:

$$
\begin{aligned}
\int_{z_{1}}^{z_{2}}\left(\frac{\partial}{\partial t}(A \bar{\psi})+\frac{\partial}{\partial z}\left(A \overline{\psi v_{z}}\right)\right) d z=\int_{z_{1}}^{z_{2}}\left(\int _ { A } \left(\frac{\partial \psi}{\partial t}\right.\right. & +\mathbf{v} \cdot \nabla \psi) d A) d z \\
& +\int_{z_{1}}^{z_{2}}\left(\oint_{l} \psi \mathbf{w} \cdot \mathbf{n} d l\right) d z
\end{aligned}
$$

with $d l$ an infinitesimal line element on the closed curve $l$ bounding $A$. Finally, the local form of the Reynolds transport theorem with respect to the axial coordinate $z$ is given by

$$
\frac{\partial}{\partial t}(A \bar{\psi})+\frac{\partial}{\partial z}\left(A \overline{\psi v_{z}}\right)=\int_{A(z, t)}\left(\frac{\partial \psi}{\partial t}+\mathbf{v} \cdot \nabla \psi\right) d A+\oint_{l(z, t)} \psi w_{n} d l
$$

with $w_{n}=\mathbf{w} \cdot \mathbf{n}$ is the normal component of $\mathbf{w}$ on curve $l$. Equation (3.33) is used as basis for the derivation of the mass and momentum equations.

\section{D Mass conservation equation}

By taking $\psi=1$ in the local Reynolds transport theorem (3.33) and by introducing the flow $q=A \bar{v}_{z}$, we arrive at the integrated continuity equation in $(A, q)$ :

$$
\frac{\partial A}{\partial t}+\frac{\partial q}{\partial z}+\Psi=0
$$

with $\Psi=-\oint_{l(z, t)} w_{n} d l$ the volumetric outflow per unit length. Substitution of the constitutive relation, expressing the response of the vessel wall to pressure variations, according to

$$
\frac{\partial A}{\partial t}=\frac{\partial A}{\partial p} \frac{\partial p}{\partial t} \equiv C \frac{\partial p}{\partial t}
$$


results in the continuity equation in $(p, q)$-formulation:

$$
C \frac{\partial p}{\partial t}+\frac{\partial q}{\partial z}+\Psi=0
$$

with $C=C(z, t)$ the area compliance of the vessel that can be obtained from experimental data or from a solid mechanical model of the arterial wall.

\section{$1 D$ momentum equation}

The momentum equation for the fluid inside volume $V(t)$ in three dimensions is given by

$$
\rho\left(\frac{\partial \mathbf{v}}{\partial t}+\mathbf{v} \cdot \nabla \mathbf{v}\right)=\rho \mathbf{f}+\nabla \cdot \tau
$$

with $\rho$ blood density, $\mathbf{f}$ the body forces and $\tau$ the Cauchy stress tensor. For (generalized) Newtonian fluids, the Cauchy tensor is given by

$$
\tau=-p \mathbf{I}+2 \eta \mathbf{D}
$$

with $\eta$ the dynamic blood viscosity and $\mathbf{D}$ the rate of deformation tensor. If we define the characteristic axial length scale $L_{z}$ to be the minimum of the wavelength $\lambda$ of the pressure and flow waves traveling through the arteries and if we associate the wavelength (or curvature) with axial changes in the cross-sectional area $\left(\frac{\partial A}{\partial z}\right)$, the following scaling is introduced for the coordinates: $\left(x^{\prime}, y^{\prime}\right)=\frac{(x, y)}{a_{0}}$ and $z^{\prime}=\frac{z}{L_{z}}$ with $a_{0}=\sqrt{\frac{A_{0}}{\pi}}$. The velocity can be scaled according to: $\left(v_{x}{ }^{\prime}, v_{y}^{\prime}\right)=\frac{\left(v_{x}, v_{y}\right)}{U}$ and $v_{z}^{\prime}=\frac{v_{z}}{V}$ with $U=\frac{a_{0}}{L_{z}} V$. If we now assume that all body forces are small (i.e. $f_{z} \ll \frac{U^{2}}{L_{z}}$ and $\max \left(f_{x}, f_{y}\right) \ll \frac{V^{2}}{a_{0}}$ ) or work mainly in the axial direction (i.e. $\left.f_{z} \gg\left(\frac{L_{z}}{a_{0}}\right)^{2} \max \left(f_{x}, f_{y}\right)\right)$, or in the case of long waves and gradual changes of cross-sectional area (i.e $\frac{L_{z}}{a_{0}} \gg 1$ ), we can neglect all terms that scale with $\frac{a_{0}}{L_{z}}$. The pressure $p$ is in that case constant over the cross-sectional area and only depends on $z$ and $t$. The 3D momentum equation in the $z$-direction will then reduce to

$$
\rho \frac{\partial v_{z}}{\partial t}+\rho\left(\mathbf{v}_{A} \cdot \nabla_{A} v_{z}\right)+\frac{\partial p}{\partial z}=\rho f_{z}+\nabla_{A} \cdot \tau_{A}
$$

with $\tau_{A}=\eta\left(\nabla_{A} \mathbf{v}_{A}+\left(\nabla_{A} \mathbf{v}_{A}\right)^{T}\right)$, with the in-plane velocity $\mathbf{v}_{A}=v_{x} \mathbf{e}_{x}+v_{y} \mathbf{e}_{y}$ and gradient operator $\nabla_{A}=\mathbf{e}_{x} \frac{\partial}{\partial x}+\mathbf{e}_{y} \frac{\partial}{\partial y}$. Integration of (3.39) over the cross-sectional area 
results in

$$
\rho \int_{A} \frac{\partial v_{z}}{\partial t} d A++\rho \int_{A}\left(\mathbf{v}_{A} \cdot \nabla_{A} v_{z}\right) d A+A \frac{\partial p}{\partial z}=A \rho f_{z}+\int_{A}\left(\nabla_{A} \cdot \tau_{A}\right) d A .
$$

By using the divergence theorem and after dividing by $\rho$ we obtain for a constant blood viscosity

$$
\int_{A} \frac{\partial v_{z}}{\partial t} d A+\int_{A}\left(\mathbf{v}_{A} \cdot \nabla_{A} v_{z}\right) d A+\frac{A}{\rho} \frac{\partial p}{\partial z}=A f_{z}+\oint_{l}\left(\frac{\eta}{\rho} \nabla_{A} v_{z} \cdot \mathbf{m}\right) d l
$$

with $\mathbf{m}=\left(m_{x}, m_{y}, 0\right)$ the outerward normal to $l$. The first term of (3.41) can now be rewritten by using the local Reynolds transport theorem (3.27) for $\psi=v_{z}$. The momentum equation in (3.41) now becomes

$$
\frac{\partial q}{\partial t}+\int_{A}\left(\mathbf{v}_{A} \cdot \nabla_{A} v_{z}\right) d A+\frac{\partial\left(A \overline{v_{z}^{2}}\right)}{\partial z}+\frac{A}{\rho} \frac{\partial p}{\partial z}=A f_{z}+\oint_{l}\left(\frac{\eta}{\rho} \nabla_{A} v_{z} \cdot \mathbf{m}+v_{z} w_{n}\right) d l
$$

For uniaxial flow $\int_{A}\left(\mathbf{v}_{A} \cdot \nabla_{A} v_{z}\right) d A=0$ because $\mathbf{v}_{A}=0$. Now we introduce the approximation $\oint_{l}\left(\frac{\eta}{\rho} \nabla_{A} v_{z} \cdot \mathbf{m}\right) d l \approx \frac{2 \pi a_{0}}{\rho} \tau_{w}$ in which $\tau_{w}$ is the wall shear stress defined here as $\tau_{w}=\left.\eta \frac{\partial v_{z}}{\partial r}\right|_{r=a_{0}}$ with $r$ the radial direction. After assuming longitudinal tethering of the tube, we are allowed to take $v_{z}=0$ at the vessel wall and thus $\oint_{l} v_{z} w_{n} d l=0$. Note further that $\frac{\partial\left(A \overline{v_{z}^{2}}\right)}{\partial z}=\frac{\partial}{\partial z}\left(\int_{A} v_{z}^{2} d A\right)$. By using the properties and assumptions above, the momentum equation results in

$$
\frac{\partial q}{\partial t}+\frac{\partial}{\partial z}\left(\int_{A} v_{z}^{2} d A\right)+\frac{A}{\rho} \frac{\partial p}{\partial z}=A f_{z}+\frac{2 \pi a_{0}}{\rho} \tau_{w} .
$$

To solve (3.43) with respect to $p$ and $q$ together with the 1D mass equation and a constitutive relation between $A$ and $p$, assumptions on the shape of the velocity profile as function of $p$ and $q$ have to be made to be able to make at least proper approximations of the convective term $\left(\int_{A} v_{z}^{2} d A\right)$ and the wall shear stress term. In the next subsection, the approximated velocity profile derived by Bessems et al. [61] and used in this study is described.

\section{Approximated velocity profile}

The approximated velocity profile to estimate the convective term and the wall shear stress in equation (3.43) is based on asymptotic solutions for the Stokes boundary layer in which viscous forces are dominant and the central core of the tube in which inertia forces are 
dominant, matched at the location of the Stokes boundary layer thickness from the wall. This approximated velocity profile as function of $p$ and $q$ in the time domain was derived by Bessems et al. [61]. The approximations for the wall shear stress and the convective term are

$$
\tau_{w} \approx-\frac{a_{0}}{2}\left[c_{q}(\alpha) R_{0} q-\left(c_{p}(\alpha)-1\right) \frac{\partial p}{\partial z}\right]
$$

and

$$
\int_{A} v_{z}^{2} d A \approx \delta_{1}(\alpha) \frac{q^{2}}{A}
$$

respectively. Parameters $\delta_{1}, c_{p}$ and $c_{q}$ solely dependent on the Womersley number ( $\alpha=$ $a_{0} \sqrt{\frac{\omega \rho}{\eta}}$ with $\omega$ angular frequency). The parameters $c_{p}$ and $c_{q}$ are given by [61]

$$
c_{p}=1+\frac{\sqrt{2}}{\alpha}\left(1-\frac{\sqrt{2}}{2 \alpha}\right), \quad c_{q}=\frac{\alpha}{4 \sqrt{2}}\left(1-\frac{\sqrt{2}}{2 \alpha}\right)^{-1} \quad \text { for } \quad \alpha>\sqrt{2}
$$

and

$$
c_{p}=\frac{3}{2}, \quad c_{q}=\frac{1}{2} \quad \text { for } \quad \alpha \leq \sqrt{2}
$$

Parameter $\delta_{1}$ is neglected in our study because the contribution of the convection is expected to be small in the arm vasculature $[65,87]$. However, for more details we refer to Bessems et al. [61].

The approximated velocity profile converges to parabolic and flat profiles for respectively low and high Womersley numbers $[61,86]$. Furthermore, the velocity profiles correspond fairly well with velocity profiles obtained from Womersley theory [61].

\section{Lumped segment: electrical analog}

Previously, we showed that the momentum equation (3.4) can be represented by a lumped parameter model (Figure 3.11, left panel) consisting of a parallel arrangement of a resistor per segment length $R_{1}$ and an inertance per unit length $L_{1}$ in series with a Poiseuille resistor per segment length $R_{0}$ [86]. This arrangement of the electrical elements is based on boundary layer theory with $L_{1}$ depicting the inertia dominated core and $R_{1}$ the viscous dominated boundary layer. Mathematical expressions of these parameters, $R_{1}$ and $L_{1}$, are 
given by [86]

$$
R_{1}=f(\alpha) R_{0} \quad \text { and } \quad L_{1}=g(\alpha) L_{0}
$$

with

$$
f(\alpha)=\left[\frac{c_{q}(\alpha)-\left(2-c_{p}(\alpha)\right)}{2-c_{p}(\alpha)}+\frac{\alpha^{4}}{64\left(c_{q}(\alpha)-\left(2-c_{p}(\alpha)\right)\right)\left(2-c_{p}(\alpha)\right)}\right],
$$

and

$$
g(\alpha)=\left[64 \frac{\left(c_{q}(\alpha)-\left(2-c_{p}(\alpha)\right)\right)^{2}}{\left(2-c_{p}(\alpha)\right) \alpha^{4}}+\frac{1}{2-c_{p}(\alpha)}\right] .
$$

It was also shown by Huberts et al. [86] that the relative impedance of this lumped parameter model corresponds to the relative impedance of Womersley theory [84]. Since $R_{1}$ and $L_{1}$ are frequency dependent (due to the Womersley number), they should be evaluated in the frequency domain. However, in this study we will need to include nonlinear
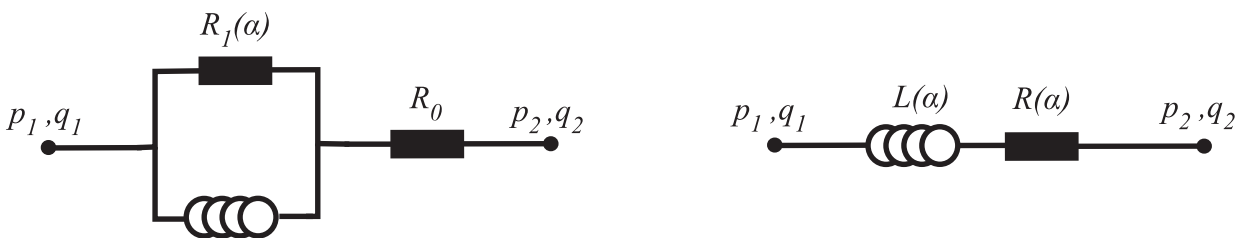

$L_{1}(\alpha)$

Figure 3.11: Different lumped parameter models that represent the momentum equation (3.4). The left lumped parameter model is based on boundary layer theory and derived in Huberts et al. [86]. The right lumped parameter model is used in the current study.

elements, e.g. for the anastomosis, and a frequency domain analysis is no longer possible. Here, we thus chose for an analysis in the time domain, analogous to Bessems et al. [61]. In time domain, we evaluate $R_{1}$ and $L_{1}$ only for the characteristic frequency of a heartbeat $\left(\omega_{0}\right)$, as it contains most of the signal's power. However, in that case, pressure and flow waveforms of the lumped parameter pulse wave propagation model consisting of segments represented by the lumped parameter element from Huberts et al. [86], are too much attenuated for higher frequencies $(>6 \mathrm{~Hz}$ ) compared to Womersley theory [84], resulting in deviations in pressure and flow waveforms. Therefore, in this study, we use a lumped parameter segment consisting of a Womersley number dependent resistor per unit length $R$ and Womersley number dependent inductor per unit length $L$ in series 
(Figure 3.11, right panel), which fits Womersley theory better than the parallel configuration, when evaluated only for the characteristic frequency. Parameters $L$ and $R$ stem from momentum equation (3.4) while $c_{p}$ and $c_{q}$ are evaluated for the characteristic frequency, which results in

$$
-\frac{\partial p}{\partial z}=R q+L \frac{\partial q}{\partial t}
$$

with

$$
R=\left[\frac{c_{q}\left(\alpha_{0}\right)}{2-c_{p}\left(\alpha_{0}\right)}\right] R_{0} \quad \text { and } \quad L=\left[\frac{1}{2-c_{p}\left(\alpha_{0}\right)}\right] L_{0} .
$$

Herein, $\alpha_{0}$ is the Womersley number corresponding to the characteristic frequency.

Note that when analyses are performed including all frequencies, both lumped parameter segments (i.e. (3.48) and (3.52) represented in Figure 3.11) give the same results.

\section{Matrix assembly and numerical scheme}

In this appendix we describe the assembly of the system matrix and the numerical scheme. In Wolters et al. an analogous derivation for the system matrix is presented [40]. The lumped parameter wave propagation model is described by a set of equations that are derived from the behavior of the constituting electrical elements, i.e. a resistor $R$, inductor $L$ and/or compliance $C$ within each segment (either an arterial, venous, anastomosis or windkessel segment). With $q^{e}=\left[q_{1}, q_{2}\right]^{T}$ the column of locally defined nodal flows and $\underline{\sim}^{e}=\left[p_{1}, p_{2}\right]^{T}$ the column of locally defined nodal pressures, the pressure-flow relation for the segment resistor $R$, the inductor $L$, and the compliance $C$ are given by, respectively,

$$
\underline{q}^{e}=\underline{R}_{e}^{r} \underline{\sim}^{p}, \quad \underline{q}^{e}=\underline{L}_{e}^{r} \int_{\tau=0}^{\tau=\tau} \underline{p}^{e}(\tau) d \tau+\underline{q}^{e}(0), \quad \underline{q}^{e}=\underline{C}_{e} \dot{\sim}_{\sim}^{e}
$$

with

$$
\underline{R}_{e}^{r}=\frac{1}{R}\left(\begin{array}{cc}
1 & -1 \\
-1 & 1
\end{array}\right) \quad, \quad \underline{L}_{e}^{r}=\frac{1}{L}\left(\begin{array}{cc}
1 & -1 \\
-1 & 1
\end{array}\right) \quad, \quad \underline{C}_{e}=C\left(\begin{array}{cc}
1 & -1 \\
-1 & 1
\end{array}\right)
$$

the electrical element matrices. Note that $R$ can represent $Z_{0}, R_{w k}, R(\alpha), R_{p}, R_{d}$ or $R_{L}$ depending on the modeled segment, whereas $C$ can represent both $C_{w k}$ in the case of a windkessel segment and $C_{0} / 2$ or $C_{0, v} / 2$ in the case of an arterial or venous segment 
respectively. The parameter $L$ is only representing $L(\alpha)$. By assembling the equations describing the pressure-flow relations for all electrical elements within the segments, by assuming an analogue of Kirchhoff's current law to satisfy element-to-element mass conservation and by using the property that coinciding local nodes have equal pressures, the assembling process results in the following set of integral-differential equations

$$
\underline{C} \underset{\sim}{\partial t}+\underline{R}^{r} \underset{\sim}{p}+\underline{L}^{r} \underline{h}^{n}+\underset{\sim}{q}(0)=\underline{q}_{e}
$$

with

$$
\underline{h}^{n}=\int_{\tau=0}^{\tau=t} \underset{\sim}{p}(\tau) d \tau
$$

The matrices $\underline{C}, \underline{R}^{r}$ and $\underline{L}^{r}$ thus contain all compliances, reciprocal resistances and reciprocal inertances respectively. $q_{e}$ is a column vector ( $\mathrm{N}$ by 1 ) that only contains nonzero values at nodes where flow is prescribed. $\underset{\sim}{p}=\left[p_{1}, p_{2}, \ldots, p_{N}\right]^{T}$ is a column vector ( $\mathrm{N}$ by 1) containing global nodal pressure values.

The time derivative $\left(\frac{\partial p}{\partial t}\right)$ and time integral $\left(\underline{h}^{n}\right)$ in the set of integral-differential equations (3.54) are approximated by using an (implicit) trapezoidal rule. The initial conditions are $\underline{q}(0)=0, \underset{\sim}{p}(0)=0$ and $\left.\frac{\partial p}{\partial t}\right|_{\tau=0}=0$. The final scheme is then given by

$$
\underline{K}_{\sim}^{p}=\underline{q}_{e}^{n}+{\underset{\sim}{f}}^{n-1}
$$

with

$$
\underline{K}=\frac{2}{\Delta t} \underline{C}+\underline{R}^{r}+\frac{\Delta t}{2} \underline{L}^{r}
$$

and

$$
{\underset{\sim}{f}}^{n-1}=\frac{2}{\Delta t} \underline{\sim}^{C p^{n-1}}+\underline{C}\left(\frac{\partial p}{\partial t}\right)^{n-1}-\underline{L}^{r} \underline{h}^{n-1}-\frac{\Delta t}{2} \underline{L}^{r}{\underset{\sim}{p}}^{n-1} .
$$

Parameter $\Delta t$ is the time step, and $n$ and $n-1$ refer to the current time step and one time step earlier.

By incorporating flow dependent resistances (3.17) and (3.18) for the anastomosis, the system becomes nonlinear and is given by

$$
\underline{C} \underset{\frac{\partial}{\partial t}}{\partial t} \underline{R}^{r}\left(q^{*}, A^{*}, \theta, q_{v}, q_{d}, q_{p}, A_{p}\right) \underset{\sim}{p}+\underline{L}^{r} \underline{h}^{n}=\underline{q}_{e} .
$$


To solve (3.59) an iterative procedure is used. In the first iteration step $i$ the system matrices of the linear equations are used to arrive at the first approximation $\underline{\sim}_{i}$. By using an analogue of Ohm's law the flows $q_{v_{i}}$ and $q_{d_{i}}$ through the resistances in (3.17) and (3.18), next to the proximal flow $q_{p_{i}}$ and $q^{*}$, are determined and used to update the system matrix to $\underline{R}_{i}{ }^{r}$. By inserting $\underline{p}_{i}$ and the updated matrix $\underline{R}_{i}{ }^{r}$ in (3.59) an approximation $q_{e_{i}}$ for $q_{e}$ is derived. The approximated $q_{e_{i}}$ is compared with the prescribed column vector

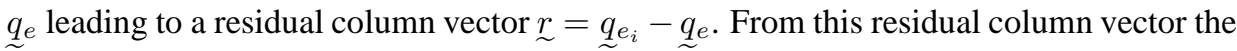
$\infty$-norm is calculated given by

$$
\|\underline{\mid r}\|_{\infty}=\max _{1 \leqslant k<N}\left|r_{k}\right| .
$$

If the norm is larger than a predefined convergence criterium $\epsilon$, the next iteration is executed, i.e. the approximation $\underline{p}_{i+1}$ is calculated with the updated matrix $\underline{R}_{i}^{r}$, until the convergence criterium is reached. A schematic overview of the numerical solving strategy is given in Figure 3.12. 


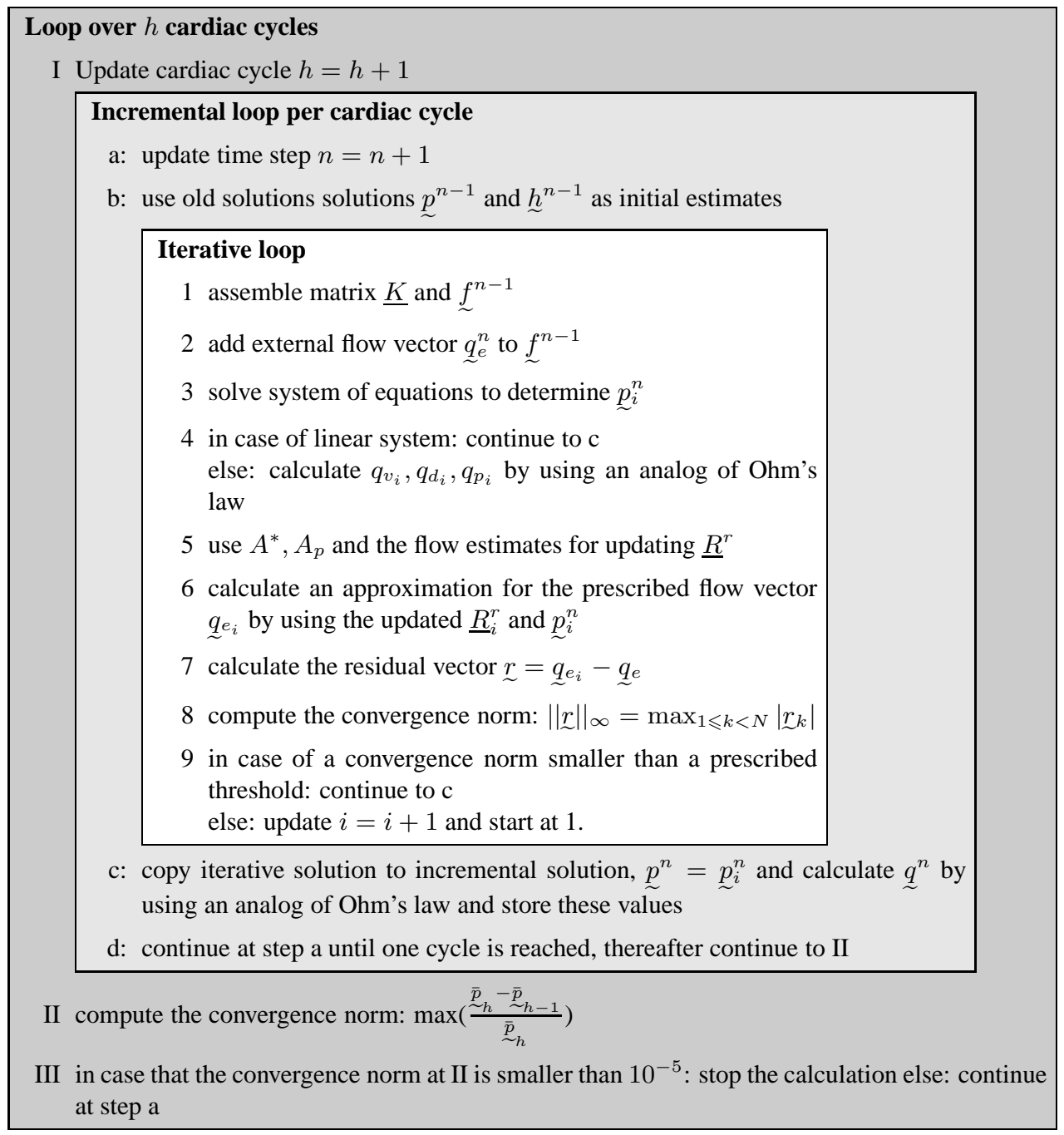

Figure 3.12: Schematic of the iterative scheme. 


\section{Chapter 4}

\section{A sensitivity analysis to improve the measurement protocol}

This Chapter is based on:

W. Huberts, C. de Jonge, W.P.M. van der Linden, M.A. Inda, J.H.M. Tordoir, F.N. van de Vosse, E.M.H. Bosboom, A sensitivity analysis to improve the measurement protocol to assess parameters for a personalized pulse wave propagation model: application to AVF surgery, Submitted, (2011) 


\begin{abstract}
Previously, a pulse wave propagation model was developed that has potential in supporting decisionmaking in vascular access surgery for hemodialysis. To adapt the wave propagation model to personalized conditions, patient-specific input parameters should be available. In clinics, the number of measurable input parameters is limited which results in sparse datasets. In addition, patient data are compromised with uncertainty. These uncertain and incomplete input datasets will result in model output uncertainties. By means of a sensitivity analysis the propagation of input uncertainties into output uncertainty can be studied which can give directions for input measurement improvement. In this study, a computational framework has been developed to perform such a sensitivity analysis with a variance-based method and Monte Carlo simulations. The framework was used to determine the influential parameters of our pulse wave propagation model applied to vascular access surgery, with respect to parameter prioritization and parameter fixing. With this we were able to determine the model parameters that have the largest influence on the predicted mean brachial flow and systolic radial artery pressure after vascular access surgery. Of all 73 parameters 51 could be fixed within their measurement uncertainty interval without significantly influencing the output, while 16 parameters importantly influence the output uncertainty. Measurement accuracy improvement should thus focus on these 16 influential parameters. The most rewarding are measurement improvements of the following parameters: the mean aortic flow, the aortic windkessel resistance, the parameters associated with the smallest arterial or venous diameters of the AVF in- and outflow tract and the radial artery windkessel compliance.
\end{abstract}




\subsection{Introduction}

A well-functioning vascular access is crucial for successful hemodialysis therapy. Such a vascular access is used to connect the patient's circulation to the artificial kidney and should provide the blood flow rate of $300 \mathrm{ml} / \mathrm{min}$ required for efficient treatment $[8,9]$. The best option for a vascular access is an arteriovenous fistula (AVF) in the arm, which is a surgically created permanent connection between an artery and vein $[8,9]$ resulting in a five- to thirtyfold flow increase and vessel dilation and remodeling. An AVF can be created at several positions in the arm and the optimal location is preoperatively selected by a vascular surgeon. Functioning lower arm AVFs have statistically better long-term patency rates and lower complication rates than upper arm AVFs [8, 9]. However, for a significant number of patients (up to 50\%), the resulting blood flow after creation of a lower arm AVF can be too low to enable hemodialysis $[6,7,10]$. Conversely, the blood flow in upper arm AVFs may be too high, leading to cardiac problems and/or distal hand ischemia in $20 \%$ of all upper arm AVFs $[6,7,8,12]$. In addition to the high blood flow, also a low systolic finger pressure after AVF creation is indicative for the development of distal ischemia $[7,8,9]$.

In previous work, a distributed lumped parameter pulse wave propagation model was presented that simulates the hemodynamical conditions directly after AVF creation [101]. In order to make personalized predictions that might assist the surgeon during vascular access planning, the model parameters were adapted to patient-specific conditions and the model was able to select the same AVF configuration (upper or lower arm) as an experienced surgeon (more than 1000 interventions) in nine out of ten patients. Furthermore, the predictions of the mean brachial flows were similar to ultrasound flow measurements in six out of ten patients [101]. Although these preliminary results were promising, to further optimize the use of this personalized pulse wave propagation model we need to be able to deal with the uncertainty in and incompleteness of input datasets obtained by clinical measurements. This uncertainty in input parameters will propagate to the pressure and flow predictions and therefore it is necessary to analyse the uncertainty in the predictions before inferences with the model can be made reliably. The knowledge of which model parameters influence output uncertainty the most can be used to determine which clinical measurements are most important for obtaining reliable predictions. Developing more accurate measurement methods for these model parameters will be most rewarding as this will result in the best improvement of the predictions. Model parameters that hardly influence the output can be fixed within their uncertainty interval (parameter fixing), whereas the model parameters that largely influence the output should be repetitively measured 
with high accuracy to reduce output uncertainty (parameter prioritization).

To select which model parameters can be fixed and which model parameters need to be prioritized for measurement improvements, a sensitivity analysis needs to be performed $[68,69]$. A sensitivity analysis can either be local or global [68, 69, 70, 102]. In a local sensitivity analysis, changes in the output are investigated by varying one input parameter around its initial value, while all other input parameters are kept fixed. The conclusions, therefore, only hold when the input conditions are kept around the initial state and neglect the influence of interactions between input parameters. In a global sensitivity analysis, sensitivity measures are determined while all model parameters are changed simultaneously and thus the complete input domain is sampled and interactions between model parameters are taken into account. Because the wave propagation model is nonlinear and interactions between parameters are expected, a global sensitivity analysis is the most suitable for our application.

Xiu et al. [71] performed uncertainty and sensitivity analysis in a wave propagation model of a human arterial tree based on generalized polynomial chaos expansion. The uncertain model parameters were modeled as random variables directly within the governing equations, transforming them into stochastic equations. This method gives a global description of the effect of model parameter uncertainties on the output. However, the deterministic properties of the model are lost and therefore apportioning the output uncertainty to the input uncertainties is difficult. An alternative is a Monte Carlo approach, in which the input parameters are randomly varied within their uncertainty domain [68, 69]. Leguy et al. [72] used Monte Carlo simulations to perform a global sensitivity analysis of a wave propagation model and showed that the majority of model parameters affected the output significantly. However, Leguy et al. used the Pearson correlation coefficient and the ranked Spearmann correlation coefficient as sensitivity measures [72]. These correlation coefficients can only be applied for monotonic models and do not reveal interactions between model parameters $[68,69,74,102]$. Variance-based methods are independent of the model's behavior with respect to linearity, monotonicity or additivity, and these methods also take into account possible interactions between parameters. Wenk et al. [39] used a variance-based method in their application of finite element stress analysis of atherosclerotic plaques. However, they only had a limited number of three input parameters. To our knowledge, variance-based methods have not yet been applied to wave propagation models which typically have 50 parameters. The aim of this study is to identify the influential and non-influential model parameters of our pulse wave propagation model, when applied to AVF surgery with respect to parameter fixing and parameter prioritization. We use a variance-based method and Monte Carlo simulations, which is considered to be 
the current best available practice to perform a global sensitivity analysis [68, 69, 75]. This analysis will generate more insight into the model and into which model parameters should be determined more accurately to reduce output uncertainty, resulting in an improved measurement protocol. Because the choice of which parameters to prioritize and which parameters to fix depends on the specific output parameter of interest, we have focused in this study on the mean brachial flow and systolic radial artery pressure distal to the anastomosis after AVF creation. These outputs are chosen as mean brachial flow is related to AVF short-term failure, while distal systolic pressure in the radial artery is used as measure for finger pressure, which is related to distal ischemia [7, 8, 9]. Radial pressure is chosen as it is difficult to assess vessel diameters and lengths in the hand vasculature.

In this paper, we briefly describe the distributed lumped parameter pulse wave propagation model, and, thereafter, present the variance-based global sensitivity analysis. In the description of the global sensitivity analysis, an overview of the model parameters and their uncertainty intervals are given. In addition, the computational framework to calculate the sensitivity indices is presented. Results with regard to mean brachial flow and finger pressure are presented and conclusions on how to improve the measurement protocol to obtain the most reliable model outcomes are drawn.

\subsection{Material and methods}

\subsubsection{The wave propagation model}

In this section, we give a short description of the distributed lumped parameter wave propagation model that we use. For details the reader is referred to Huberts et al. [101]. For the distributed lumped parameter wave propagation model in this study, the vascular tree is divided in edges that are connected based on anatomy (Figure 4.1). Each edge is divided into segments (Figure 4.2) with a maximum length of $5 \mathrm{~cm}$. In each segment the local relation between pressure and flow is described. For the scope of modeling the effect of vascular access creation on blood pressure and flow distribution, segments that represent arteries, veins and the anastomosis are needed (Figure 4.2). For arterial and venous segments, a lumped parameter approach based on local mass and momentum equations is applied [86, 101]. The anastomosis segment consists of nonlinear resistors that depend on anastomosis angle and blood flow. Parts of the cardiovascular system are truncated and terminated with three-element windkessel models [57]. Assembling the equations for all segments results in a system of differential equations that is solved by numerical inte- 
gration applying the trapezium rule for implicit time integration [101]. On the first aortic node an MR measured aortic flow is prescribed, whereas a fixed intravenous pressure is prescribed on the last venous node (Figure 4.1).

In this study two different topologies (computational domains) were used (Figure 4.1). The first topology consists of the main arteries of the right arm, i.e. the innominate artery, subclavian artery, axillary artery, brachial artery, interosseus artery, radial artery and ulnar artery. A truncated part of the aorta, of the vertrebral artery and of the right and left common carotid arteries are also included and the arterial tree is extended with an anastomosis segment and a venous outflow tract to mimic a lower arm AVF. The outflow tract consists of the cephalic vein in the upper and lower arm, the median cubital vein, the basilic vein in the upper arm and the axillary and subclavian vein. The second topology represents an arterial tree with an upper arm AVF. The outflow tract now consists of the basilic, axillary and subclavian vein.

\subsubsection{Variance-based method}

A variance-based method is used to determine the influence of the input parameters on the model output. The model output may be either a scalar or a vector but will be a scalar in this study as will be explained later in Section 4.2.3. Sensitivity indices, as described earlier by Saltelli et al. $[68,69,103]$ and outlined below, are used for parameter prioritization and parameter fixing. Parameter prioritization aims to identify the input parameter $X_{i}$ that results in the largest reduction in variance of the model output of interest $Y$ if the true value of this parameter would be known. For parameter prioritization we need to determine for each input parameter the variance $V(Y)$ in the model output of interest if this input parameter is fixed at its true value $x_{i}^{*}$, i.e. $V\left(Y \mid X_{i}=x_{i}^{*}\right)$ (see this chapter's Appendix for more details). Knowing the exact value of an influential parameter will result in $V\left(Y \mid X_{i}=x_{i}^{*}\right) \leq V(Y)$. However in general, the exact value of $X_{i}$ is not known and therefore $x_{i}^{*}$ can have different values in the domain of $X_{i}$. For this reason, the expected value of $V\left(Y \mid X_{i}=x_{i}^{*}\right)$ is determined by integrating this conditional variance, weighted by the probability density function of $X_{i}$, over all possible values $x_{i}^{*}$ (i.e. the domain $X_{i}$ ), which results in $E\left(V\left(Y \mid X_{i}\right)\right)$ (see $[68,69,103]$ and the Appendix of this chapter). It can be proven that [104],

$$
V(Y)=E\left(V\left(Y \mid X_{i}\right)\right)+V\left(E\left(Y \mid X_{i}\right)\right) .
$$

Thus the total unconditional variance, $V(Y)$, is the sum of the main effect, $V\left(E\left(Y \mid X_{i}\right)\right)$ and the residual effect $E\left(V\left(Y \mid X_{i}\right)\right)$ of $X_{i}$ on $Y$. By dividing the main effect by the total 


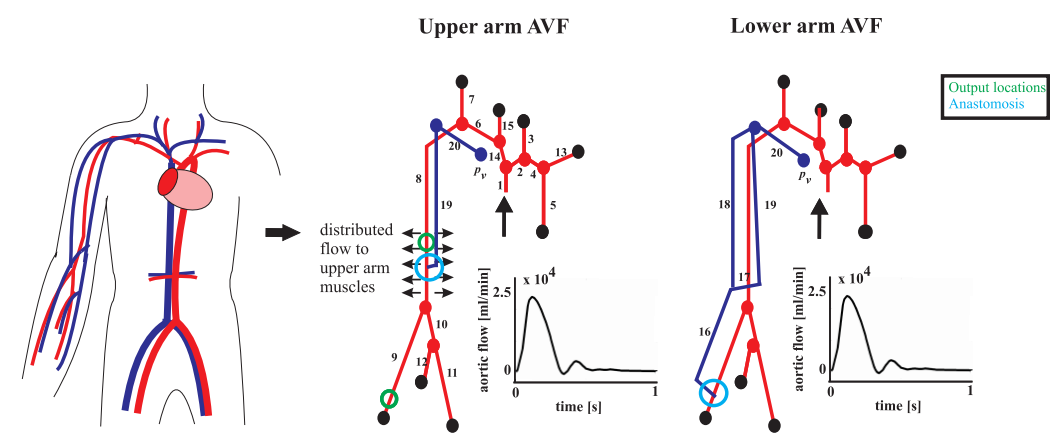

Figure 4.1: The computational domains for the two configurations. The numbers indicate to the edges presented in Table 4.2, whereas the green circles show the location where output waveforms are analyzed and the navy blue circles show the anastomosis. Note that the arterial edge numbers and the distributed flow are only shown in the upper arm AVF configuration. In the lower arm AVF configuration they are omitted to make the figure more clear.

Lumping the edges into segments

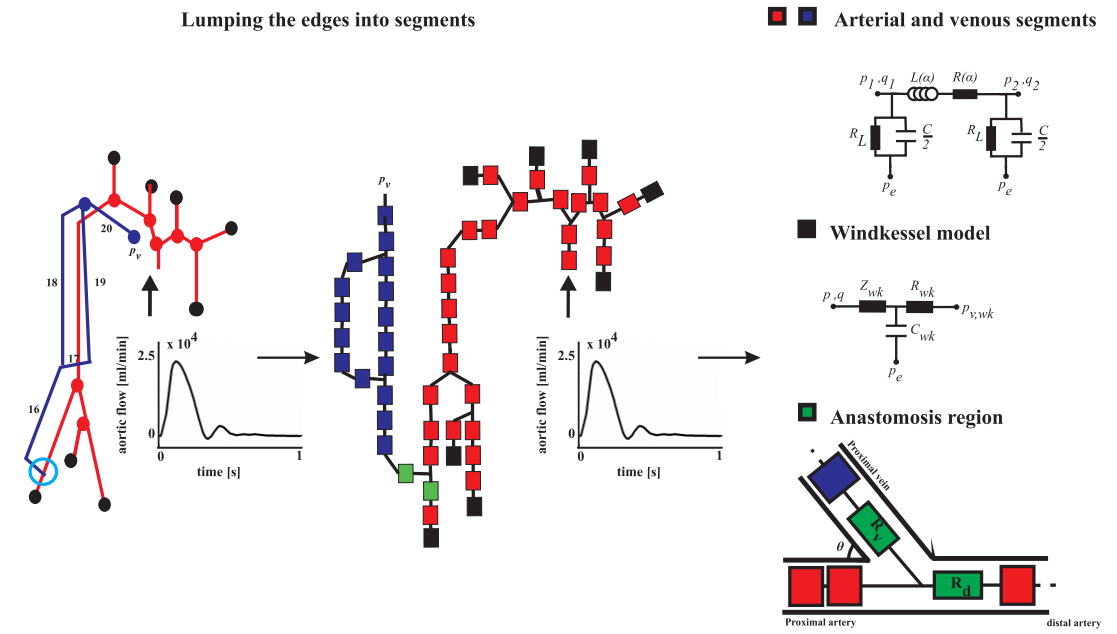

Figure 4.2: The vessel edges lumped into segments in case of the lower arm AVF (left). On the right side the lumped parameter models representing the arterial or venous segments (top), the windkessels (middle) and the anastomosis segments (down) are shown. Arterial and venous edges are build from the arterial and venous segments, whereas at the anastomosis region two anastomosis segments (flow dependent resistors) are inserted. Truncated arteries are closed by windkessels. 
unconditional variance, the main (effect) sensitivity index is given by

$$
S_{i}=\frac{V\left(E\left(Y \mid X_{i}\right)\right)}{V(Y)}=1-\frac{E\left(V\left(Y \mid X_{i}\right)\right)}{V(Y)}
$$

The main sensitivity index has a value between 0 and 1 and represents the expected reduction in output variance that one would obtain if the true value, albeit unknown, of the associated input parameter was known and was made into a model constant. A main sensitivity index which is large indicates an influential parameter. In case of additive models with $k$ input parameters, the total variance only consists of main effects of the input parameters $[68,69,105]$, i.e. $\sum_{i=1}^{k} S_{i}=1$.

For non-additive models, in which interactions between model parameters occur, determining the main sensitivity index is not sufficient to assess which parameter can be fixed within its uncertainty range because it is possible that the main effect of a parameter is small, while its contribution via the interaction with other terms is large. In that case $\sum_{i=1}^{k} S_{i} \leq 1$. For parameter fixing a sensitivity index which incorporates the effect of interactions is needed.

The missing fraction of the variance for a non-additive model can be retrieved by determining the main sensitivity index for a group of parameters, e.g., for parameter $X_{i}$ and $X_{j}$. When the combined main sensitivity index of these parameters is larger than the sum of the two individual main sensitivity indices, the extra effect $S_{i j}$ is the second-order or interaction effect of the two parameters, i.e.:

$$
S_{i j}=\frac{V\left(E\left(Y \mid X_{i}, X_{j}\right)\right)}{V(Y)}-\frac{V\left(E\left(Y \mid X_{i}\right)\right)}{V(Y)}-\frac{V\left(E\left(Y \mid X_{j}\right)\right)}{V(Y)} .
$$

Sobol [105] showed that for $k$ independent input parameters there may be interaction terms up to the $k^{t h}$ order and that all interaction terms add up to the $k^{\text {th }}$ order, i.e.:

$$
\sum_{i} S_{i}+\sum_{i} \sum_{i>j} S_{i j}+\sum_{i} \sum_{i>j} \sum_{l>j} S_{i j l}+\ldots+S_{12 \ldots k}=1 .
$$

Determining all $\left(\begin{array}{l}k \\ n\end{array}\right)$ sensitivity indices will result in the evaluation of many multi-dimensional integrals which will become computational expensive. To overcome this, total (effect) sensitivity indices are used $[68,69,106]$.

These total sensitivity indices can be determined from $V\left(E\left(Y \mid X_{1}, \ldots, X_{i-1}, X_{i+1}, \ldots, X_{k}\right)\right)=V\left(E\left(Y \mid X_{-i}\right)\right)$, which is the conditional variance if all parameters but $X_{i}$ are fixed. $V\left(E\left(Y \mid X_{-i}\right)\right)$ includes all terms in (4.4) that 
do not contain parameter $X_{i}$. Hence $1-\frac{V\left(E\left(Y \mid X_{-i}\right)\right)}{V(Y)}$ equals the sum of all terms (first and higher order) in (4.4) that do include $X_{i}$. By using (4.1), the total sensitivity index is derived:

$$
S_{T_{i}}=\frac{E\left(V\left(Y \mid X_{-i}\right)\right)}{V(Y)}
$$

In case of an non-additive model with three input parameters $\left(X_{1}, X_{2}\right.$ and $\left.X_{3}\right)$ the total sensitivity indices of these three input parameters are, $S_{T_{1}}=S_{1}+S_{12}+S_{13}+S_{123}$, $S_{T_{2}}=S_{2}+S_{12}+S_{23}+S_{123}$ and $S_{T_{3}}=S_{3}+S_{13}+S_{23}+S_{123}$ respectively. The total sensitivity index of parameter $X_{i}$ is thus the sum of the main sensitivity index and the higher-order sensitivity indices associated with parameter $X_{i}$. The total sensitivity index represents the expected variance that is left if all parameters but $X_{i}$ are exactly known and made into model constants. Varying model parameters with the largest total sensitivity indices influence the model output the most, whereas model parameters with the smallest total sensitivity indices can be fixed within their uncertainty range (parameter fixing).

The main and total sensitivity index can be estimated by performing a Monte Carlo experiment that explores the complete input space by randomly selecting a large number of input samples per input parameter $(>1000)[68,69]$. In this study, the main and total sensitivity indices are estimated for each input parameter by the method described by Saltelli et al. [103], which is summarized in this chapter's Appendix and makes use of a Monte Carlo experiment.

\subsubsection{Global sensitivity analysis}

The variance-based sensitivity analysis by means of a Monte Carlo experiment is performed on six patient-specific datasets to examine the impact of different initial values on the results. The datasets contain the diameters, wall thicknesses, Young's moduli and the eccentricity ratios (quotient of the major and minor axis diameter) of all edges given in Table 4.2 as well as edge lengths. In addition, mean brachial pressure, distributed flow to the muscles in the upper arm, mean aortic flow, the anastomosis angle, intravenous pressure and windkessel parameters are included in the datasets as model parameters. Details on the datasets and the measurements can be found in Huberts et al. [101]. Varying the input parameters of all vascular segments independently within their uncertainty domains would result in non-physiological outputs in the Monte Carlo experiment, due to non-physiological transitions between connecting vessels (e.g. a small diameter vessel continuing in a larger diameter vessel). Therefore, we decided to vary the input parameters of vessel tracts simultaneously. This results in the set of input parameters given in 
Table 4.1. The uncertainty domains of all parameters are also given in Table 4.1. 
Table 4.1: The input parameters and their uncertainty intervals. Edges that are changed simultaneously are separated by commas. Note that for the upper arm AVF some venous edges are not in the model and therefore the related parameters are by definition non-influential in the upper arm AVF configuration.
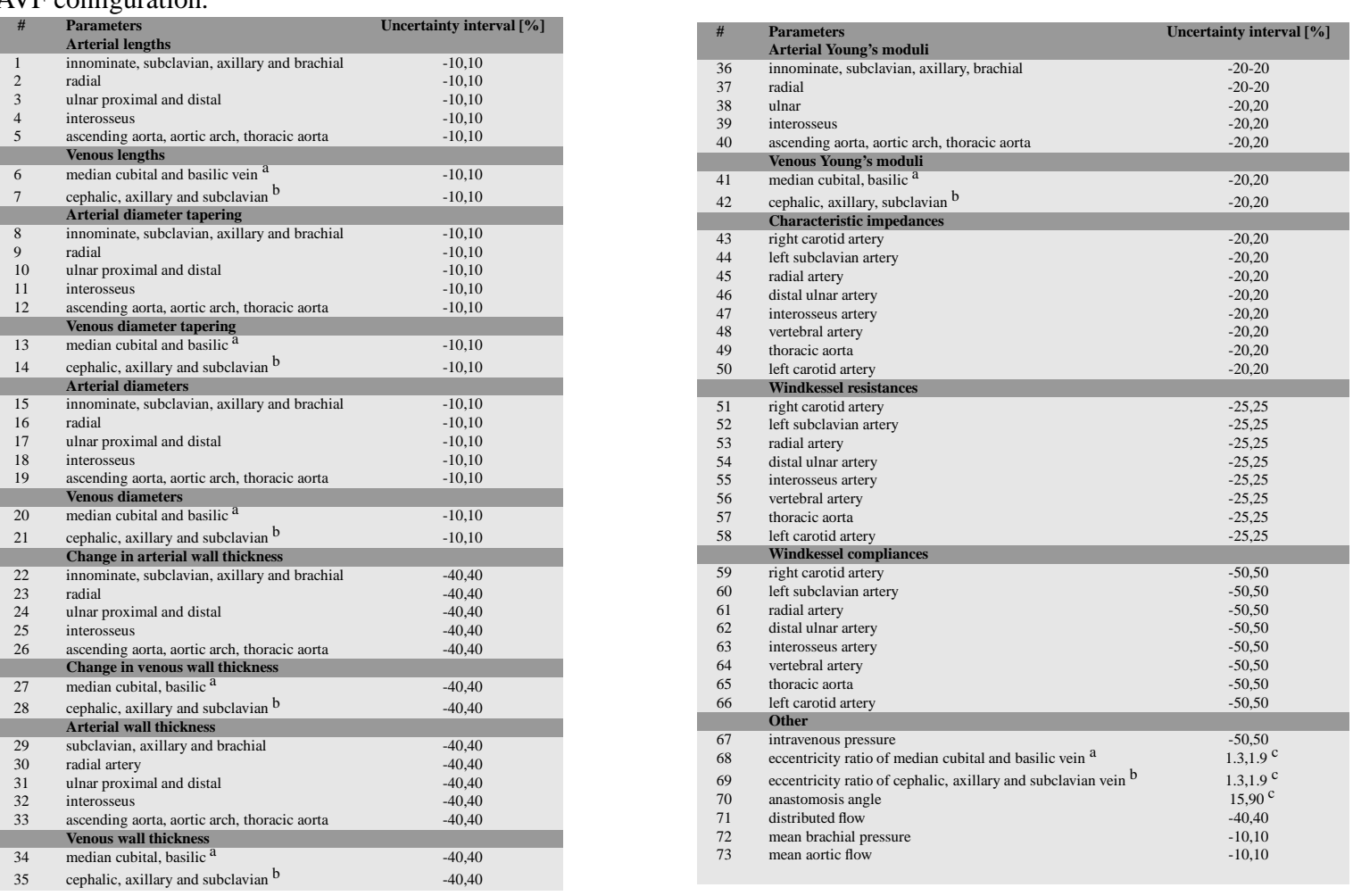

$\mathrm{a}_{\text {For an upper arm AVF the basilic, axillary and subclavian vein are combined. }}$

$\mathrm{b}_{\text {Not used in the model for an upper arm AVF }}$

c The absolute value [-] is presented. 
Table 4.2: The edge numbers and their corresponding vessels.

\begin{tabular}{cl}
\hline$\#$ & Vessel name [-] \\
\hline 1 & Ascending aorta \\
2 & Aortic arch A1 \\
3 & Left carotid artery \\
4 & Aortic arch A2 \\
5 & Aortic arch A3 and Thoracic aorta \\
6 & Subclavian artery and Axillary artery A \\
7 & Vertebral artery \\
8 & Axillary artery B and Brachial artery \\
9 & Radial artery \\
10 & Proximal ulnar artery \\
\hline
\end{tabular}

\begin{tabular}{|cl|}
\hline$\#$ & Vessel name [-] \\
11 & Distal ulnar artery \\
12 & Interosseus artery \\
13 & Left subclavian artery \\
14 & Innominate artery \\
15 & Right carotid artery \\
16 & Distal cephalic vein \\
17 & Median cubital vein \\
18 & Proximal cephalic vein \\
19 & Basilic vein and axillary vein B \\
20 & Axillary vein A and Subclavian vein \\
\hline
\end{tabular}

\section{Input parameters: definition of uncertainty domains}

In clinical practice, it is not common to perform repeated measurements and usually only one measurement is performed for each variable. Therefore, the exact uncertainty distributions are unknown. For this reason, we assumed an uniform uncertainty distribution for each input parameter. This is a worst case scenario as it will result in an overestimation of the effect of each parameter uncertainty. The uncertainty domain used for each model parameter is based on measurement uncertainties or variations reported in literature and is given below per input parameter. Uncertainties resulting from biological variations over time are neglected in the current study. Furthermore, we assume that all input parameters are measurable and that the initial values are representative for the actual state of the patient.

\section{Geometry}

Arterial and venous lengths. MRI or other imaging modalities are available in clinics to extract information from the vascular tree. However, within the clinical routine of vascular access planning, ultrasound examination is the preferred preoperative modality for vessel assessment. With ultrasound it is difficult to measure vessel lengths and therefore we decided to base the vessel lengths in this study on a generic geometrical dataset for a subject with a body height equal to $175 \mathrm{~cm}$ which was previously used by Westerhof et al. [36]. Because this generic dataset only includes arterial vessel lengths, we assumed that the venous lengths are equal to the arterial lengths at each anatomical location. By assuming that vessel lengths are proportional to the height of a person, the maximum and minimum value of the uncertainty domain were derived.

The Dutch Association of Statistics (CBS; www.cbs.nl) reported in 2010 that the majority $80 \%$ of the Dutch population older than 45 years has a body height between $163 \mathrm{~cm}$ and $188 \mathrm{~cm}$. Only $5 \%$ of the population is taller than $188 \mathrm{~cm}$ while $15 \%$ is smaller than 163 
$\mathrm{cm}$. We therefore chose, by taking also people with lengths between 158 and $193 \mathrm{~cm}$ into account, an uncertainty of $10 \%$ in body height compared to the $175 \mathrm{~cm}$ of the generic dataset. By assuming proportionality between vessel length and body height, we used an inaccuracy of $10 \%$ for the arterial and venous vessel lengths.

Arterial and venous diameters. For arterial diameters Leguy et al. [72] determined an absolute inaccuracy for ultrasound measurements of approximately $150 \mu \mathrm{m}$ based on intra-subject variability in M-line brachial diameter measurements. This accuracy is of the order of one ultrasound wavelength for a $10 \mathrm{MHz}$ ultrasound probe, which is the best obtainable accuracy with such a probe [107]. The smallest vessel in our application is $1.5 \mathrm{~mm}$ in diameter, which results in a measurement uncertainty of $10 \%$ for the diameter. Hence as maximum variation, $10 \%$ is applied in our study both for arterial and venous diameters.

Arterial and venous diameter tapering. Within an edge a linear diameter tapering in both arteries and veins is assumed. The influence of tapering is investigated by changing the smallest diameter of an edge while changing the largest diameter in the opposite direction. The uncertainty interval for these changes are based on $10 \%$ of the smallest diameter.

Arterial and venous wall thickness. Molinari et al. [108] reported intima-media thickness (IMT) measurement errors in the carotid artery ranging from 10 to $150 \mu \mathrm{m}$, depending on the segmentation method and the ultrasound scanner. In the brachial artery the measurement error is expected to be larger because the image quality is lower compared to carotid artery images, which is a result from the anatomical location (depth) and size. The brachial IMT is about $400 \mu \mathrm{m}[24,95]$. A measurement error of $150 \mu \mathrm{m}$ thus results in $38 \%$ uncertainty. Therefore, for the wall thickness the maximum variation was set to $40 \%$.

Change in wall thickness. Within an edge the wall thickness is assumed to change linearly. The same approach is used as for diameter tapering, resulting in a variation of $40 \%$ for wall thickness change within an edge.

Eccentricity ratio. Planken et al. [94] performed venous diameter measurements on ten healthy male volunteers at different congestion pressures. This resulted in an average venous eccentricity ratio between 1.3 and 1.9. This range is applied in our sensitivity study. For the arteries the eccentricity ratio is assumed to be fixed at 1 (i.e. circular) 
Anastomosis angle. The anastomosis angle for upper arm AVFs is close to 90 degrees, whereas for lower arm AVFs anastomosis angles smaller than 90 degrees are observed [30]. In the sensitivity analysis this parameter is varied between 15 and 90 degrees.

\section{Mechanical vessel properties and distributed flow to small side-branches}

Arterial and venous Young's modulus. Leguy et al. [72] performed measurements of the Young's modulus on brachial arteries of young healthy volunteers and reported an uncertainty range of $20 \%$. In our sensitivity study this variation is applied for the arterial and venous Young's moduli.

Distributed flow in the brachial artery. In the wave propagation model distributed flow through small side-branches of the brachial artery is incorporated. This distributed flow is determined from the difference between axillary artery flow (MRI) and the sum of the radial artery flow (MRI), ulnar artery flow (MRI) and interosseus artery flow (mass conservation). Lotz et al. [109] reported a measurement error for flow in the aorta with MRI of approximately $10 \%$. We assume that in the peripheral arteries this uncertainty is higher because vessels are smaller and thus less pixels per diameter are available. Therefore, an error of approximately $15 \%$ is assumed for the axillary, ulnar, radial and interosseus artery flow measurements. The uncertainty in the distributed flow is calculated by summation of the four absolute errors. For the datasets of six ESRD patients [101] this results in an average absolute error of approximately $35 \mathrm{ml} / \mathrm{min}$, whereas the distributed flow is $88 \mathrm{ml} / \mathrm{min}$. Hence in the sensitivity analysis, a relative error of $40 \%$ is taken.

Mean brachial pressure. The mean brachial pressure is assessed by using NexFin (BMEYE B.V., The Netherlands). For the NexFin, systolic and brachial blood pressure were validated against the Riva-Rocci method and differences were respectively $4 \%$ and $3 \%$ [110]. A variance of $10 \%$ is applied for the blood pressure to be on the safe side.

\section{Boundary conditions}

Aortic flow. The measurement error on MR flow measurements in the aorta is approximately $10 \%$ [109]. We therefore varied the mean aortic flow with a maximum of $10 \%$ by scaling the systolic part of the aortic flow waveform.

Characteristic impedance. In accordance with Leguy et al. [72], for the characteristic impedance an uncertainty of $20 \%$ is used. 
Peripheral resistance. The peripheral resistance is determined from the quotient between mean arterial pressure and mean flow. The uncertainty in mean arterial pressure is $10 \%$, whereas the uncertainty in the radial and ulnar flow is $15 \%$. Therefore, an uncertainty of $25 \%$ in the peripheral resistance is applied.

Peripheral compliance. Peripheral compliance is defined as the quotient of the time constant (describing the descending slope in diastole) and the peripheral resistance. According to Leguy et al. [72], the uncertainty in the time constant is $27 \%$, whereas the uncertainty of the peripheral resistance is $25 \%$. Therefore, the uncertainty interval of the peripheral compliance is set to $50 \%$

Intravenous pressure. Guyton et al. [1] reported that the intravenous pressure is about $20 \mathrm{mmHg}$ directly behind the capillary beds and decreases to $0 \mathrm{mmHg}$ in the venae cava. In addition, Strandness and Sumner [99] state that $15 \mathrm{~cm}$ after the anastomosis the venous pressure returns to normal venous pressure and that venous pressure ranges from 0 to 15 $\mathrm{mmHg}$. Furthermore, they state that pulse pressure after AVF creation seldom exceeds 5 $\mathrm{mmHg}$. The venous pressure is thus varied between $5-15 \mathrm{mmHg}$.

\section{Monte Carlo experiment}

To calculate all sensitivity indices $N(k+2)$ Monte Carlo runs are needed [103], in which $N$ is the number of runs per model parameter. A convergence check of the sensitivity estimates (Appendix of this chapter) determined $N$ to be 5000. In this case, the sensitivity indices have an uncertainty of maximum 0.05 . Because the distributed lumped parameter pulse wave propagation model in this study has 73 input parameters, 375000 Monte Carlo runs are needed to estimate the sensitivity indices for each patient-specific model. For each run the uncertainty domains of all input parameters are sampled, resulting in a unique set of input parameters for each run.

\section{Parameter sampling}

All input parameters in this study are assumed to be independent of each other, which makes it possible to generate the needed samples for each parameter by exploring the marginal distribution of each input parameter by means of stratified Latin Hypercube sampling [111]. This method is chosen because it generates samples that are homogeneously distributed over the complete input space [111]. The samples are generated with 
Matlab (Mathworks Inc) within the domain $[0,1]$ and for each parameter mapped on its uncertainty interval.

\section{Computing facilities}

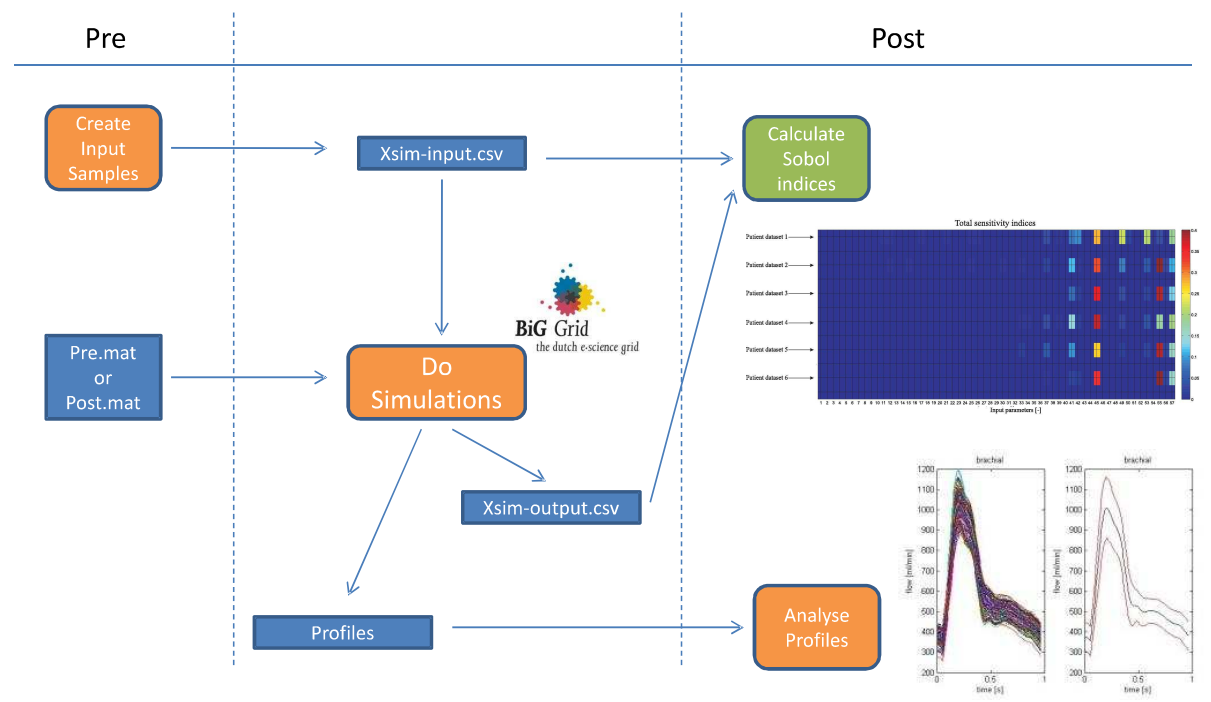

\begin{tabular}{|l|l|}
\hline Xsim-input.csv & each row defines the variation to be applied on the input-parameters \\
\hline Xsim-output.csv & $\begin{array}{l}\text { each row contains the values of various output-parameters calculated with input-parameter values } \\
\text { defined in the same row in Xsim-input.csv }\end{array}$ \\
\hline Profiles & Contains the pressure- and flow-profiles of the nodes from which the output-parameters are taken \\
\hline & Done in "R" \\
\hline & Done in "matlab"
\end{tabular}

Figure 4.3: The computational framework.

The sensitivity analysis for one AVF configuration for all six patient-specific datasets results in more than two million simulations. One simulation including storage of the waveforms, takes on average 20 seconds, which for two million simulations would result in more than 500 days of calculation on a single central processing unit (CPU). This long calculation time together with the time needed for the processing and collecting of data would hamper the practical use of Monte Carlo simulations. Therefore, we resorted to Grid computing. A framework is developed by using the BigGrid central facility (www.biggrid.nl) at Philips Research B.V. (Eindhoven, The Netherlands). This gave us 
access to multiple processors (1000 CPU) simultaneously, which reduces the total time of the sensitivity analysis to half a day. The framework is shown in Figure 4.3. The sensitivity indices are computed in several steps. In the first step input datasets are generated in Matlab (Mathworks Inc) by means of Latin Hypercube sampling (discussed earlier) and these samples are transformed into the input format required by the model. Next, the input is fed to the wave propagation model (Matlab on BigGrid) and the model calculates and stores pressure and flow waveforms at seven locations: the aorta, the axillary, brachial, proximal radial, distal radial, proximal ulnar and distal ulnar artery. When all simulations are ready and all pressure and waveforms are stored, data are consolidated into twelve scalar properties for each arterial location: the maximum, the minimum, the pulse (maximum-minimum), the maximal derivative and the time-averaged value of both pressure and flow waveforms. In addition, the transit time defined as the foot-to-foot traveling time from heart contraction to the specified waveform is also computed. The timing of the specified waveform is determined at $10 \%$ of the maximum pulse. These scalars are finally fed into Statistical software (R-software) that calculates the sensitivity indices.

\section{Simulation and analysis}

The computational framework is firstly validated by applying it to the Sobol G-function, which is a strongly non-monotonic, non-additive function of $k$ parameters $x_{i}$, that are assumed to be identically and uniformly distributed in the unit hypercube $\left\{I^{k}=x \mid 0 \leq x_{i} \leq 1 ; i=1,2, \ldots, k\right\}$ and which is often used in literature to benchmark the calculation of sensitivity indices $[75,103,112,113]$. For details about the benchmarking we refer to this chapter's Appendix.

Thereafter, the Monte Carlo experiment on the distributed lumped parameter wave propagation model is executed. To be able to identify the parameters that could best be measured more accurately to reduce output variance the most, the main sensitivity indices are used, whereas total sensitivity indices are used to determine which parameters could be fixed (Figure 4.4). To study if parameters are involved in interactions, the difference between the total and main sensitivity indices is also considered. A threshold of 0.05 is taken to define a parameter as influential. As output of interest, we focused on the mean brachial flow and systolic pressure distal to the anastomosis as these are the most important output parameters for our application. Results obtained for other output parameters are therefore not included in this manuscript. Before the sensitivity indices are calculated, we manually checked whether the output scalars are within physiological ranges. 


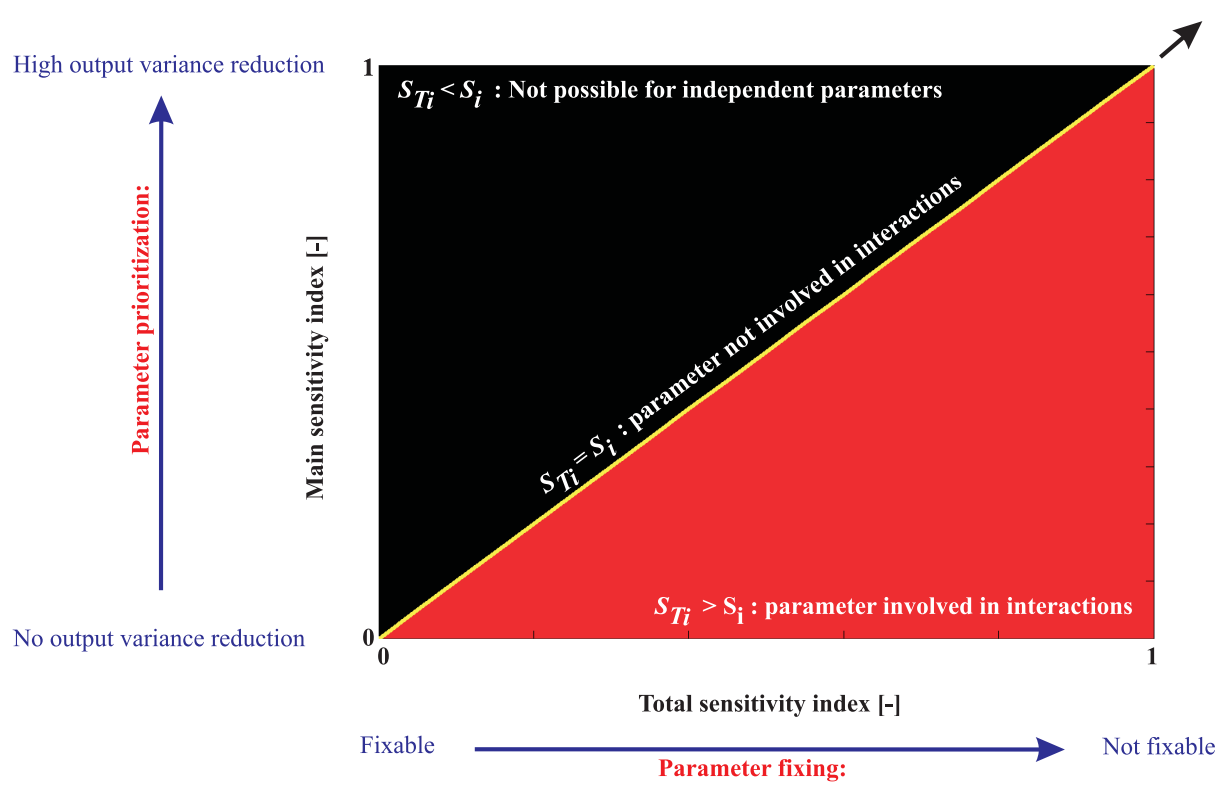

Figure 4.4: A schematic overview to clarify the interpretation of the main and total sensitivity indices and the sensitivity analysis setting they are used for.

\subsection{Results}

In this section we will present the results of the sensitivity analysis. The important model parameters with respect to parameter prioritization and parameter fixing will be identified. Color maps are used to show the most influential parameters for each patient-specific dataset because in these color maps differences between patients can easily be observed. Additionally, tables are used for further quantification of the sensitivity indices. In this section the results are described in detail for both a lower arm and upper arm AVF, while the most important results are highlighted and summarized in Table 4.3-4.5. 
Table 4.3: The expected reduction in output variance (in \%, thus hundred times the main sensitivity index) for all model parameters for which the main sensitivity index with respect to the mean brachial flow or the distal systolic pressure is larger than 0.05 for at least one of the six patients (P1 to P6). If the parameter is significantly influencing both brachial flow and distal systolic pressure in both configurations, the parameter is highlighted in bold.

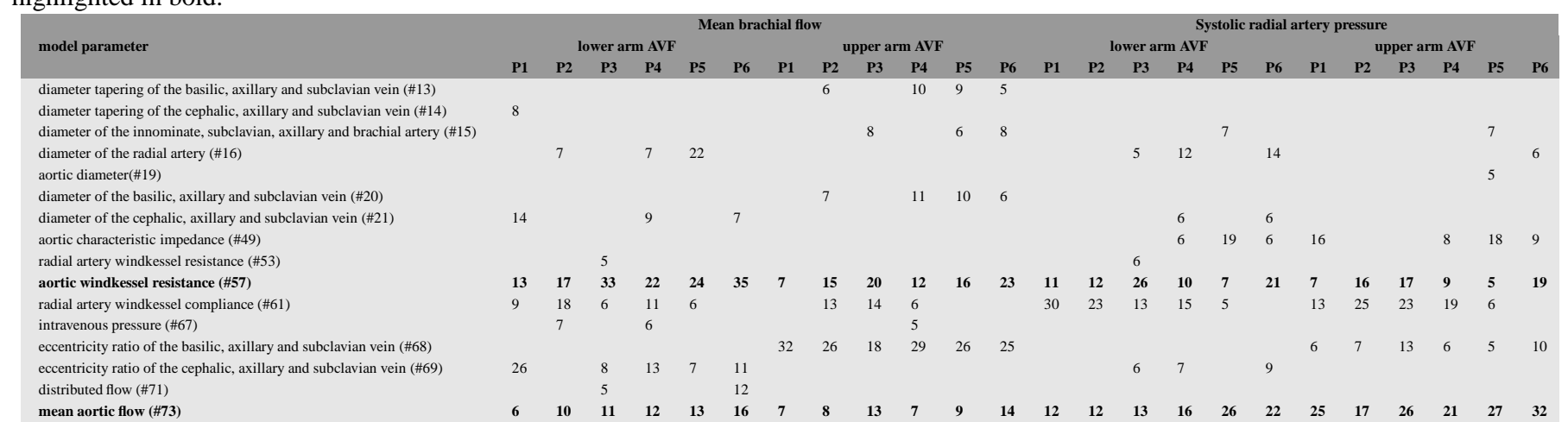


Table 4.4: The total sensitivity indices for model parameters that cannot be fixed within their uncertainty interval. They are larger than 0.05 for at least one of the six patients (P1 to P6). The residual variances are given in percentages (\%). If the parameter cannot be fixed for both brachial flow and distal systolic pressure in both AVF configurations, the parameter is highlighted in bold.

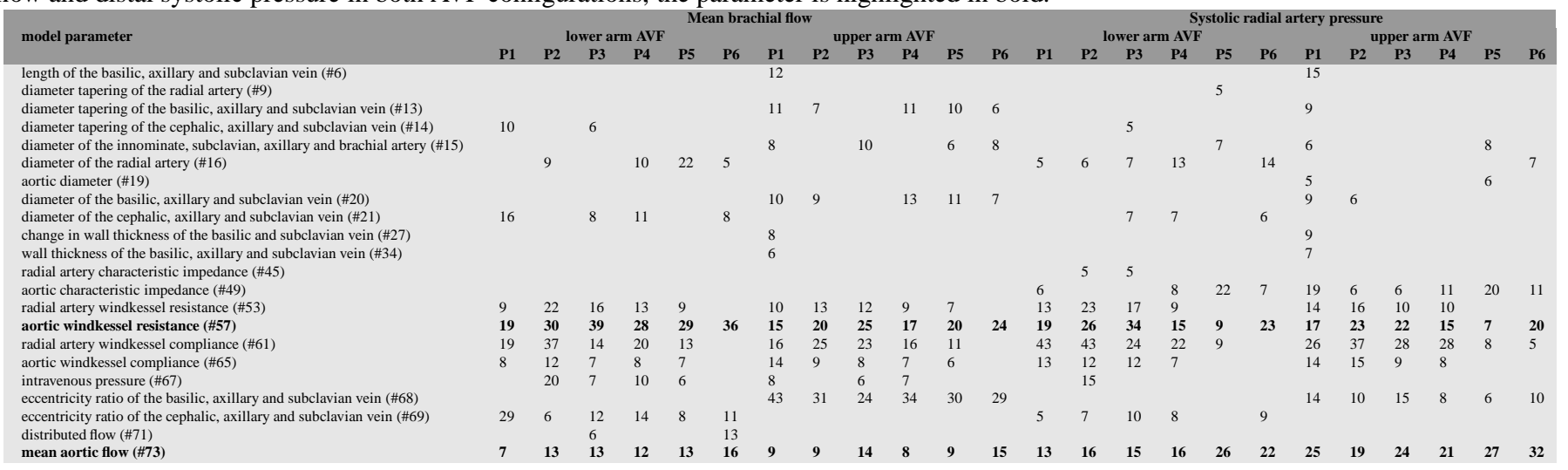


Table 4.5: The model parameters that interact with other parameters because the difference between main and total sensitivity index with respect to the mean brachial flow or the distal systolic pressure is larger than 0.05 for at least one of the six patients (P1 to P6). Differences are given in percentages $(\%)$.

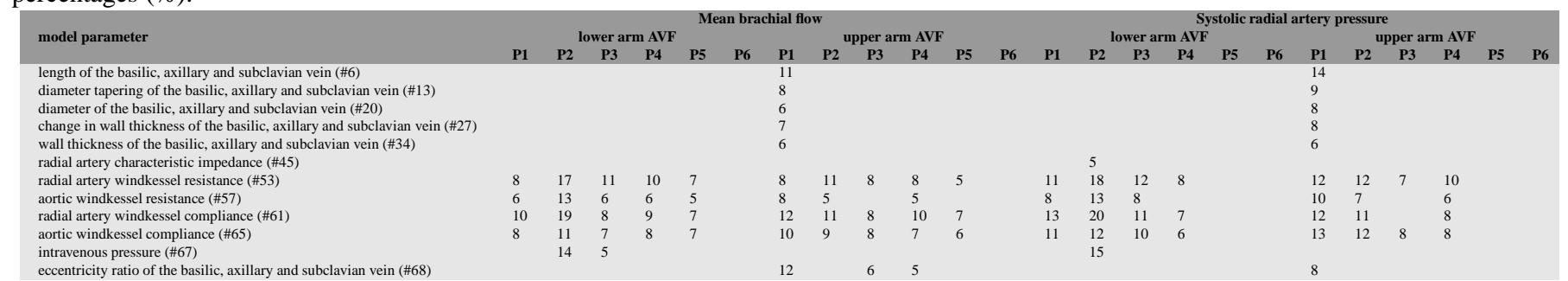




\subsubsection{Mean brachial flow}

\section{Lower arm AVF}

The uncertainty (coefficient of variation) in the predicted mean brachial flow for the six patients ranged from $15 \%$ to $25 \%$ in case of a lower arm AVF.

Figure 4.5 (top) shows that in all six patients the aortic windkessel resistance (\#57) and the mean aortic flow (\#73) have significant $(>0.05)$ main sensitivity indices for the the mean brachial flow in case of a lower arm AVF. Knowing these parameters could reduce output variance by $13-35 \%$ and $6-16 \%$, respectively (Table 4.3 ). Significant main sensitivity indices are furthermore observed (Figure 4.5, top) in most patients for the radial artery windkessel compliance (\#61, output variance reduction $0-18 \%$ ) and the eccentricity ratio of the cephalic, axillary and subclavian vein (\#69, 0-26\%). In addition, six model parameters have a significant main sensitivity index for only a few patients (Figure 4.5 (top) and Table 4.3). These are diameter tapering in the cephalic, axillary and subclavian vein (\#14), the radial artery radius (\#16), the cephalic, axillary and subclavian venous radius (\#21), the radial artery windkessel resistance (\#53), the intravenous pressure (\#67), and the distributed flow (\#71).

The model parameters that have a significant main sensitivity indices for brachial flow also have significant total sensitivity indices (Figure 4.5 (middle)). In all six patients the aortic windkessel resistance (\#57, 19-39\%), the eccentricity ratio of the cephalic, axillary and subclavian vein (\#69, 6-29\%) and the mean aortic flow (\#73, 7-16\%) have significant total sensitivity indices (Table 4.4). The radial artery windkessel resistance (\#53, $0-22 \%)$, the radial artery windkessel compliance (\#61, 0-37\%) and the aortic windkessel compliance (\#65, 0-12\%) have significant total sensitivity indices in most patients (Table 4.4) while the diameter tapering in the cephalic, axillary and subclavian vein (\#14), the radial artery radius (\#16), the cephalic, axillary and subclavian venous radius (\#21), the intravenous pressure (\#67), and the distributed flow (\#71) have significant total sensitivity indices for at most four patients.

When the total sensitivity index is larger than the main sensitivity index this indicates that the model parameter is significantly interacting with other model parameters. This holds for the radial artery windkessel resistance (\#53), the aortic windkessel resistance (\#57), the radial artery windkessel compliance (\#61) and the aortic windkessel compliance (\#65) in five out of six patients and for the intravenous pressure (\#67) in two out of six patients. 

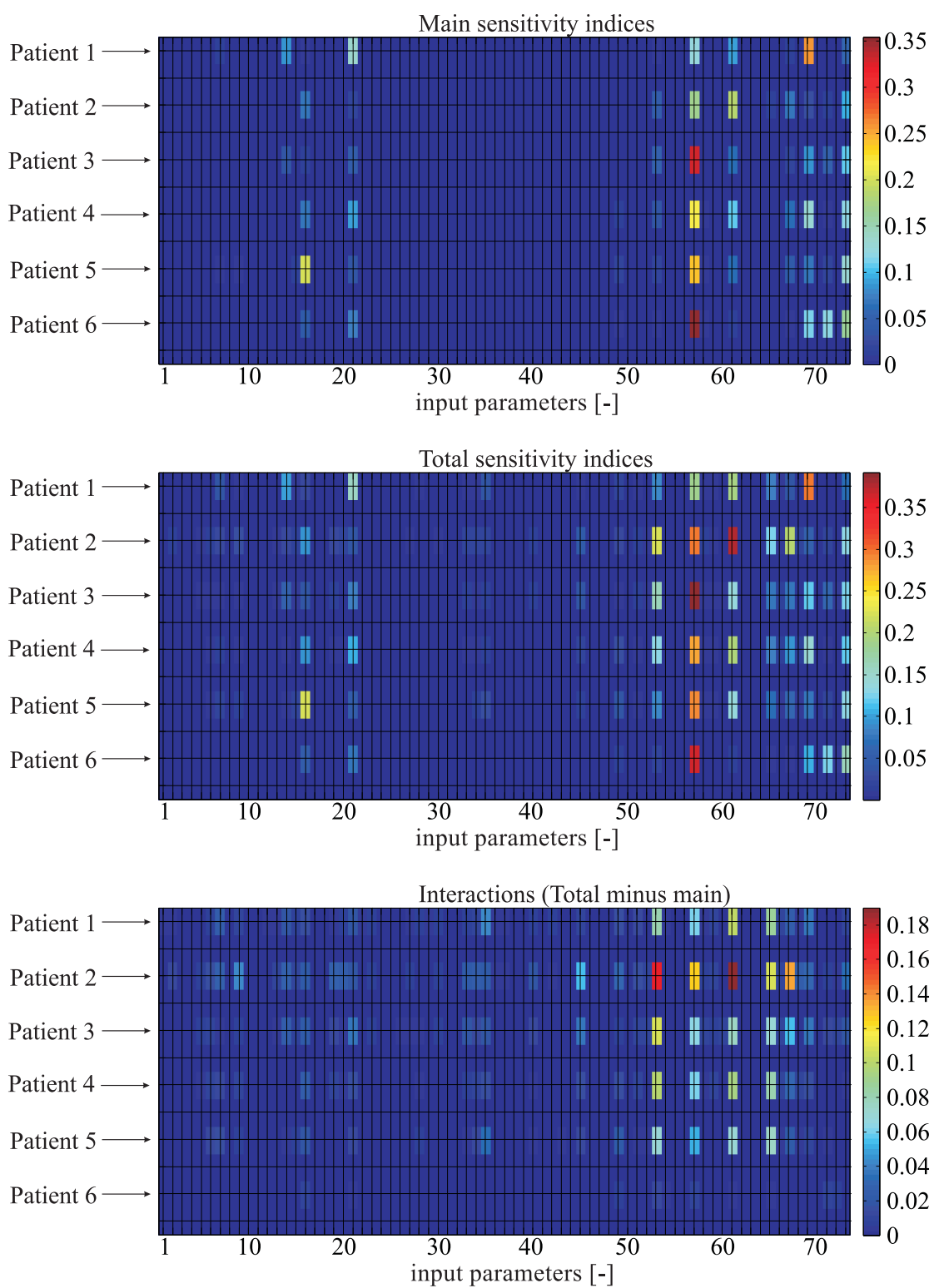

Figure 4.5: The total and main sensitivity indices and their difference for all input parameters with respect to the mean brachial flow in the lower arm AVF configuration. 


\section{Upper arm AVF}

The uncertainty in the predicted mean brachial flow for the six patients in case of an upper arm AVF is $13 \%$ to $21 \%$.

In all six patients the aortic windkessel resistance (\#57, expected output reduction of 7$23 \%)$, the mean aortic flow (\#73, 7-14\%) and the eccentricity ratio of the basilic, axillary and subclavian vein (\#68, 18-32\%)) have significant main sensitivity indices (Figure 4.6 (top) and Table 4.3). Five other model parameters have significant main sensitivity indices for only a few patients. These are the diameter tapering of the basilic, axillary and subclavian vein (\#13), the radius of the innominate, subclavian, axillary and brachial artery (\#15), the basilic, axillary and subclavian vein radius (\#20), the radial artery windkessel compliance (\#61) and the intravenous pressure (\#67).

Figure 4.6 (middle) and Table 4.4 show that for all six patients the aortic windkessel resistance ( $\# 57$, residual variance of $15-25 \%$ ), the eccentricity ratio of the basilic, axillary and subclavian vein (\#68, 24-43\%) and the mean aortic flow (\#73, 8-15\%) have significant total sensitivity indices. The diameter tapering of the basilic, axillary and subclavian vein (\#13, 0-11\%), the basilic, axillary and subclavian vein radius (\#20,0-13\%) and the radial artery windkessel compliance (\#61, 0-25\%), the radial artery windkessel resistance (\#53, 0-13\%) and the aortic windkessel compliance (\#65, 0-14\%) have significant total sensitivity indices in most patients. The radius of the innominate, subclavian, axillary and brachial artery (\#15), the intravenous pressure (\#67), the length (\#6), the wall thickness tapering (\#27) and the wall thickness (\#34) of the basilic, axillary and subclavian vein have significant total sensitivity indices for at most four patients.

From Figure 4.6 (bottom) and Table 4.5 it can be seen that significant interactions occur for the radial artery windkessel resistance (\#53), the radial artery windkessel compliance (\#61) and the aortic windkessel compliance (\#65) in five out of six patients. The aortic windkessel resistance (\#57), the eccentricity ratio (\#68), the length (\#6), the diameter tapering (\#13), the radius (\#20), the wall thickness tapering (\#27) and the wall thickness (\#34) of the basilic, axillary and subclavian vein are involved in at most halve of all six patients. 

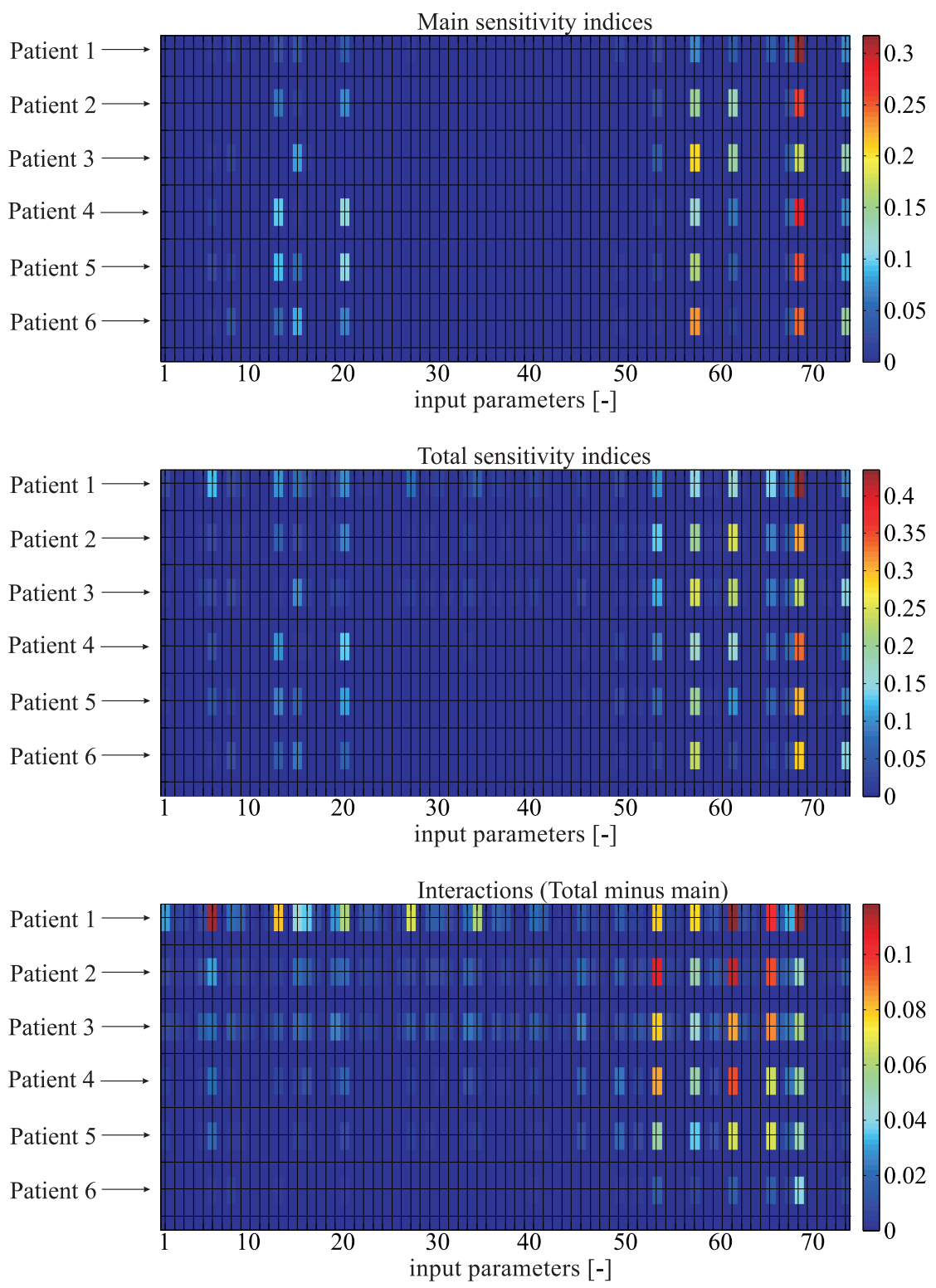

Figure 4.6: The total and main sensitivity indices and their difference for all input parameters with respect to the mean brachial flow in the upper arm AVF configuration. 


\subsubsection{Systolic pressure of the distal radial artery}

\section{Lower arm AVF}

The uncertainty in the predicted systolic radial artery pressure for the six patients in case of a lower arm AVF is $12 \%$ to $21 \%$.

In Figure 4.7 (top) and Table 4.3 it can be seen that for all six patients the aortic windkessel resistance (\#57) and the mean aortic flow (\#73) have significant main sensitivity. Knowing this parameters could reduce the output variance by $7-26 \%$ and by $12-26 \%$, respectively. The radial artery windkessel compliance (\#61, expected reduction in output variance of $0-30 \%$ ) has significant main sensitivity indices in five out of six patients, whereas in maximum halve of all six patients the radius of the innominate, subclavian, axillary and brachial artery (\#15), the radial artery radius (\#16), the cephalic, axillary and subclavian vein (\#21), the aortic characteristic impedance (\#49), the radial artery windkessel resistance (\#53) and the eccentricity ratio of the cephalic, axillary and subclavian vein (\#69) have significant main indices.

In Figure 4.7 (middle) and Table 4.4 fourteen model parameters have significant total sensitivity indices for at least one out of six patients. The aortic windkessel resistance (\#57, residual variance of 9-34\%) and the mean aortic flow (\#73, 13-26\%) have significant total sensitivity indices for all six patients. Significant total sensitivity indices are observed for the radial artery radius (\#16, 0-14\%), the radial artery windkessel compliance (\#61, $0-43 \%)$ and the eccentricity ratio of the cephalic, axillary and subclavian vein (\#69, 0 $10 \%)$ in five out of patients, whereas the aortic characteristic impedance (\#49, 0-22\%), the radial artery windkessel resistance (\#53, 0-23\%) and the aortic windkessel compliance (\#65, 0-13\%) have significant total sensitivity indices for four out of six patients. The radius of the innominate, subclavian, axillary and brachial artery (\#15), radius of the cephalic, axillary and subclavian vein (\#21), the diameter tapering of the radial artery (\#9), the diameter tapering of the cephalic, axillary and subclavian vein (\#14), the radial artery characteristic impedance (\#45) and the intravenous pressure (\#67) have significant total sensitivity index for at most three patients.

The radial artery windkessel resistance (\#53), the radial artery windkessel compliance (\#61) and the aortic windkessel compliance (\#65) are involved in significant interactions in four out of six patients (Figure 4.7 (bottom) and Table 4.5), whereas the aortic windkessel resistance (\#57) is involved in significant interactions in three out of six patients. The radial artery characteristic impedance (\#45) and the intravenous pressure (\#67) are only involved in interactions in one out of six patients. 

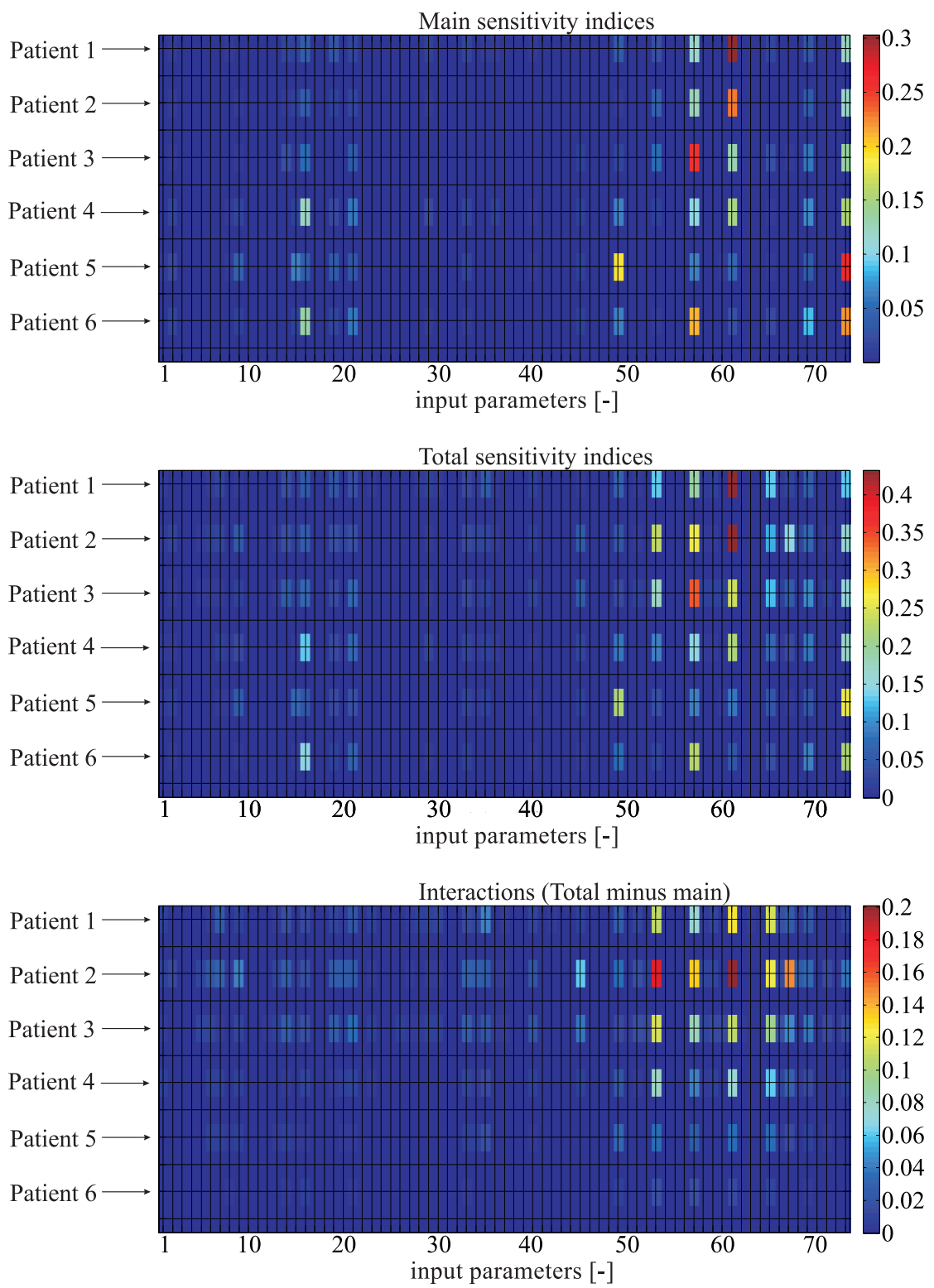

Figure 4.7: The total and main sensitivity indices and their difference for all input parameters with respect to the distal systolic pressure in the lower arm AVF configuration. 


\section{Upper arm AVF}

The uncertainty in the predicted systolic radial artery pressure for the six patients in case of an upper arm AVF is $11 \%$ to $14 \%$.

Figure 4.8 (top) and Table 4.3 show that for all six patients the aortic windkessel resistance (\#57, expected output variance reduction of 5-19\%), the eccentricity ratio of the basilic, axillary and subclavian vein $(\# 68,5-13 \%)$ and the mean aortic flow (\#73, 17 $32 \%$ ) have significant main sensitivity indices. The radial artery windkessel compliance (\#61, 0-25\%) has significant main sensitivity indices in five out of six patients, whereas aortic characteristic impedance (\#49, 0-18\%) has significant main sensitivity indices in four out of six patients. The radius of the innominate, subclavian, axillary and brachial artery (\#15), the radial artery radius (\#16) and the aortic radius (\#19) have significant main sensitivity indices for only one out of six patients.

In Figure 4.8 (middle) and Table 4.4 it can be observed that five parameters have significant total sensitivity indices for all six patients. These are the aortic characteristic impedance (\#49, residual variance of 6-20\%), the aortic windkessel resistance (\#57, 7$23 \%$ ), the radial artery windkessel compliance (\#61, 5-37\%), the eccentricity ratio of the basilic, axillary and subclavian vein $(\# 68,6-15 \%)$ and the mean aortic flow (\#73, 19$32 \%$ ). Next to these model parameters, the radial artery windkessel resistance (\#53) and the aortic windkessel compliance (\#65) have significant total sensitivity indices in four out of six patients, whereas the radius of the innominate, subclavian, axillary and brachial artery (\#15), the radial artery radius (\#16), the aortic radius (\#19), the length (\#6), the diameter tapering (\#13), the radius (\#20), the wall thickness tapering (\#27) and the wall thickness (\#34) of the basilic, axillary and basilic vein have significant total sensitivity indices in maximum two out of six patients.

The radial artery windkessel resistance (\#53) and the aortic windkessel compliance (\#65) are involved in significant interactions with other model parameters in four out of six patients, as can be seen in Figure 4.8 (bottom) and Table 4.5. In the same figure it can be seen that the aortic windkessel resistance (\#57) and the radial artery windkessel compliance (\#61) are involved in interactions in three out of six patients, whereas this holds in only one patient for the length (\#6), the diameter tapering (\#13), the radius (\#20), the change in wall thickness (\#27) and the wall thickness (\#34) and the eccentricity ratio (\#68) of the of the basilic, axillary and subclavian vein. 

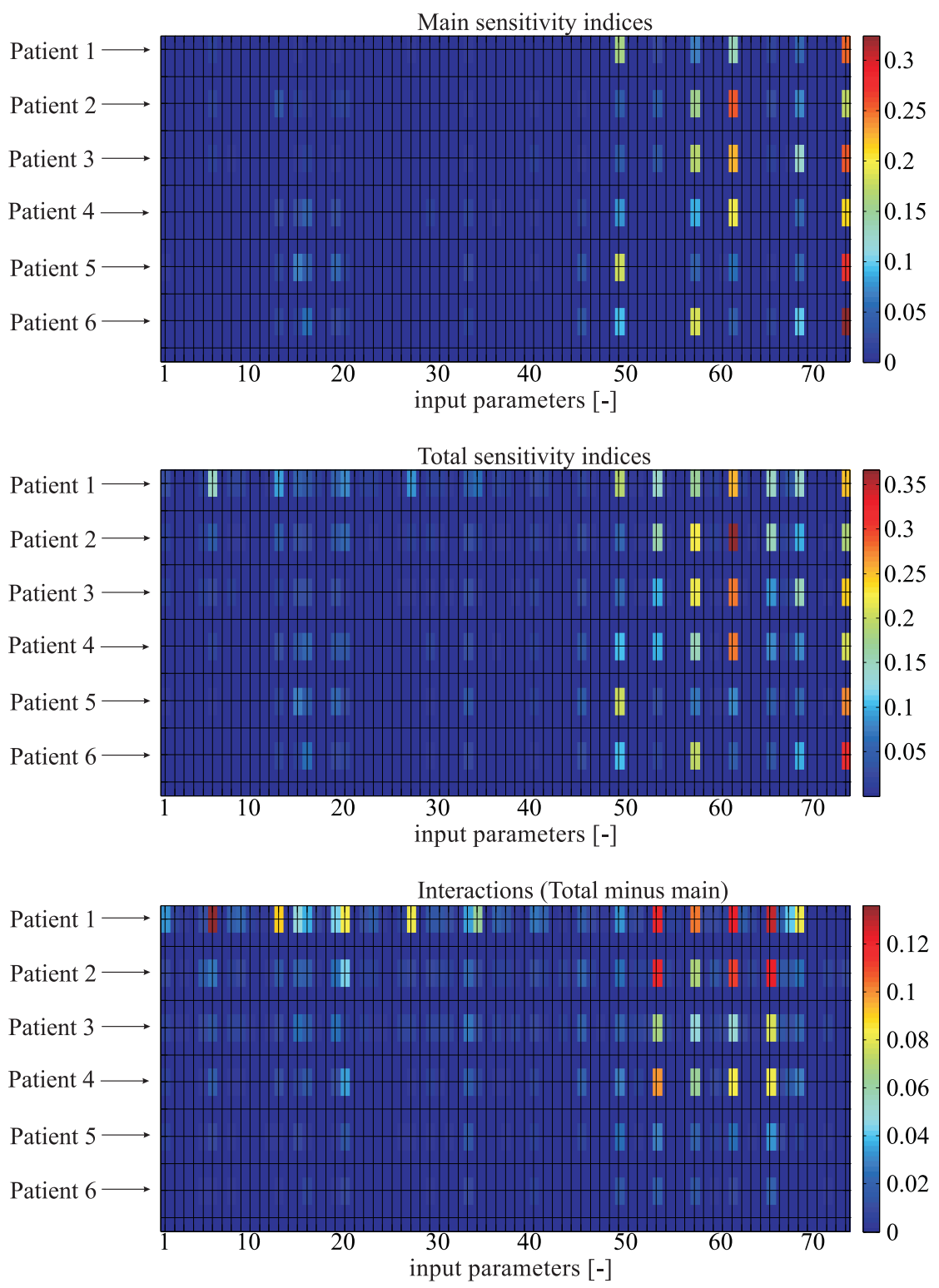

Figure 4.8: The total and main sensitivity indices and their difference for all input parameters with respect to the distal systolic pressure in the upper arm AVF configuration. 


\subsection{Discussion}

In this study we aimed at demonstrating how a sensitivity analysis can help in identifying influential and non-influential model parameters of a distributed lumped parameter pulse wave propagation model, applied to AVF surgery. These parameters are found by using a variance-based method and Monte Carlo simulations. As a result, insight is obtained in how to improve the measurement protocol that is used to determine the input parameters for each patient.

A computational framework has been developed to support the execution of the sensitivity analysis and the management of all data. The framework allows to generate input samples for the Monte Carlo simulations by means of Latin Hypercube sampling, to execute the simulations, to consolidate the data and to calculate the variance-based sensitivity indices using the input and output data. The correct implementation of the framework was checked using the Sobol G-function as simulation model and satisfying results were obtained. After including the wave propagation model into the framework, we determined that 5000 runs per parameter are sufficient to obtain sensitivity indices with an absolute accuracy smaller than 0.05 .

The sensitivity analysis of the wave propagation model was performed for six patient datasets. For each patient two different configurations, i.e. a lower arm AVF and an upper arm AVF, were analyzed and simulated, having respectively 73 and 66 input parameters. Because the mean brachial flow and the distal systolic pressure are the most important clinical parameters, the sensitivity results of these output parameters were presented for both the upper and lower arm configurations.

With respect to the mean brachial flow, the aortic windkessel resistance and the mean aortic flow were identified as model parameters that result in significant reductions in output variance for both AVF configurations and for all six patients (Table 4.3). Efforts to measure these parameters more accurately are therefore rewarding to reduce the uncertainty in the predicted mean brachial flow resulting from uncertainties in model parameters. In addition, the model parameters that are associated with the smallest diameter in the inflow and outflow tract of the AVF had significant main sensitivity indices. This corresponds with clinical practice since small arterial and venous calibers are associated with limited flow enhancement $[8,9,20,21]$ and these are thus preoperatively assessed. Our sensitivity analysis suggests that it might be worthwhile to also include a measure for the aortic windkessel resistance and mean aortic flow in the preoperative work-up of AVF surgery planning. Other model parameters that should be measured more accurately are the radial artery windkessel resistance and compliance, intravenous pressure and distributed flow 
(Table 4.3). A high sensitivity index of the radial artery windkessel compliance for the mean brachial flow was not expected, as its impedance is infinitely large for the mean component of the waveform. However, the radial artery windkessel compliance affects the pressure and flow waveforms proximal and distal to the anastomosis and these flow waveforms are used to calculate the anastomosis resistance. In this way, the radial artery windkessel compliance affects the total resistance of the arterial tree and, consequently, the mean brachial flow. The model parameters described above can also not be fixed within their measurement uncertainty interval because also their total sensitivity indices were significant (Table 4.4). This also holds for the aortic windkessel compliance and the length and wall thickness of the basilic, axillary and subclavian vein. In total thus 18 parameters cannot be fixed it with respect to mean brachial flow, whereas the other 55 (lower arm AVF) or 48 (upper arm AVF) can be fixed. Furthermore, it was observed that windkessel compliances and resistances of the aorta and radial artery are significantly involved in interactions. This likely results from the definition of the windkessel elements, where the compliance and resistance occur as product in the associated differential equation.

The mean aortic flow and the aortic windkessel resistance are also significant model parameters with respect to the distal systolic arterial pressure in both AVF configurations (Table 4.3) as their main sensitivity indices were significant. Furthermore, the aortic characteristic impedance and the radial artery windkessel compliance had significant main sensitivity indices for the distal systolic pressure. The identification of these model parameters can physically be explained as follows; a change in systolic aortic flow which might propagate to the systolic radial artery pressure, while the aortic windkessel resistances determines the mean arterial pressure. The aortic characteristic impedance influences the pressure and flow wave reflections, whereas the radial artery windkessel compliance influences wave reflections from the radial artery periphery. Other model parameters that also had significant main sensitivity indices were the diameters of the AVF inflow tract and eccentricity ratios in the outflow tract. These parameters effect the vessel compliances of the inflow and outflow tract but also the pressure drop over the anastomosis and as a result, the systolic radial artery pressure. For the distal systolic pressure 59 (lower arm AVF) or 51 (upper arm AVF) model parameters can be fixed within their measurement uncertainty interval, whereas respectively 9 or 8 parameters might be rewarding for measurement improvements.

By considering both configurations for both output parameters, in total 16 model parameters should be measured as accurate as possible in order to reduce outcome uncertainty. These and six additional parameters could not be fixed, whereas 51 out of 73 input pa- 
rameters could be fixed within their measurement uncertainty. However as these fixable parameters still need to be measured, a new sensitivity analysis should be performed to determine which parameters can be fixed within the uncertainty range of a generic population. In this new sensitivity analysis the spread observed in the population as a whole should be used to define the uncertainty distributions of the model parameters. The insight obtained in this study gives us the opportunity to further improve our measurement protocol, whereas the next study, will give us insight into which model parameters can be made into a generic constant. The two analyses together provide us with the necessary information on how to adapt the model to patient-specific conditions and enable us to perform a patient-specific analysis with error propagation on a larger patient population. The predicted hemodynamic parameters with uncertainty interval can subsequently be used to perform proper clinical model verification by both qualitatively and quantitatively corroborating the simulation results with clinical observations (i.e. model corroboration). This clinical corroboration studies should indicate if the model is applicable in a clinical setting as it is.

In this sensitivity analysis study, uniform uncertainty distributions were used to select input samples. Other input distribution shapes will change the weight of each input sample. For example in case of a truncated Gaussian, we are more certain about the measured value of the model parameter and therefore the Monte Carlo samples are more densely distributed around the true value of the model parameter than in the case of uniform distribution where the Monte Carlo samples are equally spread over the uncertainty domain. As a result, the total output variance and the quantitative values of the sensitivity indices might differ. However, we observed that the model parameters identified as influential and non-influential were not significantly different with respect to parameter prioritization and fixing when using truncated Gaussian distributions instead of a uniform ones. Therefore, we think that the results of our sensitivity study will not be significantly different and can therefore be used to deliberately improve our measurement protocol. However, future efforts to depict the exact input distributions can be rewarding for a better estimate of the output uncertainty.

For considering the correlations between model parameters in our sensitivity analysis, correlations should be available for a large population group. Since we did not have access to such data, we assumed that all input parameters are independent of each other. Although the interpretation of the main and total sensitivity indices is more difficult for correlated input, these indices can still be used for parameter prioritization and fixing $[68,114]$. However, the Sobol decomposition in (4.4) no longer holds and the multi-dimensional integrals describing the sensitivity indices should be estimated by Monte Carlo simulations 
using a brute force approach and by generating samples from conditional distributions by using for example Markov Chain Monte Carlo [68]. If only a small subset of the parameters in the input space is correlated, the subset can be considered as one combined parameter that is independent of the other parameters. In this case, the method to calculate the sensitivity indices in our study can be applied to correlated input [114]. Other methods that deal with correlated input are the method introduced by Ratto et al. [115] or methods that are based on metamodels, which are approximations of the actual model and derived by defining a simplified relations between the input parameters and the output of interest, e.g. nonparametric regression methods [69, 116, 117, 118].

When a computational model has much larger computational costs than the wave propagation model in our study (e.g. in the case of three-dimensional models), Monte Carlo simulations can become impractical. In such cases, metamodeling can also be a better approach and a sophisticated design ([119]) of the computational experiment is needed.

\subsection{Conclusion}

In summary, we developed a computational framework to perform a sensitivity analysis with a variance-based method and Monte Carlo simulations within a reasonable amount of time. The framework was used to determine which parameters of our distributed lumped parameter wave propagation model for AVF surgery are the most important for a reliable outcome. Of all 73 parameters, 16 parameters were identified which are rewarding to be measured as accurate as possible in order to reduce the uncertainty in the output predictions. The most rewarding model parameters were the aortic windkessel resistance, the mean aortic flow, the parameters associated with the smallest arterial or venous diameters of the AVF in- and outflow tract and the radial artery windkessel compliance.

\section{Appendix: Mathematical background and numerical im- plementation}

\section{Variance-based method}

The main and total sensitivity indices in equation (4.2) and (4.5) consist of multi-dimensional integrals, which are presented in this appendix. These multi-dimensional integrals can be evaluated by Monte Carlo experiments resulting in estimates for the main and total sensitivity indices. This is described in this appendix that is based on Saltelli et al. 
[103] and is added to this article to make this work self-contained and to improve the understanding of the variance-based method. For more details about the method we refer to the paper of Saltelli et al. [103].

\section{Definitions}

The unconditional expectation value and mean of a model $Y=f\left(X_{1}, X_{2}, \ldots, X_{k}\right)$ with all independent random input parameters $X_{i}$ which are defined on the domain $\Omega=\Omega_{1} \times$ $\Omega_{2} \times \ldots \times \Omega_{k}$ with $\left\{\Omega_{i}=\left[a_{1}, a_{2}\right] \mid a_{1}<a_{2} \in \Re\right\}$, can be calculated by [103]

$$
E(Y)=\int_{\Omega}\left(f\left(x_{1}, x_{2}, \ldots, x_{k}\right) \prod_{i=1}^{k}\left(p_{i}\left(x_{i}\right) d x_{i}\right)\right),
$$

and

$$
V(Y)=\int_{\Omega}\left(f\left(x_{1}, x_{2}, \ldots, x_{k}\right)^{2} \prod_{i=1}^{k}\left(p_{i}\left(x_{i}\right) d x_{i}\right)\right)-E^{2}(Y)
$$

respectively. Herein $p_{i}\left(x_{i}\right)$ is the marginal probability function of input parameter $X_{i}$. The conditional variance is obtained by fixing one or more of the input parameters into a constant. If one of the input parameters is fixed to value $x_{i}^{*}$ the conditional variance is defined by

$$
\begin{array}{r}
V\left(Y \mid X_{i}=x_{i}^{*}\right)=\int_{\Omega \backslash \Omega_{i}}\left(f\left(x_{1}, x_{2}, \ldots, x_{i}^{*}, \ldots x_{k}\right)^{2} \prod_{\substack{j=1 \\
j \neq i}}^{k}\left(p_{j}\left(x_{j}\right) d x_{j}\right)\right) \\
-E^{2}\left(Y \mid X_{i}=x_{i}^{*}\right)
\end{array}
$$

in which $\Omega \backslash \Omega_{i}$ indicates the domain $\Omega$ except the domain of $X_{i}$. The expectation value of the conditional variance $E\left(V\left(Y \mid X_{i}=x_{i}^{*}\right)\right)=E\left(V\left(Y \mid X_{i}\right)\right)$ (residual effect) results by integrating the conditional variance, weighted by the probability density function of $X_{i}$, over all possible values of parameter $x_{i}^{*}$, i.e. over the domain $\Omega_{i}$. And is given by

$$
\begin{array}{r}
E\left(V\left(Y \mid X_{i}\right)=\int_{\Omega}\left(f\left(x_{1}, x_{2}, \ldots, x_{i}, \ldots x_{k}\right)^{2} \prod_{j=1}^{k}\left(p_{j}\left(x_{j}\right) d x_{j}\right)\right)\right. \\
-\int_{\Omega_{i}}\left(E^{2}\left(Y \mid X_{i}=x_{i}^{*}\right)\left(p_{i}\left(x_{i}^{*}\right) d x_{i}^{*}\right)\right) .
\end{array}
$$


By using equation (4.1), i.e. subtracting equation (4.9) from the total conditional variance (4.7), the main effect $V\left(E\left(Y \mid X_{i}\right)\right)$ can be derived. This gives

$$
\begin{array}{r}
V(Y)-E\left(V\left(Y \mid X_{i}\right)=V\left(E\left(Y \mid X_{i}\right)\right)=\int_{\Omega_{i}}\left(E^{2}\left(Y \mid X_{i}=x_{i}^{*}\right) p_{i}\left(x_{i}^{*}\right) d x_{i}^{*}\right)\right. \\
-E^{2}(Y) \equiv U_{i}-E^{2}(Y) .
\end{array}
$$

The main sensitivity index $S_{i}=\frac{V\left(E\left(Y \mid X_{i}\right)\right)}{V(Y)}$ can now be expressed by dividing equation (4.10) by (4.7) which results in

$$
S_{i}=\frac{U_{i}-E^{2}(Y)}{V(Y)}
$$

Following an analog derivation as for the main sensitivity index but know by fixing all parameters except $X_{i}$ similar expressions can be derived for the total sensitivity index. The main effect is in this case $V\left(E\left(Y \mid X_{1}=x_{1}^{*}, \ldots, X_{i-1}=x_{i-1}^{*}, X_{i}, X_{i+1}=\right.\right.$ $\left.\left.x_{i+1}^{*}, \ldots, X_{k}=x_{k}^{*}\right)\right) \equiv V\left(E\left(Y \mid X_{-i}\right)\right)$ and is given by

$V\left(E\left(Y \mid X_{-i}\right)\right)=\int_{\Omega \backslash \Omega_{i}}\left(E^{2}\left(Y \mid X_{-i}\right) \prod_{\substack{j=1 \\ j \neq i}}^{k}\left(p_{j}\left(x_{j}\right) d x_{j}\right)\right)-E^{2}(Y) \equiv U_{-i}-E^{2}(Y)$

From equation (4.1) we know that $E\left(V\left(Y \mid X_{-i}\right)\right)=V(Y)-V\left(E\left(Y \mid X_{-i}\right)\right)=V(Y)-$ $\left(U_{-i}-E^{2}(Y)\right)$. From this and equation (4.12) an expression for the total sensitivity index $S_{T_{i}}=\frac{E\left(V\left(Y \mid X_{-i}\right)\right)}{V(Y)}$ can be derived, i.e.:

$$
S_{T_{i}}=1-\frac{\left(U_{-i}-E^{2}(Y)\right)}{V(Y)}
$$

The main and total sensitivity indices in (4.11) and (4.13) can be calculated by evaluating the multi-dimensional integrals in equation (4.7), (4.10) and (4.12) by means of a Monte Carlo experiment. This is described in the next section of this appendix and also based on Saltelli et al. [103]. 


\section{Estimation of the sensitivity indices}

Ishigami et al. [120] showed by using $E^{2}\left(Y \mid X_{i}\right)=\left(E\left(Y \mid X_{i}\right)\right)^{2}$ that the multi-dimensional integral $U_{i}=\int_{\Omega_{i}}\left(E^{2}\left(Y \mid X_{i}=x_{i}^{*}\right) p_{i}\left(x_{i}^{*}\right) d x_{i}^{*}\right)$ can be rewritten as

$$
\begin{aligned}
U_{i}=\int_{\Omega}\left(f\left(x_{1}, x_{2}, \ldots, x_{i}, \ldots, x_{k}\right) f\left(x_{1}^{\prime}, x_{2}^{\prime}, \ldots, x_{i}, \ldots, x_{k}^{\prime}\right) .\right. \\
\left.\prod_{j=1}^{k}\left(p_{j}\left(x_{j}\right) d x_{j}\right) \prod_{\substack{j=1 \\
j \neq i}}^{k}\left(p_{j}\left(x_{j}^{\prime}\right) d x_{j}^{\prime}\right)\right) .
\end{aligned}
$$

Herein the primed variables are introduced to better understand the estimations of the sensitivity indices by means of the Monte Carlo experiment. Namely, realize that equation (4.14) is the expectation value of the function

$$
\begin{aligned}
F\left(x_{1}, \ldots, x_{k}, \ldots, x_{1}^{\prime}, \ldots, x_{i-1}^{\prime}, x_{i+1}^{\prime}, \ldots, x_{k}^{\prime}\right) & \\
& =f\left(x_{1}, \ldots, x_{k}\right) f\left(x_{1}^{\prime}, \ldots, x_{i-1}^{\prime}, x_{i}, x_{i+1}^{\prime}, \ldots, x_{k}^{\prime}\right)
\end{aligned}
$$

with $2 k-1$ model parameters. Therefore the integral in (4.14) be calculated by a single Monte Carlo loop [103].

A comparable derivation can be made for $U_{-i}$ resulting in

$$
\begin{array}{r}
U_{-i}=\int_{\Omega}\left(f\left(x_{1}, x_{2}, \ldots, x_{i}, \ldots, x_{k}\right) f\left(x_{1}, x_{2}, \ldots, x_{i}^{\prime}, \ldots, x_{k}\right)\right. \\
\left.\prod_{j=1}^{k}\left(p_{j}\left(x_{j}\right) d x_{j}\right)\left(p_{j}\left(x_{j}^{\prime}\right) d x_{j}^{\prime}\right)\right)
\end{array}
$$

which is the expectation value of the function

$$
K\left(x_{1}, \ldots, x_{k}, x_{i}^{\prime}\right)=f\left(x_{1}, \ldots, x_{i}, \ldots, x_{k}\right) f\left(x_{1}, \ldots, x_{i}^{\prime}, \ldots, x_{k}\right)
$$

with $k+1$ model parameters.

To estimate the main and total sensitivity indices in (4.11) and (4.13) estimates for $U_{i}$, $U_{-i}, V(Y)$ and $E^{2}(Y)$ are required resulting in

$$
\hat{S}_{i}=\frac{V\left(E\left(Y \mid X_{i}\right)\right)}{V(Y)} \approx \frac{\hat{U}_{i}-\hat{E}^{2}(Y)}{\hat{V}(Y)}
$$


and

$$
\hat{S}_{T_{i}}=\frac{E\left(V\left(Y \mid X_{-i}\right)\right)}{V(Y)} \approx 1-\frac{\hat{U}_{-i}-\hat{E}^{2}(Y)}{\hat{V}(Y)}
$$

respectively. To compute these estimates, two Monte Carlo input sample matrices with dimension $N \times k$ are generated:

$$
\mathbf{M}_{\mathbf{1}}=\left(\begin{array}{ccc}
x_{1}{ }^{(1)} & \ldots & x_{k}{ }^{(1)} \\
x_{1}{ }^{(2)} & \ldots & x_{k}{ }^{(2)} \\
\vdots & \vdots & \vdots \\
x_{1}{ }^{(N-1)} & \ldots & x_{k}^{(N-1)} \\
x_{1}{ }^{(N)} & \ldots & x_{k}{ }^{(N)}
\end{array}\right) \quad, \quad \mathbf{M}_{2}=\left(\begin{array}{ccc}
x_{1}^{\prime(1)} & \ldots & x_{k}^{\prime(1)} \\
x_{1}^{\prime(2)} & \ldots & x_{k}^{\prime(2)} \\
\vdots & \vdots & \vdots \\
x_{1}^{\prime(N-1)} & \ldots & x_{k}^{\prime(N-1)} \\
x_{1}^{\prime(N)} & \ldots & x_{k}^{\prime(N)}
\end{array}\right)
$$

where $N$ is the sample size used for the sensitivity estimates and $k$ is the number of parameters. Matrix $\mathbf{M}_{1}$ is called the sample matrix and matrix $\mathbf{M}_{2}$ the re-sample matrix. Next, two matrices are determined for each parameter $X_{i}$. The first matrix $\mathbf{M}_{\mathbf{S}_{\mathbf{i}}}$ is the re-sample matrix in which the $i^{t h}$-column is replaced by the $i^{t h}$-column of the sample matrix. Thus compared to the sample matrix all samples of the input parameters are changed except the samples of parameter $X_{i}$ which is fixed. Matrix $\mathbf{M}_{\mathbf{S}_{\mathbf{i}}}$ will be used to estimate the main sensitivity index. The second matrix $\mathbf{M}_{\mathbf{S}_{\mathbf{T}_{\mathbf{i}}}}$ is the sample matrix in which only the $i^{t h}$-column is replaced by the $i^{t h}$-column of the re-sample matrix, i.e. all input parameters except $X_{i}$ are fixed. Matrix $\mathbf{M}_{\mathbf{S}_{\mathbf{T}_{\mathbf{i}}}}$ will be used to estimate the total sensitivity index. Matrices $\mathbf{M}_{\mathbf{1}}, \mathbf{M}_{\mathbf{2}}, \mathbf{M}_{\mathbf{S}_{\mathbf{i}}}$ and $\mathbf{M}_{\mathbf{S}_{\mathbf{T}_{\mathbf{i}}}}$ are now used to run our model $Y=$ $f\left(x_{1}^{l}, \ldots, x_{k}^{l}\right)$ with $l=1,2, \ldots, N-1, N$. This results in estimates for the output vectors $\mathbf{Y}_{\mathbf{1}}=f\left(\mathbf{M}_{\mathbf{1}}\right), \mathbf{Y}_{\mathbf{2}}=f\left(\mathbf{M}_{\mathbf{2}}\right), \mathbf{Y}_{\mathbf{S}_{\mathbf{i}}}=f\left(\mathbf{M}_{\mathbf{S}_{\mathbf{i}}}\right)$ and $\mathbf{Y}_{\mathbf{T}_{\mathbf{i}}}=f\left(\mathbf{M}_{\mathbf{S}_{\mathbf{T}_{\mathbf{i}}}}\right)$. The parameters $\hat{U}_{i}$ and $\hat{U}_{-i}$ in equations (4.16) and (4.17) are computed from the scalar products of the above defined vectors of model outputs, i.e. [103]:

$$
\hat{U}_{i}=\frac{1}{N-1} \sum_{j=1}^{N} y_{1}^{j} y_{S_{i}}^{j} \quad \text { and } \quad \hat{U}_{-i}=\frac{1}{N-1} \sum_{j=1}^{N} y_{1}^{j} y_{S_{T_{i}}}^{j}
$$

in which $j$ represents the output of the $j^{\text {th }}$ model run for each output vector. The estimated total variance $\hat{V}(Y)$ can be obtained from either $\mathbf{Y}_{\mathbf{1}}$ or $\mathbf{Y}_{\mathbf{2}}$. In our framework, it is calculated from $\mathbf{Y}_{\mathbf{1}}=f\left(\mathbf{M}_{\mathbf{1}}\right)$ for both the estimations of the main and total sensitivity 
index [103]:

$$
\hat{V}(Y)=\frac{1}{N} \sum_{j=1}^{N} y_{1}^{j} y_{1}^{j}-\left(\frac{1}{N} \sum_{j=1}^{N} y_{1}^{j}\right)^{2} .
$$

For the main sensitivity index $\hat{E}^{2}(Y)$ is estimated by [103]:

$$
\hat{E}^{2}(Y) \approx \frac{1}{N} \sum_{j=1}^{N} y_{1}^{j} y_{2}^{j}
$$

whereas in the case of the total sensitivity index $\hat{E}^{2}(Y)$ is approximated by

$$
\hat{E^{2}}(Y) \approx\left(\frac{1}{N} \sum_{j=1}^{N} y_{1}\right)^{2}
$$

These choices for $\hat{E}^{2}(Y)$ result in better estimates of the main and total sensitivity index as is given by $[103,106]$.

The 95\%-confidence interval of the main and total sensitivity indices are determined by applying bootstrapping [112]. In bootstrapping, the output vectors $\mathbf{Y}_{\mathbf{S}_{\mathbf{i}}}$ and $\mathbf{Y}_{\mathbf{T}_{\mathbf{i}}}$ are changed by permutation. Every permutation the sensitivity indices are calculated by using the permutated output vectors in equation (4.18), resulting in new estimates for the sensitivity indices. In this study we performed 100 permutations which was sufficient to reach convergence for the $95 \%$-confidence interval.

\section{Convergence check}

In this appendix the procedure is described to determine the number of parameters runs that are needed to reach convergence of the sensitivity estimates with our computing framework.

\section{Methods}

Monte Carlo simulations are executed for $\mathrm{N}=250,500,1000,2000,3000,4000,5000$, 7500 and 10000 runs per parameter. After each run we carried out bootstrapping (100 times) to determine the $95 \%$-confidence interval. 


\section{Results and conclusion}

As an example Figure 4.9 shows the total sensitivity indices for the aortic characteristic impedance and the aortic peripheral resistance with respect to the mean brachial flow in a lower arm AVF as function of the number of Monte Carlo runs per parameter. Similar trends are observed for all other sensitivity indices between input and output parameters. Increasing the number of runs per parameter reduces the $95 \%$-confidence interval resulting in more precise estimates. Furthermore, it can be seen that the $95 \%$-confidence intervals for the sensitivity index overlap each other independent of the number of Monte Carlo runs. For all sensitivity indices the minimum number of model runs per parameter is determined for which the sensitivity estimate and the $95 \%$-confidence interval remain within 0.05 compared to the weighted average for all Monte Carlo experiments. For higher sensitivity values more runs per input parameter are needed to obtain convergence. For our study, in which we defined mean brachial flow and the systolic pressure distal to the anastomosis as output of interest, at least 3000 model runs per parameter are needed. In this study, we used 5000 model runs per parameter and the sensitivity indices are thus accurate within \pm 0.05 .

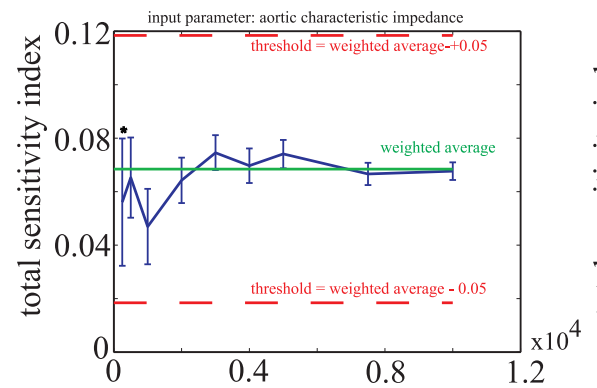

Number of runs per parameter [-]

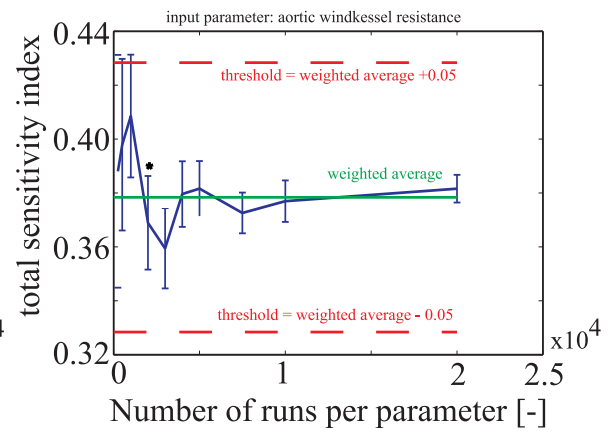

Number of runs per parameter [-]

* From here all total indices and their $95 \%$ confidence interval remain within the threshold.

Figure 4.9: Estimates of the total sensitivity indices as function of the number of runs per parameter for the aortic characteristic impedance (left) and the aortic windkessel resistance (right) with respect to the mean brachial flow. 


\section{Benchmark model: the Sobol G-function}

\section{Methods}

To validate if the sensitivity indices are correctly estimated by our computational framework, sensitivity indices were calculated in a benchmark model for which these indices are known from analytical theory: the Sobol G-function. The Sobol G-function is a strongly non-monotonic, non-additive function of $k$ parameters $x_{i}$, that are assumed to be identically and uniformly distributed in the unit hypercube $\left\{I^{k}=x \mid 0 \leq x_{i} \leq 1 ; i=1,2, \ldots, k\right\}$. It is often used in literature to benchmark the calculation of sensitivity indices [75, 103, 112, 113]. The Sobol G-function is given by [75]

$$
G\left(x_{1}, \ldots, x_{k}, a_{1}, \ldots, a_{k}\right)=\prod_{i=1}^{k} g_{i}\left(x_{i}, a_{i}\right) \quad \text { with } \quad g_{i}\left(x_{i}, a_{i}\right)=\frac{\left|4 x_{i}-2\right|+a_{i}}{1+a_{i}}
$$

For each of the $g_{i}\left(x_{i}, a_{i}\right)$ functions $\int_{0}^{1} g_{i}\left(x_{i}, a_{i}\right) d x_{i}=1$, and for $x_{i} \in[0,1]$ the variation of the function is

$$
1-\frac{1}{1+a_{i}} \leq g_{i}\left(x_{i}, a_{i}\right) \leq 1+\frac{1}{1+a_{i}} .
$$

The importance of each parameter $x_{i}$ is thus determined by the coefficient $a_{i}$ which is a positive real number. In Table 4.6 the input parameters and the corresponding coefficients $a_{i}$ that are used for the benchmark model are given. Coefficients $a_{i}$ are selected such that also interactions are present within the model. The benchmark analysis is performed for 5000 and for 10000 runs per parameter and bootstrapping (100 permutations) is applied to determine the $95 \%$-confidence interval.

The main sensitivity indices can be obtained analytically from [75]

$$
S_{i}=\frac{V_{i}}{V(Y)}=\frac{V\left(E\left(Y \mid X_{i}\right)\right)}{V(Y)}=\frac{1}{V(Y) 3\left(1+a_{i}\right)^{2}} \quad \text { with } \quad V(Y)=\prod_{i=1}^{k}\left(1+V_{i}\right)-1
$$

whereas the total sensitivity index is analytically expressed by [75]

$$
S_{T i}=\frac{E\left(V\left(Y \mid X_{-i}\right)\right)}{V(Y)}=\frac{V_{i}}{V(Y)} \prod_{j \neq i}\left(1+V_{j}\right) \quad \text { with } \quad V(Y)=\prod_{i=1}^{k}\left(1+V_{i}\right)-1
$$


Table 4.6: Definition of the Sobol G-function (4.22).

$\begin{array}{ccc}\text { Parameter name(s) [-] } & a_{i} \text { value [-] } & \text { Remarks } \\ X_{1} & 0 & \text { Most important first order effect } \\ X_{2} & 0.5 & \\ X_{3} & 1 & \\ X_{4} & 2 & \\ X_{5} & 4.5 & \\ X_{6}, X_{7}, X_{8} & 9 & \text { Non-important first-order effects } \\ X_{9} \text { till } X_{15} & 99 & \text { Non-influential parameters at all }\end{array}$

\section{Results and conclusion}

Results of the benchmarking are presented in Figure 4.10. For the most influential parameters a very small difference (maximum 0.05 ) between the analytical solution and the estimated indices can be observed, whereas for the least influential parameters the sensitivity estimates equal the analytical sensitivity indices. Also in the case of interactions (note large difference between main and total sensitivity index), it can be clearly observed for the three most important model parameters that the correct estimates are obtained. Increasing the number of runs from 5000 to 10000 per parameter does not influence the results. The developed framework is thus able to correctly calculate sensitivity indices. 

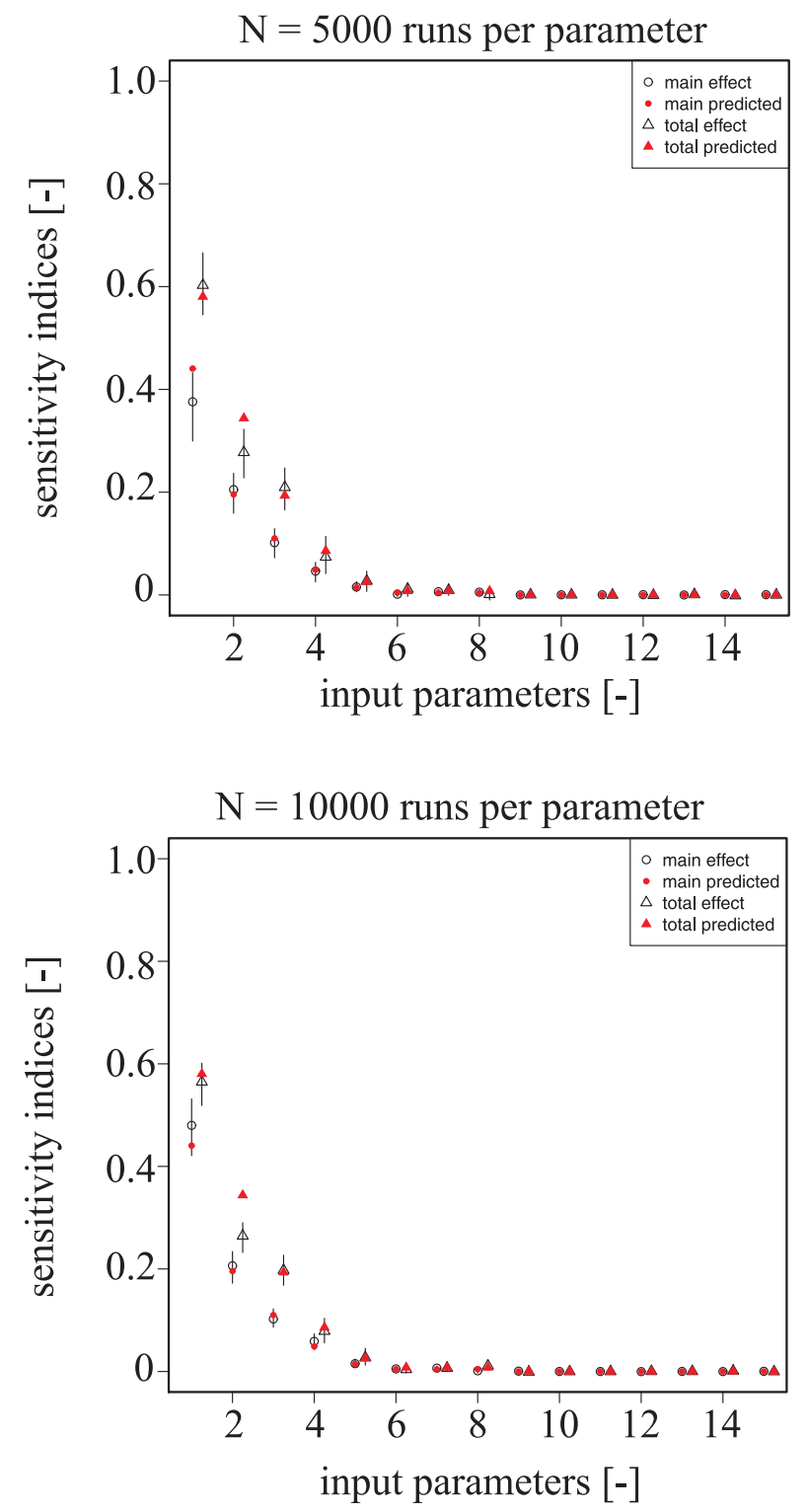

Figure 4.10: The total and main sensitivity indices calculated for the Sobol G-function both with our framework (black) and analytically (red). At the top the benchmark model simulated with 5000 runs per parameter is presented, whereas at the bottom the benchmarking with 10000 runs per parameter is shown. 


\section{Chapter 5}

\section{A sensitivity analysis to reduce the number of patient-specific inputs}

This Chapter is based on:

W. Huberts, C. de Jonge, W.P.M. van der Linden, M.A. Inda, J.H.M. Tordoir, F.N. van de Vosse, E.M.H. Bosboom, A sensitivity analysis to reduce the number of input parameters in a personalized pulse wave propagation model for AVF surgery, Submitted, (2011) 
114 Chapter 5. A sensitivity analysis to reduce the number of patient-specific inputs

\begin{abstract}
Decision-making in vascular access surgery for hemodialysis can be supported by a pulse wave propagation model. To personalize such a model, patient-specific input parameters should be chosen. However, the number of input parameters that can be measured in clinical routine is limited. In addition, patient data are compromised with uncertainty. Incomplete and uncertain input data will result in uncertain model output. Previously, we analyzed the propagation of measurement uncertainty in the input to the uncertainty in the model output by means of a sensitivity analysis. Of all 73 parameters, 51 could be fixed within their measurement uncertainty range. But these parameters still needed to be measured. To reduce the number of measurements, we present a methodology for assessing those model input parameters that can be taken constant. In addition, a method to determine this constant is presented. For the pulse wave propagation model applied to vascular access surgery, six patient-specific datasets were analyzed and 47 out of 73 parameters can be fixed on a generic value. These model parameters are less important when personalizing the wave propagation model. Furthermore, we were able to determine generic values for 37 of the 47 fixable model parameters.
\end{abstract}




\subsection{Introduction}

For treatment of end-stage renal disease (ESRD) patients by means of hemodialysis therapy, a vascular access (VA) is required. The vascular access is the site at the body where blood is withdrawn to the dialyzer and returned after filtration. The blood flow rate in a VA should be at least $600 \mathrm{ml} / \mathrm{min}$ and, in addition, the vascular access should be easily accessible for repeated cannulation over time [8,9]. Because there is no vessel in the human body which complies with these requirements, a vascular access is surgically created. An arteriovenous fistula (AVF) in the arm, which is a surgically created permanent connection between an artery and vein, is the preferred option, as it has a better long-term patency and lower complications rates compared to other vascular access types like grafts and catheters $[8,9]$. By creating an AVF, the peripheral resistance in the hand is bypassed, which results in vessel dilatation and a ten- to thirtyfold increase in blood flow rate. The location of the AVF in the arm is determined by the vascular surgeon based on preoperative diagnostics and clinical experience. However, lower arm AVFs are hampered by non-maturation in up to $50 \%$ of all cases $[6,7,10]$, which implies that six weeks after surgery either the blood flow rate is not increased sufficiently ( $<600 \mathrm{ml} / \mathrm{min}$ ), and/or the venous diameter is not sufficiently dilated $(<6 \mathrm{~mm})$ and/or the VA is located too deep $(>6 \mathrm{~mm})$. Upper arm AVFs do not suffer from short-term complications like non-maturation, but they are compromised by high flow rate problems like cardiac failure and distal ischemia in up to $20 \%$ of all cases $[7,8,12,13,14]$. These upper arm AVF complications predominantly occur, when the vascular access flow is larger than $30 \%$ of the cardiac output $[12,13,14]$, whereas non-maturation is associated with a low direct postoperative flow $[12,32]$. A low systolic pressure distal to the anastomosis is indicative for distal ischemia $[7,8,9]$.

Vascular access planning by the surgeon could be improved when before surgery a quantitative measure of both the vascular access flow (brachial artery inflow) and the systolic pressure distal to the anastomosis were available. Therefore, previously, we developed a distributed lumped parameter pulse wave propagation model, that can make an individualized prediction of the postoperative mean brachial flow and the systolic pressure distal to the anastomosis [101].

To use distributed wave propagation models in individualized treatment planning, model input parameters should be adapted to patient-specific conditions and therefore measurements on patients are needed. However, in clinical practice, measurements are associated with relatively large measurement uncertainties compared to in-vitro measurements resulting from measurement time, limited facilities and available modalities. Very often 
only one measurement is performed. In addition, biological variations are large, which adds up to the measurement uncertainty. Hence, it is impossible to access all model input parameters patient-specifically and sufficiently accurately, resulting in sparse datasets comprised by large uncertainties. These uncertain and incomplete datasets will result in output uncertainties and insight into the propagation of measurement uncertainties to output uncertainty is needed. This insight can be obtained by a sensitivity analysis, in which the output uncertainty is apportioned to input parameters uncertainty $[68,69,121]$.

Previously, we performed a global sensitivity analysis on our wave propagation model to examine which parameters should be measured more accurately to reduce the uncertainty in the prediction of mean brachial flow and the systolic distal radial artery pressure in [121]. We found that improving measurements for only 16 out of 73 model parameters was rewarding and 51 parameters could be fixed within their measurement uncertainty interval. However, in this case these 51 parameters still need to be measured or estimated for each patient. To reduce the number of measurements, we aim to present a methodology to identify model input parameters, for which a constant generic value can be taken (parameter fixing).

An intuitive strategy to determine which model parameters can be fixed is to first define model parameters for an average patient, and thereafter, perform a sensitivity analysis in which all model parameters are varied within their population uncertainty domain. However, defining parameters for an average patient is not trivial, because for each parameter many patient data are required to know the mean value. In addition, model parameters might be grouped within the complete input space, for example as a result of gender differences or because of a relation between parameter and age or body-mass-index (BMI). As a result, a sensitivity analysis for parameter fixing should be performed for average patients for all groups to avoid non-physiological combinations of input parameters since in a sensitivity analysis all model parameters are varied within the complete population uncertainty domain. For non-physiological parameter combinations, the model will not converge and this will hamper the sensitivity analysis. Therefore, in this study we chose a different approach. We performed a sensitivity analysis by using patient-specific datasets and applied the population uncertainty on each individual set. This approach will reduce the chance of non-physiological input while the relevant part of the input space is still covered. In this study, we will present our methodology and apply it to six patient-specific datasets.

The outline of this paper is as follows. First, we will shortly describe our distributed wave propagation model and the global sensitivity method that is used in this study and that is captured in our previously developed framework [121]. Thereafter, we will de- 
scribe the input parameters that are selected as possible candidates for parameters fixing and we will define their uncertainty domains based on population spreads. Furthermore, the Monte Carlo experiments executed for the global sensitivity analysis are described. Finally, the parameters are discussed that can be fixed and generically chosen.

\subsection{Material and methods}

\subsubsection{The wave propagation model}

The wave propagation model used in this study, has demonstrated to have potential in supporting AVF surgery planning [101]. For this model, the vascular tree is divided in segments that are connected based on anatomy (Figure 5.1). In each segment the relation between local pressure and flow is described. To model the effect of vascular access creation on blood pressure and flow distribution, segments are needed that represent arteries, veins and the anastomosis (Figure 5.1). All arterial and venous segments are modeled with a lumped parameter approach derived from local mass and momentum equations. The anastomosis segment is modeled with a nonlinear resistor that amongst others depends on anastomosis angle and blood flow. Parts of the cardiovascular system for which information on local pressure and flow is not needed, are truncated and terminated with three-element windkessels. Assembling all lumped segments results in a system of differential equations that describes the pulse wave propagation of the pressure and flow waveforms. This system is solved by numerical integration applying the trapezium rule for implicit time integration [101]. On the first node a measured aortic flow is prescribed, whereas the vessel at venous outflow is closed with a fixed intravenous pressure. For more details about the model we refer to Huberts et al. [101].

In this study we use two different model topologies (computational domains), which are shown in Figure 5.1. The first topology represents a lower arm AVF configuration and consists of the main arteries of the right arm, i.e. the brachiocephalic, subclavian, axillary, brachial, interosseus, radial and ulnar artery. In addition, a truncated part of the aorta, the vertebral artery and the right and left common carotid arteries are included. To model the AVF, this topology is further extended with the anastomosis segment and a venous outflow tract that consists here of the cephalic vein in the upper and lower arm, the median cubital vein, the basilic vein in the upper arm and the subclavian vein. The second topology represents an upper arm AVF. The outflow tract now consists of the basilic, axillary and subclavian vein. 

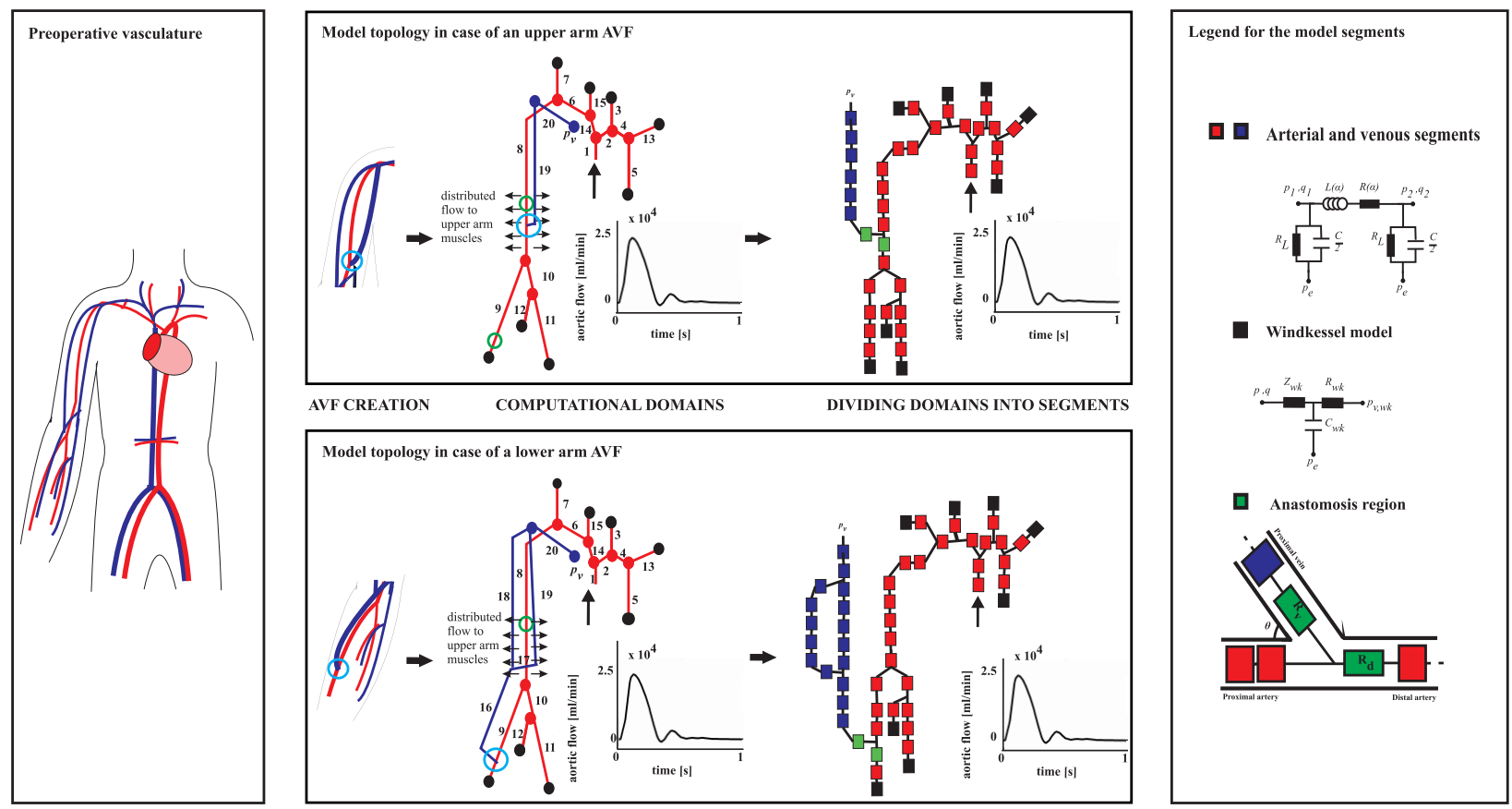

Figure 5.1: A schematic picture of the torso and arm vasculature including the major arteries and the superficial veins (left). The procedure of creation of an arteriovenous fistula (permanent connection between the artery and the vein) in the upper (left, top) and lower (left, bottom) arm are shown. Furthermore, the computational domains and how these domains are divided into lumped parameter segments in case of an upper arm AVF (top) and a lower arm AVF (bottom) are shown [101]. The blue circles on the computational domains indicate the anastomosis locations while the green circles represent the output locations. The edge number refer to the arteries and veins given in Table 5.1 
Table 5.1: The edge numbers and their names.
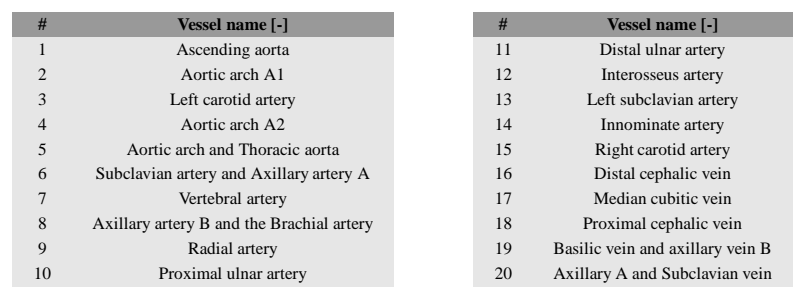

\subsubsection{Global sensitivity analysis}

The global sensitivity analysis used in this study is a variance-based method that was previously described by Saltelli et al. $[68,69,103]$ and applied to our pulse wave propagation model in [121] to identify model parameters that should be measured more accurately to reduce output variance.

\section{Variance-based method}

The variance-based method is a method in which the total output variance $V(Y)$ is apportioned to the input parameters. Sobol [105] showed that for $k$ independent input parameters the relative output variance can be decomposed to

$$
\sum_{i} S_{i}+\sum_{i} \sum_{i>j} S_{i j}+\sum_{i} \sum_{i>j} \sum_{l>j} S_{i j l}+\ldots+S_{12 \ldots k}=1
$$

in which $S_{i} \in[0,1]$ represents the fraction of the total output variance resulting from parameter $X_{i}$ (main sensitivity index), whereas the other indices describe the fractions of the total variance resulting from interactions between parameters. The main sensitivity index is defined as $S_{i}=\frac{\left.V\left(E \mid Y=X_{i}\right)\right)}{V(Y)}$ in which $\left(E \mid Y=X_{i}\right)$ represents the conditional expected value of the output $Y$ and $V\left(E \mid Y=X_{i}\right)$ the variance of this conditional expected value. This sensitivity index can be interpreted as the expected reduction of output variance if the true value of parameter $X_{i}$ would be known and the model parameter is set to its true value. The main sensitivity index can therefore be used to identify which model parameters should be measured more accurately to reduce the output variance. In the case of additive models the total output variance only consists of main effects of the parameters $\left(\sum_{i} S_{i}=1\right)$. However, for non-additive models, in which interactions between the model parameters are present, the main sensitivity index is not sufficient to 
determine which parameter can be fixed within its uncertainty range, because it is possible that the main effect of a parameter will be very low, while its contribution via interaction with other parameters is large. Therefore, also higher order effects should be taken into account, when choosing which model parameters can be fixed within their uncertainty range. However, calculating all higher order effects will be computational expensive unless (5.1) converges to one quickly. Therefore, Homma et al. [106] derived the total sensitivity index for parameter fixing.

A measure for the total sensitivity index is obtained, by first determining the main sensitivity index if all parameters except $X_{i}$ are fixed, i.e.:

$$
S_{-i}=\frac{V_{X_{-i}}\left(E_{X_{i}}\left(Y \mid X_{-i}\right)\right)}{V(Y)},
$$

in which the index $X_{-i}$ represents everything except $X_{i}$. For notational simplicity, we will neglect from this point the indices that define the domain over which the integrals for $E$ and $V$ are evaluated. Note that the main sensitivity index in (5.2) includes all terms in the development (5.1) that do not contain parameter $X_{i}$. Hence $1-\frac{V\left(E\left(Y \mid X_{-i}\right)\right)}{V(Y)}$ equals the sum of all terms (both first order and higher order interactions terms) in (5.1) that do include $X_{i}$. By using $V(Y)=E\left(V\left(Y \mid X_{i}\right)\right)+V\left(E\left(Y \mid X_{i}\right)\right)$ [104], this will result in a total sensitivity index

$$
S_{T_{i}}=\frac{E\left(V\left(Y \mid X_{-i}\right)\right)}{V(Y)},
$$

which is large (close to 1 ) if parameter $X_{i}$ is influential and 0 if the parameter is not influential. The total sensitivity index is, per definition, the expected residual variance that is left, if for all parameters but $X_{i}$, the true value is known. The total sensitivity index is used in this study to determine which parameter is non-influential and can be fixed within its uncertainty range (parameter fixing).

\section{Estimation of the total sensitivity indices}

The total sensitivity indices can be estimated in an efficient way by using Monte Carlo simulations as described in Saltelli et al. [103] and applied to the wave propagation model in our previous study [121]. For the Monte Carlo experiment $N$ random input samples are generated by sampling the marginal probability distributions by means of stratified Latin Hypercube sampling [111]; The input space of each input parameter is divided in $N$ equally probable subspaces and within each subspace a sample is randomly selected 
so that there is only one sample in each subspace. Executing the model now for all $N$ samples will result in the output of interest for each model run, i.e. the mean brachial flow and the radial artery systolic pressure distal to the anastomosis (Figure 5.1). The Monte Carlo experiment is performed with 5000 samples per parameter which has been proven to be a sufficiently large sample size with respect to convergence of the total sensitivity index with a minimal precision of 0.05 [121]. The complete sensitivity analysis was performed with our previously developed framework that is elaborated in Huberts et al. [121].

\subsubsection{Input parameters and analysis}

In this subsection, we describe the model parameters and their uncertainty domains. In addition, a methodology is presented to identify those model parameters for which a generic value can be used.

\section{Definition of patient uncertainty domains}

In analog to our previous study [121], for each edge the following model parameters are taken as input parameters: length, diameter, diameter tapering, wall thickness, wall thickness tapering and Young's modulus. We decided to change the input parameters of predefined edges simultaneously to avoid non-physiological outputs due to large discontinuities between edges (Table 5.2). Other input parameters are the distributed flow through small side-branches in the arm and the mean brachial pressure $\bar{p}_{b}$. Truncated arteries are closed by windkessels, resulting in three input parameters at each arterial end, i.e. the peripheral resistance, compliance and characteristic impedance of the peripheral bed. The veins are closed by prescribing an intravenous pressure. The last input parameter is the aortic flow that is prescribed at the first node.

The uncertainty domains of the input parameters in our previous sensitivity study [121] were based on measurement uncertainties. In this study, the uncertainty domains are broadened and based on the population spread. In this way, when a parameter can be fixed within its domain, measurements of these parameter are no longer required. Not for all parameters the uncertainty domain was broadened, but only for those 51 out of 73 parameters that in our first sensitivity analysis [121] had a total sensitivity index smaller than 0.05 for the mean brachial flow and distal systolic pressure in either of the patients. For all other model parameters the uncertainty domain given in Huberts et al. [121] was kept. An overview of the complete input parameter space and the input uncertainties are shown in Table 5.2. In the remainder of this manuscript these parameters are referred to by using \#. In analog to our previous study [121] uniform distributions are assumed for 
the parameters in this study. The extended uncertainty domains are based on population spreads derived from the OpenClinica database that is created during the $\mathrm{ARCH}$ project (FP7 ICT-224390), which aims to develop computational tools for predicting the clinical outcome of AVF creation [122]. All patients included gave their written informed consent and the study was approved by the Medical ethical committee of Maastricht University Medical Center (The Netherlands) and Bergamo Hospital (Italy). If clinical measurements are not available, the population spread is based on literature.

Arterial and venous lengths. The arterial lengths of the subclavian, axillary and brachial artery (\#1), the radial artery (\#2), the ulnar artery (\#3), the interosseus artery (\#4) and the aorta (\#5) are identified [121] as parameters that can be fixed within their measurement uncertainty. In addition, the length of the cephalic, axillary and brachial vein (\#7) can be fixed. For this a measurement uncertainty interval of $10 \%$ was used based on the assumption that vessel length is proportional to body height [121], which could vary between 153 and $193 \mathrm{~cm}$. In this study, we broadened this to $20 \%$ to also include outliers.

Arterial diameters. From the previous study, the ulnar (\#17) and interosseus (\#18) artery diameters could be fixed within the measurement uncertainty domain of $\pm 10 \%$ [121]. By analyzing data of ninety ESRD patients stored in OpenClinica, a population spread in the ulnar artery diameter, measured by duplex ultrasound was found, of approximately $30 \%$. For the interosseus artery, no data was available, so in analog to the ulnar artery a maximum variation of $30 \%$ in diameter was assumed.

Arterial diameter tapering. Previously, the tapering of the arterial diameters in the subclavian, axillary and brachial artery (\#8), the ulnar artery (\#10), the interosseus artery (\#11) and the aorta (\#12) were identified as parameters that can be fixed within their measurement uncertainty [121]. Within OpenClinica, population spreads for the diameters of the subclavian, axillary, brachial, ulnar artery and aorta of, respectively, 20\% ( $\mathrm{N}=29$ patients), $20 \%(\mathrm{~N}=94), 30 \%(\mathrm{~N}=90)$ and $9 \%(\mathrm{~N}=9)$ were found. These population spreads were used as the maximum variations for diameter tapering in this study, except for the aorta, because more variation is expected in a larger population. Therefore, for aortic diameter tapering $15 \%$ is taken. For diameter tapering in the interosseus artery the same maximum variation as for the ulnar artery is assumed.

Wall thickness and wall thickness tapering. The wall thickness of the subclavian, axillary and brachial artery (\#29), the radial artery (\#30), the ulnar artery (\#31), the in- 
terosseus artery (\#32) and the aorta (\#33) were previously found as parameters that could be fixed within their measurement uncertainty domain of $\pm 40 \%$ [121]. In addition, also the cephalic, axillary and subclavian vein (\#35) wall thickness could be fixed. Within the ARCH project, intima-media thickness measurements were performed on eleven ESRD patients of our hospital in the brachial artery and an intersubject variation of approximately $25 \%$ was found (unpublished data). Konings et al. [123] performed intima-media thickness measurements on ESRD patients in the common carotid artery and observed a variation of $18 \%$, whereas Dammers et al. [11] found a wall thickness variation in the brachial artery of approximately 15\%. All reported population spreads are thus significantly smaller than the measurement uncertainty that was used earlier. Therefore, we decided to use a maximum variation of $40 \%$ also in this study for the arteries as well as for the veins

The above described, holds for wall thickness tapering and thus $40 \%$ is used as maximum variation for this parameter.

Arterial and venous Young's modulus. The Young's moduli in the subclavian, axillary and brachial artery (\#36), the radial artery (\#37), the ulnar artery (\#38), the interosseus artery (\#39) and the aorta (\#40) could be fixed within their measurement uncertainty [121]. Also, the Young's moduli of the cephalic, axillary and subclavian vein (\#42), and of the basilic vein (\#41) could be fixed within their measurement uncertainty. The Young's modulus is inversely proportional to vessel distensibility. Distensibility measurements on brachial arteries of eleven ESRD patients of our hospital showed a variation of approximately $40 \%$ (unpublished data). We assumed that the variation in distensibility measurements similar to the variation in arterial Young's modulus and, in this study, we have thus extended the maximum variation to $40 \%$ for the Young's modulus of the arterial edges named above.

Preliminary venous distensibility measurements on eighteen ESRD patients showed an intersubject variation of approximately $20 \%$ [97]. In this study, this variation is used as population spread for the venous Young's modulus.

Mean brachial pressure. For the mean brachial pressure a population spread of approximately $15 \%$ was observed in twenty-six ESRD patients of our hospital (unpublished data) which is applied as maximum variation in this study.

Windkessel parameters. All parameters of the windkessels that close the right carotid artery (\#43, \#51 and \#59), the left subclavian artery (\#44, \#52 and \#60), the interosseus 
artery (\#47, \#55 and \#63), the vertebral artery (\#48, \#56 and \#64), the left carotid artery (\#50, \#58 and \#66) and the distal ulnar artery (\#46, \#54, \#62) were identified as parameters that could be fixed within their measurement uncertainty range [121].

The characteristic impedance is inversely proportional with the arterial cross-sectional area $[57,101]$. In the OpenClinica data, a diameter variation of approximately $20 \%$ in the subclavian artery is found. The same variation is used for the carotid arteries and the vertebral artery. The maximum variation of the characteristic impedances is then estimated to be $40 \%$. For the distal ulnar artery, a diameter variation of $30 \%$ was observed in the OpenClinica data resulting in a maximum variation for the characteristic impedance of $60 \%$. The same variation is used for the interosseus artery.

The windkessel resistance is determined by dividing the mean pressure by mean flow. The flow to the head is largely dependent on the metabolic needs of the brain [1]. It is therefore difficult to estimate the flow variation to the head. Dammers et al. [124] observed $30 \%$ variation in the mean velocity in the common carotid artery for ten healthy volunteers. Variations in the common carotid flow will be even larger because to obtain flow the velocity needs to be multiplied with cross-sectional area. Doppler ultrasound data for the subclavial flow in OpenClinica showed a population spread of approximately $50 \%$. We decided to use $50 \%$ as maximum variation in windkessel resistance, thus neglecting population spread in mean pressure for this parameter, as this would yield non-physiological inputs.

The maximum variation of the windkessel compliances are chosen to be $80 \%$ based on the $30 \%$ variation in the time constants observed by Leguy et al. [72] and the 50\% population spread in windkessel resistances, as the compliance is defined by the ratio between time constant and the windkessel resistance.

Anastomosis angle. The anastomosis angle was identified as a parameter that could be fixed, when it was varied from 15 to 90 degrees. Because anastomosis angles outside this range are clinically infeasible, this range is kept as it was.

\section{Analysis}

In this study, we present a methodology, that uses a global sensitivity analysis to identify model parameters, that can be fixed onto a generic value. The methodology is demonstrated using six different patient-specific datasets obtained from [101]. By using 6 datasets, the effect of different initial values is examined. For each patient two different configurations are simulated (lower and upper arm AVF) and color maps of the total sensitivity indices are produced. Model parameters with a total sensitivity index smaller 
than 0.05 for all patients and configurations are considered to be non-influential and are therefore identified as fixable parameters.

After identifying the fixable model parameters, it is examined if all the uncertainty domains for the six patients overlap. This overlapping region defines the values that can be used as generic value for that parameter. 


\subsection{Results}
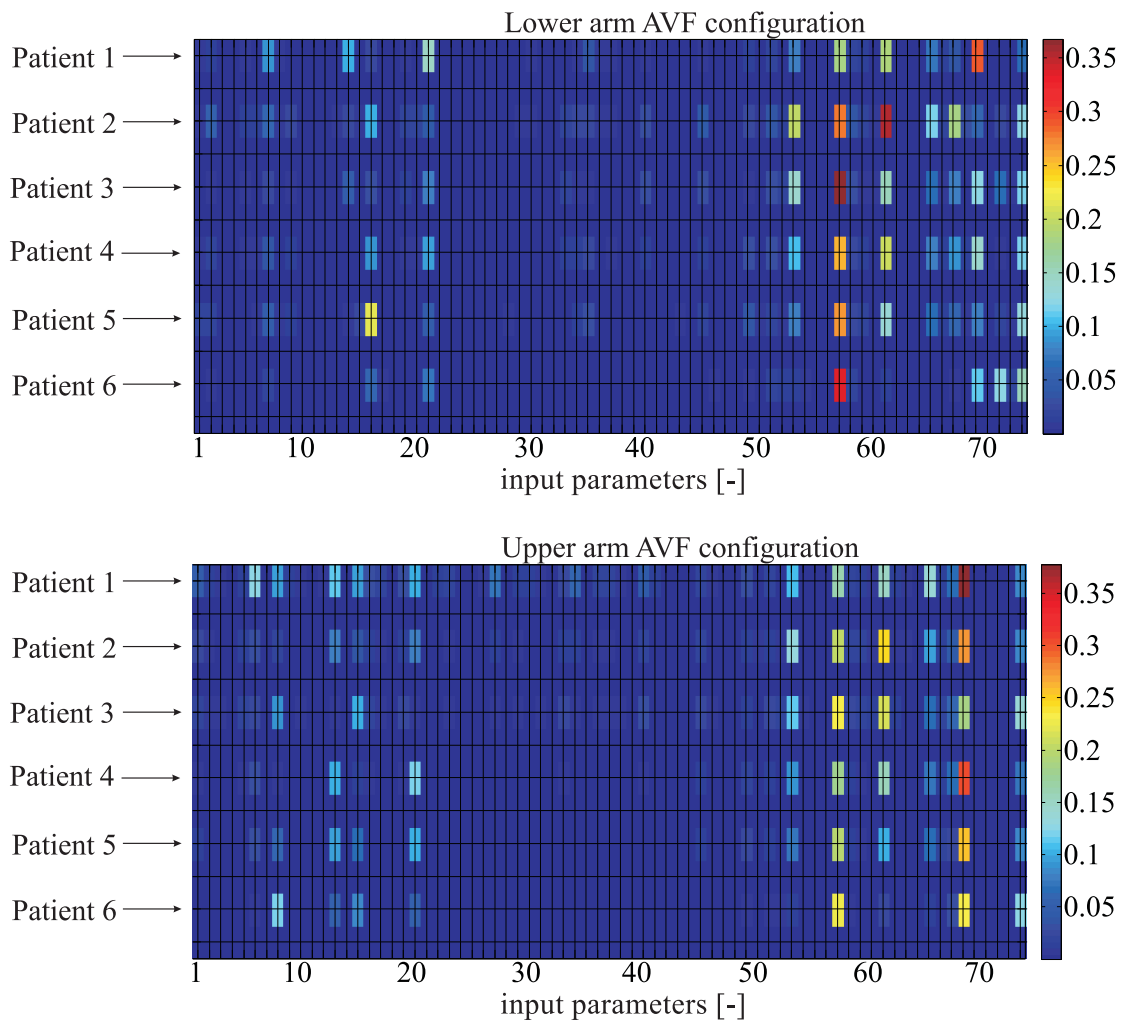

Figure 5.2: The total sensitivity indices for all input parameters with respect to the mean brachial flow in the lower arm AVF and upper arm AVF configuration.

The total sensitivity indices are shown in color maps for six patients and two AVF configurations (upper and lower arm AVF) of all input parameters with respect to the mean brachial flow and the systolic pressure in the distal radial artery (Figure 5.2 and 5.3 respectively). The input parameters that can be fixed for all patients and AVF configurations are summarized in Table 5.2, whereas the details are described below. Thereafter, the overlapping regions of the uncertainty domains are presented to define the generic values for the fixable model parameters. 


\section{Mean brachial flow}

The total sensitivity indices in Figure 5.2 (top) indicate that in case of the lower arm AVF configuration, the mean brachial flow is not significantly effected by the lengths of the subclavian, axillary and brachial artery (\#1), and of the ulnar artery (\#3), the interosseus artery (\#4) and the aorta (\#5). In addition, the length (\#6) of the median cubital and basilic vein are non-influential parameters, whereas the length of the radial artery (\#2) and cephalic, axillary and subclavian vein (\#7) have significant residual variances (0-9\%) in at most two patients. The vessel diameters can be fixed for all vessels except the radial artery (\#16, 0-22\%) and the cephalic, axillary and subclavian vein (\#21, 0-15\%) that show significant residual variances in at least four patients. The diameter tapering of the cephalic, axillary and subclavian vein (\#14) has a significant residual variance of $10 \%$ in patient 1 , while in all other patients this parameter can be fixed. For all other vessels, diameter tapering can be fixed. The wall thicknesses, changes in wall thickness, the Young's moduli, the mean brachial pressure (\#72) and the windkessel characteristic impedances of all edges can be fixed for all patients. The radial artery and aortic windkessel resistances (respectively \#53, residual variance from 0 to $20 \%$ and \#57, 18-37\%) and windkessel compliances (respectively \#61, 0-35\% and \#65, 0-12\%) cannot be fixed in at least five patients, whereas all other windkessel resistances and compliances can be fixed. In addition, the anastomosis angle (\#70) has total sensitivity indices smaller than 0.05 and can thus be fixed. The distributed flow (\#71, 0-12\%) can be fixed in four patients, whereas the intravenous pressure (\#67, 0-18\%) can be fixed in only two patients.

In case of the upper arm configuration, the arterial and venous vessel lengths can be fixed, except for the length of the subclavian, axillary and brachial artery (\#1,0-5\%), and the basilic, axillary and subclavian vein (\#6, 0-13\%) which are not fixable in one patient (Figure 5.2 (down)). Also the vessel diameters and vessel diameter tapering can be fixed for all vessels except for the subclavian, axillary and brachial artery (respectively \#15, 0$10 \%, \# 8,0-12 \%$ ), and the basilic, axillary and subclavian vein (respectively \#20, 0-12\% and \#13,0-11\%) that are not fixable in at least four patients. The wall thickness (\#34, $0-6 \%)$ and the tapering in wall thickness (\#27, 0-8\%) of the basilic, axillary and subclavian vein cannot be fixed in one patient, whereas for all other vessels the wall thickness and wall thickness tapering can be fixed. The Young's moduli, the mean brachial pressure (\#72) and the windkessel characteristic impedances can be fixed for all patients. Three other parameters can be fixed for only one patient. These are the radial artery windkessel resistance (\#53, 0-13\%), the radial artery and aortic windkessel compliances (respectively $\# 61,0-24 \%$ and \#65, 0-14\%). The aortic windkessel resistance cannot be fixed for all pa- 
tients (\#57, 16-23\%). All other windkessel parameters can be fixed for all patients. The anastomosis angle (\#70) and the distributed flow (\#71) of all vessels can be fixed for all patients, while the intravenous pressure $(\# 67,0-7 \%)$ can be fixed in halve of the patients.

\section{Distal radial artery systolic pressure}

In case of the lower arm AVF configuration, it can be observed in Figure 5.3 (top) that the radial artery length $(\# 2,0-8 \%)$ can be fixed in four out of six patients, whereas all other vessels lengths can be fixed for all patients. The diameters of all vessels can be fixed for all patients except for the subclavian, axillary and brachial artery (\#15, 0-8\%), for the radial artery (\#16, 0-13\%) and for the cephalic, axillary and subclavian vein (\#21, 0-6\%) that cannot be fixed in at least one out of six patients. For all patients, the diameter tapering, wall thickness and wall thickness tapering of all vessels do not significantly influence the distal systolic pressure (total sensitivity indices smaller than 0.05). The Young's modulus of the subclavian, axillary and brachial artery (\#36, 0-5\%) cannot be fixed in one patient. However, the Young's moduli of all other vessels can be fixed for all patients. In addition, the mean brachial pressure (\#72) can be fixed for all patients. The aortic characteristic impedance (\#49, 0-17\%) cannot be fixed in four out of six patients, while all other characteristic impedances can be fixed. The radial artery windkessel resistance (\#53, 0-21\%) can be fixed in two out of six patients, whereas the aortic windkessel resistance (\#57, $7-32 \%$ ) is influential in all patients. All other windkessel resistances can be fixed for all patients. The radial artery windkessel compliance (\#61, 0-39\%) and the aortic windkessel compliance (\#65, 0-11\%) can be fixed in respectively only one and three out of six patients, while all other windkessel compliances can be fixed for all patients. Furthermore, the anastomosis angle (\#70), the distributed flow (\#71) and the eccentricity ratio of the median cubital and basilic vein (\#68) can be fixed for all patients. The eccentricity ratio of the cephalic, axillary and subclavian vein (\#69,0-8\%) can be fixed in only one out of six patients, whereas the intravenous pressure (\#67, 0-13\%) can be fixed in four out of six patients.

The total sensitivity indices in Figure 5.3 (down), that are calculated for the distal systolic pressure, indicate that in case of the upper arm AVF configuration the vessel lengths can be fixed for all patients except for the lengths of the subclavian, axillary and brachial artery (\#1), and the basilic, axillary and subclavian vein (\#6) that have total sensitivity indices larger than 0.05 for patient 1 (respectively 6 and 15\%). In addition, the diameter (\#20) and diameter tapering (\#13), and the wall thickness (\#34) and the wall thickness tapering (\#27) of the basilic, axillary and subclavian vein can be fixed for all patients except one, for which they have a significant residual variance of respectively $9 \%, 9 \%, 6 \%$ and 

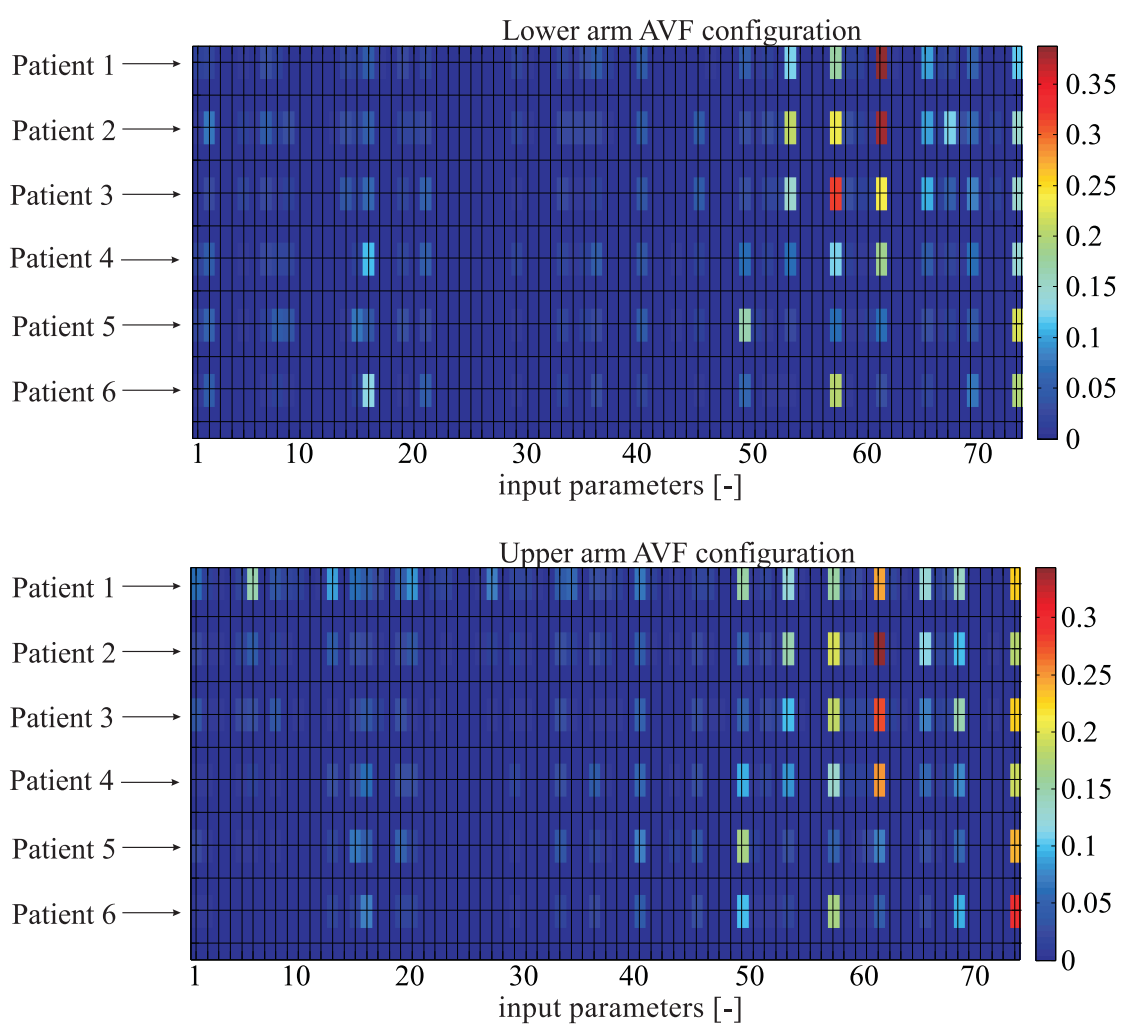

Figure 5.3: The total sensitivity indices for all input parameters with respect to the systolic pressure in the distal radial artery for the lower arm AVF and upper arm AVF configuration.

$7 \%$. For all other vessels, the diameter tapering, the wall thickness and the wall thickness tapering can be fixed. The diameters of the subclavian, axillary and brachial artery (\#15, $0-7 \%)$, of the radial artery (\#16,0-7\%) and of the aorta (\#19,0-5\%) can be fixed in respectively four, four and five out of six patients. All other vessel diameters can be fixed for all patients. The Young's modulus of the aorta (\#40, 0-7\%) can be fixed in only two out of six patients, whereas the Young's moduli of all other vessels can be fixed for all patients. Furthermore, it can be observed in Figure 5.3 (down) that the mean brachial pressure (\#72) and all characteristic impedances, except the aortic characteristic impedance (\#49, 0-17\%), can be fixed for all patients. The radial artery windkessel resistance (\#53, $0-15 \%$ ) can be fixed for only two out of six patients, whereas the aortic windkessel resis- 
tance (\#57, 5-19\%) cannot be fixed for any patient. All other windkessel resistances can be fixed. In addition, all windkessel compliances can be fixed except for the windkessel compliances of the radial artery (\#61, 0-34\%) and of the aorta (\#65, 0-13\%) that can only be fixed in respectively one and two out of six patients. The anastomosis angle (\#70), the distributed flow (\#71) and the intravenous pressure (\#67) can be fixed for all patients.

\section{Definition of population averaged constants}

In summary, for the two outputs and all patients and configurations 47 parameters were identified as fixable. The overlap of the uncertainty domains of the six patients for a fixable model parameter determines the values on which the model parameter can be fixed. In this way, for 37 ( 11 with full and 26 with partially overlap) out of 47 parameters a range of values could be found which are given in Table 5.2. For 10 out of 47 model parameters the uncertainty domains had no overlap. Typical examples of these three categories are shown in Figure 5.4. 
Table 5.2: The input parameters and their patient uncertainty domains $\Delta X_{i}$. Edges that are changed simultaneously are separated by commas. Note that for the upper arm AVF some venous edges are not in the model and therefore the related parameters are by definition non-influential in the upper arm AVF configuration. Model parameters that were selected in our previous study [121] as candidates for parameter fixing are shown in italics, whereas for the model parameters that are identified as fixable for all patients and both AVF configurations in the present study the range of generic values is given in the fourth column. If no range could be defined this is indicated by $\mathrm{X}$.

\begin{tabular}{|c|c|c|c|c|c|c|c|}
\hline 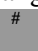 & $\begin{array}{l}\text { Parameters } \\
\text { Arterial lengths }\end{array}$ & $\Delta X_{i}$ & Fixable ranges & \# & $\begin{array}{l}\text { Parameters } \\
\text { Arterial Young's moduli }\end{array}$ & $\Delta X_{i}$ & Fixable ranges \\
\hline 2 & $\begin{array}{l}\text { innominate, subclavian, axillary and brachial } \\
\text { radial }\end{array}$ & $20 \%$ & & $\begin{array}{l}36 \\
37\end{array}$ & $\begin{array}{l}\text { innominate, subclavian, axillary, brachial } \\
\text { radial }\end{array}$ & $40 \%$ & \\
\hline 3 & ulnar proximal and distal & $20 \%$ & $5.4-8.0,14-21[\mathrm{~cm}]$ & 38 & ulnar proximal and distal & $40 \%$ & $2.2-2.5,2.2-2.25[\mathrm{MPa}]$ \\
\hline${ }_{5}^{4}$ & 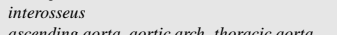 & $20 \%$ & 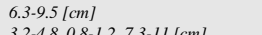 & 39 & interosseus & $40 \%$ & \\
\hline & $\begin{array}{l}\text { ascending aorta, aortic arch, thoracic aorta } \\
\text { Venous legnths }\end{array}$ & & $3.2-4.8,0.8-1.2,7.3-11[\mathrm{~cm}]$ & & $\begin{array}{l}\text { ascending aorta, aortic arch, thoracicic aorta } \\
\text { Venous Young's moduli }\end{array}$ & & \\
\hline 6 & median cubital and basilic vein ${ }^{\mathrm{a}}$ & $10 \%$ & & 41 & & $20 \%$ & $0.096-0.140 .0096-0.14[\mathrm{MPa}]$ \\
\hline 7 & cephalic, axillary and subclavian ${ }^{b}$ & $20 \%$ & & 42 & cephalic, axillary, subclavian ${ }^{b}$ & $20 \%$ & $0.096-0.14,0.096-0.14,0.096-0.14[\mathrm{MPa}]$ \\
\hline & $\begin{array}{l}\text { Arterial diameter tapering } \\
\text { innominate subclavion axillarv and brachial }\end{array}$ & & & & Characteristic impedances & & \\
\hline 9 & $\begin{array}{l}\text { Innominate, subclavian, axillary and brachial } \\
\text { radial }\end{array}$ & $10 \%$ & $\mathrm{x}$ & 43 & right carotid antery & $40 \%$ & $2.6-3.9\left[10^{8} \mathrm{~Pa} \cdot \mathrm{s} / \mathrm{m}^{3}\right]$ \\
\hline 10 & ulnar proximal and distal & $30 \%$ & $-4.4-6.8,1.7-6.8\left[10^{-4}\right]$ & ${ }_{45}^{44}$ & left subclavian artery & $20 \%$ & $2.0-2.9\left[10^{8} \mathrm{~Pa} \cdot \mathrm{s} / \mathrm{m}^{3}\right]$ \\
\hline 11 & interosseus & $30 \%$ & $-3.4-3.4\left[10^{-4}\right]$ & 46 & $\begin{array}{l}\text { radala artery } \\
\text { distal unar artery }\end{array}$ & $60 \%$ & \\
\hline 12 & ascending aorta, aortic arch, thoracic aorta & $15 \%$ & $-2.5-2.9,-2.1-2.1,1-1.4-2.3\left[10^{-3}\right]$ & 47 & interosseus artery & $60 \%$ & $100-160\left[10^{8} \mathrm{~Pa} \cdot \mathrm{s} / \mathrm{m}^{3}\right]$ \\
\hline 13 & $\begin{array}{l}\text { Venous diameter tapering } \\
\text { median cubital and basilic }{ }^{2}\end{array}$ & $10 \%$ & & 48 & $\begin{array}{l}\text { vertebral artery } \\
\text { thercis }\end{array}$ & $40 \%$ & $17-25\left[10^{8} \mathrm{~Pa} \cdot \mathrm{s} / \mathrm{m}^{3}\right]$ \\
\hline 14 & cephalic, axillary and subclavian b & $10 \%$ & & ${ }_{50}^{49}$ & $\begin{array}{l}\text { thoracict aotala } \\
\text { left cartid artery }\end{array}$ & $40 \%$ & 2.6-3.9[10 $\left.\mathrm{Pa} \cdot \mathrm{s} / \mathrm{m}^{3}\right]$ \\
\hline & $\begin{array}{l}\text { Arterial diameters } \\
\text {. }\end{array}$ & & & & Windkessel resistances & & \\
\hline $\begin{array}{l}15 \\
16\end{array}$ & $\begin{array}{l}\text { innominate, subclavian, axillary and brachial } \\
\text { radial }\end{array}$ & $10 \%$ & & 51 & right carotid artery & $50 \%$ & $1.4-1.8\left[10^{9} \mathrm{~Pa} \cdot \mathrm{s} / \mathrm{m}^{3}\right]$ \\
\hline 17 & $\begin{array}{l}\text { radal } \\
\text { ulnar proximal and distal }\end{array}$ & $30 \%$ & $2.4-2.8 .1 .9-2.20[\mathrm{~mm}]$ & 52 & left subclavian artery & $50 \%$ & \\
\hline 18 & interosseus & $30 \%$ & $1.0-1.5[\mathrm{~mm}]$ & 54 & $\begin{array}{l}\text { radiala artery } \\
\text { distal unartery }\end{array}$ & $50 \%$ & $x$ \\
\hline & ascending aorta, aortic arch, thoracic aorta & $10 \%$ & & 55 & interosseus artery & $50 \%$ & $\hat{x}$ \\
\hline 20 & $\begin{array}{l}\text { Venousus diametetrs } \\
\text { median cubital and basilic } \mathrm{a}^{\mathrm{a}}\end{array}$ & $10 \%$ & & 56 & vertebral artery & $50 \%$ & $21-29\left[10^{9} \mathrm{~Pa} \cdot \mathrm{s} / \mathrm{m}^{3}\right]$ \\
\hline 21 & cephalic, axillary and subclavian ${ }^{\mathrm{b}}$ & $10 \%$ & & $\begin{array}{l}57 \\
58\end{array}$ & $\begin{array}{l}\text { thoracic aorta } \\
\text { leff carotid artery }\end{array}$ & ${ }_{50 \%}^{25 \%}$ & 1.4-1.8[1109a $\left.\cdot \mathrm{s} / \mathrm{m}^{3}\right]$ \\
\hline 22 & $\begin{array}{l}\text { Change in arterial wall thickness } \\
\text { innominate, subclavian, axillary and brachial }\end{array}$ & $40 \%$ & $-6.4-6.4-3.1-3.4 .40 .8 .3 .6\left[10^{-4}\right]$ & & Windkessel compliances & & \\
\hline 23 & radial & $40 \%$ & 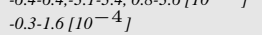 & 59 & 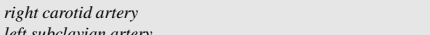 & $80 \%$ & $18-70\left[10^{-11} \mathrm{~Pa} / \mathrm{m}^{3}\right]$ \\
\hline 24 & ulnar proximal and distal & $40 \%$ & $-1.3-1.8,0.1-1.7\left[10^{-4}\right]$ & 61 & radial artery & $50 \%$ & \\
\hline 25 & interosseus & $40 \%$ & $-2.2-2.2 .2\left[10^{-4}\right]$ & 62 & distal unar artery & $80 \%$ & $x$ \\
\hline 26 & ascending aorta, aortic arch, thoracic aorta & $40 \%$ & $-13-13,-10-10,-9,0.9 .90\left[10^{-4}\right]$ & 63 & interosseus artery & $80 \%$ & $x$ \\
\hline 27 & $\begin{array}{l}\text { Change in venous wall thickness } \\
\text { median cubital, absilic }\end{array}$ & $40 \%$ & & $\begin{array}{l}64 \\
65\end{array}$ & $\begin{array}{l}\text { vertebral artery } \\
\text { thoracic aorta }\end{array}$ & $\begin{array}{l}80 \% \\
50 \%\end{array}$ & $1.2-4.7\left[10^{-11} \mathrm{~Pa} / \mathrm{m}^{3}\right]$ \\
\hline 28 & cephalic, axillary and subclavian $b$ & $40 \%$ & $X, 0.2-0.9,-2.8-0.3\left[10^{-4}\right]$ & 66 & left carotid artery & $80 \%$ & $18-70\left[10^{-11} \mathrm{~Pa} / \mathrm{m}^{3}\right]$ \\
\hline & Arterial wall thickness & & & & & & \\
\hline 29 & innominate, subclavian, axillary and brachial & $40 \%$ & 4.8-11, 3.6-5.7, 3.2-4.3 $\left[10^{-4} \mathrm{~m}\right]$ & $\begin{array}{l}67 \\
68\end{array}$ & $\begin{array}{l}\text { intravenous pressure } \\
\text { eccentricity ratio of median cubital and basilic vein a }\end{array}$ & & \\
\hline 30 & radial artery & $40 \%$ & $1.2-2.0\left[10^{-4} \mathrm{~m}\right]$ & & 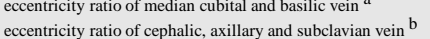 & $1.3-1.9^{\mathrm{c}}$ & \\
\hline 31 & ulnar proximal and distal & $40 \%$ & 2.1-3.0,1.7-2.2. $\left[110^{-4} \mathrm{~m}\right]$ & 70 & $\begin{array}{l}\text { eccentriticy yatio o c cephalic, axillary and subclavian vein } \\
\text { anasstomsis angle }\end{array}$ & $15-90^{c}$ & $15-90$ \\
\hline 32 & interosseus & $40 \%$ & $1.7-3.9 \cdot 9\left[10^{-4} \mathrm{~m}\right]$ & 71 & distributed flow & $40 \%$ & \\
\hline 20 & $\begin{array}{l}\text { ascending aorta, aortic arch, thoracic aorta } \\
\text { Venous wall thickness }\end{array}$ & $40 \%$ & 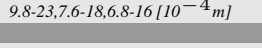 & $\begin{array}{l}72 \\
73\end{array}$ & $\begin{array}{l}\text { mean brachial pressure } \\
\text { mean aortic flow }\end{array}$ & 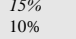 & \\
\hline $\begin{array}{l}34 \\
35\end{array}$ & $\begin{array}{l}\text { median cubital, basilic }{ }^{\mathrm{a}} \\
\text { cephalic, axillary and subclavian }^{b}\end{array}$ & $\begin{array}{l}40 \% \\
40 \%\end{array}$ & $1.4-1.5,1.6-2.0,2.7-3.7 .7\left[10^{-4} m\right]$ & & & & \\
\hline & er arm AVF the basilic, axillary and sub & vin & bined. & & & & \\
\hline
\end{tabular}




\subsection{Discussion}

Previously, we developed a distributed lumped parameter wave propagation model [101] that supports in decision-making for AVF surgery by predicting the change in mean brachial flow and distal systolic pressure after AVF creation. However, to apply this model in clinical practice, the model input parameters need to be adapted to patient-specific conditions and for this measurements on patients are required. Unfortunately, in clinical practice, not all parameters can be measured which results in sparse datasets. In addition, patient measurements are compromised by uncertainties. These sparse and uncertain input data will result in model output uncertainty. Therefore in a previous study [121], a framework was developed that was used to analyze the propagation of measurement uncertainties to output uncertainty by means of a variance-based global sensitivity analysis that uses a Monte Carlo experiment. This analysis showed that only for 16 out of 73 model input parameters it would be rewarding to improve the measurements, whereas 51 out of 73 parameters could be fixed within their measurement uncertainty domain. However, these parameters still need to be acquired which hampers the clinical applicability of our wave propagation model. Therefore in this study, we aimed to present a methodology to assess the model parameters that can be fixed onto a generic value.

The methodology was demonstrated using six patient-specific datasets. For the 51 model parameters that were previously [121] identified as non-influential, the uncertainty domain were extended and based on a population spread. Results showed that 47 model parameters could be fixed within this extended uncertainty domain for all patients and configurations. Two of these model parameters were not yet identified in our previous sensitivity study [121]. These parameters were the radial artery diameter tapering (\#9) and the radial artery characteristic impedance (\#45). Previously, they had a total sensitivity index of 0.05 in two out of six patients. Small deviations are possible because the precision of the estimates is 0.05 and their sensitivity indices are exactly at the used threshold for identifying influential parameters. Moreover, five input parameters were identified in the present study as significant for the outputs in one or both AVF configurations (Table 5.2), whereas they were not identified previously [121]. These parameters are the length and the diameter tapering of the subclavian, axillary and brachial artery, the radial artery length, the length of the cephalic, axillary and subclavian, and the aortic Young's modulus. The first four parameters effect the mean brachial flow which can physically be explained because all these parameters effect the viscous blood flow resistance, whereas the aortic Young's modulus might affect wave propagation and therefore the systolic pressure in the radial artery. These parameters thus became influential now 


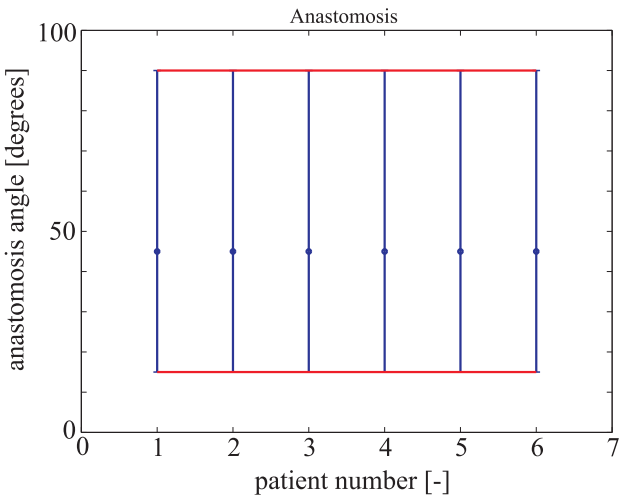

(a)

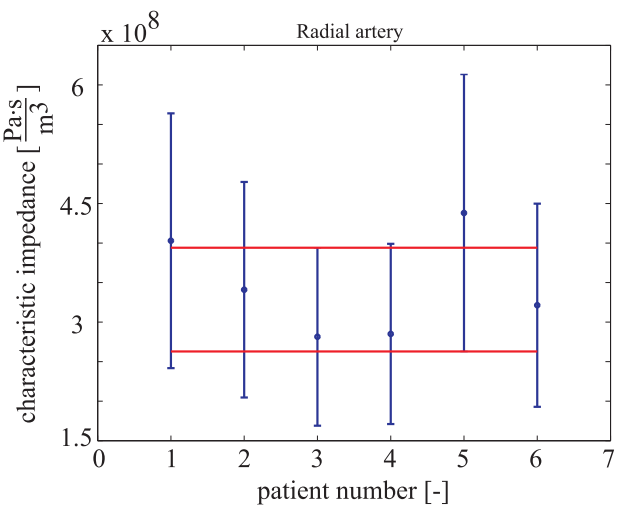

(b)

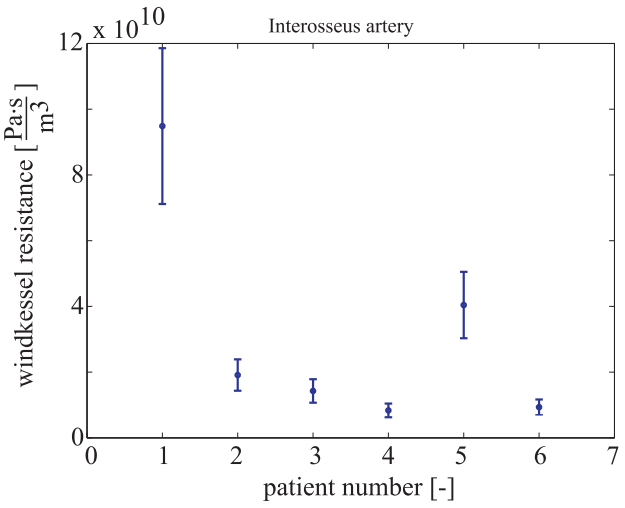

(c)

Figure 5.4: Three typical examples to present the intersection of patient uncertainty domains, indicated by the red lines and which determines the range of values in which the fixable model parameters can be fixed for all patients. In 5.4(a) the patient uncertainty domains of all six patients fully overlap while in 5.4(b) and 5.4(c) the patient uncertainty domains are partially and none overlapping respectively. 
their uncertainty domains are broadened. By considering the overlap of the uncertainty domains for all 47 fixable parameters, it was observed that in total 11 model parameters showed complete overlap between the uncertainty domains for all patients, while 10 model parameters showed no overlap at all. The remaining 26 model parameters had partially overlapping uncertainty domains for which the size of the intersection depends on the size of the uncertainty domain relative to the size of the population spread. The fact that the uncertainty domains for fixable input parameters had no overlap, results from the fact that the applied uncertainty domains were much smaller than the population spread in the six patient datasets. However, to avoid non-physiological combinations of input parameters, we decided to not further extend the uncertainty domains for these parameters. As a result, it is still necessary to measure these parameters. Completely overlapping domains result for model parameters that were generically, based on literature. Thus, as long as the uncertainty domains are close to the population spread, our methodology can be used to determine generic values for the fixable model parameters. However, the methodology was here only demonstrated on six patient datasets. Thus to set parameters, more patients should be included to get better estimates for the population spread and the uncertainty domains. Because it is difficult to define how many patients are needed for sufficient power, the methodology presented in this study can be seen as a self-learning approach in which each included patient increases the power of the sensitivity analysis. For future application, we suggest to iteratively redo the sensitivity analysis after inclusion of new patients, especially when for the patient input parameters are found that lay outside the current population spreads.

Although the results of the present study cannot yet be used to fix model parameters into generic values, the insight obtained can already be used when personalizing the wave propagation model. As our previous study [121] gives a direction for measurement improvements, this study indicates which parameters can be fixed. We propose to estimate these fixable model parameters based on literature and reduce the number of measurements and thus the burden on the patient. The assumptions made in this personalization step should thereafter be analyzed by a second sensitivity analysis in which the uncertainty in the assumptions is taken into account.

Note that the sensitivity analysis in this study assumes that all input parameters are independent. The methodology presented in this study, can be used for correlated input parameters if only a subset of the parameters in the total input space are correlated and the subsets are independent $[68,69,114]$. However, the possibility to distinguish between correlated parameters within a subset is lost and therefore this method fails if all parameters or most of them are correlated. Other quantitative methods to deal with correlated 
input parameters are the method of Ratto et al. [115], in which a biased estimator of the sensitivity index is derived, based on conditional density functions, or methods that are based on metamodels $[69,116,117,118]$, which are approximations of the actual model and derived by defining a simplified relation between the input parameters and the output of interest. The biggest advantage of metamodeling is that it is less computationally demanding than the actual model but designing a metamodel is rather complex and a sophisticated design of the computational experiment is needed, especially if you want to incorporate higher order interactions ( $>$ third order) [119]. These methods are further complicated by a large number of input parameters and by the large amount of data needed to determine the correlations between parameters. Therefore, we decided to use a Monte Carlo approach in this study. In addition, the computing resources (www.biggrid.nl) and the fast calculation time of the pulse wave propagation model allow us to run multiple simulations within reasonable time.

\subsection{Conclusion}

In this manuscript, we presented a self-learning approach that was able to identify 47 out of 73 model input parameters that could be fixed within their uncertainty domain. Furthermore, we found for 37 of these 47 model parameters generic values onto which the model parameters could be fixed. For the other 10 model parameters no generic value could be defined because the applied uncertainty domain was smaller than observed population spread in the six patient datasets. Therefore, it can be concluded that our proposed methodology can be used to determine generic values for the fixable model parameters as long as the applied uncertainty domains are close to to the population spread. However, the methodology was here only demonstrated on six patient datasets and therefore, to set parameters, more patients should be included to get better estimates for the population spread and the uncertainty domains. 


\section{Chapter 6}

\section{Model corroboration: An experimental study}

This Chapter is based on:

W. Huberts, K. Van Canneyt, S. Eloot, P. Segers, P. Verdonck, J.H.M. Tordoir, F.N. van de Vosse, E.M.H. Bosboom, Experimental validation of a pulse wave propagation model to predict vascular access surgery, Submitted, (2011) 


\begin{abstract}
End-stage renal disease (ESRD) patients treated with hemodialysis therapy, require a vascular access. Preferably, a vascular access is created in the arm, by surgically connecting an artery and vein, i.e. an arteriovenous fistula (AVF). The site for AVF creation is chosen by the surgeon based on clinical experience and preoperative vessel assessment. Still, AVFs are compromised by too low flows in lower arm AVFs or too large flows in upper arm AVFs. Previously, it was shown that a computational 1D-model is able to describe pressure and flow after AVF surgery. However, predicted flows significantly differed from measurements in $4 / 10$ patients. Differences can be attributed to inaccuracies in Doppler measurements and input data, to neglecting physiological mechanisms or to an incomplete physical description of the pulse wave propagation after AVF surgery. The physical description can be checked by corroborating against an experimental setup consisting of silicone tubes mimicking the aorta and arm vasculature both before and after AVF surgery, which is the aim of this study. In such an analysis, the output uncertainty resulting from measurement uncertainty in model input should be quantified. The computational model was fed by geometrical and mechanical properties collected from the setup. Pressure and flow waveforms were simulated and compared with experimental waveforms. The precision of the simulations was determined by performing a Monte Carlo study. It was concluded that the computational model was able to simulate mean pressures and flows accurately, whereas simulated waveforms were less attenuated than experimental ones, likely resulting from neglecting viscoelasticity. Furthermore, it was found that in the analysis output uncertainties, resulting from input uncertainties, can not be neglected and should thus be considered.
\end{abstract}




\subsection{Introduction}

Hemodialysis is a common treatment for end-stage renal disease (ESRD) patients [3, 4]. To facilitate hemodialysis, a well-functioning vascular access is needed. The vascular access should be able to provide a high blood flow ( $\geq 600 \mathrm{ml} / \mathrm{min}$ ) and should be easily accessible for repeated cannulation over time [6,7]. The preferred vascular access is an arteriovenous fistula (AVF) in the arm, which is a surgically created connection between an artery and vein (anastomosis), resulting in a significant flow increase (five- to thirtyfold) and vessel remodeling [6, 7, 8, 9, 10].

The AVF can be created both at wrist level (lower arm AVF) or at elbow level (upper arm AVF) $[11,12,13,14]$. In current clinical practice, the selection is based on the patient's medical history and the caliber of the involved vessels, i.e. when the caliber of the radial artery and the cephalic vein exceed $2 \mathrm{~mm}$ a lower arm AVF is created, otherwise an upper arm AVF. Despite the preoperative diagnostics, complications after AVF creation occur frequently. Lower arm AVFs are hampered by non-maturation in up to $50 \%$ of all cases which implies that six weeks after surgery, either the AVF is not dilated sufficiently (i.e. venous diameter $<6 \mathrm{~mm}$ ), the blood flow is too low $(<600 \mathrm{ml} / \mathrm{min})$, and/or the AVF is located too deep (i.e. venous depth $>6 \mathrm{~mm}$ ) $[6,7,8,9,10,12]$. Upper arm AVFs are hampered by long-term complications like distal ischemia and/or cardiac failure in $20 \%$ of all cases $[6,7,8,12]$. To reduce the number of complications and optimize surgical decision-making, tools to optimize the location for an individual patient are of interest. Because a too low brachial flow directly after surgery is associated with non-maturation $[10,32]$ and a postoperative flow larger than $30 \%$ of the cardiac output is associated with distal ischemia and cardiac failure $[11,12,13,14]$, the tools should aim at providing, preoperatively, a quantitative patient-specific estimate for the immediate postoperative flow. Previously, a pulse wave propagation model was developed that is able to simulate pressure and flow waveforms after upper and lower arm AVF surgery [101]. This computational model that is fed by patient-specific data was used to predict immediate postoperative flow in ten ESRD patients. It was shown that the model selected the same AVF location as an experienced surgeon in nine out of ten patients. Differences between model predictions and measurements might have several reasons. First, the accuracy of the measured flows, input data and boundary conditions might limit the correspondence between the simulated and measured flows. Second, the model neglects vascular adaptation and autoregulation of the peripheral bed which might result in differences between simulated and measured flows. A third reason might be that the physical description of the pulse wave propagation of the pressures and flows is incomplete. The way to determine if the 
physical description is complete, is by corroborating the pulse wave propagation model with an experimental setup which mimics the surgical AVF procedure and gives the possibility to determine a larger number and more accurate mechanical, geometrical and hemodynamic (pressure and flow waveforms) data than would be possible in an in-vivo situation $[125,126]$. In addition, vascular adaptation and regulation of the peripheral beds are excluded and do not influence the comparison of experimental and simulation results. Besides, an experimental setup can be used to validate the waveforms of the simulated pressures and flows which are needed when adaptation laws will be included in future work.

For arteries, in-vivo and experimental corroboration studies for pulse wave propagation models $[63,65,125,126,127]$ have been performed previously. However, these studies mainly focused on the systemic arterial tree with arterial flows much smaller than the flow after AVF creation. No corroboration study is reported that corroborates a pulse wave propagation model simulating the extreme flow increase after AVF surgery. Moreover, the propagation of measurement uncertainty in the model input to uncertainty in the model output (i.e. precision) was not analyzed previously. However, a quantitative estimate of the precision is required for a proper comparison between simulations and measurements. The output uncertainty can be assessed by using a method based on generalized chaos expansion ([71]) or by means of Monte Carlo simulations ([128]). The advantage of the method of Xiu et al. [71] is that less model runs are needed than for Monte Carlo simulations. However, the implementation is less straightforward as Monte Carlo simulations since the model equations are made stochastic and the most suitable polynomials for expansion of the stochastic function need to be selected. As a result, Monte Carlo simulations are more intuitive and therefore used in this study.

The aim of this study is thus to validate the previously developed distributed lumped parameter pulse wave propagation model for the prediction of pressure and flow before and after AVF creation. In this way it is determined if the physical description of the pulse wave propagation is correctly captured by the model. The uncertainty in pressure and flow waveforms, resulting from uncertainties in the model input parameters, is accounted for by using Monte Carlo simulations.

The manuscript is outlined as follows. First, the distributed lumped parameter pulse wave propagation model will briefly be described, followed by the experimental setup and how mechanical, geometrical and hemodynamic properties are determined. Next, how the model was adapted to the experimental conditions and how the model was corroborated with the experimental setup will be discussed. 


\subsection{Material and methods}

\subsubsection{The mathematical model}

The pulse wave propagation model used here was previously developed by Huberts et al. [101], and will only be described in short. The computational domain (Figure 6.1, bottom) includes the vessels of interest, in this case, the main vessels in the arm (pre- and postoperative). The vessels were divided in segments with a maximum length of $5 \mathrm{~cm}$. Each segment describes the local relation between pressure and flow. For the scope of modeling the effect of vascular access creation on blood pressure and flow distribution, segments are needed that represent arteries, veins and the anastomosis.

All arterial and venous segments were modeled with a lumped parameter model derived from local mass and momentum equations. The mass equation was simplified by assuming a compliance $C_{0}$ per unit length linearized around the mean pressure $p_{0}$, resulting in

$$
C_{0} \frac{\partial p}{\partial t}+\frac{\partial q}{\partial z}+\Psi=0
$$

in which $p$ is pressure, $q$ is flow and $\Psi$ the flow per unit length distributed to small side-branches that are not separately modeled by vascular segments. By assuming fullydeveloped incompressible Newtonian flow in a straight vessel and by using a time and frequency dependent approximated velocity profile to obtain an estimate for the wall shear stress, the momentum equation was reduced to:

$$
-\frac{\partial p}{\partial z}=R\left(\alpha_{0}\right) q+L\left(\alpha_{0}\right) \frac{\partial q}{\partial t} .
$$

Herein, $R\left(\alpha_{0}\right)$ and $L\left(\alpha_{0}\right)$ are respectively a resistor and an inductor depending on the Womersley number corresponding to the characteristic frequency of one heartbeat.

The anastomosis segment was modeled with a nonlinear resistance based on two semiempirical loss coefficients, $K_{v}$ and $K_{d}$, that describe the loss from the proximal artery to, respectively, the proximal vein and the distal artery and are defined by [91, 92]:

$$
\begin{gathered}
K_{v}=k_{1}\left(1-q^{*}\right)^{2}+q^{* 2}\left[k_{2} \cot \frac{\theta}{2}-k_{3}+\frac{k_{4}-k_{5} A^{*}}{A^{* 2}}\right]+k_{6} q^{*}\left(1-q^{*}\right)\left(1+\frac{1}{A^{*}}\right) \cot \frac{\theta}{2} \\
K_{d}=k_{7}\left(1-q^{*}\right)^{2}+k_{8} q^{* 2}-k_{9} q^{*}\left(1-q^{*}\right)
\end{gathered}
$$




\section{Experimental setup}

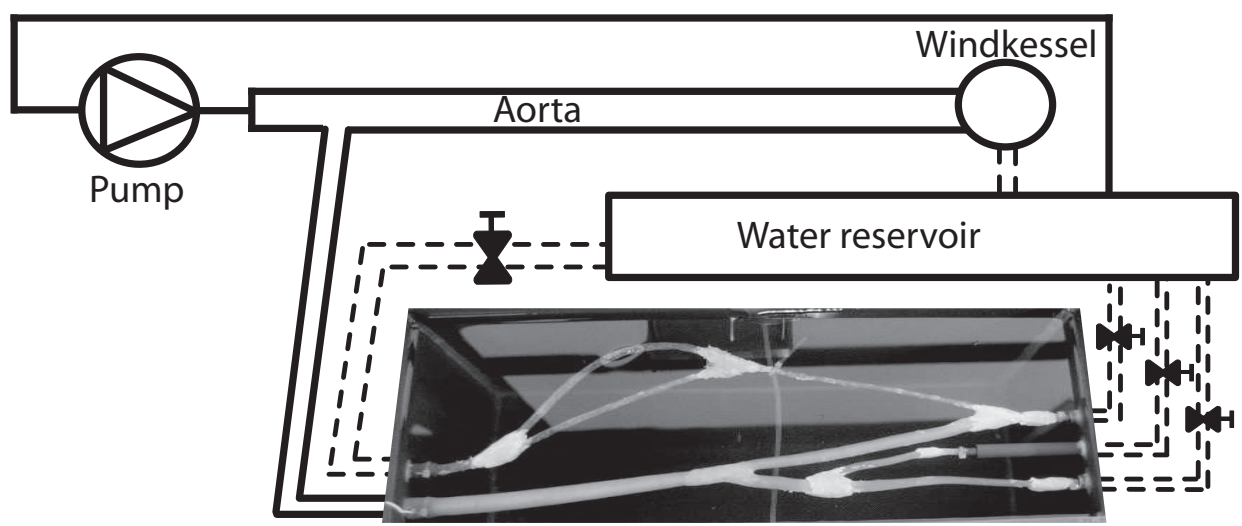

\section{Computational domain}

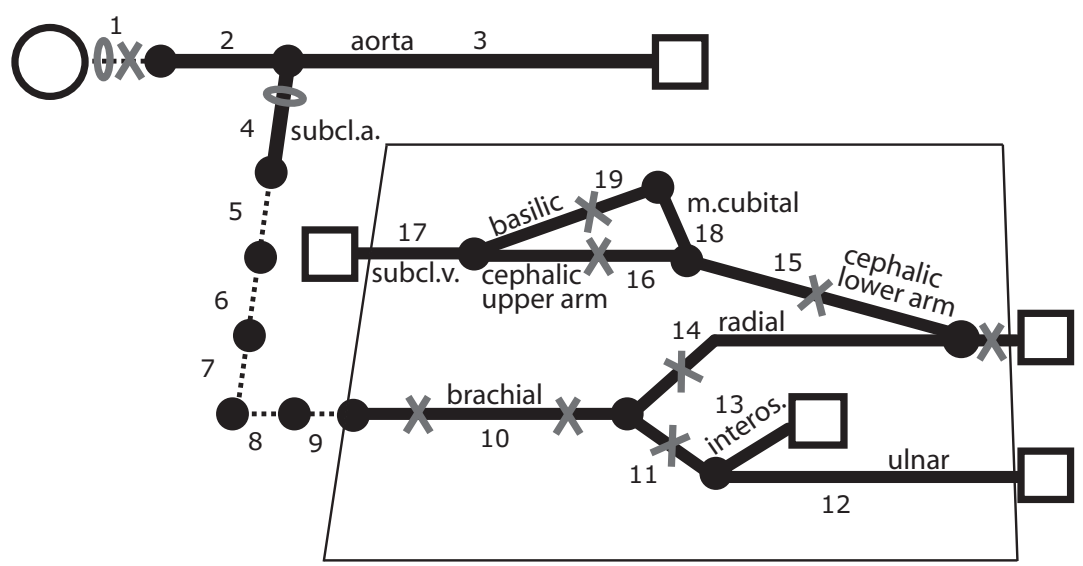

Figure 6.1: Network model mock loop scheme (top) and the computational domain (bottom). The numbers refer to the vessels given in Table 6.2. Pressure (x) and flow (0) waveform acquisition locations are shown. 
in which $q^{*}$ is the ratio between flow through the vein and flow through the proximal artery, $A^{*}$ is the ratio between cross-sectional area of the vein and cross-sectional area of the proximal artery and $\theta$ is the angle between proximal artery and proximal vein. Empirically determined values for the constants $k_{1}$ to $k_{9}$ are given in Table 6.1. The pressure drop from the proximal artery to the proximal vein, $p_{p}-p_{v}$, was now described by

$$
p_{p}-p_{v}=K_{v} \frac{1}{2} \rho \frac{q_{p}^{2}}{q_{v}^{2}} \frac{1}{A_{p}^{2}}\left|q_{v}\right| q_{v}=R_{v}\left(q^{*}, A^{*}, \theta, q_{v}, q_{p}, A_{p}\right) q_{v}
$$

whereas the pressure drop from the proximal artery to the distal artery, $p_{p}-p_{d}$, was expressed by

$$
p_{p}-p_{d}=K_{d} \frac{1}{2} \rho \frac{q_{p}^{2}}{q_{d}^{2}} \frac{1}{A_{p}^{2}}\left|q_{d}\right| q_{d}=R_{d}\left(q^{*}, A^{*}, \theta, q_{d}, q_{p}, A_{p}\right) q_{d}
$$

Herein, $A_{p}$ is the cross-sectional area of the proximal artery, $\rho$ the fluid density and $q_{p}, q_{v}$ and $q_{d}$ are respectively the flow in the proximal artery, proximal vein and the distal artery. Parts of the cardiovascular system, for which no detailed information on pressure and flow was required, were truncated and terminated with three-element windkessel models. Assembling all lumped segments resulted in a system of differential equations that describes the pulse wave propagation of the pressure and flow waveforms. This system was solved by numerical integration applying the trapezium rule for implicit time integration [101]. On the first node, a measured (aortic) flow was prescribed, whereas the venous outflow was closed with a fixed intravenous pressure.

The pressure and flow waveforms simulated by the pulse wave propagation model were corroborated with experimentally derived pressure and flow waveforms. The input parameters (e.g. $R\left(\alpha_{0}\right), L\left(\alpha_{0}\right)$ and $C_{0}$ ) of the pulse wave propagation model should therefore be adapted to the experimental setup. For this, geometrical (vessel length, vessel diameters) and mechanical characteristics of the vessels (vascular compliance) were mandatory. Furthermore, information on anastomosis configuration (location, angle), windkessel parameters, fluid properties (density and dynamic viscosity), intravenous pressure and an input (aortic) flow waveform were required. How these input parameters were obtained will be discussed in Section 6.2.3. 
Table 6.1: Semi-empirical determined dimensionless constants $k_{1}$ to $k_{9}$ for equation (6.3) and (6.4) $[91,92]$.

$\begin{array}{ccccccccc}k_{1} & k_{2} & k_{3} & k_{4} & k_{5} & k_{6} & k_{7} & k_{8} & k_{9} \\ 0.95 & 1.3 & 0.3 & 0.4 & 0.1 & 0.4 & 0.03 & 0.35 & 0.2\end{array}$

\subsubsection{The experimental model}

\section{In-vitro experimental setup}

The experimental silicone tube model, mimicking the anatomy and physiology of the human vasculature in the arm, was built based on patient-specific data obtained in a previous study [101]. The arterial geometrical data, obtained from magnetic resonance (MR) and ultrasound (US) examinations, were used to generate a three-dimensional (3D) CADmodel which was used as benchmark for manufacturing the 3D silicone tube model. For the construction of the silicone arterial model, Rapid Prototype models and dip-coating techniques were used (in-house techniques and knowledge). The venous silicone model was based on geometrical data obtained from US measurements of an ESRD patient and was constructed by painting silicone, layer by layer, on a steel rod [129]. This technique was chosen over dip-coating techniques as it was expected to result in lower wall thicknesses and thus lower stiffness. To mimic the AVF, the arterial and venous silicone models were connected by an anastomosis with an aperture of approximately $15 \mathrm{~mm}^{2}$ and an inplane angle of $30^{\circ}$ [28]. The resulting silicone network model consisted of the brachial, radial, ulnar and interosseus artery (arterial tract) and of the cephalic, basilic, median cubital, axillary and subclavian vein (venous tract).

This network model was built in a mock loop [76, 77] including a silicone aorta and subclavian artery constructed by Hemolab B.V.(Eindhoven, The Netherlands)(Figure 6.1, top). The arterial and venous silicone network was placed in a water tank to allow for ultrasound measurements. The complete circulation circuit was filled with water and the water was pumped through the mock loop by a step pump (Parker Hannifin GmbH; Offenburg, Germany) that was regulated by a normalized modified sinus-squared ISO5840 function (Figure 6.2) in LabView (National Instruments Inc., version 7.1, Austin, Texas, USA). This resulted in a pump flow rate of approximately $51 / \mathrm{min}$ which is comparable to a physiological cardiac output [1]. The fluid entered the proximal aorta via a flexible valve (Hemolab B.V) and could thereafter flow in two directions: to the distal aorta which was closed by a windkessel, and to the subclavian artery which was the inflow artery of the silicone network model. This silicone network model had three outflows in the arterial 
part (at radial, ulnar and interosseus artery) and one in the venous part (at the subclavian vein). For mimicking the preoperative situation, the venous outflow was closed with a clamp, whereas for the postoperative situation the clamp was released to mimic the presence of a lower arm AVF. The lower arm AVF was chosen because it involves the largest arterial and venous network and thus the most complex topology was studied.

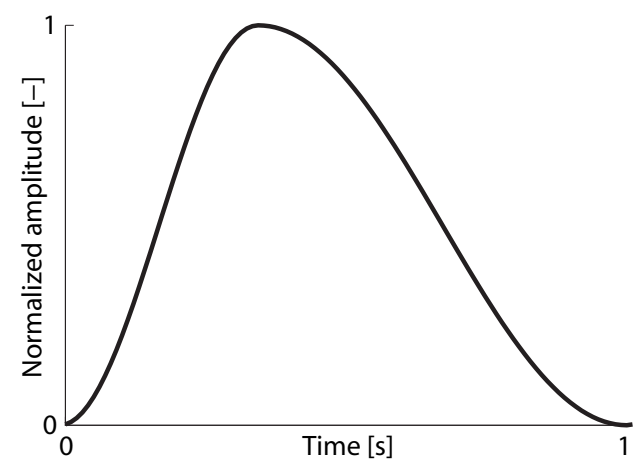

Figure 6.2: Pump input function (modified sinus-squared ISO5840)

The computational domain for the simulations consisted of a truncated part of the aorta, the subclavian, brachial, radial, ulnar and interosseus arteries, the anastomosis, the cephalic, median cubital, basilic, axillary and subclavian veins, and, in addition, all connecting tubes (Figure 6.1, bottom).

\section{Measurement techniques}

Geometrical measurements. The lengths of all arteries, connecting tubes and veins were measured using a ruler. In addition, the ruler was used to determine the location of the anastomosis with respect to the brachial bifurcation. The proximal and distal arterial and venous diameters were measured at working pressure with ultrasound (Picus ultrasound system, ESAOTE B.V., The Netherlands). The diameters of connecting tubes were determined with a micrometer.

Flow measurements. Flow waveforms were measured, at the proximal aorta and the proximal subclavian artery (Figure 6.1) with a Transonic flow meter acquisition system 
(Team 21 Compatible; Transonic Systems Inc., Ithaca, NY, USA). The signal was sampled at $500 \mathrm{~Hz}$ using a data-acquisition (DAQ) unit (BMC-2090, National Instruments Inc.). The acquisition software was programmed in LabView (National Instruments Inc., version 7.1, Austin, Texas, USA). For all outflow tracts, the time-averaged outflows were measured volumetrically.

Pressure measurements. Pressure waveforms were measured, using pressure transducers (P10EZ-1, Becton Dickinson Critical Care Systems; Singapore) connected to a multichannel compact amplifier system (PICAS; Peekel Instruments, Rotterdam, The Netherlands). The measurements were performed at predefined locations along the experimental model (Figure 6.1) by sliding a fluid-filled epidural catheter (Portex Epidural Catheter; Smiths Medical ASD, inc. Keene, USA) through the experimental model. The pressure transducers were also connected to the DAQ-unit.

Mechanical properties of the vessels. The diameter change (distension) of the arteries and veins was measured by using wall-tracking software (Artlab) that is incorporated in the Picus ultrasound system (ESAOTE B.V., The Netherlands). Since pressures were measured simultaneously, the distension, $\Delta d$, and the pulse pressure, $\Delta p$, were used to determine the local distensibility, $D$ :

$$
D \approx \frac{1}{A_{0}} \frac{\Delta A}{\Delta p}=\frac{2 d_{d} \Delta d+(\Delta d)^{2}}{d_{0}{ }^{2} \Delta p}
$$

in which $A_{0}$ and $d_{0}$ are the cross-sectional area and the diameter at mean pressure, and $d_{d}$ the diastolic diameter. The distensibility of the subclavian artery was estimated by using its wall-thickness-to-radius ratio of 0.1 and by assuming the Young's modulus equal to the aorta of the experimental setup, as the subclavian artery was made from the same material. The distensibilities of the connecting tubes were assessed from static volumetric compliance measurements or set to $10^{-7} \mathrm{~Pa}^{-1}$ otherwise.

Pressure drop over the anastomosis. The extra pressure drop over the anastomosis was captured via a minor loss coefficient, defined as $K_{\text {loss }}=\frac{\Delta p}{\frac{1}{2} \rho v^{2}}$, in which $\Delta p$ is the pressure drop from the inflow artery to the outflow vein, $\rho$ is the density, and $v$ is the mean fluid velocity in the inflow artery. $K_{\text {loss }}$ was determined by measuring the pressure drop as function of the Reynolds numbers from $\mathrm{Re}=200$ to 3500 (Figure 6.3A). 

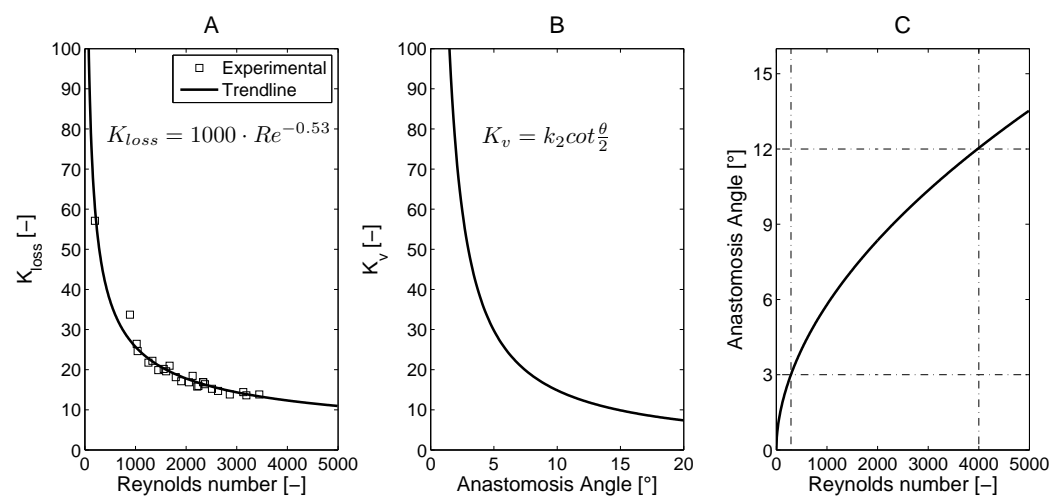

Figure 6.3: The experimentally derived minor loss coefficient as function of the Reynolds number (A) and the reduced minor loss equation (Equation (6.8)) implemented in the wave propagation model developed by Huberts et al. [101] as function of anastomosis angle (B). In panel C, the comparison between the experimentally derived loss coefficient (Reynolds number) and the loss coefficient implemented in the wave propagation model (Anastomosis angle) is shown.

\subsubsection{Experimental corroboration: experiment versus pulse wave prop- agation model}

\section{Model parameters for the pulse wave propagation model}

The input parameters for the pulse wave propagation model were derived from the measurements and are given in Table 6.2. In addition, uncertainty domains of these input parameters were estimated as they are input for the uncertainty analysis.

Geometrical parameters. The measured lengths were directly used in the pulse wave propagation model and the uncertainty domain was set to $\pm 5 \%$, which equals to a measurement error of $1 \mathrm{~mm}$ in the shortest vessel. Also the position of the anastomosis was varied within $\pm 5 \%$. The uncertainty domain for the diameters was set to $\pm 10 \%$. For the arterial and venous diameters, linear tapering within a vessel was assumed. The influence of tapering was investigated by changing the smallest diameter of a vessel while changing the largest diameter in the opposite direction. The uncertainty domain for these changes were based on $10 \%$ of the smallest diameter. 
Table 6.2: All input data for the wave propagation model that are derived from measurements on the experimental setup. The mean pressure, mean flow and time constant are only given for the vessels that are closed by a windkessel segment. In brackets the estimated measurement uncertainty is given in percentages. The vessels that are changed simultaneously during the uncertainty analysis are indicated by the same letters, i.e.: $c, d, e$ or $f$.

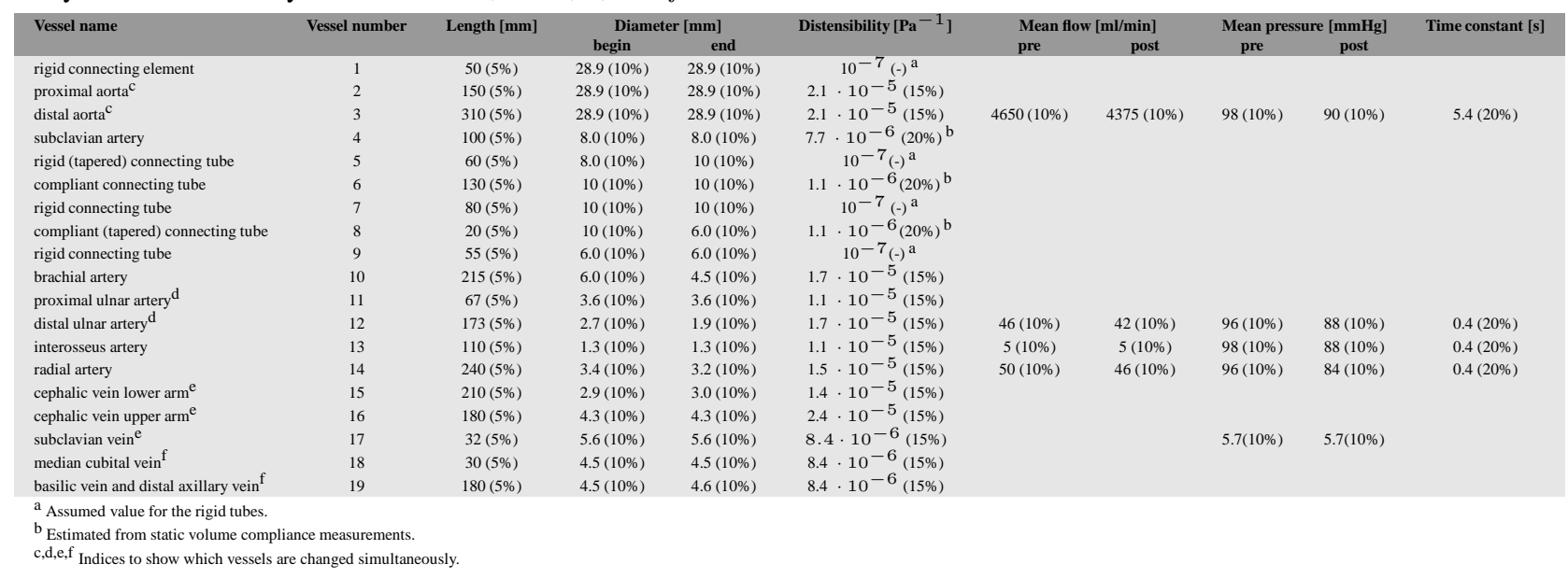


Mechanical parameters. For the pulse wave propagation model, the local vessel wall compliance per segment length was needed. This was derived by multiplying the distensibility in Table 6.2 by the local cross-sectional area, thus assuming a constant distensibility within each vessel. For the distensibilities obtained with ultrasound, the measurement uncertainty domain was $\pm 15 \%$, whereas for the distensibilities derived by volume compliance measurements, the measurement uncertainty domain was $\pm 20 \%$.

Pressure drop over the anastomosis. To match the minor loss coefficients in the pulse wave propagation model with the measured loss coefficient, we assumed that the majority of the proximal artery flow flows through the proximal vein $\left(q^{*} \approx 1\right)$ and that the arterial and venous cross-sectional areas were almost similar $\left(A^{*} \approx 1\right)$. The equations $(6.3)$ and (6.4) then reduce to

$$
K_{v}=k_{2} \cot \frac{\theta}{2}-k_{3}+k_{4}-k_{5}=k_{2} \cot \frac{\theta}{2} \quad \text { and } \quad K_{d}=k_{8} .
$$

This relationship for $K_{v}$ is shown in Figure 6.3B. For an anastomosis angle, $\theta$, between 3 and 12 degrees, the loss coefficient, $K_{v}$, matches the experimentally determined loss coefficient, $K_{\text {loss }}$, in the range of Reynolds numbers 250-4000 (Figure 6.3C). Finally, the loss coefficient, $K_{v}$, was modeled by equation (6.3), while the anastomosis angle was varied between 3 and 12 degrees. Because the flow through the distal artery was small compared to the flow through the proximal vein, loss coefficient, $K_{d}$, was neglected.

Constants. The fluid used in the experiment was water which has, at room temperature $\left(20^{\circ} \mathrm{C}\right)$, a density of $10^{3} \frac{\mathrm{kg}}{\mathrm{m}^{3}}$ and a dynamic viscosity of $10^{-3} \mathrm{~Pa} \cdot \mathrm{s}$. These model parameters would hardly vary during the experiment and therefore the uncertainty domains were set to $\pm 1 \%$ and $\pm 5 \%$, respectively.

Boundary conditions. The peripheral resistance, $R_{p}$, of each windkessel was determined as the ratio of local mean pressure and local mean flow. The local mean flow was assessed by the volumetric measurements, while the mean pressure was assessed by timeaveraging the measured local pressure waveforms. The characteristic impedance, $Z_{0}$, was assessed by using $\sqrt{\frac{\rho}{D A_{0}{ }^{2}}}$ [57] in which $\rho$ is the blood density, $D$ the local distensibility and $A_{0}$ the cross-sectional area. Finally, the compliance, $C_{v}$, for each windkessel was determined by dividing the time constant, $\tau$, by the difference between the peripheral resistance and the characteristic impedance $\left(R_{p}-Z_{0}\right)$. For the aorta, the time constant was assessed from the local pressure waveform via the "area method" [46]. The time constant 
in the radial artery was assessed from multiplying the local peripheral resistance with the compliance of the outflow tube. For the ulnar and interosseus arteries, the radial artery time constant was assumed. As a result, the time constant was $5.4 \mathrm{~s}$ in the aorta and 0.4 $\mathrm{s}$ in the other outflow arteries. In the uncertainty analysis, the time constants were varied by $\pm 20 \%$.

For the inflow boundary at the aorta (i.e. the ascending aorta flow), we used the pre- and postoperative aortic flow measurements, with an estimated uncertainty domain of $\pm 5 \%$. As outflow condition at the subclavian vein, we used the measured mean pressure with an uncertainty of domain of $\pm 10 \%$.

\section{Analysis}

Simulations with the pulse wave propagation model were performed for both the preand postoperative configuration. To examine the propagation of input uncertainty to the output, Monte Carlo simulations were performed. Varying all input parameters independently within their uncertainty domain might result in non-physiological combinations of input parameters and thus non-physiological output (e.g. a small artery continuing in a larger artery). Therefore, the parameters of adjacent vessels were changed simultaneously (Table 6.2). This resulted in 44 independent input parameters for the preoperative configuration and 58 parameters for the postoperative configuration. The input samples were generated by Latin Hypercube sampling to get a full coverage of the parameter input space [111]. The sampling and calculations were performed within a computational framework that was developed earlier in collaboration with Philips Research (Eindhoven, The Netherlands) [121].

For the preoperative configuration, the simulated pressure waveforms in the aorta, the proximal and distal brachial artery, the proximal and distal radial artery and the proximal ulnar artery were compared to the measured pressure waveforms (Figure 6.1, bottom). This was extended by the distal and proximal cephalic vein, and the basilic vein for the postoperative configuration. The flow waveforms were compared at proximal aorta and subclavian artery. For the comparisons, the median of the simulated pressure and flow waveforms of all Monte Carlo simulations was used as well as a confidence interval, formed by the $25^{\text {th }}$ and $75^{\text {th }}$ percentile interval. 


\subsection{Results}

\subsubsection{Preoperative results}

Table 6.3 shows that the simulated mean flows correspond to the measured mean flows in the aorta, subclavian artery, the radial artery and the ulnar artery. The shapes of the simulated aorta and subclavian artery flow waveforms correspond to the measured flow waveforms, though the simulated subclavian artery flow appears less attenuated (Figure 6.4).

For all arteries, simulated and measured mean pressures are similar (Table 6.3). The shapes of the simulated pressure waveforms (Figure 6.4) correspond to the measured pressure waveforms, although the simulated pressures are less attenuated resulting in a more pronounced second peak, which is clearer in distal pressure waveforms. For the aortic pressure waveform, a phase difference of approximately $50 \mathrm{~ms}$ is observed. Presumably, the timing of the measured aortic pressure waveform is inaccurate as the systolic pressure peak arrives before the flow peak.

\subsubsection{Postoperative results}

During the analysis of the postoperative results it was observed that, in first instance, the resistance in the distal cephalic vein was not properly determined. After excising the distal cephalic vein from the setup, a non-smooth vessel lumen that was twisted over the full length was found resulting in a large pressure drop of $24 \mathrm{mmHg}$ from the distal cephalic vein to the proximal cephalic vein (Table 6.4). This pressure drop was approximately ten times larger than the expected pressure drop as calculated from Poiseuille's law. As this additional pressure drop was not captured by the model, all Monte Carlo simulations were performed once more with a tenfold increased resistance in the distal cephalic vein with an uncertainty of $\pm 10 \%$. Since vessel abnormalities could also be present in human vasculature and the resulting additional pressure drop has significant effect, the results of both analyses, with and without the extra resistance, are presented in this section.

Without the improved resistance of the distal cephalic vein, the mean pressures are underestimated by the simulation, especially the mean pressure in the distal cephalic vein, which is underestimated by $23 \mathrm{mmHg}$. As in the preoperative simulations, the simulated arterial pressure waveforms (Figure 6.5) are less attenuated than the experimentally measured ones. In addition, a similar phase difference in the aorta is observed. The shape of the measured and simulated venous pressure waveforms (Figure 6.6) are similar, although the differences are larger than for the arterial site, i.e. a phase difference of approximately 
$50 \mathrm{~ms}$ in the proximal cephalic and basilic vein, and a pressure difference in the distal cephalic vein.

Postoperatively, the simulated mean flows in the aorta, distal radial artery and ulnar artery coincide with the measured mean flows (Table 6.4). The mean flows in the subclavian artery and the distal cephalic vein are overestimated by the simulations but are still within physiological ranges $[6,7,14]$.

When considering the additional resistance of the distal cephalic vein, the simulated mean flow of the subclavian artery and the distal cephalic vein in Table 6.4 now correspond to the measured mean flow. Note further that the shapes of the waveforms are not significantly affected by the adapted model (Figure 6.7 and 6.8). Besides a better agreement between measured and simulated flows, this is also the case for the pressures. In Table 6.4 , it can be observed that the previous observed underestimation of the measured mean arterial pressures are reduced, while measurements and simulations now coincide. This especially holds for the distal cephalic vein pressure. 
Table 6.3: Measured preoperative mean pressures and flows and their simulated values.

\begin{tabular}{|c|c|c|c|c|}
\hline & \multicolumn{2}{|c|}{ Mean pressure [mmHg] } & \multicolumn{2}{|c|}{ Mean flow $[\mathrm{m} / / \mathrm{min}]$} \\
\hline & measured $^{\mathrm{a}}$ & simulated $^{b}$ & measured $^{\mathrm{a}}$ & simulated $^{\mathrm{b}}$ \\
\hline proximal aorta & 95 & $93(88,99)$ & 4750 & $4747(4682,4813)$ \\
\hline subclavian artery & & & 101 & $98(36,161)$ \\
\hline proximal brachial artery & 98 & $93(88,99)$ & & \\
\hline distal brachial artery & 98 & $93(87,99)$ & & \\
\hline proximal ulnar artery & 98 & $93(87,99)$ & 46 & $44(35,53)$ \\
\hline proximal radial artery & 98 & $93(87,99)$ & 50 & $48(34,63)$ \\
\hline distal radial artery & 98 & $92(86,99)$ & & \\
\hline
\end{tabular}

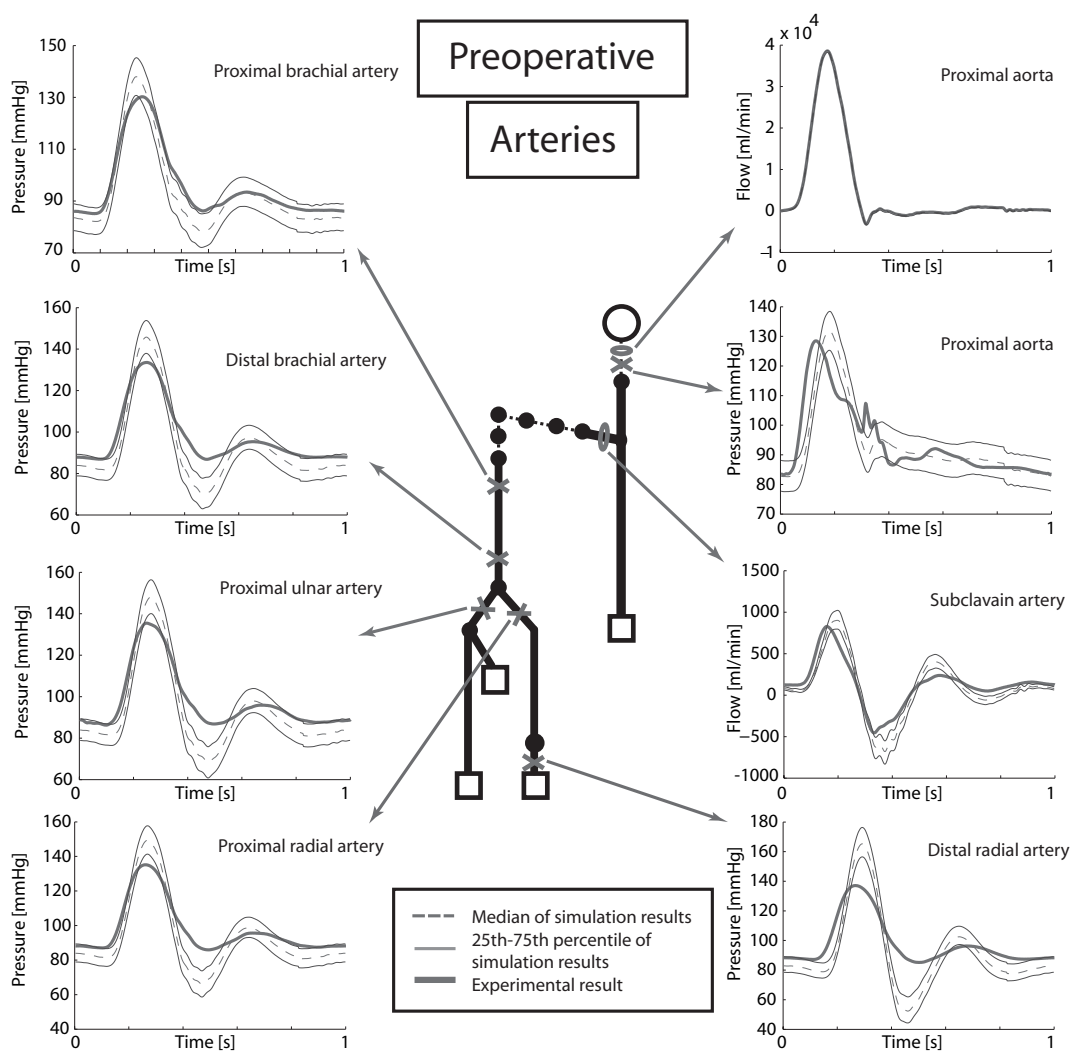

Figure 6.4: The preoperative pressure and flow waveforms on several arterial locations. 
Table 6.4: Measured postoperative mean pressures and flows and their simulated values.

\begin{tabular}{|c|c|c|c|c|c|c|}
\hline & \multicolumn{3}{|c|}{ Mean pressure $[\mathrm{mmHg}]$} & \multicolumn{3}{|c|}{ Mean flow $[\mathrm{ml} / \mathrm{min}]$} \\
\hline & measured $^{\mathrm{a}}$ & simulated $\mathbf{b}$ & $\begin{array}{l}\text { simulated } \\
\text { with extra } R^{b}\end{array}$ & measured $^{\mathrm{a}}$ & simulated $^{\mathbf{b}}$ & $\begin{array}{l}\text { simulated } \\
\text { with extra } R^{\mathrm{b}}\end{array}$ \\
\hline proximal aorta & 90 & $83(79,88)$ & $86(81,91)$ & 4750 & $4748(4676,4821)$ & $4740(4667,4812)$ \\
\hline subclavian artery & & & & 378 & $508(422,594)$ & \\
\hline proximal brachial artery & 90 & $83(78,87)$ & $85(80,91)$ & & & \\
\hline distal brachial artery & 90 & $81(76,87)$ & $85(79,90)$ & & & \\
\hline proximal ulnar artery & 88 & $81(76,87)$ & $84(79,90)$ & 42 & $38(31,46)$ & $40(32,47)$ \\
\hline proximal radial artery & 86 & $80(75,86)$ & $84(78,89)$ & - & & \\
\hline distal radial artery & 84 & $76(71,82)$ & $81(76,86)$ & 46 & $42(30,53)$ & $44(34,55)$ \\
\hline distal cephalic vein & 31 & $8.3(7.5,9.2)$ & $28(25,32)$ & $285^{\mathrm{c}}$ & $420(352,490)$ & $270(239,305)$ \\
\hline proximal cephalic vein & 7.0 & $6.0(5.8,6.2)$ & $5.9(5.8,6.0)$ & & & \\
\hline basilic vein & 6.0 & $6.0(5.8,6.2)$ & $5.9(5.8,6.0)$ & & & \\
\hline subclavian vein & $5.7^{\mathrm{d}}$ & $5.7^{\mathrm{d}}$ & $5.7^{\mathrm{d}}$ & & & \\
\hline
\end{tabular}

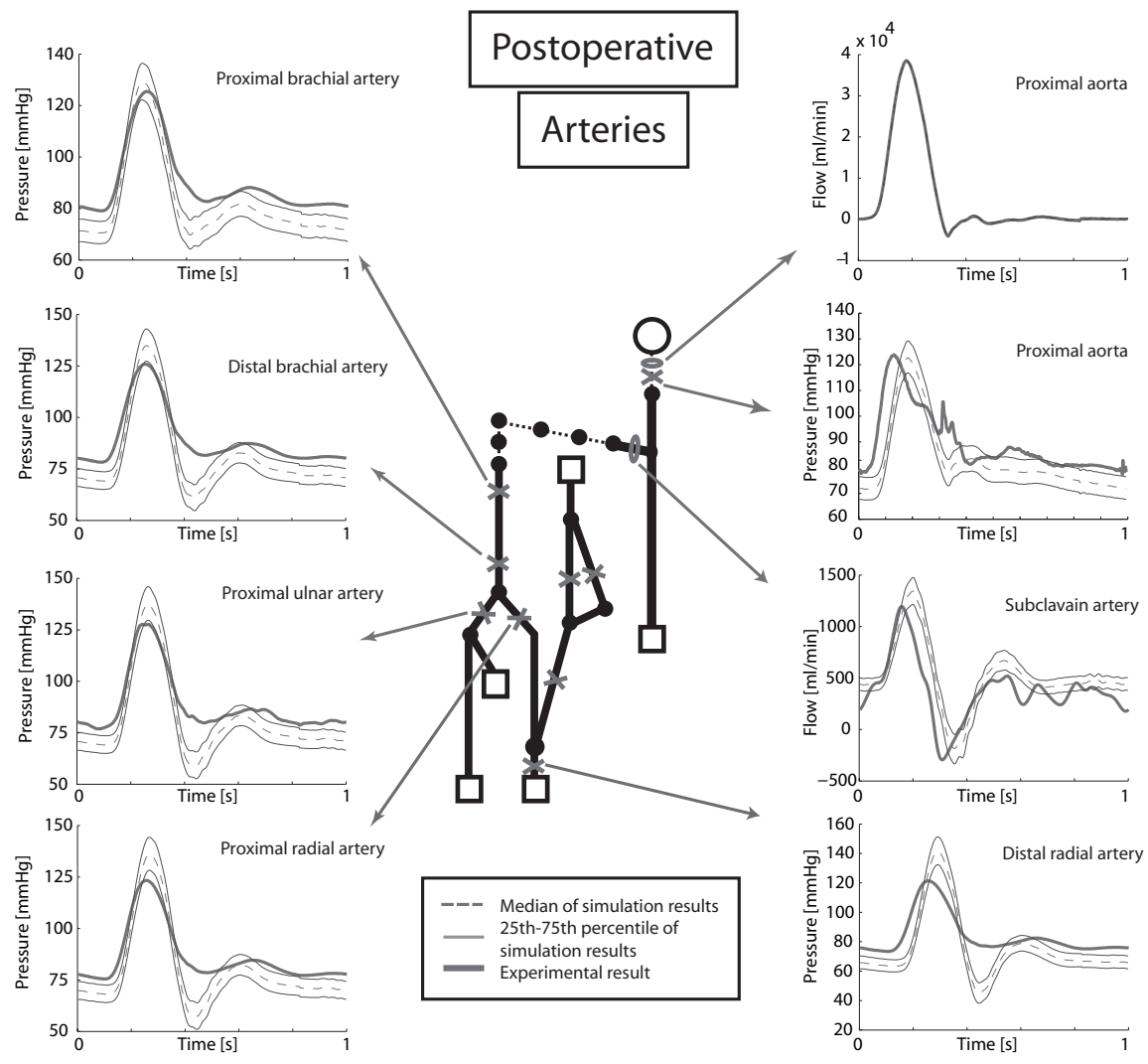

Figure 6.5: The postoperative pressure and flow waveforms on several arterial locations. 


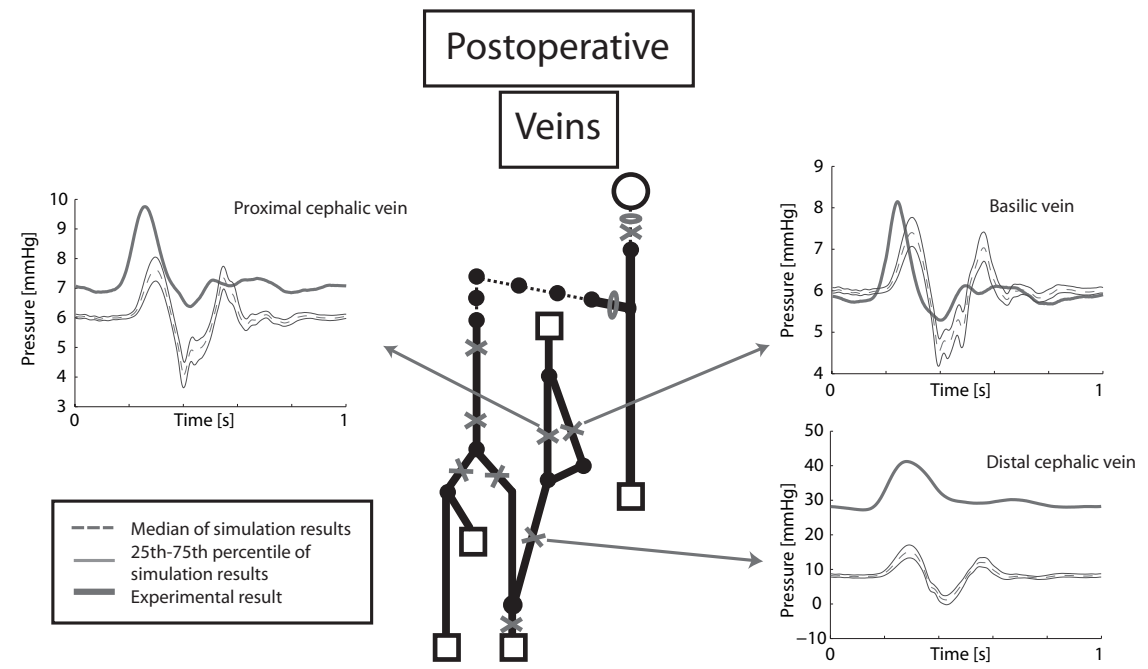

Figure 6.6: The postoperative pressure and flow waveforms on several venous locations.
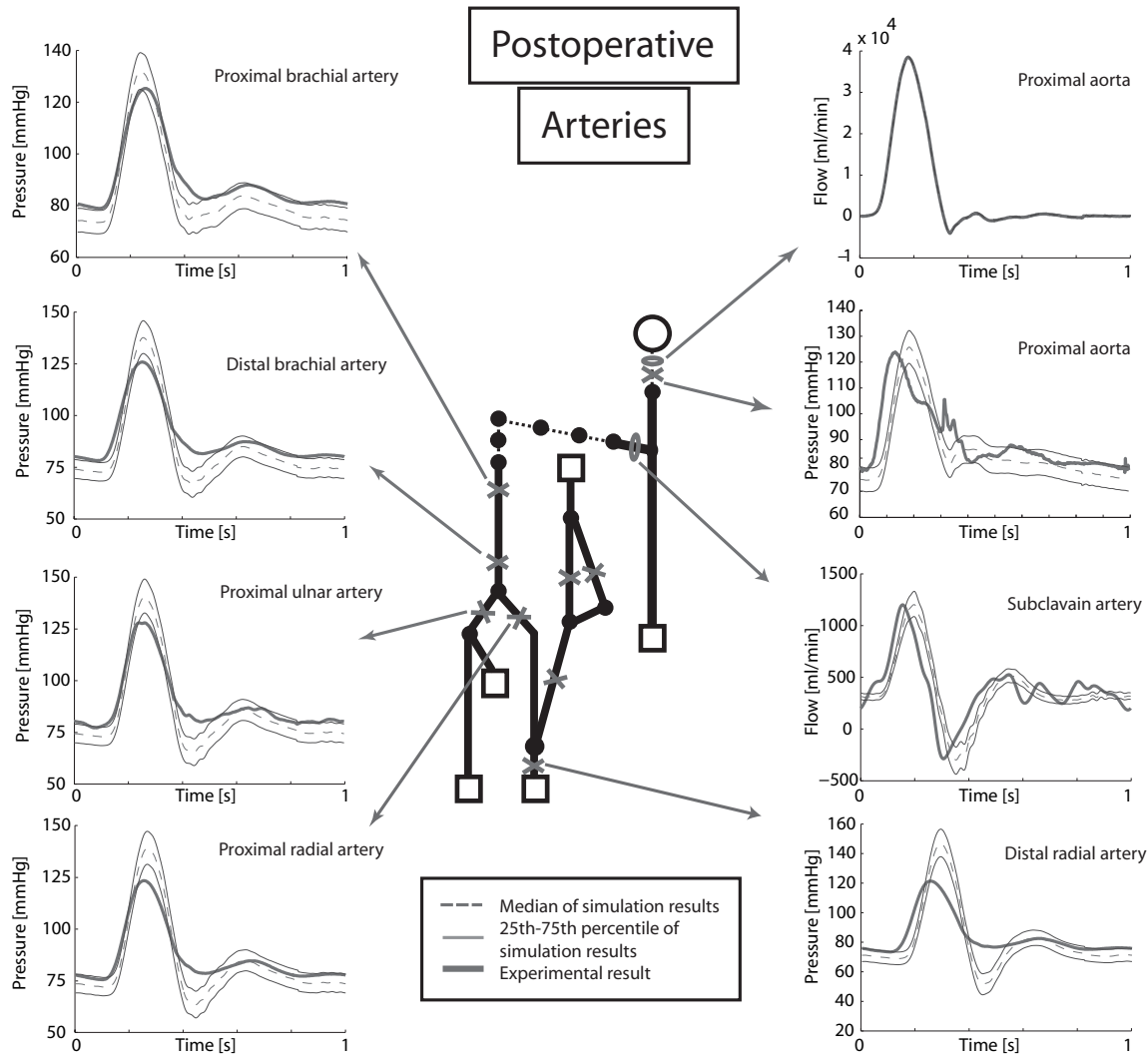

Figure 6.7: The postoperative pressure and flow waveforms on several arterial locations for simulations with additional resistance. 


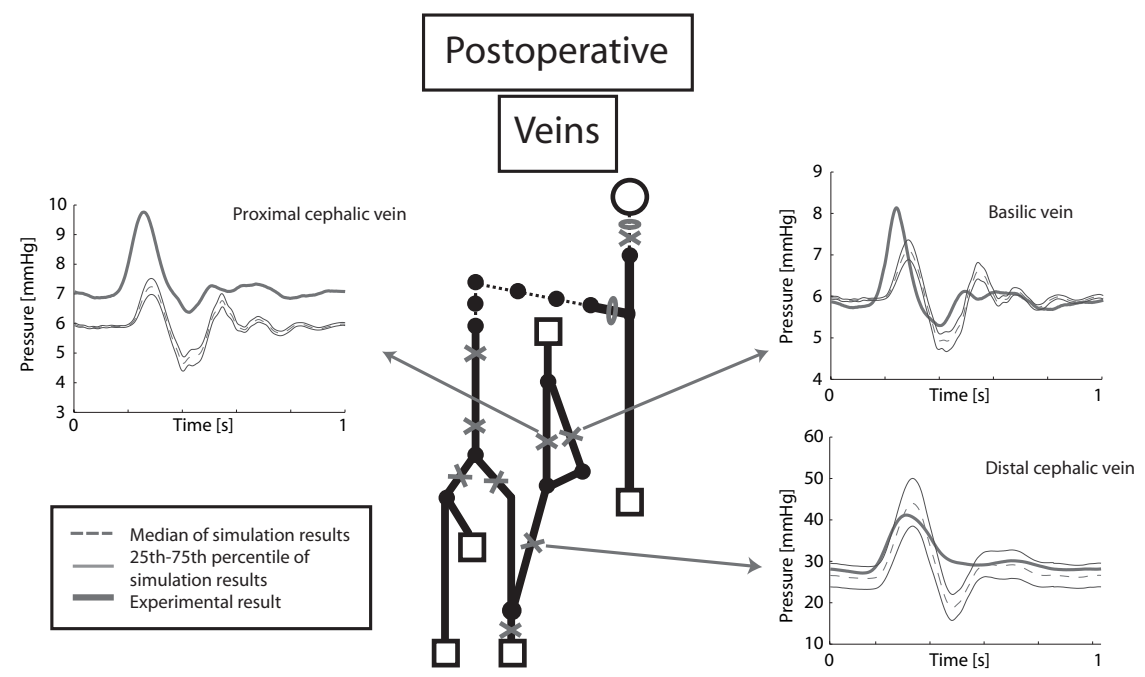

Figure 6.8: The postoperative pressure and flow waveforms on several venous locations for simulations with additional resistance.

\subsection{Discussion}

In this study, we aimed to experimentally corroborate a previously developed pulse wave propagation model for the prediction of pressure and flow before and after AVF creation. In this way it was determined if the physical description of the pulse wave propagation model in both cases was captured correctly by the model. It was found that the pulse wave propagation model was able to accurately simulate mean pressures and flows in the experimental setup but that the simulated pressure and flow waveforms were less attenuated than the measured waveforms. This most likely results from viscoelastic wall behavior of the experimental tubes $[63,130]$.

Experimental setups have previously been used to corroborate pulse wave propagation models, but not in the situation after AVF surgery when flows are significantly increased compared to normal and the anastomosis is included. Bessems et al. [63] have corroborated their pulse wave propagation model with both a straight and tapered viscoelastic silicone tube. Swillens et al. [127] used a silicone setup to corroborate their pulse wave propagation model in an aneurysm case study. An experimental setup that included a more detailed representation of the arterial tree and thus also physiological wave reflections resulting from bifurcations, was used by Matthys et al. [126]. Next to these experimental 
corroborations, Reymond et al. performed, recently, an in-vivo corroboration of a patientspecific pulse wave propagation model of the systemic arterial tree [65, 125]. However, although geometry, flow and pressure measurements were all performed on one individual patient, it was impossible to measure all input parameters patient-specifically (e.g. local vessel distensibility and peripheral resistance and compliance). Thereby, pressure and flow measurements are only possible on a limited number of positions over the systemic tree $[65,125,126]$. In addition, clinical measurements are usually hampered by large measurement uncertainties, which were not considered in any of the studies.

In contrast to these studies, the uncertainty of the model input was taken into account in this work, yielding an estimation of the precision on the pressure and flow waveforms. The $25^{\text {th }}$-to- $75^{\text {th }}$ percentile interval captured a decrease or increase ranging, dependent on location, from 2 to $14 \%$ of the median value for the mean pressures, while for the mean flows larger uncertainties were observed. Preoperatively, the uncertainties of the mean flows in the subclavian artery, the radial artery and the ulnar artery were respectively $60 \%, 20 \%$ and $30 \%$, while postoperatively the uncertainties of flows in these vessels were about $17 \%, 20 \%$ and $25 \%$. The uncertainty in mean flow in the distal cephalic vein was approximately $13 \%$. Considering precision is thus essential in corroboration studies. The large uncertainties in mean flows can be explained by the fact that the flow waveform oscillates around 0 which means that a small deviation in the waveform will result in a significant change in mean flow. Clinically, an uncertainty of approximately $20 \%$ in the mean flow in the subclavian/brachial artery is acceptable because the surgeon aims at an inflow of $400-500 \mathrm{ml} / \mathrm{min}$ while $300 \mathrm{ml} / \mathrm{min}$ is sufficient for proper hemodialysis treatment.

When simulating the postoperative situation, at first instance, it was observed that the subclavian artery flow waveforms had similar shapes but that the mean flow significantly differed between measurement and simulation. Furthermore, the simulated mean pressure in the distal cephalic vein was significantly smaller than the measured mean pressure. Upon closer examination, a large pressure drop from the distal cephalic vein to the proximal cephalic vein was found in the experimental setup. By excising the distal cephalic vein, a non-smooth lumen and significant twisting over the complete length was observed. Therefore, all simulations were repeated with a larger resistance over the distal cephalic vein. As a result, the correspondence between simulated and measured pressure and flow waveforms significantly improved. Thus, when incorporating the additional pressure drops resulting from structures such as twisting or kinking in the computational model, the model is able to simulate the postoperative situation adequately. For this, a clear picture of the vascular topology is required. 
The experimental setup constructed for this study allowed for model corroboration, since we were able to mimic flow enhancement after AVF creation and since all measured pressure and flow waveforms were comparable to physiological ones [99, 131]. The mean pressures and flows, both arterial and venous, simulated by the pulse wave propagation model, were adequately predicted and the overall shape of the pressure and flow waveforms were similar. However, the simulated waveforms were less attenuated than the measured ones, indicating that introduction of viscoelasticity might improve the computational model's capability to describe the physical phenomena. Previous studies $[65,126,127]$ already showed that a pulse wave propagation model is able to simulate pressure and flow waveforms in elastic arteries and that viscoelasticity improves the simulations [63, 125, 132, 133]. Since viscoelasticity is observed in human arteries [134, 135] including viscoelasticity in the model for patient-specific modeling might be required when the waveforms are of interest.

However, even after incorporating viscoelasticity into the pulse wave propagation model which results in a better description of the physical phenomena, clinical implementation might still be challenging. The reason for this is that the model needs to be adapted to patient-specific conditions which requires patient-specific model input parameters. Clinically, it is difficult to determine these parameters accurately due to limitations in measurement modalities or because the burden on the patient should be minimized. Fortunately, a sensitivity analysis can be applied to the model to determine which model input parameters are most important to assessed. Consequently, the number of required patient-specific measurements can significantly be reduced ([121]). Another challenge is including vascular adaptation (e.g. flow-mediated dilatation, maturation) and regulation (e.g. autoregulation of the peripheral bed) in the model. These physiological mechanisms should especially be introduced, when the (long-term) adaptation of a vascular access is of interest. The current model is only aimed at predicting mean flows directly after surgery.

\subsection{Conclusion}

The pulse wave propagation model was successfully corroborated with an experimental setup, mimicking pressure and flow changes following an AVF creation. Mean pressures and flows were adequately predicted and the overall shape of the pressure and flow waveforms were similar for the experiment and measurement. However, the experimental waveforms were more attenuated most likely resulting from neglecting viscoelasticity in the model. Furthermore, it was found that the uncertainties in model input parameters sig- 
Chapter 6. Model corroboration: An experimental study

nificantly influenced the output and should thus be taken into consideration in the analysis. 


\section{Chapter 7}

\section{Model corroboration: A clinical feasibility study}

This Chapter is based on:

W. Huberts, A.S. Bode, E.M.H. Bosboom, W. Kroon, W.P.M. van der Linden, R.N. Planken, F.N. van de Vosse, J.H.M. Tordoir, Patient-specific computational modeling of vascular access creation for supporting clinical decision-making, Submitted, (2011) 


\begin{abstract}
Patients with end-stage renal disease (ESRD) approaching the initiation of hemodialysis therapy are subjected to a preoperative vessel assessment to identify the most suitable site for arteriovenous fistula (AVF) creation. However, inadequate flow enhancement on the one hand, and excessive flow enhancement on the other hand, remain frequent complications, and hamper the use of the vascular access for hemodialysis therapy. In an effort to reduce these flow related complications, a patient-specific computational model, capable of predicting postoperative flow by considering multiple influencing factors simultaneously, has been developed. The purpose of this study was to determine the accuracy of the patient-specific model and to investigate its feasibility to support decision-making in AVF surgery.

Patient-specific pulse wave propagation models were created for 25 consecutive patients with ESRD awaiting their primary AVF creation. Input parameters for the models were obtained from clinical measurements and literature data. For every patient, a radiocephalic AVF, a brachiocephalic AVF, and a brachiobasilic AVF configuration were simulated and analyzed for their postoperative flow enhancement. The most distal configuration with a predicted flow between 400 and $1500 \mathrm{ml} / \mathrm{min}$ was considered to be the preferred location for AVF surgery. This suggestion of the model was compared to the choice of an experienced vascular surgeon. Furthermore, predicted flows were compared to measured flows one week after surgery.

In 4 patients computational modeling could not be performed due to sparse clinical data (3), or computational difficulties (1) and were therefore excluded from further analysis. Taken into account the confidence interval $\left(25^{\text {th }}\right.$ and $75^{\text {th }}$ percentile interval), overlap between predicted and measured postoperative flows was observed in $70 \%$ of the patients. Differentiation between upper and lower arm configuration was similar in $76 \%$ of the patients, whereas discrimination between two upper arm AVF configurations was more difficult. In 3 patients the surgeon created an upper arm AVF, while model based predictions allowed for lower arm AVF creation, thereby preserving proximal vessels. In one patient early thrombosis in a radiocephalic AVF was observed which might have been indicated by the low predicted postoperative flow.

Postoperative flow can be predicted relatively accurate and allows preoperative evaluation for multiple AVF configurations by using the described computational model. Computational modeling must therefore be considered a valuable additional tool in the preoperative work-up of patients awaiting vascular access creation.
\end{abstract}




\subsection{Introduction}

Patients suffering from end-stage renal disease (ESRD) depending on hemodialysis (HD) therapy require a functional vascular access (VA) [136]. This can be provided by creation of an arteriovenous fistula (AVF), creation of an arteriovenous graft (AVG), or the insertion of a central venous catheter (CVC). Since the use of prosthetic graft material (AVG and $(\mathrm{VC})$ is associated with reduced patency rates and higher mortality rates [137, 138], guidelines advocate the use of native vessels for VA creation [9]. However, an important downside of AVF creation is the significant probability of early thrombosis or nonmaturation (20-50\%) due to insufficient flow enhancement, particularly in lower arm fistulas $[6,10]$, and excessive postoperative flow enhancement resulting in steal syndrome and cardiac failure (up to 20\%) in elbow fistulas [12, 13]. In an effort to limit these complications, an extensive preoperative duplex ultrasound (DUS) evaluation of the upper extremity vascular tree is performed to select the most suitable site for AVF creation [20]. Unfortunately, flow related complications persist and additional interventions are often needed to make the AVF suitable for HD treatment [18].

To decrease the incidence of flow related complications, preoperative prediction of postoperative VA flow would be beneficial. Patient-specific computational modeling has the potential to predict this postoperative VA flow by using physical laws for quantitative integration of multiple prognostic factors (e.g. vascular diameters, arterial compliance). Besides, computational modeling has already proved to be of assistance in aortic aneurysmal disease [139, 140], in cerebral disease [141, 142], and coronary artery disease [143, 144]. Although models have been used previously to gain insight in VA hemodynamics and pathologies, or disease progression associated with it [145, 146, 147], predictive models, aiming for a more accurate risk-estimation regarding the development of flow related complications, have not been used.

Within the $7^{\text {th }}$ Framework Program of the European Commission (i.e. the ARCH ICT224390 project), a pulse wave propagation model was developed that is to be implemented in the preoperative work-up of patients awaiting VA creation, thereby providing additional preoperative information to the surgeon. A pilot study on a limited number of patients showed that such a model, when adapted to patient-specific conditions, has the potential to suggest the most suitable AVF configuration by predicting postoperative flow [101]. However, to personalize the model, a considerable number of input parameters needs to be obtained, which are subjected to biological variations and measurement inaccuracies. These input uncertainties will result in uncertainties in flow predictions, and may hamper clinical implementation of the model. 
In this study, the feasibility and accuracy of the pulse wave propagation model to support decision-making in AVF surgery was examined.

\subsection{Material and methods}

\subsubsection{Study population}

Twenty-five consecutive patients suffering from ESRD awaiting their first VA creation were enrolled in this prospective observational study. The study was approved by the local medical ethical committee, and written informed consent was obtained from all individuals prior to enrolment in the study.

\subsubsection{Pulse wave propagation model}

The pulse wave propagation model used here, has been described in detail in previous work [101]. In short, the model simulates pressure and flow waveforms on several arterial and venous locations of the upper extremity. Depending on the site (left or right) and the AVF configuration (radiocephalic AVF (RCAVF), brachiocephalic AVF (BCAVF), brachiobasilic AVF (BBAVF)), inflow arteries and outflow veins were included in the computational domain (Figure 7.1). Each vessel of the computational domain was divided into segments with a maximum length of $5 \mathrm{~cm}$, describing the local relation between pressure and flow via a lumped parameter approach. Such a lumped segment consists of a resistor $R$, representing the viscous resistance to blood flow through the vessel segment, a resistor $R_{L}$, representing the resistance to blood flow through small side-branches not modeled in detail, an inductor $L$, representing the inertia of the blood and a capacitor $C$, representing the vascular compliance (i.e. storage capacity of the vessel). For the anastomosis, a segment was developed consisting of nonlinear resistors that depend on anastomosis angle and blood flow. Arteries not included in the computational domain as well as the peripheral vascular beds were modeled by windkessel elements with a specific resistance and compliance. As boundary conditions, an intravenous pressure was prescribed at the subclavian vein, whereas an inflow was prescribed at the aorta. Since the latter is preoperatively unknown, aortic flow was measured and iteratively updated by scaling the preoperative waveform until the mean aortic pressure was restored to the preoperative level. All other preoperative model parameters (e.g. peripheral resistances) were kept constant (Figure 7.2). 

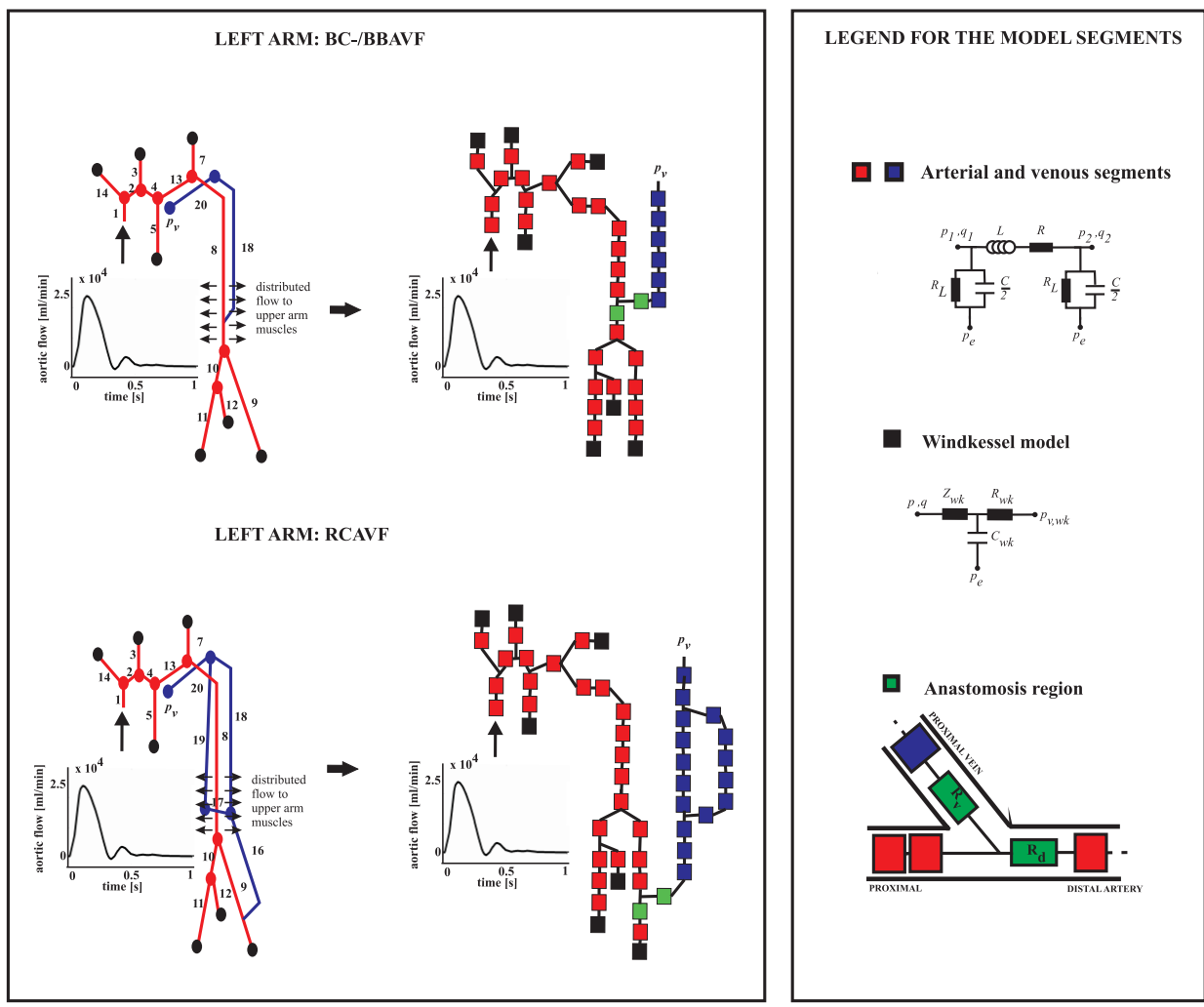

Figure 7.1: The left arm vasculature of interest (computational domain, left) divided into arterial, venous and anastomosis segments (middle). These segments locally describe the relation between pressure $p$ and flow $q$ via a lumped parameter approach (right) and consists of a resistor $R$ (viscous resistance to blood flow), a resistor $R_{L}$ (viscous resistance of blood flow to small side-branches), an inductor $L$ (blood inertia) and a capacitor $C$ (vascular compliance). The anastomosis is modeled with two nonlinear resistors $R_{v}$ and $R_{d}$. The windkessels consist of two resistors, $Z_{w k}$ and $R_{w k}$ (together the peripheral resistance) and a capacitor $C_{w k}$ (peripheral compliance). This figure is adapted from Huberts et al. [101]. 


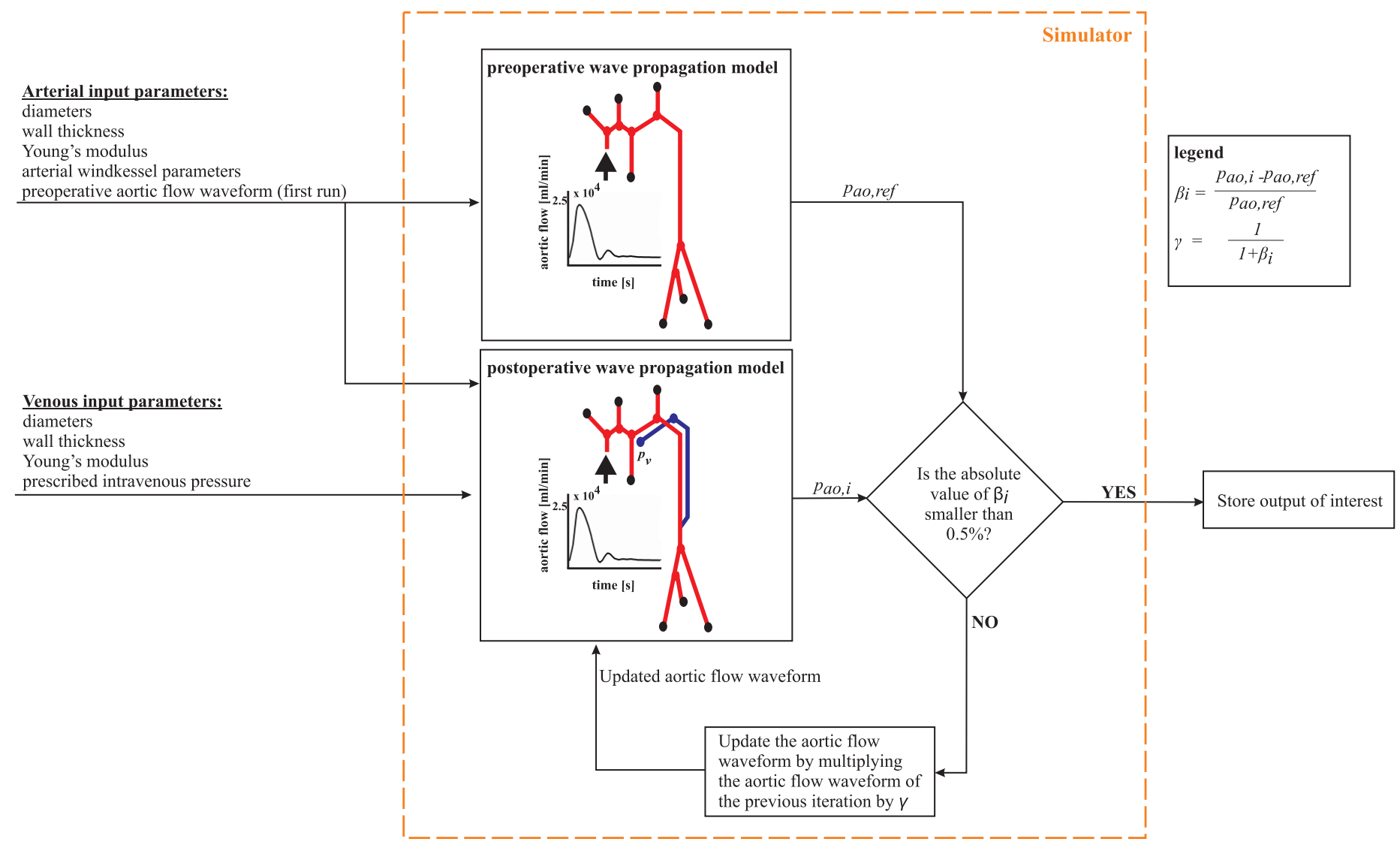

Figure 7.2: Iterative scheme that describes the estimation of the aortic flow after AVF creation. 


\subsubsection{Personalization of the pulse wave propagation model}

To personalize the input parameters of the pulse wave propagation model, patient-specific anatomy (vessel length, vessel diameters, vessel wall thickness) and mechanical characteristics of the vessels (vascular compliance) were mandatory. Furthermore, information on anastomosis configuration (location, angle), windkessel parameters, blood properties (density and dynamic viscosity), intravenous pressure and aortic flow waveform were required. However in clinical practice, it is impossible to assess all these parameters for every patient. Fortunately, not all parameters are equally important for the prediction of postoperative flow enhancement; model parameters that need to be measured opposed to model parameters that can be estimated from literature, were identified previously in a sensitivity analysis [148].

Model parameters were therefore chosen as follows. Arterial lengths are based on a generic geometry taken from Stergiopulos et al. [25]. Venous lengths are considered equal to arterial lengths on the same anatomical location. Upper extremity vascular diameter measurements were obtained patient-specifically on discrete locations by performing an extensive duplex ultrasound examination which is described in detail in Bode et al. [122]. Prior to the venous measurements, a tourniquet was applied to induce venous dilatation and this resulted in reproducible venous diameter measurements [94]. Missing diameters of the arm vasculature were obtained by linear inter-, or extrapolation. Diameters of the aorta and its primary branches were based on literature and scaled according to upper extremity arterial diameters [25]. Vessel wall thicknesses were derived from wall thickness-to-radius ratios: a ratio of $15 \%$ was used for the subclavian, axillary and brachial artery, whereas a ratio of $20 \%$ was used for the radial, ulnar and interosseus artery $[24,95,96]$. The ratios of all other arteries were based on literature [25]. For veins a ratio of $10 \%$ was chosen [149].

Mechanical properties of the upper extremity vessels were characterized by vascular compliance. For this, in addition to wall thickness and diameter, the Young's modulus was required $[36,101]$. The Young's modulus of the brachial artery was determined via arterial distensibility, which was assessed by a Picus ultrasound machine equipped with ARTLAB software (ESAOTE, Maastricht, The Netherlands). For each patient, vessel wall distension over the cardiac cycle was measured using a wall-tracking technique in combination with continuous, non-invasive pressure registration (Nexfin, BMEye, Amsterdam, The Netherlands). The Young's modulus of the brachial artery was applied for the compliance of all arterial arm segments, whereas for the aorta and veins Young's moduli were based on literature [25, 101]. 
The location of the anastomosis was set to $5 \mathrm{~cm}$ proximal to the wrist in case of a lower arm AVF and $5 \mathrm{~cm}$ proximal to the elbow bifurcation in case of an upper arm AVF. The angle of the AVF between the proximal artery and the vein was set to 45 degrees.

Windkessel parameters were personalized by using mean arterial pressure and mean arterial flows in the aorta, brachial, radial, and ulnar artery. Mean flows were obtained by MR flow measurements (Philips Healthcare, Best, The Netherlands). The preoperative aortic flow waveform was prescribed at the first aortic node.

Blood was considered to behave as an incompressible Newtonian fluid with a density of $10^{3} \frac{\mathrm{kg}}{\mathrm{m}^{3}}$ and a dynamic viscosity of $3 \cdot 10^{-3} \mathrm{~Pa} \cdot \mathrm{s}$. Intravenous pressure at the subclavian vein was set to $10 \mathrm{mmHg}$ [99].

An extensive description of the patient-specific measurement protocol can be found in Bode et al. [122].

\subsubsection{Analysis}

For every patient, three different AVF configurations were considered (RCAVF, BCAVF, BBAVF), and evaluated with respect to their postoperative flow directly after surgery. In lower arm fistulas the immediate postoperative flow is approximately $60-70 \%$ of the flow after successful maturation, while in an upper arm AVF this is already almost $80 \%$ [32]. Furthermore, postoperative flows larger than $30 \%$ of the cardiac output are associated with an increased risk for cardiac failure and hand ischemia $[12,13]$. As a result, AVF configurations resulting in a predicted postoperative flow between 400 and $1500 \mathrm{ml} / \mathrm{min}$ were considered by the model as an option for VA creation. When more VA configurations resulted in a flow exceeding $400 \mathrm{ml} / \mathrm{min}$, the sequential order of preference was RCAVF, BCAVF, and BBAVF. To objectivate the model's capability to identify the optimal location for AVF creation, the suggested AVF configuration was compared with the choice of a surgeon with ample experience in VA surgery (more than 1000 AVF creations). The choice of the surgeon was based on clinical experience and preoperative mapping of upper extremity vasculature with DUS.

Subsequently, in order to quantitatively determine the accuracy of flow predictions, predicted flows of the created AVF configuration were compared with observed postoperative flows as measured with DUS one week after surgery. In this perspective, the uncertainty of the flow prediction resulting from input parameter uncertainty (summarized in the Appendix) is evaluated by means of Monte Carlo simulations as described by Huberts et al. [148], and expressed through the $25^{t h}-75^{\text {th }}$ percentile interval. The Monte Carlo simulations were also used for a global sensitivity analysis to examine the effect of assumptions 
that were made during the personalization of the model parameters.

\subsection{Results}

\subsubsection{Vascular access configuration}

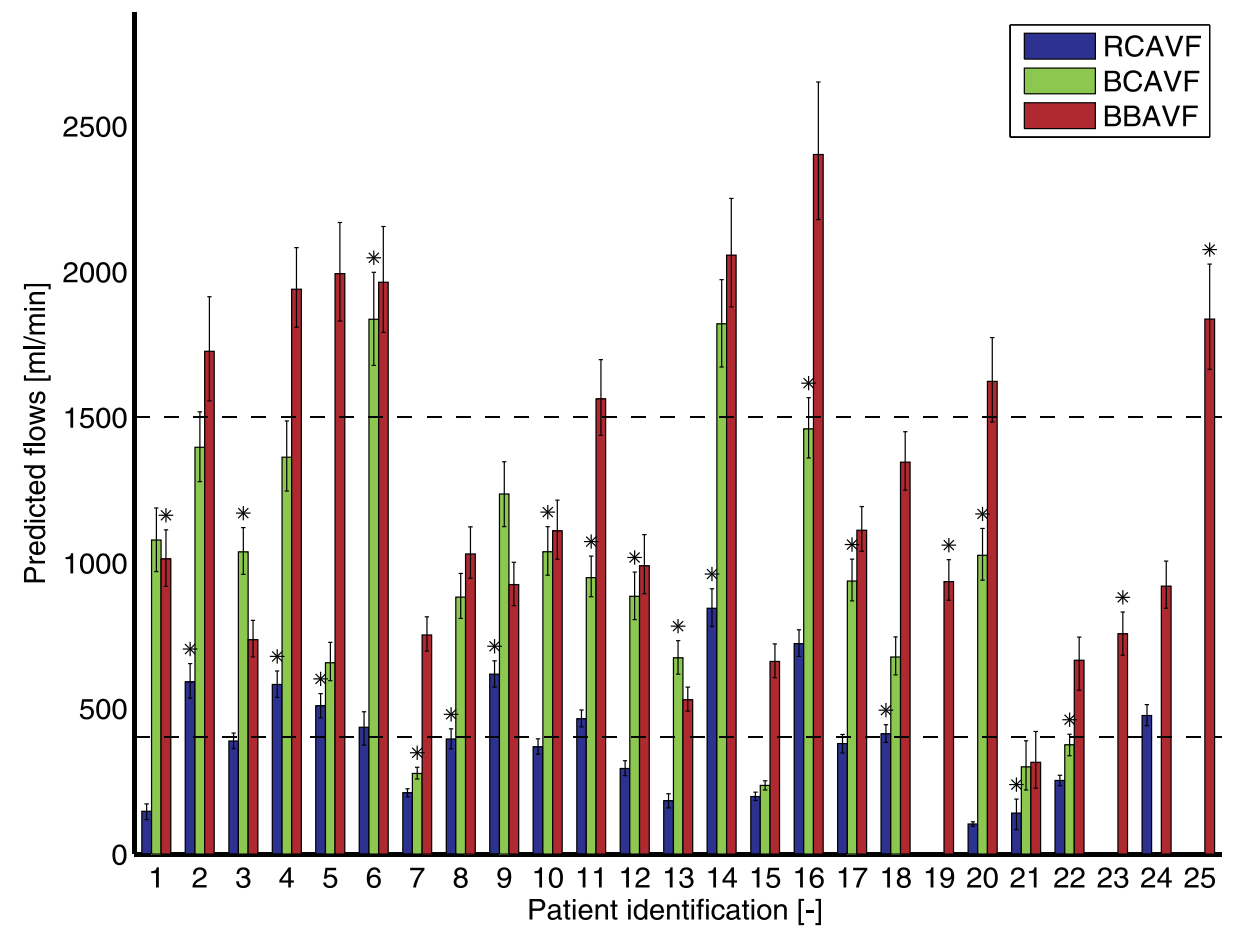

Figure 7.3: The predicted postoperative flows for a RCAVF, BCAVF and BBAVF configuration. The flows are presented as the median of all Monte Carlo simulations with their $25^{\text {th }}$ and $75^{\text {th }}$ percentile interval. In 4 patients postoperative brachial artery flow could not be simulated for all three AVF configurations because essential patient-specific data were missing due to thrombosis of the cephalic vein (patient \#19, \#23, and \#25) or because the computations did not converge for all Monte Carlo simulations (patient \#24).

In 4 of 25 patients postoperative brachial artery flow could not be simulated for all three AVF configurations because essential patient-specific data were missing due to thrombosis of the cephalic vein (patient \#19, \#23, and \#25) or because the computations did not 
converge for all Monte Carlo simulations (patient \#24). As in these patients predicted flows for the different AVF configurations could not be compared they were excluded from the analysis.

In the remainder of patients, the model suggested an upper arm or lower arm AVF configuration in agreement with the choice of the surgeon for 16 patients (16/21: 76\%). In five patients the suggestion of the model and the choice of the surgeon were different (Figure 7.3); in patients \#6, \#11, and \#16 model predictions may have allowed for a lower arm AVF, while the surgeon created upper arm AVFs. Conversely, in patient \#8 the surgeon created a lower arm AVF, while the model suggests to create an upper arm AVF. Also in patient \#21 a lower arm AVF was created, whereas the model predicts a too low postoperative flow for all configurations. These low flow predictions might have been indicative for the early failure as observed in this patient.

When differentiating between RCAVF, BCAVF, and BBAVF, the model suggests the same AVF configuration as the surgeon in 12 patients (12/20: 60\%) (Figure 7.3). For this analysis, one additional patient (\#15) was excluded, because during the surgical procedure immediate thrombosis occurred for the intended BBAVF configuration and prosthetic graft material was used to create the VA. In addition to the five previously mentioned patients, there is a discrepancy between the suggested configuration of the model and the choice of the surgeon with respect to a BCAVF or BBAVF configuration in three patients (\#1, \#7, and \#22).

\subsubsection{Absolute postoperative flow prediction}

Postoperative flow predictions could be compared to clinically measured flows in 23 of 25 patients. One patient (\#15) was excluded from the analysis because graft material was used for creation of the VA conduit, which is not supported by the computational model. A second patient (\#21) was excluded because of immediate thrombosis and, as a result, no postoperative flow measurements were available.

Figure 7.4 shows predicted flows versus measured flows one week after surgery. In addition, the flow measurement at six weeks is visualized to gain insight into flow enhancement during the maturation phase. At one week, predicted and measured flow show overlap in 16 patients $(16 / 23$ : $70 \%)$. In patient \#1, \#6, \#23, and \#24 the predicted flow is an overestimation of the measured flow, while in patient \#7, \#18, and \#22 the predicted flow is an underestimation of the measured flow. In patient \#1 a significant hematoma was identified during the immediate postoperative duplex control, whereas in patient \#23 postoperative thrombosis was observed one week after surgery. 


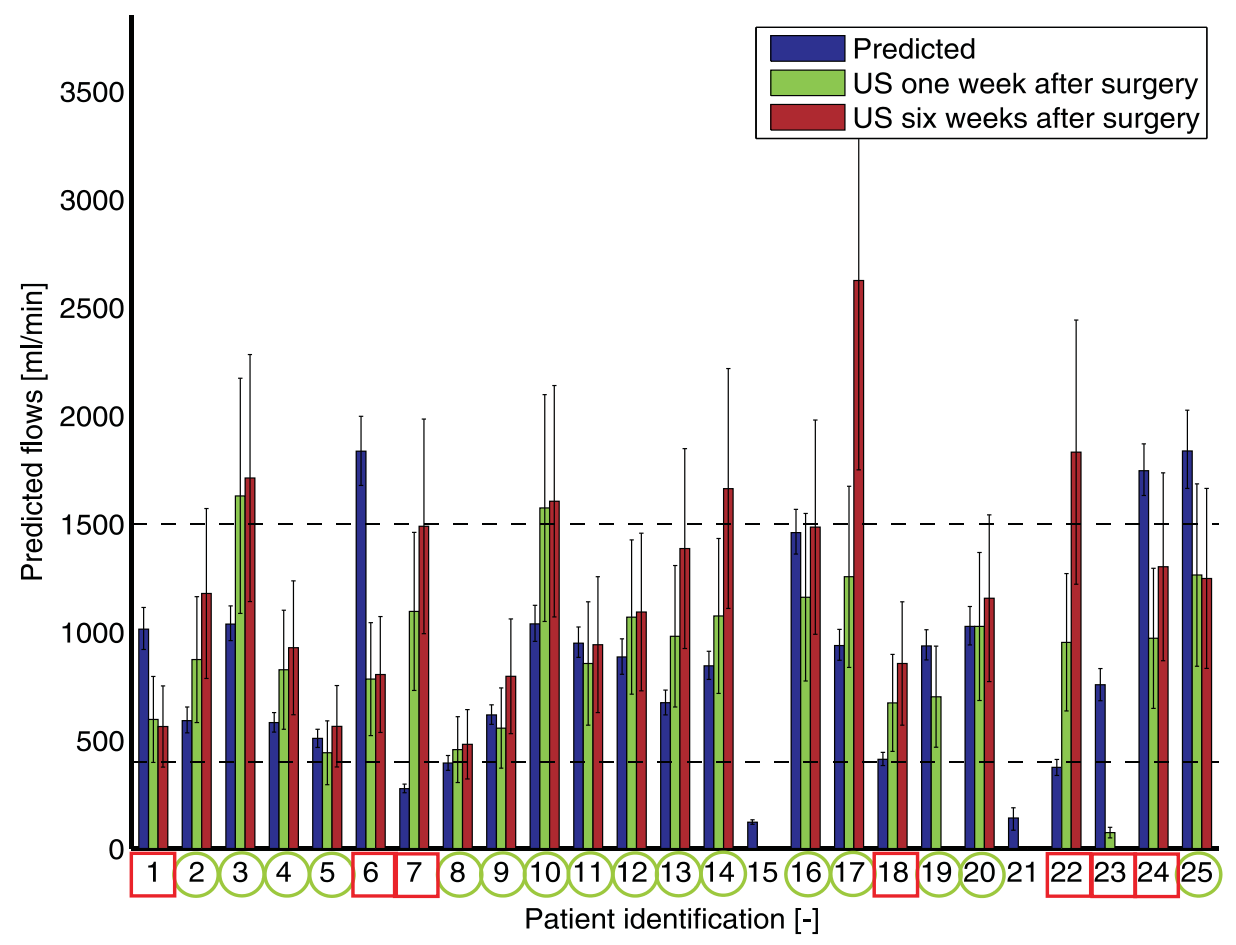

Figure 7.4: Schematic visualization of predicted and measured postoperative flows for the AVF configuration created by the vascular surgeon. The error bars in predicted flow are the result of inaccuracies in input parameters, while the error bars in postoperative flow are the result of measurement inaccuracies [101]. For patient \#15 prosthetic graft material was used for VA creation. For patient \#21 no postoperative flow measurements could be obtained due to immediate thrombosis. A green circle around the patient identification represents overlap between predicted and measured postoperative flow (16 patients). A red square around the patient identification represents a discrepancy between predicted and measured postoperative flow (7 patients).

\subsubsection{Sensitivity analysis}

The sensitivity analysis showed that the effect of assumptions made during model personalization (vessel length, arterial scaling for aortic diameter, determination of wall thickness and Young's modulus), were negligible compared to the effect of the uncertainty in measured input parameters (e.g. diameter of the upper extremity vasculature and the peripheral resistances), which was expected based on an earlier sensitivity analysis [148] of the model and previous clinical studies [20, 21]. 


\subsection{Discussion}

In this study we investigated the feasibility and accuracy of a pulse wave propagation model to support decision-making in AVF surgery by predicting postoperative brachial artery flow for multiple AVF configurations. By using the model described, postoperative brachial artery flow can be estimated relatively accurate without subjecting the patient to excessive additional preoperative measurements.

In clinical routine, preoperative mapping of upper extremity vasculature with DUS is considered the method of choice to identify the most suitable site for VA creation, and its clinical implementation has been associated with a significant reduction of postoperative failure rates $[150,151]$. Nevertheless, complications related to either insufficient flow enhancement, particularly in lower arm fistulas, and to excessive flow enhancement, mainly in upper arm fistulas, remain responsible for VA failure in a significant number of patients $[6,10,12,13]$. Therefore, alternative modalities to decrease the incidence of flow related complications have become of interest. Prior work of our group focused on the development of a computational tool in which pressure and flow distributions in the upper extremity vasculature can be simulated [101]. In this way, hemodynamic consequences of AVF creation can be evaluated by taking multiple prognostic factors, as well as their complex interplay into consideration, instead of focusing on diameter measurements on a finite number of discrete locations.

Pulse wave propagation models have been shown in both experimental setup and in-vivo to be able to simulate pressure and flow waveforms on multiple locations [63, 125]. Some studies have reported the use of pulse wave propagation models for prediction of outcome after vascular surgery $[50,51]$. However, the application of predictive models is still in its infancy and has, to our knowledge, not been examined for application in AVF surgery planning.

By using a pulse wave propagation model in this study, overlap between predicted and observed postoperative flow was observed in $70 \%$ of the patients. Predicted flows of 4 patients overestimate the flows measured with ultrasound at one week, while the predicted flows of 3 patients underestimate the measured flow. Overestimation of flow in patient \#1 and \#23 might be explained by the occurrence of a postoperative hematoma compressing the venous outflow trajectory, and by unexpected early thrombosis, respectively. Other possible explanations for overestimation of postoperative flow might be stenotic segments, curvature or kinking not detected with routine DUS examination. These vessels abnormalities are not included in the computational model but would increase the resistance to blood flow in the VA conduit. A possible solution might be to perform a 
MRA of the upper extremity vasculature which allows for assessment of vascular diameter over the complete vascular trajectory, and for the identification of stenoses, curvature and kinking [152]. In addition, MRA might be beneficial in patients in whom vascular anatomy has been influenced by previous VA creation. The model can easily be adapted to deal with resulting extra pressure drops and altered vascular geometries. Underestimation of postoperative flow might be caused by the lack of vascular adaptation laws in the computational model. In patient \#7 and \#22, this might be indicated by the large flow enhancement from week one to week six after AVF creation. In addition, flow-mediated dilatation and autoregulation are not yet incorporated in the model.

According to the observations in the current study, the suggestion of a patient-specific wave propagation model for an upper or lower arm AVF configuration corresponds to the selection of the surgeon in $76 \%$ of the patients. In three patients the surgeon decided to create an upper arm AVF, whereas the model allowed for creation of a lower arm AVF. When this additional information would have been available to the surgeon at the time of VA planning, the surgeon might have considered to preserve proximal vessels for future VA procedures by primary creation of a lower arm AVF. Conversely, upper arm AVF creation was suggested in two patients while a lower arm AVF procedure was performed. In one of these patients, immediate thrombosis was observed, while in the other patient the measured flow was slightly above the threshold of $400 \mathrm{ml} / \mathrm{min}$, as was predicted by the model. This additional information might also have changed the surgeon's choice when known in advance. In this perspective, computational modeling should be considered a potential valuable tool in the preoperative work-up, in addition to the currently performed diameter measurements. However, a randomized trial is required to establish the additional value of the model in routine clinical practice.

Considering the differentiation between BCAVF and BBAVF, a discrepancy between model and surgeon was observed in three patients: two BCAVFs were created where the model suggested a BBAVF configuration, and one BBAVF was created while a BCAVF appeared to be feasible according to the model. Although the model might already be able to differentiate between an upper and lower arm AVF, it appears to be more difficult to distinguish between two upper arm AVF configurations. The most likely explanation for these inconsistencies is the uncertainty in venous input parameters and, possibly, the neglection of accessory veins and vascular adaptation in the model.

The study presented here has limitations. Firstly, a relatively small number of patients was enrolled. However, for assessing the feasibility of the model as well as evaluating the impact of model input uncertainties on the output, the number of enrolled patients suffices. Nonetheless, to determine the predictive value, sensitivity and specificity of the 
model as additional tool in the preoperative work-up in patients awaiting VA creation, a large randomized clinical trial needs to be initiated. As a further limitation, one might consider the difficulty of model personalization, since in clinical practice not all input parameters can be obtained for each patient with sufficient accuracy (e.g. windkessels). The reason for this is the limited availability of measurement modalities, and moreover, because the additional burden on the patient should be minimized. Fortunately, the previous sensitivity analysis showed that some input parameters are more important than others [148]. As a result, half of the input parameters could be derived from literature. To this end assumptions had to be made, for which an additional sensitivity analysis in this study showed, that these assumptions do not significantly alter outcome.

In conclusion, we have demonstrated that a patient-specific pulse wave propagation model can be considered potentially beneficial in the preoperative work-up of patients awaiting VA creation, since postoperative flow can be predicted relatively accurately for multiple AVF configurations. Future effort should focus on acquiring a more detailed overview of patient-specific vasculature to capture vascular pathology and geometry, and for simulation of the maturation process, adaptation laws should be incorporated in the model. To establish the additional value of modeling in clinical decision-making, a large prospective randomized clinical trial needs to be performed. 


\section{Appendix: Uncertainty domains}

Monte Carlo simulations (see Robert et al.[128] for details about Monte Carlo simulations) are used to determine the uncertainty in model predictions resulting from uncertainty in the input parameters assessed by measurements or from literature by applying certain assumptions. The uncertainties in the model parameters and their motivation are shown below.

Table 7.1: Measured and assumed parameters and their uncertainties.

$\begin{array}{lcc}\text { Measurements } & \text { Uncertainty } & \text { Based on } \\ \text { vessel diameters upper extremity } & \pm 10 \% & {[72,107]} \\ \text { brachial distensibility } & \pm 20 \% & {[72]} \\ \text { brachial pressure } & \pm 10 \% & {[110]} \\ \text { axillary, radial and ulnar artery flow } & \pm 15 \% & {[109]} \\ \text { mean aortic flow } & \pm 10 \% & {[109]} \\ \text { Assumptions } & & \\ \text { vessel lengths } & \pm 10 \% & \text { Demographical data (http://www.cbs.nl) } \\ \text { vessel diameter scaling factor } & \pm 20 \% & \text { Retrospective patient cohort } \\ \text { position of diameter measurements } & \pm 3 \mathrm{~cm} & \text { Clinical experience } \\ \text { wall thickness-radius ratio } & \pm 40 \% & {[95]} \\ \text { generic arterial Young's modulus } & \pm 20 \% & {[72]} \\ \text { generic venous Young's modulus } & \pm 20 \% & \text { Assumed equal to arterial Young's modulus } \\ \text { anastomosis position } & \pm 2 \mathrm{~cm} & \text { Clinical experience } \\ \text { anastomosis angle } & \pm 20 \% & {[30]} \\ \text { time constants } & \pm 30 \% & {[72]} \\ \text { intravenous pressure } & \pm 50 \% & {[1,99]} \\ \text { generic mean windkessel flow } & \pm 15 \% & {[149]}\end{array}$


Chapter 8

\section{General discussion}




\subsection{Motivation, aim and methodology}

Adequate hemodialysis therapy is impossible without a well-functioning vascular access that serves literally as lifeline for the patients [7, 8, 9]. An arteriovenous fistula (AVF), which is a permanent surgically created connection between an artery and vein (anastomosis) in the upper extremity [7, 8, 9], is the preferred vascular access. The AVF can be created at several locations and the most suitable site for AVF creation is a compromise between complication rates, long-term patency and saving potential sites for future vascular access $[11,12,13,14]$. To select the optimal site for AVF creation preoperatively, the vascular surgeon gathers as much information as possible regarding vessel caliber and continuity, possible stenoses and significant side-branches by using a physical examination, a duplex ultrasound examination and his clinical experience. AVFs are preferably created at wrist level (RCAVF) because in that case proximal locations are preserved for future vascular accesses. Moreover, in general, postoperative flows are smaller than in upper arm AVFs (BCAVF, BBAVF) resulting in less long-term complications like cardiac failure and distal ischemia [7, 12, 13, 14]. But lower arm AVFs are hampered by early thrombosis or non-maturation which results in insufficient postoperative flow $[6,7,8,10]$. Since all complications are associated with postoperative flow [10, 13, 14, 32], a preoperative quantitative measure of the postoperative flow would provide the surgeon with additional information which might improve the clinical decision-making in AVF surgery planning. In addition, distal ischemia is associated with a low systolic pressure distal to the anastomosis $[7,8,9]$ and also for this parameter a preoperative measure can be beneficial. Unfortunately, these hemodynamic parameters are affected by multiple factors (e.g. vessel geometry, vessel mechanics, anastomosis resistance) and differ between patients. A patient-specific computational model combines the influencing factors by means of physical laws and might have the potential to predict postoperative hemodynamics and, more specifically, postoperative flow and distal systolic pressure.

Up till now computational models describing hemodynamics have mostly been used to get insight into (patho-)physiological phenomena [25, 33, 34, 37, 55]. Using these models for predictive surgery is still in its infancy and requires that these models are fed by patient-specific data to personalize them. However, the number of input parameters that can be measured in clinical routine is limited. Patient data are thus compromised by uncertainty leading to uncertainties in model predictions. This implies that a balance must be found between model complexity and uncertainty in the model parameters to minimize the uncertainty in the predictions.

In this thesis, we developed a computational model that is able to predict postoperative 
flow and distal systolic pressure after AVF creation. In addition, its applicability to support clinical-decision making in vascular access surgery was examined. To this end, three steps were executed: model development, model personalization and model corroboration. Model corroboration is here defined as the qualitative and quantitative validation of the model.

\subsection{Computational model development}

A pulse wave propagation model was chosen to be the most appropriate computational model for our application, because the distribution of pressure and flow throughout the whole vascular system was of interest. Detailed information regarding local velocity fields, which can be obtained by three-dimensional models, were not the primary aim here. An advantage of this approach is that pressure and flow waveforms can be calculated within minutes which facilitates clinical application [50, 55]. Moreover, pulse wave propagation models are easier to adapt to patient-specific conditions than threedimensional models $[50,55]$. The computational domain of the pulse wave propagation model consisted of the complete vascular access conduit including the major inflow arteries, outflow veins and the anastomosis.

We have chosen for a distributed lumped parameter implementation of the pulse wave propagation model of Bessems [61] so that additional coupling equations for bifurcations and merging vessels were not required as the coupling is implicitly done during model assembly [153]. This also simplified the implementation of a nonlinear segment for the anastomosis. Furthermore, the lumped parameter implementation showed to be robust and able to deal with a large variety in inputs and imperfect clinical datasets.

The derived lumped parameter pulse wave propagation model consists of serially connected lumped segments that locally describe the relation between pressure and flow in a tapered or untapered vessel segment. The lumped segments were derived in Chapter 2 and based on the 1D mass and momentum equations derived by Hughes and Lubliner [59] after neglecting the convection term and assuming a Womersley number dependent approximate velocity profile to get a proper estimate for the wall shear stress [61]. The lumped segment consists of a Womersley number dependent resistor and an inductor arranged in parallel, respectively representing the flow impedance in respectively the vessel core and the boundary layer, in series with a second Poiseuille resistor. Two capacitors were added in a $\pi$-configuration, in analog to [36], to capture vessel compliance. The proposed lumped segment simplified the electrical analog of Jager et al. [64] who added extra constant inductors and resistors. It was shown that, for arbitrary Womersley num- 
bers, the relative impedance of Womersley theory [84] was accurately approximated by the lumped segment. For small and large Womersley numbers, the relative impedances of the lumped segment coincided with Womersley theory.

Since the resulting lumped segment is frequency dependent, it needs to be evaluated in the frequency domain. However, for our application of AVF surgery nonlinear segments are required, e.g. for the anastomosis. Hence a frequency domain analysis was no longer possible and we chose for a time domain analysis in which the lumped segment was evaluated, in analog to [61], only for the Womersley number corresponding to the characteristic frequency of a heartbeat. As a result, the arrangement of the elements in the lumped segment was slightly changed to better represent Womersley theory. These adapted lumped segments were used in Chapter 3 to construct the lumped parameter pulse wave propagation model. Our lumped parameter implementation of the pulse wave propagation model was benchmarked with the spectral element implementation of [61] and we observed similar simulated pressure and flow waveforms, demonstrating a correct realization of the numerical scheme.

The pulse wave propagation model that was developed was applied to AVF surgery planning and it was shown that the model was able to capture the pressure and flow changes after AVF creation that are also observed in literature [99], i.e: a significant increase in brachial artery flow, a drop in arterial pressure and disappearance of the preoperative brachial artery backflow. These observations were more pronounced in upper arm AVFs which is also in accordance with literature [99]. Furthermore, it was shown in Chapter 3 that the pulse wave propagation model has the potential to support decision-making in AVF surgery planning by predicting postoperative flow.

\subsection{Model personalization}

To adapt the model to patient-specific conditions, the model parameters and boundary conditions should be personalized. To deal with sparsity and uncertainty in patient datasets, a sensitivity analysis was performed to identify input parameters for which more accurate measurements would reduce output uncertainty (parameter prioritization) or that might be fixed and based on literature (parameter fixing). The pulse wave propagation model developed is nonlinear and non-monotone relations between input parameters and output are expected. Moreover, a large number of input parameters are involved, which increases the probability of interactions between parameters as some parameters are mathematically related within the model (non-additive model). Therefore, a local sensitivity analysis taking the local derivative of the output with respect to a single input parameter, is insufficient 
and a global sensitivity analysis is required. Therefore, a global variance-based sensitivity analysis using Monte Carlo simulations was applied that apportioned the output variance to input parameters and is considered to be the best current available practice with respect to parameter prioritization and fixing $[68,69,75]$.

This variance-based sensitivity analysis was used in Chapter $\mathbf{4}$ for factor prioritization. It was found that the results of the sensitivity analysis depended on the output of interest being either the mean brachial artery flow or the distal systolic pressure. In predictive surgery, it is thus necessary to clearly define the output of interest prior to model personalization. With respect to the mean brachial flow, it was observed that the input parameters associated with the smallest diameters in the inflow and outflow tract of the VA conduit should be measured as accurate as possible. These input parameters are also in clinics associated with flow enhancement and thus determined during the preoperative vessel assessment. In addition, the mean aortic flow, the peripheral resistance and compliance of the radial artery, the aortic peripheral resistance, the intravenous pressure and the distributed flow were identified as parameters that should be more accurately estimated to improve the precision of the model predictions. Clinically, the surgical strategy is mainly based on vessel diameters, but it might be worthwhile to consider these other influential parameters during the preoperative work-up of vascular access creation.

To identify input parameters that could be set to a generic value, a second variance-based global sensitivity analysis was performed in Chapter 5. It showed that a significant number of input parameters (47 out of 73) could be fixed within their population spread. Furthermore, we were able to determine generic values for 37 of the 47 fixable input parameters. A significant number of input parameters can thus be based on literature and, as a result, no additional measurements are needed. This would significantly simplify the personalization and thus increase the clinical applicability of the model.

\subsection{Model corroboration}

In Chapter 6, our pulse wave propagation was validated against a one-to-one silicone model of the aorta, arm arteries and veins incorporated into a mock loop [76, 77]. Silicone models were previously also used in other validation studies [63, 126, 127, 130], however, to our knowledge, it was the first time that the uncertainty of the simulations due to input parameters uncertainties was considered. Moreover, the pulse wave propagation model was not yet validated for predicting pressure and flow waveforms after inclusion of veins and the anastomosis after AVF creation. The experimental setup used allowed for model corroboration, because it was able to mimic flow enhancement after AVF creation 
and because the experimental pressure and flow waveforms were resembling waveforms observed in clinical literature [99, 131].

It was shown that the pulse wave propagation was able to capture the main features of the experimental pressure and flow waveforms both before and after AVF creation. More specifically, the mean pressures and flows were similar in experiment and simulation, whereas, experimentally, the waveforms were more attenuated which most likely results from viscoelasticity of the silicone tubes $[63,130]$.

The final step was to examine the applicability of the pulse wave propagation model to support decision-making in AVF surgery planning (Chapter 7). For this clinical corroboration the focus was on brachial artery flow. Insights obtained from the sensitivity analysis were used to create and personalize patient-specific wave propagation models for 25 ESRD patients awaiting their primary AVF creation. Input parameters were obtained from measurements and literature. Preoperatively, three AVF configurations (RCAVF, BCAVF and BBAVF) were simulated for each patient and the predicted postoperative flows were used to select the most suitable site for AVF creation. It was found that the model was capable to predict the correct postoperative flows at one week in $70 \%$ of all cases. When comparing the choice of the model with the AVF created by an experienced surgeon, the model and the surgeon fully coincided in $60 \%$, whereas differentiation between upper and lower arm AVF configuration coincided in $76 \%$ of the patients. In three patients the model allowed for a lower arm AVF, whereas the surgeon decided to create an upper arm AVF. With the additional information from the model, the surgeon might have had decided to preserve proximal vessels for future VA procedures by creating a lower arm AVF. In addition, the model predicted a too low access flow in one patient who received a lower arm AVF which failed because of immediate thrombosis. In this perspective, computational modeling can be considered as a valuable tool in the preoperative workup, in addition to the currently performed diameter measurements, although a clinical randomized trial is required to determine the exact additional value of the model.

\subsection{Future perspectives}

\section{Computational model}

Although the computational model derived correctly describes the relevant hemodynamic changes directly after AVF creation, some model improvements can be identified. The experimental corroboration showed that the extra pressure drop resulting from complex geometries like stenosis, anastomosis, and kinking or twisting vessels, should be captured 
in the model to adequately predict mean brachial flow. When a lumped parameter representation of these pressure drops is available, for example in the form of a pressure-flow relationship, these complex geometries can easily be incorporated in the pulse wave propagation model. For a stenosis and anastomosis such descriptions are already available in literature $[25,50,51,52,55]$. However, the description for the anastomosis stems from a T-junction. The pressure-flow relation of a physiological anastomosis might be different. A more physiological lumped model of the anastomosis can be obtained by analyzing the anastomosis geometry in ESRD patients and defining, based on this analysis, parameterized three-dimensional computational models. Computational fluid dynamics can be used to simulate the pressure drop over these anastomotic geometries as function of the Reynolds and Strouhal number and as function of geometrical properties like the diameter ratio of the proximal artery and proximal vein. The pressure-flow relations of other complex geometries can also be assessed by following this approach.

Besides, it is known from literature that the vasculature and heart are able to adapt to sustained alterations in hemodynamic loading. Previously, this was extensively studied by means of computational models $[154,155,156,157,158]$. The pulse wave propagation model can be further improved by incorporating vascular adaptation (e.g. flow-mediated dilatation, regulation of peripheral bed), vascular remodeling (maturation) and remodeling of the heart, especially when the long-term outcome of AVF creation is subject of study. The downside of these model improvements is the increase in input parameters and thus more complex model personalization.

\section{Model personalization}

To optimize model personalization efforts to improve estimation of the influential model parameters might be worthwhile. In the model diameters of the arm vasculature, and especially the smallest diameters, were identified as influential for the mean brachial flow. During the preoperative work-up, these diameters are obtained from duplex ultrasound measurements performed in clinical routine. As the diameters were measured at discrete locations, inter- and extrapolation was used to obtain intermediate diameters for the model. The smallest diameter and kinking or twisting vessels might have been missed during the ultrasound examination. As an alternative, magnetic resonance angiography could be used, thus obtaining an overview of the complete vessel topology [152]. However, magnetic resonance angiography has a lower resolution than ultrasound. Therefore for future studies, to fully use the advantages of both modalities, it would be beneficial to develop a modality in which diameters measured with ultrasound can be mapped onto 
magnetic resonance images.

Since the peripheral impedance of the peripheral beds in the hand and aorta were also identified as influential for the mean flow, future efforts should be put into obtaining better estimates of these model parameters. The impedance of the peripheral beds might be obtained by the method proposed by Beulen et al. [77, 159, 160] that assesses pressure and flow waveforms simultaneously in a perpendicular ultrasound measurement.

For model input parameters that are important but difficult to assess, it might be considered to apply data fitting or data assimilation techniques [45, 161, 162]. Future research should investigate which of these techniques can be applied to our pulse wave propagation model as the large number of input parameters in our model, thus the chance that more than one combination of input parameters results in good fits, complicates direct application. Another possibility might be to determine this influential, but difficult accessible, parameters, by using adaptation laws [163].

\section{Model corroboration}

Since the Doppler ultrasound flow is clinically used and the surgeon is used to deal with its uncertainty, a model that predicts postoperative flows that coincide with these ultrasound measurements might already be useful. However, to further optimize the clinical model corroboration, efforts to obtain a more accurate estimate of the postoperative flow, which serves as reference for flow validation, might be useful. First, inaccuracy in Doppler flows results from assumptions in the estimation of the blood velocity over the crosssectional area which differ between ultrasound devices and between hospitals. In our hospital, the mean blood velocity is obtained by time-averaging the outer envelope of the Doppler spectrum and assuming a flat velocity profile. However, postoperatively, the exact velocity profile is not known. In case of fully developed flow, the velocity profile is likely parabolic [84]. But, the high mean Reynolds numbers resulting in large entrance lengths and transitional flows advocate for more flat profiles. Second, the determination of the cross-sectional area by means of B-mode ultrasound further increases the inaccuracy on the flow measurements. Therefore, future efforts should be made to obtain better estimates of the immediate postoperative flow. This can be achieved by either a velocity correction method in case of fully developed flow [164, 165] or by using new ultrasound techniques that measure the complete velocity profile over the cross-sectional area [159, 160].

However, despite the inaccuracies in flow predictions and flow measurements, the model 
was already able to differentiate between an upper and lower arm AVF in $76 \%$ of all patients. Therefore, it might already be possible to use the model in a randomized clinical trial in which the model is used as additional tool for decision-making in one group and conventional decision-making is performed in the other.

\subsection{Concluding remarks}

In this thesis, a pulse wave propagation model was developed that was able to simulate the hemodynamics after AVF surgery and was able to deal with a large variety in inputs and imperfect patient datasets. An experimental setup was made that was able to mimic the flow enhancement and hemodynamics observed after AVF creation and thus allowed for model corroboration. Experimental corroboration showed that the physical phenomena are well captured and the algorithm was implemented properly.

Prior to clinical corroboration, a sensitivity analysis was successfully performed and insight was gained into how the model should be made patient-specifically. These insights were used in the personalization of pulse wave propagation models of 25 ESRD patients awaiting their first vascular access creation. It was observed that the predicted postoperative flows corresponded to the measured flows one week after surgery in $70 \%$ of all cases. In addition, it was shown that the model can support clinical decision-making in AVF surgery planning.

The advantage of the pulse wave propagation model is that it combines multiple influencing factors (e.g. vessel geometry, vessel mechanics, resistance over the anastomosis) [99] by means of physical laws. It is capable of capturing clinical knowledge in a quantitative estimate for postoperative flow. Nevertheless, the mathematical relations used to describe physical laws can be more or less complex depending on assumptions made during model development. Simplification of the mathematical relations reduces the computational time and the number of model parameters that are required for model personalized, which both eases clinical application. However, at the same time the model is also a more simplified representation of reality (model uncertainty) resulting in less accurate predictions. Since both model parameter uncertainty and model uncertainty affect the uncertainty in model predictions, a balance need to be found between model complexity and the uncertainty in model output due to input uncertainties. A proper balance results in the most accurate flow predictions but is difficult to find. Fortunately, the model can already be useful as additional tool in clinical decision-making when decision-making with the model is better than without the model. To determine this, a randomized trial is now advised.

Although it will be impossible to obtain a predictive value of the model of $100 \%$ due to 
limitations in the modeling and input measurements, an extensive collaboration between a surgeon and (qualified medical) engineer might improve the predictions and the model's applicability. Clinical insights of the surgeon in, for example, the maturation process are useful to further improve the model. In addition, improvements of the clinical measurement protocols can be achieved by a good collaboration between surgeon and medical engineer, since the surgeon has the required clinical knowledge while the medical engineer has more expertise in the physical principles of measurements and the statistical analysis of results. In addition, the engineer is able to translate the model results to improvements in measurement protocols. Good collaboration should finally result in improved patient care and reduced health care costs. 


\section{References}

[1] AC Guyton and JE Hall. Textbook of medical physiology. W.B. Saunders Company, 1996.

[2] Registration of renal replacements, www.renine.nl, the netherlands.

[3] N Lameire, K Jager, W Van Biesen, D de Bacquer, and R Vanholder. Chronic kidney disease: A European perspective. Kidney Int, S99:S30-S38, 2005.

[4] A Grassman, S Gioberge, S Moeller, and G Brown. ESRD patients in 2004: Global overview of patients number, treatment modalities and associated trends. Nephrol Dial Transplant, 20:2587-2593, 2005.

[5] AM El Nahas and RA Hamer. The burden of chronic kidney disease. British Medical Journal, 332:563-564, 2006.

[6] M Allon and ML Robbin. Increasing arteriovenous fistulas in hemodialysis patients: Problems and solutions. Kidney Int, 62:1109-1124, 2002.

[7] JHM Tordoir and V Mickley. European guidelines for vascular access: Clinical algorithms on vascular access for hemodialysis. EDTNA ERCA J, 29:131-136, 2003.

[8] A Bakran, V Mickley, and J Passlick-Deetjen. Management of the renal patient: Clinical algorithms on vascular access for haemodialysis. Pabst Science Publishers, D-49525 Lengerich, Deutschland, 2003.

[9] Vascular access work group. I: NKF-K/DOQI clinical practice guidelines for vascular access: update 2006. Am J Kidney Dis, 48(Suppl 1):S176-S276, 2006.

[10] JHM Tordoir, P Rooyens, R Dammers, FM van der Sande, M de Haan, and TI Yo. Prospective evaluation of failure modes in autogenous radiocephalic wrist access for hemodialysis. Nephrol Dial Transplant 2003; 18:, .18:378-383, 2003.

[11] R Dammers. Non-invasive Assessment of Arterial Remodeling in Arteriovenous Fistulas for Hemodialysis. PhD thesis, University of Maastricht, The Netherlands, 2003. 
[12] JHM Tordoir, R Dammers, and FM van der Sande. Upper extremity ischemia and hemodialysis vascular access. Eur J Vasc Endovasc Surg, 27:1-5, 2004.

[13] E Wijnen, XHA Keuter, RN Planken, FM van der Sande, Tordoir JHM, KM Leunissen, and JP Kooman. The relation between vascular access flow and different types of vascular access with systemic hemodynamics in hemodialysis patients. Artificial Organs, 29(12):960-964, 2005.

[14] ML Robbin, NE Chamberlain, ME Lockhart, MH Gallichio, CJ Young, MH Deierhoi, and M Allon. Hemodialysis arteriovenous fistula maturity: US evaluation. Radiology, 225:5964, 2002.

[15] GA Beathard, P Arnold, J Jackson, and T Litchfield. Aggressive treatment of early fistula failure. Kidney International, 64:1487Ü1494, 2003.

[16] BS Dixons. Why don't fistulas mature? Kidney Int, 70:1413-1422, 2006.

[17] L Turmel-Rodrigues, J Pengloan, S Baudin, D Testou, M Abaza, G Dahdah, A Mouton, and D Blanchard. Treatment of stenosis and thrombosis in haemodialysis fistulas and grafts by interventional radiology. Nephrol Dial Transplant, 15(12):2029-36, 2000.

[18] T Lee, A Ullah, M Allon, P Paul Succop, M El-Khatib, R Munda, and P Roy-Chaudhury. Decreased cumulative access survival in arteriovenous fistulas requiring interventions to promote maturation. Clin J Am Soc Nephrol, 6(3):575-581, 2011.

[19] HI Feldman, S Kobrin, and A Wasserstein. Hemodialysis vascular access morbidity. J Am Soc Nephrol, 7:523-535, 1996.

[20] M Malovrh. Native arteriovenous fistula: preoperative evaluation. Am J Kidney Dis, 39:1218-1225, 2002.

[21] V Wong, R Ward, J Taylor, S Selvakumar, TV How, and A Bakran. Factors associated with early failure of arteriovenous fistulae for haemodialysis access. Eur J Vasc Endovasc Surg, 12:207-213, 1996

[22] J van der Linden, TW Lameris, AH van den Meiracker, AAEA de Smet, PJ Blankestijn, and MA van den Dorpel. Forearm venous distensibility predicts successful arteriovenous fistula. Am J Kidney Dis, 47(6):1013-1019, 2006.

[23] YO Kim, YJ Choi, Kim JI, Kim YS, BS Kim, CW Park, HC Song, SA Yoon, YS Chang, and BK Bang. The impact of intima-media thickness of radial artery on early failure of radiocephalic arteriovenous fistula in hemodialysis patients. J Korean Med Sci, 21:284-289, 2006.

[24] YM Ku, YS Kim, YS Chang, and BK Bang. Ultrasonographic measurement of intima-media thickness of radial artery in pre-dialysis uraemic patients: Comparison with histological examination. Nephrol Dial Transplant, 21:715Ü720, 2006.

[25] N Stergiopulos, DF Young, and TR Rogge. Computer simulation of arterial flow with applications to arterial and aortic stenoses. J Biomech, 25(12):1477-1488, 1992. 
[26] DF Young and FY Tsai. Flow characteristics in models of arterial stenoses. i. steady flow. $J$ Biomech, 6:395Ú410, 1973.

[27] DF Young and FY Tsai. Flow characteristics in models of arterial stenoses. ii. unsteady flow. J Biomech, 6:547Ú59, 1973.

[28] K. Van Canneyt, T. Pourchez, S. Eloot, C. Guillame, A. Bonnet, P. Segers, and P. Verdonck. Hemodynamic impact of anastomosis size and angle in side-to-end arteriovenous fistulae: a computer analysis. Journal of Vascular Access, 11:52-58, 2010.

[29] Y Pousset, P Lermusiaux, G Berton, JM le Gouez, and R Leroy. Numerical model study of flow dynamics through an end-to-side anastomosis: Choice of anastomosis angle and prosthesis diameter. Ann Vasc Surg, 20(6):773-779, 2006.

[30] K Konner. The anastomosis of the arteriovenous fistula-common errors and their avoidance. Nephrol Dial Transplant, 17:376-379, 2002.

[31] M Malovrh. Update on preoperative assessment. EVC textbook: Vascular Access; Editors: J. Tordoir, -, Edizioni Minerva Medica, Book Chapter 2, 2009.

[32] D Shemesh, I Goldin, D Berelowitz, I Zaghal, C Zigelman, and O Olsha. Blood flow volume changes in the maturing arteriovenous fistula for hemodialysis. Ultrasound in Med Biol, 33(5):727-733, 2007.

[33] SJ Sherwin, L Formaggia, J Peiró, and V Franke. Computational modelling of 1D blood flow with variable mechanical properties and its application to simulation of wave propagation in the human arterial system. Int J Numer Meth Fluids, 43:673-70, 2003.

[34] MS Olufsen, A Nadim, and LA Lipsitz. Dynamics of cerebral blood flow regulation explained by using a lumped parameter model. Am J Physiol, 282:611-622, 2002.

[35] F Liang and H Liu. A closed-loop lumped parameter computational model for human cardiovascular system. JSME International Journal Series C, 48(4), 2005.

[36] N Westerhof, F Bosman, CJ de Vries, and A Noordergraaf. Analog studies of the human systemic arterial tree. J Biomech, 2:121-143, 1969.

[37] CA Taylor, TJR Hughes, and CK Zarins. Finite element modeling of blood flow in arteries. Comp Meth Appl Mech Eng, 158:155-196, 1998.

[38] Z Kharboutly, M Fenech, JM Treutenaere, I Claude, and C Legallais. Investigations into the relationship between hemodynamics and vascular alterations in an established arteriovenous fistula. Med Eng Phys, 29:999-1007, 2007.

[39] JF Wenk. Numerical modeling of stress in stenotic arteries with microcalcifications: A parameter sensitivity study. J Biomech Eng, 133(1):014503-1-014503-6, 2011.

[40] BJBM Wolters, M Emmer, MCM Rutten, GWK Schurink, and FN van de Vosse. Assessment of endoleak significance after endovascular repair of abdominal aortic aneurysms: A lumped parameter model. Med Eng Phys, 29:1106-1118, 2007. 
[41] F Migliavacca, L Petrini, M Colombo, F Auricchio, and R Pietrabissa. Mechanical behavior of coronary stents investigated through the finite element method. J Biomech, 2002:803-811, 35 .

[42] F Migliavacca, F Gervasco, M Prosi, P Zunino, S Minisini, and et al. Expansion and drug elution model of a coronary stent. Comp Meth Biomech Eng, 10:63-73, 2007.

[43] T Alderliesten, MK Konings, and WJ Niessen. Simulation of minimally invasive vascular interventions for training purposes. Computer aided surgery, 9:3-15, 2004.

[44] TY Euliano, D Caton, W van Meurs, and ML Good. Modeling obstetric cardiovascular physiology on a full-scale patient simulator. Journal of Clinical Monitoring and Computing, 13(5):293-297, 1997.

[45] CAD Leguy, EMH Bosboom, H Gelderblom, APG Hoeks, and FN van de Vosse. Estimation of distributed arterial mechanical properties using a wave propagation model in a reverse way. Med Eng Phys, 32(9):957-968, 2010.

[46] N Stergiopulos, JJ Meister, and N Westerhof. Evaluation of methods for estimation of total arterial compliance. Am J Physiol, 268:H1540-H1548, 1995.

[47] N Stergiopulos, BE Westerhof, and N Westerhof. Total arterial inertance as the fourth element of the windkessel model. Am J Physiol, 276(1 Pt 2):H81-88, 1999.

[48] L Speelman, EMH Bosboom, GWH Schurink, Hellenthal FAMVI, J Buth, M Breeuwer, MJ Jacobs, and FN van de Vosse. Patient-specific AAA wall stress analysis: 99-percentile versus peak stress. European Journal of Vascular and Endovascular Surgery, 36(6):668-676, 2008.

[49] NHJ Pijls, B van Gelder, P van der Voort, KH Peels, FALE Bracke, JJRM Bonnier, and MIH El Gamal. Fractional flow reserve: a useful index to evaluate the influence of an epicardial coronary stenosis on myocardial blood flow. Circulation, 92:3183-3193, 1995.

[50] E Marchandise, M Willemet, and V Lacroix. A numerical hemodynamic tool for predictive vascular surgery. Med Eng Phys, 31:131-144, 2002.

[51] BN Steele, J Wan, JP Ku, TJR Hughes, and CA Taylor. In vivo validation of a onedimensional finite-element method for predicting blood flow in cardiovascular bypass grafts. IEEE Transactions Biomed Eng, 50 (6):649-55, 2003.

[52] J Wan, BN Steele, CA Taylor, et al. A one-dimensional finite element method for simulationbased medical planning for cardiovascular disease. .Comp Meth Biomech Biomed Eng, 5(3):195-206, 2002.

[53] B Ene-Iordache, L Mosconi, G Remuzzi, and A Remuzzi. Computational fluid dynamics of a vascular access case for hemodialysis. J Biomech Eng, 123:284-292, 2001.

[54] K van Canneyt, T Pourchez, S Eloot, C Guillaume, A Bonnet, P Segers, and P Verdonck. Hemodynamic impact of anastomosis size and angle in side-to-end arteriovenous fistulae: A computer analysis. J Vasc Access, 11(1):52-8, 2010. 
[55] FN van de Vosse and N Stergiopulos. Pulse wave propagation in the arterial tree. Аnпu Rev Fluid Mech, 43:467-499, 2011.

[56] O Frank. Die grundform des arterielen pulses erste abhandlung: Mathematische analyse. $Z$ Biol, 37:483-526, 1899.

[57] N Westerhof, G Elzinga, and P Sipkema. An artificial arterial system for pumping hearts. $J$ Appl Phys, 31(5):776-781, 1971.

[58] R Pietrabissa, S Mantero, T Marotta, and L Menicanti. A lumped parameter model to evaluate the fluid dynamics of different coronary bypasses. Med Eng Phys, 18(6):477-484, 1996

[59] T Hughes and J Lubliner. On the one-dimensional theory of blood flow in the large vessels. Math Biosc, 18:161-170, 1973.

[60] K Azer and CS Peskin. A one-dimensional model of blood flow in arteries with friction and convection based on the Womersley velocity profile. Cardiovasc Eng, 7:51-73, 2007.

[61] D Bessems, MCM Rutten, and FN van de Vosse. A wave propagation model of blood flow in large arteries using an approximated velocity profile function. J Fluid Mech, 580:145-68, 2007.

[62] MS Olufsen, CS Peskin, WY Kim, EM Pedersen, A Nadim, and J Larsen. Numerical simulation and experimental validation of blood flow in arteries with structured-tree outflow conditions. Ann Biomed Eng, 28:1281-1299, 2000.

[63] D Bessems, CG Giannopapa, MCM Rutten, and van de Vosse FN. Experimental validation of a time-domain-based wave propagation model of blood flow in viscoelastic vessels. $J$ Biomech, 41:284-91, 2008.

[64] GN Jager. Electrical model of the human systemic arterial tree. $\mathrm{PhD}$ thesis, University of Utrecht, The Netherlands, 1965.

[65] P Reymond, F Merenda, F Perren, D Rüfenacht, and N Stergiopulos. Validation of a onedimensional model of the systemic arterial tree. Am J Physiol Heart Circ Physiol, 297:208222, 2009.

[66] SR Hanna. Air quality model evaluation and uncertainty. Journal of the Air Pollution Control Association, 38:406-442, 1988.

[67] Guidance on the development, evaluation, and application of environmental models, United States environmental protection agency, http://www.epa.gov/crem/library/cred_guidance_0309.pdf.

[68] A Saltelli, S Tarantola, F Campolongo, and M Ratto. Sensitivity analysis in practice. A guide to assessing scientific models. John Wiley and Sons Ltd, 2004.

[69] A Saltelli, M Ratto, and T Anders. Global sensitivity analysis, The primer. John Wiley and Sons Ltd, 2008. 
[70] LM Ellwein, T Hien, T Tran, C Zapata, V Novak, and MS Olufsen. Sensitivity analysis and model assessment: Mathematical models for arterial blood flow and blood pressure. Cardiovasc Eng, 8:94Ü108, 2008.

[71] D Xiu and SJ Sherwin. Parametric uncertainty analysis of pulse wave propagation in a model of a human arterial network. J Comp Phys, 226:1385-1407, 2007.

[72] CAD Leguy, EMH Bosboom, ASZ Belloum, APG Hoeks, and FN van de Vosse. Global sensitivity analysis of a wave propagation model in arm arteries. Med Eng Phys, In press, 2011.

[73] L Grinberg, E Cheever, T Anor, JR Madsen, and GE Karniadakis. Modeling blood flow circulation in intracranial arterial networks: a comparative 3D/1D simulation study. Ann Biomed Eng, 39:297-309, 2011.

[74] A Hoare, DG Regan, and DP Wilson. Sampling and sensitivity analysis tools (SaSAT) for computational modelling. Theor Bio Med Mod, 5(4):1-18, 2008.

[75] A Saltelli, P Annoni, I Azzini, F Campolongo, M Ratto, and S Tarantola. Variance based sensitivity analysis of model output. design and estimator for the total sensitivity index. Comp Phys Comm, 181:259-270, 2010.

[76] $M$ in 't Veer, MCF Geven, MCM Rutten, A van der Horst, WH Aarnoudse, NHJ Pijls, and FN van de Vosse. Continuous infusion thermodilution for assessment of coronary flow: theoretical background and in vitro validation. Med Eng Phys, 31(6):688-694, 2009.

[77] BWAMM Beulen, N Bijnens, GG Koutsouridis, PJ Brands, MCM Rutten, and FN van de Vosse. Toward noninvasive blood pressure assessment in arteries by using ultrasound. $\mathrm{Ul}$ trasound in Med Biol, 37(5):788-797, 2011.

[78] N Stergiopulos, JJ Meister, and N Westerhof. Simple and accurate way for estimating total and segmental arterial compliance: the pulse pressure method. Ann Biomed Eng, 22:392397, 1994.

[79] R Fogliardi, R Burattini, SG Shroff, and KB Campbell. Fit to diastolic arterial pressure by third-order lumped model yields unreliable estimates of arterial compliances. Med Eng Phys, 18:225-233, 1996

[80] LR Hellevik, P Segers, N Stergiopulos, P Irgens, P Verdonck, CR Thompson, K Lo, RT Miyagishima, and OA Smiseth. Mechanism of pulmonary venous pressure and flow waves. Heart Vessels, 14:67-71, 1999.

[81] P Segers, N Stergiopulos, JJ Schreuder, BE Westerhof, BE Westerhof, and N Westerhof. Left ventricular wall stress normalization in chronic pressure-overloaded heart: a mathematical model study. Am J Physiol Heart Circ Physiol, 279:1120-1127, 2000.

[82] A Noordergraaf, PD Verdouw, and HBK Boom. The use of an analog computer in a circulation model. Prog Cardiovasc Dis, 5:419-439, 1963.

[83] MS Olufsen and A Nadim. On deriving lumped models for blood flow and pressure in the systemic arteries. Math Biosc Eng, 1:61-80, 2004. 
[84] JR Womersley. Method for the calculation of velocity, rate of flow and viscous drag in arteries when the pressure gradient is known. J Physiol, 127:553-563, 1955.

[85] NW McLachlan. Bessel functions for engineers. Oxford University Press, London, The United Kingdom, 1961.

[86] W Huberts, EMH Bosboom, and FN Van Devan de Vosse. A lumped model for blood flow and pressure in the systemic arteries based on an approximated velocity profile function. Math Biosc Eng, 6:27-40, 2009.

[87] P Segers, N Stergiopulos, P Verdonck, and R Verhoeven. Assessment of distributed arterial pulse wave propagation models. Med Biol Eng Comput, 35:729-736, 1997.

[88] M Haslam and M Zamir. Pulsatile flows in tubes of elliptical cross sections. Ann Biomed Eng, 26:780-87, 1998

[89] SA Jones, S Jin, A Kantak, DA Bell, and WD Paulson. Mathematical model for pressure losses in the hemodialysis graft vascular circuit. J Biomech Eng, 127:60-6, 2005.

[90] DJ Wood, LS Reddy, and JE Funk. Modeling pipe networks dominated by junctions. $J$ Hydr Eng, 119:949-58, 2005.

[91] A Gardel. Pressure drops in flows through T-shaped fittings. Bulletin Technique de la Suisse Romande, 9:123-130, 1957.

[92] A Gardel. Pressure drops through T-shaped fittings. Bulletin Technique De La Suisse Romande, pages 143-148, 1957.

[93] RN Planken, XHA Keuter, APG Hoeks, JP Kooman, FM van der Sande, AGH Kessels, $\mathrm{T}$ Leiner, and JHM Tordoir. Diameter measurements of the forearm cephalic vein prior to vascular access creation in End-stage Renal Disease patients: Graduated pressure cuff versus tourniquet vessel dilatation. Nephr Dia Trans, 21(3):802-806, 2006.

[94] RN Planken, XHA Keuter, AGH Kessels, APG Hoeks, T Leiner, and JHM Tordoir. Forearm cephalic vein cross-sectional area changes at incremental congestion pressures: Towards a reproducible and standardized vein mapping protocol. J Vasc Surg, 44(2):353-358, 2006.

[95] DR Kaiser, K Mullen, and AJ Bank. Brachial artery elastic mechanics in patients with heart failure. Hypertension, 38:1440Ü1445, 2001.

[96] JJ Mourad, X Girerd, P Boutouyrie, S Laurent, M Safar, and G London. Increased stiffness of radial artery wall material in end-stage renal disease. Hypertension, 30:1425Ü1430, 1997.

[97] W Huberts, EMH Bosboom, RN Planken, JHM Tordoir, and FN van de Vosse. Patientspecific computational modeling to improve the clinical outcome of vascular access creation. EVC Textbook 2009: Vascular Access, pages 88-7711, 2009. ; Editors: J. Tordoir, -, Edizioni Minerva Medica, Book Chapter ISBN 10:-640-4.

[98] CD Murray. The physiological principle of minimum work: In the vascular system and the cost of blood volume. Proc Natl Acad Sci USA, 12:207-214, 1926. 
[99] DE Strandness and DS Sumner. Hemodynamics for surgeons. New York (NY), Grune and Stratton, 1975.

[100] I van Tricht, D de Wachter, JHM Tordoir, and P Verdonck. Hemodynamics and complications encountered with AVFs and grafts as vascular access for hemodialysis: A review. Ann Biomed Eng, 33(9):1142-1157, 2005.

[101] W Huberts, AS Bode, W Kroon, RN Planken, JHM Tordoir, FN van de Vosse, and EMH Bosboom. A pulse wave propagation model to support decision-making in vascular access planning in the clinic. Med Eng Phys, Online available, doi: 10.1016/j.medengphy.2011.07.015, 2011.

[102] JC Helton. Uncertainty and sensitivity analysis techniques for use in performance assessment for radioactive waste disposal. Reliability Eng Syst Safety, 42:327-367, 1993.

[103] A Saltelli. Making best use of model evaluations to compute sensitivity indices. Comp Phys Comm, 145:280-297, 2002.

[104] DC Montgomery and GC Runger. Applied statistics and probability for engineers. John Wiley and Sons Ltd, 1999.

[105] IM Sobol. Global sensitivity analysis for nonlinear mathematical models and their Monte Carlo estimates. Math Comp in Sim, 55:271-280, 2001.

[106] T Homma and A Saltelli. Importance measures in global sensitivity analysis of model output. Reliability Eng System Safety, 52 (1):1Ű17, 1996.

[107] S Li, WN McDicken, and PR Hoskins. Blood vessel diameter measurement by ultrasound. Physiol Meas, 14:291-297, 1993.

[108] F Molinari, G Zeng, and JS Suric. A state of the art review on intima-media thickness (IMT) measurement and wall segmentation techniques for carotid ultrasound. Computer Methods and Programs in Biomedicine, 100(3):201 - 221, 2010.

[109] J Lotz, C Meier, A Leppert, and M Galanski. Cardiovascular flow measurement with phasecontrast MR imaging: Basic facts and implementation. Radiographics, 22(3):651-671, 2002.

[110] DW Eeftinck Schattenkerk, JJ van Lieshout, AH van den Meiracker, KR Wesseling, S Blanc, W Wieling, GA van Montfrans, JJ Settels, KH Wesseling, and BE Westerhof. Nexfin noninvasive continuous blood pressure validated against Riva-Rocci/Korotkoff. Am J Hypertens, 22:378-383, 2009.

[111] MD McKay, RJ Beckman, and WJ Conover. A comparison of three methods for selecting values of input variables in the analysis of output from a computer code. Technometrics, 21:239-245, 1979.

[112] GEB Archer, A Saltelli, and IM Sobol. Sensitivity measures, ANOVA-like techniques and the use of bootstrap. J Statist Comput Simul, 58:99-120, 1997.

[113] C Xu and GZ Gertner. A general first-order global sensitivity analysis method. Reliability Eng Syst Safety, 93:1060-1071, 2008. 
[114] J Jacques, C Lavergne, and N Devictor. Sensitivity in the presence of model uncertainty and correlated input. Reliability Eng Syst Safety, 91:1126-1134, 2006.

[115] M Ratto, S Tarantola, and A Saltelli. Estimation of importance indicators for correlated inputs. Proc of ESREL 2001, pages 157-164, 2001.

[116] S Da Veiga, F Wahl, and F Gamboa. Local polynomial estimation for sensitivity analysis on models with correlated inputs. Technometrics, 51(4):452-463, 2009.

[117] CB Storlie and JC Helton. Multiple predictor smoothing methods for sensitivity analysis: description of techniques. Reliability Eng Syst Safety, 93:28-54, 2008.

[118] CB Storlie, LP Swiler, JC Helton, and CJ Sallaberry. Implementation and evaluation of nonparametric regression procedures for sensitivity analysis of computationally demanding models. Reliability Eng Syst Safety, 94:1735-1763, 2009.

[119] KT Fang, R Li, and A Sudjianto. Design and modeling for computer experiments. Chapman and Hall/CRC, 2006.

[120] T Ishigami and T Homma. An importance quantification technique in uncertainty analysis for computer models. In Proceedings of the ISUMA90, First International Symposium on Uncertainty Modelling and Analysis, December 3-6, University of Maryland, 1990.

[121] W Huberts, C de Jonge, WPM van der Linden, MA Inda, JHM Tordoir, FN van de Vosse, and EMH Bosboom. A sensitivity analysis to improve the measurement protocol to assess parameters for a personalized wave propagation model: application to AVF surgery. Submitted to Med Eng Phys, 2011.

[122] A Bode, A Caroli, W Huberts, N Planken, L Antiga, M Bosboom, A Remuzzi, and J Tordoir. Clinical study protocol for the ARCH project - computational modeling for improvement of outcome after vascular access creation. J Vasc Access, 31, 2011.

[123] CJ Konings, R Dammers, PL Rensma, JP Kooman, AP Hoeks, L Kornet, U Gladziwa, FM van der Sande, and KM Leunissen. Arterial wall properties in patients with renal failure. Am J Kidney Dis, 39(6):1206-1212., 2002.

[124] R Dammers, F Stifft, JHM Tordoir, APG Hameleers JMM, Hoeks, and PJEHM Kitslaar. Shear stress depends on vascular territory: comparison between common carotid and brachial artery. J Appl Physiol, 94:485-489, 2003.

[125] P Reymond, Y Bohraus, F Perren, F Lazeyras, and N Stergiopulos. Validation of a patientspecific one-dimensional model of the systemic arterial tree. Am J Physiol Heart Circ Physiol, 301(3):H1173-H1182, Sep 2011.

[126] K.S. Matthys, J. Alastrueya, J. Peiro, A.W. Khir, P. Segers, P.R. Verdonck, K.M. Parker, and S.J. Sherwin. Pulse wave propagation in a model human arterial network: Assessment of 1D numerical simulations against in vitro measurements. Journal of Biomechanics, 40:34763486, 2007.

[127] A. Swillens, L. Lanoye, J. De Backer, N. Stergiopulos, P.R. Verdonck, F. Vermassen, and S. Segers. Effect of an abdominal aortic aneurysm on wave reflection in the aorta. IEEE Transactions on biomedical engineering, 55(5):1602-1611, 2008. 
[128] CP Robert and GC Casella. Monte Carlo statistical methods. Springer, New York, 2000.

[129] K. Van Canneyt, R.N. Planken, S. Eloot, P. Segers, and P. Verdonck. Experimental study of a new method for early detection of vascular access stenoses: Pulse pressure analysis at hemodialysis needle. Artificial Organs, 34(2):113-117, 2010.

[130] J Alastruey, AW Khir, KS Matthys, P Segers, SJ Sherwin, PR Verdonck, KH Parker, and J Peiró. Pulse wave propagation in a model human arterial network: Assessment of 1D visco-elastic simulations against in vitro measurements. J Biomech, 44:2250-2258, 2011.

[131] WW Nichols and MF O'Rourke. McDonalds's blood flow in arteries : theoretical, experimental and clinical principles. Edward Arnold, London, 1998.

[132] Rashmi Raghu, Irene E Vignon-Clementel, C. Alberto Figueroa, and Charles A Taylor. Comparative study of viscoelastic arterial wall models in nonlinear one-dimensional finite element simulations of blood flow. J Biomech Eng, 133(8):081003, Aug 2011.

[133] BN Steele, D Valdez-Jasso, MA Haider, and MS Olufsen. Predicting arterial flow and pressure dynamics using a 1D fluid dynamics model with a viscoelastic wall. Siam J Appl Math, 71 (4):1123-1143, 2011.

[134] B Learoyd and M Taylor. Alterations with age in the viscoelastic properties of human arterial walls. Clinical Research, 18:278-292, 1966.

[135] T Imura, K Yamamoto, T Satoh, K Kanamori, T Mikami, and H Yasuda. In vivo viscoelastic behavior in the human aorta. Circulation Research, 66:1413-1419, 1990.

[136] Anton N Sidawy, Richard Gray, Anatole Besarab, Mitchell Henry, Enrico Ascher, Michael Silva, Arnold Miller, Larry Scher, Scott Trerotola, Roger T Gregory, Robert B Rutherford, and K. Craig Kent. Recommended standards for reports dealing with arteriovenous hemodialysis accesses. J Vasc Surg, 35(3):603-610, Mar 2002.

[137] Jan Tordoir, Bernard Canaud, Patrick Haage, Klaus Konner, Ali Basci, Denis Fouque, Jeroen Kooman, Alejandro Martin-Malo, Luciano Pedrini, Francesco Pizzarelli, James Tattersall, Marianne Vennegoor, Christoph Wanner, Piet ter Wee, and Raymond Vanholder. EBPG on vascular access. Nephrol Dial Transplant, 22 Suppl 2:ii88-i117, May 2007.

[138] Ronald L Pisoni, Charlotte J Arrington, Justin M Albert, Jean Ethier, Naoki Kimata, Mahesh Krishnan, Hugh C Rayner, Akira Saito, Jeffrey J Sands, Rajiv Saran, Brenda Gillespie, Robert A Wolfe, and Friedrich K Port. Facility hemodialysis vascular access use and mortality in countries participating in dopps: an instrumental variable analysis. Am J Kidney Dis, 53(3):475-491, Mar 2009.

[139] David A Vorp. Biomechanics of abdominal aortic aneurysm. J Biomech, 40(9):1887-1902, 2007.

[140] M. Breeuwer, S. de Putter, U. Kose, L. Speelman, K. Visser, F. Gerritsen, R. Hoogeveen, R. Krams, H. van den Bosch, J. Buth, T. Gunther, B. Wolters, E. van Dam, and F. van de Vosse. Towards patient-specific risk assessment of abdominal aortic aneurysm. Med Biol Eng Comput, 46(11):1085-1095, Nov 2008. 
[141] T. David and S. Moore. Modeling perfusion in the cerebral vasculature. Med Eng Phys, 30(10):1227-1245, Dec 2008.

[142] Juan R Cebral, Marcelo A Castro, James E Burgess, Richard S Pergolizzi, Michael J Sheridan, and Christopher M Putman. Characterization of cerebral aneurysms for assessing risk of rupture by using patient-specific computational hemodynamics models. AJNR Am J Neuroradiol, 26(10):2550-2559, 2005.

[143] Peter J Hunter, Andrew J Pullan, and Bruce H Smaill. Modeling total heart function. Annu Rev Biomed Eng, 5:147-177, 2003.

[144] Frank J H Gijsen, Jolanda J Wentzel, Attila Thury, Bram Lamers, Johan C H Schuurbiers, Patrick W Serruys, and Antonius F van der Steen. A new imaging technique to study 3D plaque and shear stress distribution in human coronary artery bifurcations in vivo. J Biomech, 40(11):2349-2357, 2007.

[145] U Krueger, J Zanow, and H Scholz. Comparison of two different arteriovenous anastomotic forms by numerical 3D simulation of blood flow. Int J Angiol, 9(4):226-231, Oct 2000.

[146] U Krueger, J Zanow, and H Scholz. Computational fluid dynamics and vascular access. Artif Organs, 26(7):571-575, Jul 2002.

[147] Wayne S Gradman and Costantine Pozrikidis. Analysis of options for mitigating hemodialysis access-related ischemic steal phenomena. Ann Vasc Surg, 18(1):59-65, Jan 2004.

[148] W Huberts, C de Jonge, WPM van der Linden, MA Inda, JHM Tordoir, FN van de Vosse, and Bosboom EMH. A sensitivity analysis to reduce the number of input parameters in a personalized pulse wave propagation model for AVF surgery. Submitted to Med Eng Phys, 2011.

[149] WD McArdle, FI Katch, and VL Katch. Exercise physiology : nutrition, energy, and human performance. Lippincott Williams and Wilkins, London, 2010.

[150] M. B. Silva, R. W. Hobson, P. J. Pappas, Z. Jamil, C. T. Araki, M. C. Goldberg, G. Gwertzman, and F. T. Padberg. A strategy for increasing use of autogenous hemodialysis access procedures: impact of preoperative noninvasive evaluation. J Vasc Surg, 27(2):302-7; discussion 307-8, Feb 1998.

[151] I. Mihmanli, K. Besirli, S. Kurugoglu, K. Atakir, S. Haider, G. Ogut, F. Numan, E. Canturk, and A. G. Sayin. Cephalic vein and hemodialysis fistula: surgeon's observation versus color Doppler ultrasonographic findings. J Ultrasound Med, 20(3):217-222, Mar 2001.

[152] RN Planken, T Leiner, and RJ Nijenhuis. Contrast-enhanced magnetic resonance angiography findings prior to hemodialysis vascular access creation: a prospective analysis. $J$ Vasc Access, 9(4):269-277, 2008.

[153] W Kroon, W Huberts, EMH Bosboom, and FN van de Vosse. An algorithm of reduced complexity for simulating vascular hemodynamics using 0D lumped and 1D wavepropagation models. Comp Math Meth Med, Submitted, 2011. 
[154] J. D. Humphrey. Vascular mechanics, mechanobiology, and remodeling. J Mech Med Biol, 9(2):243-257, 2009.

[155] J. D. Humphrey. Vascular adaptation and mechanical homeostasis at tissue, cellular, and sub-cellular levels. Cell Biochem Biophys, 50(2):53-78, 2008.

[156] Wilco Kroon, Tammo Delhaas, Peter Bovendeerd, and Theo Arts. Structure and torsion in the normal and situs inversus totalis cardiac left ventricle. ii. modeling cardiac adaptation to mechanical load. Am J Physiol Heart Circ Physiol, 295(1):H202-H210, Jul 2008.

[157] Wilco Kroon, Tammo Delhaas, Theo Arts, and Peter Bovendeerd. Computational modeling of volumetric soft tissue growth: application to the cardiac left ventricle. Biomech Model Mechanobiol, 8(4):301-309, Aug 2009.

[158] Wilco Kroon, Tammo Delhaas, Peter Bovendeerd, and Theo Arts. Computational analysis of the myocardial structure: adaptation of cardiac myofiber orientations through deformation. Med Image Anal, 13(2):346-353, Apr 2009.

[159] BWAMM Beulen, N Bijnens, MCM Rutten, Brands PJ, and FN van de Vosse. Perpendicular ultrasound velocity measurement by $2 \mathrm{D}$ cross-correlation of RF-data. validation in a straight tube. Exp Fluids, 49(5):1177-1186, 2010.

[160] BWAMM Beulen, AC Verkaik, N Bijnens, MCM Rutten, and FN van de Vosse. Perpendicular ultrasound velocity measurement by $2 \mathrm{D}$ cross-correlation of RF data: Volume flow estimation in curved vessels. Exp Fluids, 49(6):1219-1229, 2010.

[161] A Tarantola. Inverse problem theory and methods for model parameter estimation. Philadelphia : SIAM, 2005.

[162] E Kalnay. Atmospheric modeling, data assimilation and predictability. Cambridge : Cambridge University Press, 2003.

[163] Theo Arts, Tammo Delhaas, Peter Bovendeerd, Xander Verbeek, and Frits W Prinzen. Adaptation to mechanical load determines shape and properties of heart and circulation: the circadapt model. Am J Physiol Heart Circ Physiol, 288(4):H1943-H1954, Apr 2005.

[164] CAD Leguy, EMH Bosboom, Hoeks APG, and van de Vosse FN. Model-based assessment of dynamic arterial blood volume flow from ultrasound measurements. Med Biol Eng Comput, 47(6):641-648, 2009.

[165] CAD Leguy, EMH Bosboom, APG Hoeks, and FN van de Vosse. Assessment of blood volume flow in slightly curved arteries from a single velocity profile. J Biomech, 42(11):1664$1672,2009$. 


\section{Summary}


A vascular access serves as a lifeline for an end-stage renal disease (ESRD) patient since it facilitates connection of the patient to an artificial kidney for hemodialysis treatment. For efficient treatment the blood flow must be as high as $600 \mathrm{ml} / \mathrm{min}$ and because of repeated cannulation, the vascular access should be easily accessible for repeated cannulation over time. The preferred vascular access is an arteriovenous fistula (AVF) in the arm, which is a surgically created connection between artery and vein, resulting in an significant flow increase (five- to thirtyfold) and vessel dilatation and remodeling. However, AVFs are compromised by early failure due to thrombosis and non-maturation (up to 50\%), particularly in lower arm AVFs, and by long-term complications like cardiac failure and hand ischemia (up to 20\%) in upper arm AVFs. As a result, additional interventions are required to keep a vascular access suitable for hemodialysis.

The vascular surgeon tries to minimize the complications associated with an AVF by choosing the optimal AVF configuration for a specific patient. But, optimizing the AVF configuration is a difficult task, since postoperative flow enhancement differs between patients, and is affected by multiple factors (e.g. geometry and topology of the vascular system, vessel mechanics and peripheral beds). In clinical practice, the surgeon aims to collect as much information as possible regarding these influential factors before he selects the optimal site for AVF creation. For this, an extensive preoperative vessel assessment is performed. Nevertheless, complications as non-maturation, cardiac failure and hand ischemia remain predominant.

Early thrombosis and non-maturation are associated with a too low flow enhancement after AVF creation, whereas cardiac failure and hand ischemia are associated with a too high postoperative flow. A low distal systolic finger pressure is also indicative for hand ischemia. Hence, a tool that preoperatively predicts the postoperative hemodynamics can support in the selection of the optimal AVF configuration. A computational model fed by patient-specific data can quantitatively integrate the influential factors by means of physical laws and can estimate the postoperative flow and distal systolic pressure. Moreover, the effect of different AVF configurations can be examined with a computational model prior to AVF surgery. Therefore, this thesis was aimed at developing a patient-specific computational model that is able to predict the postoperative hemodynamics and examining its applicability to support decision-making in AVF surgery planning.

In Chapter 2 and 3 a 1D pulse wave propagation model was developed that is able to simulate the changes in hemodynamics after AVF creation. Pressure and flows are calculated at specific locations distributed throughout the arterial and venous tree. The proposed pulse wave propagation model allows for the incorporation of nonlinear pressureflow relations for the anastomosis, i.e. the connection between artery and vein, without 
the need for additional coupling equations as coupling is implicitly captured in the model assembly.

To adapt the computational model to patient-specific conditions, model input parameters had to be personalized. In the strategy to deal with sparsity and uncertainty in patient datasets, a global variance-based sensitivity analysis using Monte Carlo simulations was applied. In this way input parameters were identified for which more accurate measurements would reduce uncertainty in the predictions (parameter prioritization, Chapter 4), while other input parameters could be fixed and based on literature (parameter fixing, Chapter 5). The results of this sensitivity analysis importantly simplified model personalization as it was found that for only 16 out of 73 input parameters it was rewarding to improve measurement accuracy, whereas 47 out of 73 parameters could be based on literature.

In Chapter 6 the pulse wave propagation model was successfully corroborated with an experimental setup, mimicking pressure and flow changes following AVF creation. It was shown that the model was able to correctly simulate the physical phenomena after AVF creation, although the simulated pressure and flow waveforms were slightly less attenuated than the measured waveforms likely resulting from viscoelastic properties in the experimental setup.

The insights obtained in the sensitivity analysis and the experimental corroboration, were used in Chapter 7 to examine the clinical applicability of the pulse wave propagation model. For 25 ESRD patients, simulation results were compared to clinical outcome. The pulse wave propagation model was able to predict the brachial artery flow, measured with Doppler ultrasound at one week, in $70 \%$ of 25 ESRD patients. Moreover, in $76 \%$ of the patients, the pulse wave propagation model suggested the same AVF location, i.e. upper or lower arm AVF, as a very experienced surgeon. The patient-specific pulse wave propagation model might thus be beneficial for decision-making in AVF surgery planning. Future research should demonstrate the additional value of the pulse wave propagation model. 


\section{Samenvatting}


Een vaattoegang is voor patiënten die lijden aan nierfalen letterlijk hun levenslijn. De vaattoegang wordt namelijk tijdens hemodialyse gebruikt om de patiënt aan te sluiten op een kunstnier. Voor een efficiënte hemodialyse moet de bloedstroom door de vaattoegang ongeveer $600 \mathrm{ml}$ per minuut zijn. Daarnaast moet de vaattoegang geschikt zijn om gemakkelijk herhaaldelijk aan te prikken. De eerste keuze voor een vaattoegang is een arterioveneuze fistel (AVF). Dit is een chirurgisch gemaakte verbinding tussen een arterie en vene, meestal in de arm. Doordat deze verbinding wordt gemaakt neemt de bloedstroom in de betrokken bloedvaten sterk toe (vijf tot dertig keer), vindt er verwijding van de vaten plaats en remodelleert de vaatwand. Een groot probleem bij AVFs is echter dat AVFs, en in het bijzonder onderarmfistels, vaak dicht gaan zitten (thrombose) of onvoldoende matureren in de eerste zes weken na aanleg ( in 20 tot $50 \%$ van alle onderarmfistels). Bovenarmfistels geven juist vooral problemen op de lange termijn, zoals hartfalen of slechte doorbloeding (ischemie) van de hand (bij 20\% van de bovenarmfistels). Als gevolg van deze complicaties zijn er extra interventies nodig om de vaattoegang geschikt te maken en/of te houden voor hemodialyse. Voor de operatie kiest de vaatchirurg voor elke individuele patiënt de optimale locatie voor de aanleg van de AVF. Daarbij moet hij rekening houden met veel verschillende factoren die de bloedstroomtoename als gevolg van aanleg van de AVF beïnvloeden, zoals de geometrie en topologie van de vaten en de mechanische en hemodynamische eigenschappen van de vaten en het perifere vaatbed. In de huidige klinische praktijk, probeert de chirurg zoveel mogelijk informatie te verzamelen met betrekking tot deze factoren, alvorens hij bepaalt waar de AVF het beste gemaakt kan worden. Hiervoor wordt er een uitgebreid onderzoek gedaan naar het vaatbed met behulp van palpatie en ultrageluid. Echter, ondanks het preoperatieve vaatonderzoek komen complicaties, zoals non-maturatie, hartfalen en hand-ischemie nog steeds veel voor. Een te lage bloedstroom na aanleg van de de AVF gaat gepaard met directe thrombose en non-maturatie van de AVF, terwijl een hoge bloedstroom gepaard gaat met hand-ischemie en hartfalen. Ook een lage systolische vingerdruk is indicatief voor hand-ischemie. Een methode waarmee de postoperatieve bloedstroom en systolische vingerdruk voor de operatie voorspeld kunnen worden, zou de chirurg kunnen ondersteunen bij het selecteren van de optimale locatie voor de AVF. Met een computermodel dat is gebaseerd op fysische wetten en waarvan de parameters patiëntspecifiek gemaakt zijn kunnen de factoren, die van invloed zijn op de postoperatieve bloedstroom en vingerdruk, en hun onderlinge samenhang bestudeerd worden. Een dergelijk computermodel kan daarnaast ook ingezet worden om voor de operatie de effecten te vergelijken van aanleg van AVFs op verschillende locaties. Het doel van dit proefschrift is derhalve om een patiëntspecifiek computermodel te ontwikkelen dat in staat is om de postoperatieve hemodynamica te beschrij- 
ven en om te onderzoeken of een computermodel een chirurg kan ondersteunen bij het selecteren van de meeste geschikte locatie voor aanleg van de AVF. In hoofdstuk 2 en $\mathbf{3}$ van dit proefschrift wordt het $1 \mathrm{D}$ golfvoortplantingsmodel beschreven dat in staat is om de hemodynamische veranderingen na aanleg van een AVF te simuleren. Met dit model worden de bloeddruk en bloedstroom berekend op verschillende locaties in de arteriële en veneuze vaatboom. Voor het modelleren van de anastomose, de verbinding tussen arterie en vene, is er gebruik gemaakt van een niet-lineaire relatie tussen de bloeddruk en bloedstroom. Hiervoor zijn geen extra koppelingsvergelijken nodig, door op een slimme manier het stelsel vergelijkingen te assembleren. Om het computer model te personifiëren moeten de input parameters patiëntspecifiek gemaakt worden. Klinische data hebben echter vaak een grote meetonzekerheid en datasets zijn vaak incompleet. Om inzicht te verkrijgen in hoe hiermee omgegaan moet worden is er een globale gevoeligheidsanalyse uitgevoerd op het model, waarbij verschillende variantie indices zijn bepaald. Hiermee zijn de modelparameters geïdentificeerd die je het meest nauwkeurig zou moeten meten, omdat een nauwkeurige bepaling van deze parameters zal leiden tot de grootste afname van onzekerheid in de modelvoorspellingen (parameter prioritering, hoofdstuk 4). Daarnaast zijn er parameters geïdentificeerd die gebaseerd kunnen worden op literatuur en dus niet gemeten hoeven te worden (parameter fixing, hoofdstuk 5). Door de gevoeligheidsanalyse is dus het patiëntspecifiek maken van het golfvoortplantingsmodel behoorlijk vereenvoudigd; slechts 16 van de 73 inputparameters moeten nauwkeuriger worden gemeten, terwijl voor 47 inputparameters waarden uit de literatuur kunnen worden gekozen. In hoofdstuk 6 is de fysische beschrijving van het golfvoortplantingsmodel gevalideerd met behulp van een experimentele opstelling, waarin de veranderingen in bloeddruk en bloedstroom als gevolg van de aanleg van een AVF werden nagebootst. Hiermee is aangetoond dat het golfvoortplantingsmodel in staat is om de fysische verschijnselen na aanleg van een AVF te beschrijven. Echter, de gesimuleerde bloeddruk- en bloedstroomcurve zijn minder gedempt dan de gemeten bloeddruk- en bloedstroomcurve. Dit is hoogstwaarschijnlijk een gevolg van het niet mee modelleren van de viscoelastische eigenschappen van de experimentele opstelling. Het inzicht verkregen met de gevoeligheidsanalyse en de validatie experimenten, zijn gebruikt in hoofdstuk 7 om de klinische toepasbaarheid van het golfvoortplantingsmodel te onderzoeken. Het model is gebruikt voor 25 patiënten, die lijden aan terminale nierinsufficiëntie, en de simulatieresultaten zijn vergeleken met de klinische uitkomst. Voor $70 \%$ van de patiënten komt de gesimuleerde bloedstroom in de arm overeen met de bloedstroom gemeten 1 week na de operatie. Voor $76 \%$ van de patiënten werd met het golfvoortplantingsmodel dezelfde AVF locatie (boven- of onderarm) gekozen als door een zeer ervaren vaatchirurg. Het ontwikkelde model zou dus een 
extra hulpmiddel kunnen zijn bij de preoperatieve planning van een AVF aanleg. De exacte toegevoegde waarde van het model zal in toekomstig onderzoek aangetoond moeten worden. 


\section{Dankwoord}


Met het afronden van mijn proefschrift bereik ik een nieuwe mijlpaal in mijn leven, die hopelijk na mijn verdediging zal leiden tot de graad van doctor (dr.). Dit vervult mijn (groot)ouders en mijzelf met trots. Helaas kan mijn opa Jelle, die nog wel bij mijn afstuderen was en waar ik een speciale band mee had, dit niet meer meemaken. Poe, ik mis onder andere de discussies over tectonische platen en Ajax en wil je bedanken voor alles! Ook ontbreekt een van mijn beste vrienden en misschien wel de beste, Koen Buijs (24 jaar, 19 augustus 2003). Zijn plotselinge dood heeft diepe indruk op mij gemaakt en mij ontzettend veel verdriet gedaan. Koen, ik vergeet ons laatste gesprek kort voor jouw fatale ongeluk nooit meer! Ook wil ik je bedanken voor de fantastische tijd die wij samen met de rest van de vriendengroep gehad hebben tijdens onze vakanties, kroegentochten, voetbal, oud- en nieuwfeesten etc. In dit kader: prof.dr.ir. Fons Sauren ook erg bedankt voor de goede gesprekken die wij in die periode gehad hebben en die hebben bijgedragen aan de verwerking hiervan.

Verder wil ik mijn eerste promotor Frans bedanken, de fysicus! Frans, ik zeg bewust de fysicus, enerzijds omdat jij mij geleerd hebt dat veel technische problemen (bv. numerieke problemen) vaak veroorzaakt worden door een incomplete beschrijving van de fysica. Maar vooral omdat jij voor mij schijnbaar onoplosbare problemen vaak met logisch nadenken en gebruikmakend van fysische wetten tot een abc-tje kon reduceren. Deze aanpak spreekt mij ontzettend aan en ik heb hier dan ook ontzettend veel van geleerd maar ook bewondering voor! Ook bedankt voor de relaxte manier waarop jij je studenten helpt en benadert. Jouw toegankelijkheid en behulpzaamheid, vind ik persoonlijk heel prettig werken. Ik ben dan ook heel blij dat we deze samenwerking de komende tijd gaan voortzetten. Frans, bedankt!

Mariëlle, de coach, bedankt dat je mijn directe begeleider wilde zijn zowel tijdens mijn promotietijd als tijdens mijn afstuderen. Dit heeft geleid tot de totstandkoming van dit proefschrift maar zeker ook tot mijn persoonlijke ontwikkeling. Talloze keren heb jij conceptversies van artikelen, abstracts en dit proefschrift kritisch gereviseerd en met name gereduceerd. Maar met name heb je mij geleerd op een opbouwend kritische manier naar onderzoeksresultaten te kijken en resultaten in een breder perspectief te plaatsen. Dit is mijn inziens cruciaal voor een onderzoeker en als voetbaltrainer denk ik dat het geven van opbouwende kritiek de manier is om mensen verder te helpen. Ik ben je echter het meest dankbaar voor het feit dat ik altijd bij jou terecht kon als ik vastliep met mijn onderzoek, ik vragen had of als ik een politiekere formulering nodig had voor het presenteren van mijn werk. Mariëlle, bedankt en heel veel geluk met je gezin in Atlanta!

Mijn tweede co-promotor, Jan, de vaatchirurg, bedankt voor het feit dat jij mij de mogelijkheid hebt gegeven om als ingenieur onderzoek te doen in een klinische omgeving 
en dat wij gebruik mochten maken van jouw klinische expertise. Je hebt mij toegelaten tot operaties, andere medische afdelingen zoals het vaatlab en de dialyseafdeling maar ook tot patiëntbesprekingen. Dit heeft mijn kijk op de klinische praktijk verbreed en dit waren voor mij unieke ervaringen die voor een ingenieur die met artsen wil samen werken onmisbaar is. Een ziekenhuisomgeving is namelijk niet te vergelijken met de omgeving die ik gewend was op de technische universiteit. Jan, bedankt!

Mijn 2e promotor, dr. Tammo Delhaas, met jou heb ik slechts een korte periode samengewerkt nadat jij werd aangesteld als het nieuwe hoofd van de vakgroep Biomedische Technologie en ik wil jou bedanken voor het feit dat je vanaf het eerste moment van de verhuizing van de BMT-AzM groep naar de nieuwe afdeling in de universiteit, de voor mij benodigde faciliteiten fantastisch op orde had. Knap dat de verhuizing zo soepel verliep. Ook wil ik je natuurlijk bedanken dat je bereid bent om mijn 2e promotor te zijn. Tammo, bedankt!

Naast het promotieteam, wil ik ook graag de leden van de beoordelingscommissie bedanken. Prof.dr. Schurink bedankt dat u voorzitter van de beoordelingscommissie wilde zijn en dat u bereid was om mijn toch redelijk technisch georienteerde proefschrift kritisch te beoordelen. Prof.dr. Leunissen, prof.dr.ir. Cottaar, prof.dr.ir. Hoeks en prof.dr.ir. Verdonck ook jullie wil ik hier bedanken voor het doornemen van mijn proefschrift. Prof. Hoeks wil ik bovendien bedanken voor de interessante onderwerpen die tijdens de lunch in Maastricht besproken werden en prof. Cottaar voor soortgelijke discussies tijdens de lunch in Eindhoven bij de School of Medical Physics and Engineering (SMPE/e). Prof. Verdonck wil ik nog specifiek bedanken voor de enorme gastvrijheid waarmee ik ontvangen werd als ik een bezoek bracht aan zijn onderzoeksgroep aan de universiteit van Gent. Leden van de beoordelingscommissie, bedankt!

Mijn hele promotieonderzoek is initieel gefinancierd door het profileringsfonds van het azM. Bij het schrijven van de aanvraag heb ik naast Frans, Marielle en Jan ook veel hulp gehad van dr. Nils Planken. Nils, ik wil je dan ook bedanken voor het conceptueel meedenken tijdens de opzet van het onderzoek en voor jouw inbreng, die breder was dan een louter klinische inbreng. Nils, bedankt!

In de loop van het eerste jaar van mijn promotieonderzoek is het onderzoek een onderdeel geworden van een groot Europees project, i.e. het ARCH-project. Ik heb met veel plezier meegewerkt aan dit project and therefore I would like to thank all participants of this project for their contribution, the discussion and the hospitality during visits at their sites. Because it will be very time-consuming to mention all participants individually, I will thank the whole consortium at once. All ARCH-participants, thank you! Specifiek wil ik wel Aron bedanken voor het verzamelen van de klinische data en Koen voor de samen- 
werking tijdens de experimentele validatie van het model. Ook Wilco en Maarten wil ik bedanken die ik als zeer waardevolle collega's binnen het ARCH-project beschouw maar daarnaast ook als collega's BMT-ers. Mensen, bedankt!

Voor de gevoeligheidsanalyse ben ik dank verschuldigd aan Henk Obbink, Ronald van Driel, Wim van der Linden, Cees de Jonge en Marcia Inda die het mogelijk maakte om drie maanden bij Philips te werken. Wim, Marcia and Cees, you were the persons I worked with directly. The critical views of the mathematicians Wim and Marcia, and the practical skills of Cees significantly contributed to this thesis and therefore I would gratefully acknowledge you for this. All Philips people, thanks!

Parallel aan mijn promotieonderzoek liep de traineeship tot qualified medical engineer. Van deze opleiding wil ik specifiek prof.dr. Herman Beijerinck, dr.ir. Ivonne Lammerts, Ineke Fondse en Karlijn Hillekens bedanken. Herman, dankzij jouw kritische kijk en je ervaring in het opleiden van professionals, heb ik me ontwikkeld tot een healthcare professional die in staat is om te werken in een medisch team en begrijpt dat in een klinische praktijk een ietwat pragmatischere aanpak tot betere resultaten kan leiden. Ivonne, jou wil ik bedanken voor het feit dat je mij begeleid hebt tijdens het hele proces en voor je hulp bij het controleren en opzetten van mijn opleidingsplan. Ineke en Karlijn wil ik bedanken omdat zij als office-managers onmisbare schakels zijn binnen de opleiding maar vooral ook omdat ze altijd voor ons klaar stonden als er iets geregeld moest worden of als we even over iets anders wilden praten dan medische technologie. Mensen van de SMPE/e, bedankt!

Natuurlijk was ik er nooit in geslaagd een proefschrift te schrijven als de werksfeer niet goed was. Ik wil dan ook alle mensen van de azM-BMT groep, de nieuwe BMT groep in Maastricht en de mensen bij de SMPE/e bedanken voor de fijne werkomgeving die jullie gecreëerd hebben. Van de collega's die ik nog niet genoemd hebben wil ik specifiek nog Esther, Carole, Maurice, Els, Lambert, Erik, Ellen en Ralf bedanken voor de gezelligheid maar ook de wetenschappelijke discussies tijdens de lunch met de azM-BMT groep. Van de nieuwe BMT-groep wil ik specifiek nog Nico bedanken voor de leuke gesprekken die wij voerden in de trein van Eindhoven naar Maastricht, maar ook Claire die mij hielp bij het invullen van alle papieren die nodig waren om te kunnen promoveren. Bij de SMPE/e wil ik mijn kamergenoot Beatrijs nog bedanken voor de discussies over ons werk maar ook over talloze allerdaagse beslommeringen. Allen, bedankt!

Aangezien ik tijdens mijn promotieonderzoek met talloze mensen heb samengewerkt en om niet iemand per ongeluk te vergeten, wil ik hier zeggen dat ik ieders inbreng ontzettend waardeer. Bedankt!

Naast het werk is mijn grootste passie natuurlijk voetbaltraining geven. Omdat ik er echt 
niet aan ga beginnen om al mijn collega's en spelers waar ik mee samengewerkt heb individueel te bedanken zal ik hen gezamenlijk bedanken voor de fijne samenwerking en de ontspanning die ik krijg door het voetballen. Collega's, spelers en oud-spelers bedankt voor de kampioenschappen, de periodekampioenschappen, de succesvolle samenwerking en de steun!

Verder wil ik mijn familie en vrienden bedanken. Zij waren en zijn er voor mij op momenten dat het tegenzit. Opa en oma Huberts, Poe, Omi, bedankt voor alles! Ook mijn ouders kan ik natuurlijk niet vergeten hier. Pap en Mam, door mij vaker Michael en Sonja genoemd, bedankt voor alles! En Sanne, bedankt dat je mijn zus bent, een betere kan ik me niet wensen. Bedankt ook, dat je paranimf wil zijn. Ook mijn jongste neefje, of moet ik zeggen halfbroertje, Renier, bedankt voor alles en voor het feit dat je ook paranimf wil zijn! Ome Uco, bedankt voor het ontwerpen van de omslag van mijn boekje! Tenslotte wil ik mijn vrienden bedanken die altijd voor me klaar stonden en nog steeds staan. Zonder jullie was het naast mijn werk een stuk saaier geweest. Bedankt, dat jullie het ook konden opbrengen om soms naar het gezeur van de Sonse Sheldon te luisteren. 


\section{Curriculum vitae}




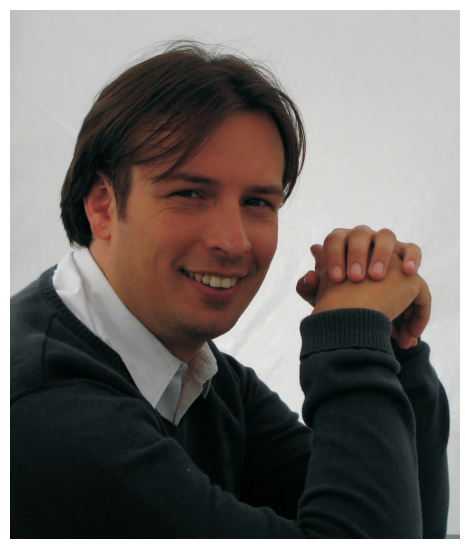

Wouter Huberts was born on the $1^{\text {st }}$ of November, 1979, in Son en Breugel (The Netherlands). After primary school, Wouter went to Gymnasium Beekvliet in Sint-Michielsgestel and succesfully graduated in 1998. Wouter was interested in both physics and medicine but decided to study physics at Eindhoven University of Technology. Although Wouter did like all physics courses, he did not like the experimental part of the study. After one year of physics, Wouter discovered that he preferred to apply physics and engineering to (bio)medical problems. As a logical result, Wouter moved to the study Biomedical Engineering at the same university. After the bachelor phase, Wouter attended the master tract 'Medical Engineering' for which the training programme was mainly organized in Maastricht University Medical Center (The Netherlands). During this master programme Wouter discovered the large differences between a university of technology and a hospital with respect to problem solving, way of thinking, measurements accuracies and hierarchy. Moreover, he gained additional skills mandatory to function within a multidisciplinary medical team. This resulted in a master thesis about the same topic as this $\mathrm{PhD}$ thesis. Thereafter, Wouter further developed to a healthcare professional by attending the post academical programme to Qualified Medical Engineer (QME) at the School of Medical Physics and Engineering, Eindhoven University of Technology. During this programme, Wouter did a three month internship at Philips Research Laboratories Eindhoven (The Netherlands). This two-years in-service programme to QME was successfully completed at Maastricht University Medical Center under supervision of dr. J.H.M. Tordoir (vascular surgeon). This was combined with a four-years $\mathrm{PhD}$ research programme, from which the result is this $\mathrm{PhD}$ thesis.

His major research interests are the development of numerical modeling tools and their application in patient-specific situations, aiming to support decision-making in clinics. Currently, Wouter is appointed as Postdoc at Eindhoven University of Technology, Department of Biomedical Engineering in the Cardiovascular Biomechanics group of Prof.Dr.Ir. F.N. van de Vosse. The research project Wouter is involved now, is closely related to his research interests since Wouter is aiming to develop uncertainty and sensitivity analysis techniques to determine the uncertainty envelope in patient-specific computational models that are developed for cardiovascular, orthopaedic, and disease progression applications and will be used for clinical diagnosis and/or surgery planning. 


\section{Scientific output}




\section{Peer-reviewed journal articles}

W. Huberts, E.M.H. Bosboom and F.N. van de Vosse, A lumped model for blood flow and pressure in the systemic arteries based on an approximate velocity profile function, Math. Biosc. Eng., 6(1), 27-40, (2009)

W. Huberts, A.S. Bode, W. Kroon, R.N. Planken, J.H.M. Tordoir, F.N. van de Vosse, E.M.H Bosboom, A pulse wave propagation model to support decision-making in vascular access planning in the clinic, Med. Eng. Phys., Online available, doi: 10.1016/j.medengphy.2011.07.015, (2011)

W. Huberts, C. de Jonge, W.P.M. van der Linden, M.A. Inda, J.H.M. Tordoir, F.N. van de Vosse, E.M.H. Bosboom, A sensitivity analysis to improve the measurement protocol to assess parameters for a personalized pulse wave propagation model: application to AVF surgery, Submitted, (2011)

W. Huberts, C. de Jonge, W.P.M. van der Linden, M.A. Inda, J.H.M. Tordoir, F.N. van de Vosse, E.M.H. Bosboom, A sensitivity analysis to reduce the number of input parameters in a personalized pulse wave propagation model for AVF surgery, Submitted, (2011)

W. Huberts, K. Van Canneyt, S. Eloot, P. Segers, P. Verdonck, J.H.M. Tordoir, F.N.van de Vosse, E.M.H. Bosboom, Experimental validation of a pulse wave propagation model to predict vascular access surgery, Submitted, (2011)

W. Huberts, A.S. Bode, E.M.H. Bosboom, W. Kroon, W.P.M. van der Linden, R.N. Planken, F.N. van de Vosse, J.H.M. Tordoir, Patient-specific computational modeling of vascular access creation for supporting clinical decision-making, Submitted, (2011)

W. Kroon, W. Huberts, E.M.H. Bosboom, F.N.van de Vosse, A numerical method of reduced complexity for simulating vascular hemodynamics using coupled $O D$ lumped and ID wave propagation models, Comp. Math. Meth. Med., In press, (2012)

A.S. Bode, A. Caroli, W. Huberts, R.N. Planken, L. Antiga, E.M.H. Bosboom, A. Remuzzi, J.H.M. Tordoir, Clinical Study Protocol For The ARCH PROJECT - Computational modeling for improvement of outcome after vascular access creation, J. Vasc. Access, Online available, doi: 10.5301/JVA.2011.8382, (2011) 
W. Kroon, E.M.H. Bosboom, W. Huberts, J.H.M. Tordoir, F.N. van de Vosse, Computational model for estimating the short- and long-term cardiac response to fistula creation for hemodialysis, Submitted, (2012)

M.A.G. Merkx, A.S. Bode, W. Huberts, J. Oliván Bescós, J.H.M. Tordoir, M. Breeuwer, F.N. van de Vosse, E.M.H. Bosboom, Patient-specific hemodynamic analysis using ContrastEnhanced Magnetic Resonance Angiography, image segmentation techniques and computational simulations, Submitted, (2012)

\section{Book chapters}

W. Huberts, E.M.H. Bosboom, R.N. Planken, J.H.M. Tordoir, F.N. van de Vosse, Patientspecific computational modeling to improve the clinical outcome of vascular access creation, EVC textbook 2009: Vascular Access, Edizioni Minerva Medica, Book Chapter, ISBN 10: 88-7711-640-4 (2009)

\section{Conference proceedings and abstracts for oral presenta- tions (only first authorship)}

W. Huberts, E.M.H. Bosboom, R.N. Planken, X. Keuter, J.H.M. Todoir, F.N. van de Vosse, Vascular access surgery for hemodialysis: postoperative flow prediction with a lumped-parameter model, 5th World Congress of Biomechanics, Munich, Germany, (2006)

W. Huberts, E.M.H. Bosboom, R.N. Planken, J.H.M. Tordoir, F.N. van de Vosse, Predicting the clinical outcome of arteriovenous fistulas: the feasibility of patient-specific lumped parameter modeling, Vascular Access for Hemodialysis 11th Symposium, Orlando, United States, (2008)

W. Huberts, E.M.H. Bosboom, J.H.M. Tordoir, F.N. van de Vosse, Predicting the initial postoperative flow after AVF creation for hemodialysis: two modeling approaches, 16th Congress of the European Society of Biomechanics, Lucerne, Switzerland, (2008) 
W. Huberts, E.M.H. Bosboom, R.N. Planken, J.H.M. Tordoir, W. Kroon, F.N. van de Vosse, Predicting the initial postoperative flow after AVF creation for hemodialysis; feasibility of patient-specific modelling, 6th International congress on vascular access, Rome, Italy, (2009)

W. Huberts, E.M.H. Bosboom, A.S. Bode, W. Kroon, J.H.M. Tordoir, F.N. van de Vosse, A patient-specific transmission line model to predict the initial postoperative flow after AVF creation for hemodialysis, 3nd International Meeting of French Hypertension Society, congress l'Hypertension Arteriélle, Paris, France,(2009)

W. Huberts, E.M.H. Bosboom, A. Bode, R.N. Planken, J.H.M. Tordoir, F.N. van de Vosse, A computational model to support the decision-making in vascular access planning, 17th Congress of the European Society of Biomechanics, Edinburgh, United Kingdom, cd, (2010)

W. Huberts, E.M.H. Bosboom, A. Bode, R.N. Planken, W. Kroon, J.H.M. Tordoir, F.N. van de Vosse, The potential of a computational model to support vascular access planning, Virtual Physiological Human Network of Excellence (VPH 2010) congress, Bruxelles, Belgium, (2010)

\section{Approved submissions for project funding}

W. Huberts, E.M.H. Bosboom, R.N. Planken, F.N. van de Vosse, J.H.M. Tordoir, Profileringsfonds, Maastricht University Medical Center, Project name: Vascular remodeling after creation of arteriovenous fistulas for hemodialysis. The predictive value of a patientspecific computer simulation model, (2007) 